\title{
Submanifolds with parallel second fundamental form studied via the Gauß map
}

\author{
Inaugural-Dissertation \\ zur \\ Erlangung des Doktorgrades \\ der Mathematisch-Naturwissenschaftlichen Fakultät \\ der Universität zu Köln \\ vorgelegt von \\ Tillmann Jentsch \\ aus Bergisch Gladbach
}

Hundt Druck GmbH Köln 
Berichterstatter: Prof. Dr. H. Reckziegel

Prof. Dr. G. Thorbergsson

Tag der mündlichen Prüfung: 12.07.2005 


\section{Vorwort}

Zunächst möchte ich meinem Doktorvater, Herrn Professor Dr. H. Reckziegel, herzlich danken. Durch seine wissenschaftliche Anleitung und unsere enge Zusammenarbeit ist die Erstellung dieser Dissertation überhaupt erst möglich geworden. Dabei hat er mir genügend Freiräume gelassen, um eigene Ideen entwickeln zu können; auch dafür möchte ich ihm danken.

Weiterer Dank gilt meinem Mitstreiter Dr. Sebastian Klein, der mir mit vielen nützlichen Tips beratend zur Seite stand.

Schließlich möchte ich meinen Eltern danken, die mich nach Möglichkeit unterstützt haben. 


\begin{abstract}
For an arbitrary $n$-dimensional riemannian manifold $N$ and an integer $m \in\{1, \ldots, n-1\}$ a covariant derivative $\hat{\nabla}$ on the Graßmann bundle $\hat{N}:=\mathrm{G}_{m}(T N)$ is introduced which has the property that an $m$-dimensional submanifold $M \subset N$ has parallel second fundamental form if and only if its Gauß map $M \rightarrow \hat{N}$ is affine. (For $N=\mathbb{R}^{n}$ this result was already obtained by J. VILMS in 1972.) By means of this relation a generalization of É. CARTAN's theorem on the total geodesy of a geodesic umbrella can be derived: Suppose, initial data $(p, W, b)$ prescribing a tangent space $W \in \mathrm{G}_{m}\left(T_{p} N\right)$ and a second fundamental form $b$ at $p \in N$ are given; for these data an $m$-dimensional "umbrella" $M=M_{U}(p, W, b) \subset N$ gets constructed, the rays of which are helical arcs of $N$; moreover tensorial conditions (not involving $\hat{\nabla}$ ) are presented, which guarantee that the umbrella $M$ has parallel second fundamental form. These conditions are as well necessary, and locally every submanifold with parallel second fundamental form can be obtained in this way.
\end{abstract}




\section{Contents}

1 Introduction 1

2 Helical arcs and helical umbrellas $\quad 5$

3 The main result $\quad 21$

4 Semiparallelity of the second fundamental form and the euclidean Jordan triple property $\quad 24$

$5 \quad$ Geometry over the Graßmann bundle of $N \quad 27$

6 Geometry of the manifold $\hat{N} \quad 33$

7 Distinguished $m$-planes of $T \hat{N} \quad 47$

8 Proof of Theorem 1

9 A globalization of Theorem 1

10 Geometry of strips $\quad 61$

11 The generalization to indefinite metrics $\quad 63$

12 II-parallel isometric immersions adapted to special riemannian geometries

$\begin{array}{ll}\text { References } & 81\end{array}$

Zusammenfassung in deutscher Sprache $\quad 83$ 


\section{Introduction}

In a riemannian manifold $N$ the most "straight" submanifolds are the totally geodesic ones. In general, totally geodesic submanifolds of dimensions $\geq 2$ and $<\operatorname{dim} N$ occur very scarcely; however É. CARTAN had proved already the following criterion:

Theorem (É. Cartan). If a point $p \in N$, a linear subspace $V \varsubsetneqq T_{p} N$ of dimension $\geq 1$ and some star shaped neighborhood $U$ of 0 in $V$ are given such that the exponential map $\exp _{p}^{N}$ of $N$ at $p$ is defined on $U$ and is there an injective immersion into $N$, then the "geodesic umbrella" $M_{U}(p, V):=\exp _{p}^{N}(U)$ is a totally geodesic submanifold of $N$ if and only if for every $u \in U$ the parallel translate $V_{u}$ of $V$ along the geodesic arc $c_{u}:[0,1] \rightarrow N, t \mapsto \exp _{p}^{N}(t u)$,

$$
\left.\left.V_{u}:=\underset{0}{\left(\| c_{u}\right.}\right)^{N}(V) \subset T_{\exp _{p}^{N}(u)} N \quad \text { (see below }(1)\right),
$$

is curvature invariant ("integrability condition"), that means $R\left(v, v^{\prime}\right) v^{\prime \prime} \in V_{u}$ for all $v, v^{\prime}, v^{\prime \prime} \in$ $V_{u}$. Notice, $p \in M_{U}(p, V)$ and $T_{p}\left(M_{U}(p, V)\right)=V$.

A famous special case of this theorem is the well known relation between Lie triple systems and totally geodesic submanifolds in the theory of symmetric spaces. - In this thesis the analogous problem for submanifolds with parallel second fundamental form, in the literature sometimes called parallel submanifolds, is solved; thereby the terminology II-parallel submanifold is more likely to be used, since it refers explicitly to the second fundamental form. A survey on II-parallel submanifolds can be found in [L].

In real space forms the II-parallel submanifolds were classified by Ferus, TAKEuchi, BaCKES and Reckziegel [F, Ta, BR]; in particular, it was shown that they are always open parts of complete ones and that the latter are exactly the (extrinsic) symmetric submanifolds in the sense of FERUS $[F]$. In symmetric spaces the symmetric submanifolds were classified by NAITOH, Takeuchi, Berndt, Eschenburg, Tsukada and others (see [NT, B-T]), after Naitoh had already shown in [Na1] that these symmetric submanifolds always are II-parallel.

If $M \subset N$ is a II-parallel submanifold, then every unit speed geodesic of $M$ is a helical arc (a concept including geodesics, circles and genuine helices) in the ambient space $N$ (first proved by Strübing $[\mathrm{S}])$. Therefore the geodesic umbrella of CARTAN's theorem has to be replaced in case of this problem by a helical umbrella $M_{U}(p, W, b)$; here $p$ is an arbitrary point of $N, W \subset T_{p} N$ is the prescribed tangent space for the II-parallel submanifold $M$ to be looked for, $b: W \times W \rightarrow W^{\perp}$ is the prescribed second fundamental form for $M$ at $p$ and $U$ a sufficiently small starshaped neighborhood of 0 in $W$. In fact, this helical umbrella satisfies the initial data $(p, W, b)$, and if $W$ is curvature invariant, then the covariant derivative of its second fundamental form vanishes at $p$ (see Proposition 8).

In order to give some insight in the main result (Theorem 1) the space $\operatorname{End}_{-}\left(T_{p} N\right)$ of skewadjoint endomorphisms of $T_{p} N$ and its linear subspace

$$
\mathbb{M}_{W}:=\left\{A \in \operatorname{End}_{-}\left(T_{p} N\right) \mid A(W) \subset W^{\perp} \text { and } A\left(W^{\perp}\right) \subset W\right\}
$$

are introduced. Definition 1 associates with $b$ a linear map $A^{b}: W \rightarrow \mathbb{M}_{W}, u \mapsto A_{u}^{b}$; then for every $u \in W$ the helical arc $c_{u}$, the analogue of the geodesic of Cartan's theorem, will be defined by means of the 1-parameter subgroup $t \mapsto \operatorname{Exp}\left(t A_{u}^{b}\right) \in \mathrm{SO}\left(T_{p} N\right)$, which comprises 
most effectively the helical character of $c_{u}$ (see Definition 3). The helical umbrella now is defined by

$$
M_{U}(p, W, b):=\left\{c_{u}(1) \mid u \in U\right\} .
$$

It should be mentioned that, if there exists a II-parallel submanifold $M$ with the initial data $(p, W, b)$, then it always contains such a sufficiently small helical umbrella $M_{U}(p, W, b)$ as an open subset (see Proposition 5(c) and Corollary 4).

Lemma 4 shows how the second fundamental form of a submanifold $M \subset N$ determines the "twisting" of the tangent spaces of $M$ in $T N$ along a curve $c: J \rightarrow M$ with $c(0)=p$ : Namely, by parallel transport of the second fundamental form of $M$ along $c$ back to $T_{p} N$ (and using the above mentioned map $b \mapsto A^{b}$ ) one obtains a function $A: J \rightarrow \operatorname{End}_{-}\left(T_{p} N\right)$, and if $g: J \rightarrow \mathrm{SO}\left(T_{p} N\right)$ is the solution of the the differential equation $g^{\prime}(t)=g(t) \circ A(t)$ with $g(0)=\mathrm{id}_{T_{p} N}$, then the linear isometry

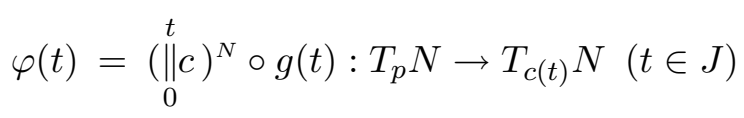

maps $T_{p} M$ (resp. $\perp_{p} M$ ) by parallel transport within $M$ (resp. within the normal bundle $\perp M$ ) onto $T_{c(t)} M$ (resp. onto $\perp_{c(t)} M$ ). In case that $c=c_{u}$ is a geodesic of a II-parallel submanifold with $\dot{c}_{u}(0)=u$ this $M$-split-parallel displacement of $T_{p} N$ along $c_{u}$ turns out to be the isometry

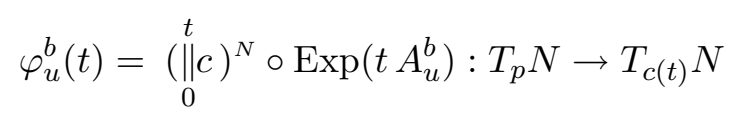

for every $t \in J$ (see Proposition 5(c)). As a consequence of this, as an substitute for the parallel translation along $c_{u}$ in Cartan's above mentioned theorem these isometries $\varphi_{u}^{b}(t)$ must serve; hence $V_{u}$ has to be replaced by $\xi_{u}(t):=\varphi_{u}^{b}(t)(W)$; and in addition a bilinear map $h_{u}(t): \xi_{u}(t) \times \xi_{u}(t) \rightarrow \xi_{u}(t)^{\perp}$ has to be constructed by transporting $b$ to $T_{c_{u}(t)} N$ via $\varphi_{u}^{b}(t)$. Now Theorem 1 states:

The helical umbrella $M_{U}(p, W, b)$ is II-parallel if and only if for all unit vectors $u \in W$ the subspaces $\xi_{u}(t)$ are curvature invariant and the bilinear maps $h_{u}(t)$ are semiparallel (see Definition 1(b)).

In [PR2] PAWEL and RECKZIEGel already solved the problem for spherical submanifolds (constituting a subclass of the set of all II-parallel submanifolds) by constructing circular umbrellas (see also Example 7).

From Corollary 5 one sees that in a space $N$ of constant curvature there exists a II-parallel submanifold $M$ with the initial data $(p, W, b)$ if and only if $b$ is $R_{p}^{N}$-semiparallel. There is known a quite different sufficient and necessary tensorial condition for the existence of II-parallel submanifolds in standard spaces discovered by D. FeRUs in [F] for $N=\mathbb{R}^{n}$ and later extended to the other spaces of constant curvature in [BR] (see Section 2 there), namely that a certain bilinear map $L: W \times W \rightarrow \operatorname{End}(W)$ associated with the triple $(p, W, b)$ is a euclidean Jordan triple system. In Section 4 Ferus' calculations will be presented in such a way that one is able to prove (by purely tensorial calculations) that in case of a constant curvature $N$ the conditions of Ferus and ours are equivalent (see Theorem 3).

For the proof of Theorem 1 in Section 5 a framework which concerns the differential geometry (up to second order) for arbitrary $m$-dimensional submanifolds $M \subset N$ (without reffering to 
a special submanifold $M$ ) is developed. As the splittings $T_{p} N=T_{p} M \oplus \perp_{p} M$ for $p \in M$ are crucial, the Graßmann bundle $\tau: \hat{N} \rightarrow N$ with fibres $\hat{N}_{p}:=\mathrm{G}_{m}\left(T_{p} N\right)$ and the following bundles over $\hat{N}$ will be considered: the pull back vector bundle $\mathbb{E}:=\hat{N} \times{ }_{N} T N \rightarrow \hat{N}$ (with fibre $T_{\tau(W)} N$ over $\left.W \in \hat{N}\right)$, the canonical splitting $\mathbb{E}=\pi \oplus \Perp$ which at a "point" $W \in \hat{N}_{p}$ is given by $T_{p} N=W \oplus W^{\perp}$, the principal bundle $\mathbb{P}$ of orthonormal frames of $\mathbb{E}$ adapted to this splitting, the vector bundle $\mathbb{B}$ of "potential" second fundamental forms given by $\mathbb{B}_{W}:=\mathrm{L}^{2}\left(W, W^{\perp}\right)$ and the vector bundle $\mathbb{M}$ whose fibres $\mathbb{M}_{W}$ had been described above. The bundle $\mathbb{P}$ is canonically equipped with a connection $\mathcal{H}^{\mathbb{P}}$ which induces distinguished covariant derivatives of the associated vector bundles $\mathbb{E}, \mathbb{B}$ and $\mathbb{M}$. In particular the parallel displacement in $\mathbb{E}$ will be a universal split-parallel displacement, it describes the $M$-split-parallel displacement for arbitrary $m$-dimensional submanifolds. Moreover, if the second fundamental form of an $m$-dimensional submanifold $M$ is considered as a section in $\mathbb{B}$ along its Gauß map, then its covariant derivative (just mentioned) coincides with the one usually used in submanifold geometry. Key object in this treatment is a certain differential 1-form $\hat{\nu}$ on $\hat{N}$ with values in the bundle $\mathbb{M}$, which "contains" the second fundamental form of arbitrary $m$-dimensional submanifolds of $N$; using it one can formulate universal Gauß and Weingarten equations and a universal formula combining the curvature equations of Gauß, Codazzi and Ricci (see (75), (78) and Example 9).

On the other hand, for every $q \in N$ and $W \in \hat{N}_{q}$ the restriction $\hat{\nu} \mid T_{W} \hat{N}_{q}$ is the well known isomorphism between the tangent space of the symmetric space $\mathrm{G}_{m}\left(T_{q} N\right)$ at the "point" $W$ and the subspace $\mathbb{M}_{W}$ of the Lie algebra $\operatorname{End}_{-}\left(T_{q} N\right)$ of the transformation group $\mathrm{O}\left(T_{q} N\right)$ (see Remark 7 and Theorem 4). This fact enables one to construct a linear connection $\hat{\nabla}$ (with torsion) on the manifold $\hat{N}$ (see Section 6), which - concerning this problem - seems to be adapted to the geometry of the Graßmann bundle in the best possible way. In fact, for every $m$-dimensional submanifold $M$ a relation between the covariant derivative of its second fundamental form and the second fundamental form of the Gauß map $\xi^{M}$ of $M$ (see Theorem 6) will be derived; as a Corollary of this one gets the important geometric insight:

$M$ has a parallel second fundamental form if and only if $\xi^{M}$ is an affine map into $(\hat{N}, \hat{\nabla})$.

The theorem mentioned just now and its corollary generalize VILMS' well known results from [V2] concerning submanifolds in euclidean space.

Further objects, which are crucial for the investigations done in this thesis, are distinguished $m$-dimensional subspaces $\mathcal{T}_{b} \subset T_{W} \hat{N}$ associated with the bilinear maps $b \in \mathbb{B}_{W}$ (see Section 7). For $m$-dimensional submanifolds they play a similar role as the spaces of acceleration vectors $\in T(T N)$ for curves of $N$, which also do not fill the entire second order tangent bundle of $N$ (see Theorem 7). In fact, such "higher ordered tangent spaces" of submanifolds were already investigated by AmBrose in $[\mathrm{A}]$; the relation between his investigation and the subspaces $\mathcal{T}_{b}$ is described in Theorem 7 and Proposition 16. As a byproduct I obtain a new criterion for the involutivity of a distribution (see Corollary 11).

Now one can conclude: The construction of a submanifold $M \subset N$ with parallel second fundamental form for given initial data $(p, W, b)$ is equivalent to the construction of an affine (=autoparallel) submanifold in $(\hat{N}, \hat{\nabla})$ for the initial data $\left(W, \mathcal{T}_{b}\right)$. The only local candidates for such affine submanifolds in $\hat{N}$ are the geodesic umbrellas described in Cartan's theorem. As was shown in [PR1] this theorem remains valid in every "affine" manifold $(\hat{N}, \hat{\nabla})$, if the condition " $V_{u}$ is curvature invariant" is replaced by " $V_{u}$ is curvature and torsion invariant". In Proposition 14 these "integrability conditions" are translated into the "integrability conditions" 
for the parallelity of the second fundamental form of the helical umbrella $M_{U}(p, W, b)$. After that the proof of the main result is finished quickly (see Section 8).

In Section 9 a global version of Theorem 1 is proved. In particular, one obtains that in a symmetric space $N$ a II-parallel isometric immersion from a complete riemannian manifold into $N$ exists for prescribed initial data $(p, W, b)$, if an arbitrarily small helical umbrella $M_{U}(p, W, b)$ is II-parallel. This local condition can be checked by testing only a finite number of tensorial equations at the point $p$ (see Theorem 2).

For the sake of simplicity I have supposed in this paper that the riemannian metric of $N$ is positive definite. In the case of a pseudoriemannian manifold $N$ of index $k \in\{1, \ldots, \operatorname{dim} N-1\}$ the prescribed tangent space $W \in \mathrm{G}_{m}\left(T_{p} N\right)$ in Theorem 1 must be supposed to be nondegenerate (with resprect to the metric of $N$ ); if this pseudoeuclidean vector space has index $\ell \in\{0, \ldots, \min \{m, k\}\}$, the Graßmann bundle $\tau: \hat{N} \rightarrow N$ has to be restricted to the set of the subspaces of this kind. In Section 11 this fact and the necessary modifications of the investigation are described in detail. Here I will only mention one fact. In a complete riemannian manifold the maximal helical arcs are defined on the whole real line $\mathbb{R}$. But this is not true in the pseudoriemannian case, because a geodesically complete pseudoriemannian manifold in general is not totally complete; in Proposition 3 an essential exception is described, namely pseudoriemannian symmetric spaces (more generally reductive homogeneous spaces with the canonical connection); although this fact is known, there is given a proof, because I could not find one in textbooks.

So far the content of the thesis was described with regard to Theorem 1, the analogue of CARTAN's theorem cited at the beginning. In fact, the purpose of the thesis is also to give more insight into the geometry of II-parallel submanifolds. For instance, the description of helical arcs in Definition 3 and of the twisting of the tangent space of a II-parallel submanifold along a geodesic in Proposition 5 (already presented in [JR]) seem to be new. In fact, this twisting of tangent planes occurs also in a slightly more general situation (see Proposition 6 and Corollary 3). Furthermore, Corollary 4 shows that the well known rigidity of II-parallel submanifolds can be extended to submanifolds, for which some $k$-th covariant derivative of the second fundamental form vanishes identically. Also Corollary 2 should be mentioned, which says that a II-parallel submanifold of a locally symmetric space again is locally symmetric, a result which is stated in [Na1] without proof. As the second fundamental form $h^{M}$ of a submanifold $M$ is the primary object of the thesis, I will here also mention Proposition 1, which gives an interpretation of $h^{M}$ by means of the infinitesimal movement of the tangent spaces of $M$ along curves. In Section 6 it is shown that this fact can be derived also via the "universal second fundamental form" $\hat{\nu}$ from a very general observation concerning associated fibre bundles (see Lemma 6).

As well I could have declared the generalization of VILMS' result (Theorem 6 and Corollary 10) as the main topic of this thesis. In regard to this it was of interest to compare the linear connection $\hat{\nabla}$ with the Levi-Civita connection $\nabla^{L C}$ of the canonical riemannian metric of the Graßmann bundle; the main properties of $\nabla^{L C}$ are described in Proposition 12; the comparision can be found in Section 6 (Theorem 5, Corollary 8 and 9).

The last Section deals with a riemannian manifold $N$ which is equipped with an additional "parallel" geometric structure (see Definition 13). Then it is asked for II-parallel isometric immersions, which in some sense are compatible with this structure. Starting with a distinguished subspace $W_{0} \in \hat{N}$ there is constructed a parallel subbundle $\hat{N}\left(W_{0}\right) \rightarrow N$ of $\tau: \hat{N} \rightarrow N$, 
the fibres of which are symmetric subspaces of the fibres of $\tau$ and the bundle space of which turns out to be an affine submanifold of $(\hat{N}, \hat{\nabla})$ (see assertion (i) of Example 16(c) and Proposition 24). An $m$-dimensional II-parallel isometric immersion $f: M \rightarrow N$, whose Gauß map satisfies $\xi^{f}(M) \subset \hat{N}\left(W_{0}\right)$ are considered as compatible with the given structure of $N$, and the condition $\xi^{f}(M) \subset \hat{N}\left(W_{0}\right)$ is fulfilled, if at one point $p \in M$ the 1-jet of $\xi^{f}$ is in accordance with this fact (see Theorem 12). That this abstract theory makes sense is demonstrated by two examples, namely by complex and Lagrangian II-parallel submanifolds in Kählerian manifolds (see Corollary 14) and by extrinsic symmetric submanifolds in symmetric spaces (see Corollary 15 and Theorem 13).

Lastly some words to Section 10 are in order. In it the theory for II-parallel submanifolds in $N$ is extended to strips along arbitrary differentiable maps $f: M \rightarrow N$. (Strips can be considered as generalizations of immersions, since the Gauß map of an immersion is a strip.) Having done this one recovers easily a theorem describing a condition that the image of a differentiable map is contained in a II-parallel submanifold (Theorem 11); this result includes ERBACHER's theorem on the reduction of codimension for isometric immersions in real space forms; see [R, Theorem 1$]$ and $[\mathrm{E}]$.

\section{$2 \quad$ Helical arcs and helical umbrellas}

In this thesis all manifolds, maps etc. are assumed to be $\mathrm{C}^{\infty}$-differentiable if not otherwise stated. $N$ always denotes a connected riemannian manifold of dimension $n ;\langle\cdot, \cdot\rangle, \nabla^{N}$ and $R^{N}$ denote its riemannian metric, Levi-Civita connection and curvature tensor, respectively. Furthermore, for any curve $c: J \rightarrow N$ and any $t_{1}, t_{2} \in J$ let

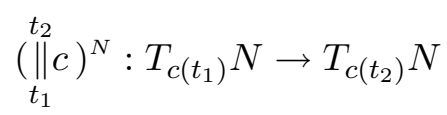

denote the parallel displacement in $N$ along $c$. For all other manifolds the analogous geometric objects will be marked by an appropriate index. If $f: M \rightarrow N$ is an isometric immersion from a further riemannian manifold $M$, its second fundamental form and shape operator are denoted by $h^{f}$ resp. $S^{f}$, its mean curvature vector field by $H^{f}$, its normal bundle by $\perp f$, the canonical connection on $\perp f$ by $\nabla^{\perp f}$ and the curvature tensor of $\nabla^{\perp f}$ by $R^{\perp f}$. The covariant derivative of $h^{f}$ is defined by

$$
\left(\bar{\nabla}_{X} h^{f}\right)(Y, Z)=\nabla_{X}^{\perp f} h^{f}(Y, Z)-h^{f}\left(\nabla^{M} Y, Z\right)-h^{f}\left(Y, \nabla_{X}^{M} Z\right)
$$

for $X, Y, Z \in \Gamma(T M)$. To say that $h^{f}$ is parallel means $\bar{\nabla} h^{f}=0$. For a submanifold $M \subset N$ the normal bundle $\perp M$, second fundamental form $h^{M}$, shape operator $S^{M}$ and mean curvature vector field $H^{M}$ are those of the inclusion map $M \hookrightarrow N$. If the second fundamental form of an isometric immersion or a submanifold is parallel, we will also speak shortly of II-parallel isometric immersions resp. submanifolds. For the fundamentals of submanifold geometry see $[\mathrm{Ch}]$.

For any vector bundle $E \rightarrow N$ and any $\mathrm{C}^{\infty}$-map $f: M \rightarrow N$ the $\mathrm{C}^{\infty}(M)$-module of sections in $E$ along $f$ will be denoted by $\Gamma_{f}(E)$. Finally, by $\partial$ we denote the canonical unit vector field on $\mathbb{R}$; for instance, for a curve $c: J \rightarrow N$ and a vector field $Y \in \Gamma_{c}(T N) \quad \nabla^{N_{\partial}} Y$ is its covariant derivative. ${ }^{1}$

\footnotetext{
${ }^{1}$ For the covariant differentiation of vector fields along maps see $[\mathrm{P}]$ p.36.
} 
Example 1 (Spherical immersions).

(a) Every spherical immersion $f$ is II-parallel, because then $h^{f}(X, Y)=\langle X, Y\rangle H^{f}$ with $\nabla^{\perp f} H^{f}=0$ holds by definition. Moreover, every totally geodesic immersion $f$ is spherical (with $H^{f}=0$ ) and therefore II-parallel.

(b) A unit speed curve $c: J \rightarrow N$ (which of course is an isometric immersion) is II-parallel if and only if it is a circular arc, i.e., if it satisfies the differential equation

$$
\nabla_{\partial}^{N} \nabla_{\partial}^{N} \dot{c}+\left\langle\nabla_{\partial}^{N} \dot{c}, \nabla_{\partial}^{N} \dot{c}\right\rangle \cdot \dot{c}=0
$$

where automatically $\left\langle\nabla_{\partial}^{N} \dot{c}, \nabla_{\partial}^{N} \dot{c}\right\rangle$ is constant, see $\S 1$ in [LN].

As the second fundamental form of a submanifold is of primary interest in this thesis, I will give now a very geometric description using the Graßmann manifold $\mathrm{G}_{m}\left(T_{p} N\right)$ of the $m$-dimensional linear subspaces of the tangent space $T_{p} N$.

Proposition 1 (A Graßmann approach towards the second fundamental form). Let be given an m-dimensional submanifold $M$ of a riemannian manifold $N$ and a point $p \in M$. Then the second fundamental form $h_{p}^{M}$ of $M$ at $p$ can be obtained in the following way: If $c: J \rightarrow M$ is a curve with $c(0)=p$ and $\dot{c}(0)=: u$, define the curve

$$
\begin{aligned}
\xi: J \rightarrow \mathrm{G}_{m}\left(T_{p} N\right), t \mapsto & \stackrel{0}{(\| c)^{N}\left(T_{c(t)} M\right)} \\
& =\text { the parallel displacement of } T_{c(t)} M \text { in } N \text { along } c \text { to } p
\end{aligned}
$$

in the Graßmann manifold $\mathrm{G}_{m}\left(T_{p} N\right)$ and put $W:=T_{p} M$. Then for the parameters $t$ of a sufficiently small neighbourhood $U(0) \subset J$ the linear subspaces $\xi(t) \subset T_{p} N=W \oplus W^{\perp}$ can be interpreted as the graphs of linear maps $\ell(t): W \rightarrow W^{\perp}$ and by ordinary differentiation of the function $\ell: U(0) \rightarrow \mathrm{L}\left(W, W^{\perp}\right)$ one obtains

$$
h_{p}^{M}(u, v)=\ell^{\prime}(0) v \quad \text { for all } \quad v \in T_{p} M .
$$

Proof. First notice that $\xi$ is a differentiable curve in $\mathrm{G}_{m}\left(T_{p} N\right)$ and that the graphs of all linear maps $W \rightarrow W^{\perp}$ constitute a neighbourhood $\mathcal{U}(W)$ of $\xi(0)=W$ in $\mathrm{G}_{m}\left(T_{p} N\right)$; this implies the existence of the neighbourhood $U(0)$.

Now, for every vector $v \in W$ let $Y^{v}$ denote the $\nabla^{M}$-parallel vector field in $M$ along $c$ with $Y^{v}(0)=v$ and define its "backward parallel displacement"

$$
\hat{Y}^{v}: J \rightarrow T_{p} N, t \mapsto \underset{t}{\stackrel{0}{\| c})^{N}} Y^{v}(t)
$$

(see the following Definition 2 and Proposition 2(a)); notice that one has $\left(\nabla_{\partial}^{N} Y^{v}\right)_{t=0}=\left(\hat{Y}^{v}\right)^{\prime}(0)$. Therefore we obtain for $t \in U(0)$

$$
h_{p}^{M}(u, v)=\left(\nabla_{\partial}^{N} Y^{v}-\nabla_{\partial}^{M} Y^{v}\right)_{t=0}=\left(\hat{Y}^{v}\right)^{\prime}(0) .
$$

Using the orthogonal projections

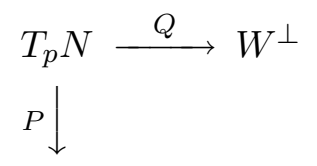

W 
and the fact that $\left\{\hat{Y}^{v}(t) \mid v \in W\right\}=\xi(t)=\{v+\ell(t) v \mid\}$ by our choice of the linear map $\ell(t)$ we get for every $t \in U(0)$ and $v \in W$

$$
\ell(t) P \hat{Y}^{v}(t)=Q \hat{Y}^{v}(t) \text { and therefore } \quad \ell^{\prime}(t) P \hat{Y}^{v}(t)+\ell(t) P\left(\hat{Y}^{v}\right)^{\prime}(t)=Q\left(\hat{Y}^{v}\right)^{\prime}(t) .
$$

Since $\ell(0) \equiv 0$ and $\hat{Y}^{v}(0)=v$ and because of $(3)$, we obtain $\ell^{\prime}(0) v=Q h_{p}^{M}(u, v)=$ $h_{p}^{M}(u, v)$.

In the previous proof we have used the subset $\mathcal{U}(W)$. In the general situation of a euclidean vector space $V$ with $\operatorname{dim} V=: n>m$ and a subspace $W \in \mathrm{G}_{m}(V)$ the analogous set $\mathcal{U}(W) \subset$ $\mathrm{G}_{m}(V)$ is well known to be open and dense in $G_{m}(V)$; furthermore, the map

$$
x: \mathcal{U}(W) \rightarrow \mathrm{L}\left(W, W^{\perp}\right) \quad \text { characterized by } \quad W^{\prime}=\text { graph of } x\left(W^{\prime}\right)
$$

is a $\mathrm{C}^{\infty}$-chart of the manifold $\mathrm{G}_{m}(V)$; obviously this chart is canonical with respect to the subspace $W$. Thus we obtain the canonical isomorphism

$$
d x: T_{W} \mathrm{G}_{m}(V) \rightarrow \mathrm{L}\left(W, W^{\perp}\right) ;
$$

With this terminology we can formulate the result of Proposition 1 by

$$
h_{p}^{M}(u, v)=d x(\dot{\xi}(0)) v \text { for all } v \in T_{p} M .
$$

As is well known, $\mathrm{G}_{m}(V)$ is a symmetric space $\cong \mathrm{O}(V) / H$ with the isotropy group

$$
H:=\{g \in \mathrm{O}(V) \mid g(W)=W\}
$$

(e.g. see $[\mathrm{By}]$, p. 362). The corresponding decomposition of the Lie algebra $\mathfrak{o}(V)=$ End $_{-}(V)$ is $\mathfrak{o}(V)=\mathfrak{h} \oplus \mathfrak{m}$ with the Lie algebra $\mathfrak{h}$ of $H$ and the subspace

$$
\mathfrak{m}:=\mathbb{M}_{W}:=\left\{A \in \operatorname{End}_{-}(V) \mid A(W) \subset W^{\perp} \text { and } A\left(W^{\perp}\right) \subset W\right\} .
$$

The notation $\mathbb{M}_{W}$ is introduced for further convenience. Furthermore,

$$
\Theta_{W}: \mathbb{M}_{W} \rightarrow T_{W} \mathrm{G}_{m}(V),\left.A \mapsto \frac{\mathrm{d}}{\mathrm{d} t}\right|_{t=0}(\operatorname{Exp}(t A)(W))
$$

is the isomorphism always used in the theory of symmetric spaces. In fact, the isomorphisms in (5) and (8) are related in the simplest way to each other, namely by

$$
\forall A \in \mathbb{M}_{W}: d x\left(\Theta_{W}(A)\right)=A \mid W .
$$

The proof of (9) runs in the lines of the proof of Proposition 1 with the following modifications:

$$
\begin{array}{llll}
\text { instead of } \hat{Y}^{v} \text { use } \quad f_{v}: \mathbb{R} \rightarrow V, t \mapsto \operatorname{Exp}(t A)(v) \quad \text { for every } \quad v \in W \quad \text { and } \\
\text { instead of } \xi(t) \text { use } \quad \xi(t):=\operatorname{Exp}(t A)(W)=\left\{f_{v}(t) \mid v \in W\right\} \quad \text { for every } t \in \mathbb{R},
\end{array}
$$

and notice that we have $f_{v}^{\prime}(t)=\operatorname{Exp}(t A)(A v)$ now and therefore $f_{v}^{\prime}(0)=A v \in W^{\perp}$.

Combining (6) and (9) we obtain

Corollary 1. If in the situation of Proposition $1 A \in \mathbb{M}_{W}$ is the endomorphism characterized by $A v=h_{p}^{M}(u, v)$ for all $v \in T_{p} M$, then $\Theta_{W}(A)=\dot{\xi}(0) \quad$ (see also Definition 1(a) and Example 2(a)). 
Before we start with the investigation of II-parallel submanifolds, we have to make some preparations.

Definition 1. Let $V$ be a euclidean vector space, $W \neq\{0\}$ a proper linear subspace of $V$, End- $(V)$ the vector space of skew-adjoint endomorphisms of $V$ and $\mathrm{L}\left(W, W^{\perp}\right)$ resp. $\mathrm{L}^{2}\left(W, W^{\perp}\right)$ the vector spaces of linear resp. bilinear maps $\ell: W \rightarrow W^{\perp}$ resp. $b: W \times W \rightarrow$ $W^{\perp}$.

(a) For every $\ell \in \mathrm{L}\left(W, W^{\perp}\right)$ let $A_{\ell} \in \mathbb{M}_{W}$ be the unique element with $A_{\ell} \mid W=\ell$ (see (7)); notice, $A_{\ell} \mid W^{\perp}=-\ell^{*}$ (where $\ell^{*}: W^{\perp} \rightarrow W$ denotes the adjoint of $\ell$ ) and the map $\mathrm{L}\left(W, W^{\perp}\right) \rightarrow \mathbb{M}_{W}: \ell \mapsto A_{\ell}$ is an isomorphism. For every pair $(b, v) \in \mathrm{L}^{2}\left(W, W^{\perp}\right) \times W$ we put $A_{v}^{b}:=A_{b(v, \cdot)}$.

(b) If $R$ is an algebraic curvature tensor of type (1,3) on $V$ (see [Be]p.46) and $b \in$ $\mathrm{L}^{2}\left(W, W^{\perp}\right)$, then we associate a skew-symmetric bilinear map $R^{b}: W \times W \rightarrow \operatorname{End}_{-}(V)$ with the quadruple $(V, W, b, R)$ by

$$
R^{b}\left(u_{1}, u_{2}\right):=R\left(u_{1}, u_{2}\right)-\left[A_{u_{1}}^{b}, A_{u_{2}}^{b}\right] \quad \text { for all } u_{1}, u_{2} \in W
$$

here $[\cdot, \cdot]$ denotes the commutator in $\operatorname{End}_{-}(V)$. It should be noticed that $\left[A_{u_{1}}^{b}, A_{u_{2}}^{b}\right](W) \subset$ $W$ and $\left[A_{u_{1}}^{b}, A_{u_{2}}^{b}\right]\left(W^{\perp}\right) \subset W^{\perp}$. If in addition, $W$ is $R$-invariant, i.e., $R(W, W)(W) \subset W$, then we have $R^{b}(W, W)(W) \subset W$ and $R^{b}(W, W)\left(W^{\perp}\right) \subset W^{\perp}$ therefore; in this situation we will say that $b$ is $R$-semiparallel, if for all $u_{1}, u_{2}, v_{1}, v_{2} \in W$ we have

$$
R^{b}\left(u_{1}, u_{2}\right)\left(b\left(v_{1}, v_{2}\right)\right)=b\left(R^{b}\left(u_{1}, u_{2}\right) v_{1}, v_{2}\right)+b\left(v_{1}, R^{b}\left(u_{1}, u_{2}\right) v_{2}\right) ;
$$

see also Remark 8.

(c) For every $A \in$ End $_{-}(V)$ and $v \in V$ let $V(A, v)$ denote the smallest $A$-invariant subspace of $V$ containing $v$, and let $\beta_{v}^{A}$ denote the orbit $\mathbb{R} \rightarrow V, t \mapsto \operatorname{Exp}(t A) v$ of the 1parameter subgroup $\mathbb{R} \rightarrow \mathrm{SO}(V), t \mapsto \operatorname{Exp}(t A) ; \operatorname{dim} V(A, v)$ will also be called the rank of the orbit $\beta_{v}^{A}$ (see Lemma 1(b)).

Example 2. (a) Significance of the tensors $A_{u}^{b}$ in submanifold geometry. If $M$ is a submanifold with shape operator $S^{M}$, then for every $p \in M$ and $u \in W:=T_{p} M$ the endomorphism $A_{u}^{b}$ with $b:=h_{p}^{M}$ satisfies

$$
\forall(v, z) \in T_{p} M \times \perp_{p} M: A_{u}^{b}(v)=h^{M}(u, v) \text { and } A_{u}^{b}(z)=-S_{z}^{M} u .
$$

Notice that the endomorphism $A$ in Corollary 1 concides with $A_{u}^{b}$. Furthermore, if we assign the tensor $R^{b}$ to the quadruple $\left(T_{p} N, T_{p} M, h_{p}^{M}, R_{p}^{N}\right)$ according to (10) the curvature equations of Gauß and Ricci can be written in a uniform manner for all $u_{1}, u_{2}, v_{1}, v_{2} \in T_{p} M$ and $z_{1}, z_{2} \in \perp_{p} M$ by

$$
\begin{array}{r}
\left\langle R_{p}^{M}\left(u_{1}, u_{2}\right) v_{1}, v_{2}\right\rangle=\left\langle R^{b}\left(u_{1}, u_{2}\right) v_{1}, v_{2}\right\rangle \text { and } \\
\left\langle R_{p}^{\perp M}\left(u_{1}, u_{2}\right) z_{1}, z_{2}\right\rangle=\left\langle R^{b}\left(u_{1}, u_{2}\right) z_{1}, z_{2}\right\rangle .
\end{array}
$$

If $T_{p} M$ is curvature invariant (i.e. $R_{p}^{N}$-invariant), then we even have

$$
\begin{aligned}
& R_{p}^{M}\left(u_{1}, u_{2}\right)=R^{b}\left(u_{1}, u_{2}\right) \mid T_{p} M \quad \text { and } \\
& R_{p}^{\perp M}\left(u_{1}, u_{2}\right)=R^{b}\left(u_{1}, u_{2}\right) \mid \perp_{p} M
\end{aligned}
$$


thus in this situation $h_{p}^{M}$ is $R_{p}^{N}$-semiparallel if and only if for all $u_{1}, u_{2}, v_{1}, v_{2} \in T_{p} M$ we have

$$
R_{p}^{\perp M}\left(u_{1}, u_{2}\right)\left(h_{p}^{M}\left(v_{1}, v_{2}\right)\right)=h_{p}^{M}\left(R_{p}^{M}\left(u_{1}, u_{2}\right) v_{1}, v_{2}\right)+h_{p}^{M}\left(v_{1}, R_{p}^{M}\left(u_{1}, u_{2}\right) v_{2}\right),
$$

which is the usual characterization in case of curvature constant spaces $N$.

(b) Let $V$ and $W$ be as in Definition 1 and put $b:=\langle\cdot, \cdot\rangle z \in \mathrm{L}^{2}\left(W, W^{\perp}\right)$ and $\omega:=\|z\|$ with some vector $z \in W^{\perp}$. Then for every unit vector $u \in W$ we get $A:=A_{u}^{b}=-u \wedge z: v \mapsto$ $\langle v, u\rangle z-\langle v, z\rangle u$; in particular, $A u=z, A z=-\omega^{2} u$ and $A v=0$ for $v \in \operatorname{span}\{u, z\}^{\perp}$. Thus the orbits of these vectors with respect to the 1-paramater subgroup $t \mapsto \operatorname{Exp}(t A)$ are $\beta_{u}^{A}(t)=\cos (\omega t) u+\omega^{-1} \sin (\omega t) z, \quad \beta_{z}^{A}(t)=\cos (\omega t) z-\omega \sin (\omega t) u$ and $\beta_{v}^{A} \equiv v$. If furthermore $R$ is an algebraic curvature tensor on $V$, then we get $R^{b}\left(u_{1}, u_{2}\right)=$ $R\left(u_{1}, u_{2}\right)+\langle z, z\rangle u_{1} \wedge u_{2}$ for all $u_{1}, u_{2} \in W$; if in addition $W$ is $R$-invariant, $b$ therefore is $R$-semiparallel if and only if $R\left(u_{1}, u_{2}\right) z=0$ for all $u_{1}, u_{2} \in W$.

Lemma 1. Let an element $A \in \operatorname{End}_{-}(V)$ and a unit vector $v \in V$ be given and put $r:=$ $\operatorname{dim} V(A, v)$. Then with the notations of Definition 1(c) the following assertions (a) - (c) are true:

(a) There exists an orthonormal basis $\left(a_{1}, \ldots, a_{r}\right)$ of $V(A, v)$ with $a_{1}=v$ and numbers $\varkappa_{1}, \ldots, \varkappa_{r-1} \in \mathbb{R}_{+}$such that

$$
A a_{i}=-\varkappa_{i-1} a_{i-1}+\varkappa_{i} a_{i+1} \text { for } i=1, \ldots, r
$$

holds with $a_{0}:=a_{r+1}:=0$ and $\varkappa_{0}:=\varkappa_{r}:=0$. The vectors $a_{1}, \ldots, a_{r}$ and numbers $\varkappa_{1}, \ldots, \varkappa_{r-1}$ are uniquely determined by the pair $(A, v)$.

(b) $V(A, v)=\operatorname{span}\left\{A^{k} v \mid k=0, \ldots, r-1\right\}=\operatorname{span}\left(\beta_{v}^{A}(\mathbb{R})\right)$

$$
=\operatorname{Exp}(t A)(V(A, v))
$$

(c) With the notations of (a) the following holds: The functions $y_{i}=\operatorname{Exp}(t A) a_{i}(1 \leq i \leq r)$ solve the initial value problem

$$
y_{i}^{\prime}=-\varkappa_{i-1} y_{i-1}+\varkappa_{i} y_{i+1} \quad \text { and } y_{i}(0)=a_{i} \quad \text { for } \quad i=1, \ldots, r
$$

with $y_{0}:=y_{r+1}:=0$; notice, $\beta_{v}^{A}=y_{1}$.

Proof. For (a). Differential geometers are familiar with the proof from Frenet theory of curves: Put $a_{1}:=v$. Then $A a_{1} \perp a_{1}$ and for $r>1$ one has $\varkappa_{1}:=\left\|A a_{1}\right\|>0$, put $a_{2}:=\varkappa_{1}^{-1} A a_{1}$. Then $A a_{2}+\varkappa_{1} a_{1} \perp a_{1}, a_{2}$ and for $r>2$ one has $\varkappa_{2}:=\left\|A a_{2}+\varkappa_{1} a_{1}\right\|>0$, put $a_{3}:=$ $\varkappa_{2}^{-1}\left(A a_{2}+\varkappa_{1} a_{1}\right)$. Continuing this procedure the orthonormal basis $\left(a_{1}, \ldots, a_{r}\right)$ of $V(A, v)$ and the numbers $\varkappa_{1}, \ldots, \varkappa_{r-1} \in \mathbb{R}_{+}$are constructed. Finally, $A a_{r}+\varkappa_{r-1} a_{r-1} \perp a_{1}, \ldots, a_{r}$; since on the other hand $A a_{r}+\varkappa_{r-1} a_{r-1} \in V(A, v)$, one obtains the last equation $A a_{r}=-\varkappa_{r-1} a_{r-1}$.

For (b). $V(A, v)=\operatorname{span}\left\{A^{k} v \mid k=0, \ldots, r-1\right\}$ is clear from the definition of $V(A, v)$, and $\operatorname{Exp}(t A)(V(A, v))=V(A, v)$ follows from $\operatorname{Exp}(t A)=\sum_{k=0}^{\infty} \frac{1}{k !} \cdot t^{k} A^{k}$; in particular we get $\beta_{v}^{A}(\mathbb{R}) \subset V(A, v)$. Conversely, if $\beta_{v}^{A}(\mathbb{R}) \subset W$ holds for some linear subspace $W \subset V$, then also the derivatives $\left(\beta_{v}^{A}\right)^{(k)}(0)=A^{k} v(k<r)$ are contained in $W$, hence $V(A, v) \subset W$.

For (c). Since $\frac{\mathrm{d}}{\mathrm{d} t} \operatorname{Exp}(t A)=\operatorname{Exp}(t A) A$, the statement follows from (13). 
Lemma 2. Let be given two quadruples $(V, W, b, R)$ and $(\tilde{V}, \tilde{W}, \tilde{b}, \tilde{R})$ as in Definition $1(b)$ and a linear isometry $\varphi: V \rightarrow \tilde{V}$ with $\varphi(W)=\tilde{W}$ and assume that for all $u_{1}, u_{2} \in W$ and $v \in V$ the following holds:

$$
\begin{aligned}
& \tilde{b}\left(\varphi\left(u_{1}\right), \varphi\left(u_{2}\right)\right)=\varphi\left(b\left(u_{1}, u_{2}\right)\right) \quad \text { and } \\
& \tilde{R}\left(\varphi\left(u_{1}\right), \varphi\left(u_{2}\right)\right) \varphi(v)=\varphi\left(R\left(u_{1}, u_{2}\right) v\right) .
\end{aligned}
$$

Then $\tilde{W}$ is $\tilde{R}$-invariant if and only if $W$ is R-invariant; and if the latter is true, then $\tilde{b}$ is $\tilde{R}$-semiparallel if and only if $b$ is R-semiparallel.

Proof. Notice that $\varphi$ is an isomorphism between the two quadruples.

An essential tool in our treatment will be the procedure of envelopping a curve, which is the converse of CARTAN's procedure of developping a curve:

Definition 2 (De- and envelopments). Here we suppose that the manifold $N$ is only equipped with a linear connection $\nabla^{N}$. For every curve $c: J \rightarrow N$ with $0 \in J$ and $q:=c(0)$ we define:

(a) For every vector field $Y: J \rightarrow T N$ along $c$ the function $\hat{Y}: J \rightarrow T_{q} N$ given by

$$
\hat{Y}(t):=\underset{t}{(\|})^{N} Y(t) \quad(\text { see }(1))
$$

is called the backward parallel displacement of $Y$ into $T_{q} N$.

(b) The curve $C: J \rightarrow T_{q} N$ with initial point $C(0)=0$ whose derivative $C^{\prime}$ is the backward parallel displacement of the vector field $\dot{c}$ is called the development of $c$ (see $[\mathrm{KN}]$ vol. 1, p. 130). Conversely, $c$ will be called "the" envelopment of $C$ (see Proposition $2(\mathrm{~b}))$.

Proposition 2 (Envelopments). In the situation of Definition 2 let a point $q \in N$ be given.

(a) If $\hat{Y}$ is the backward parallel displacement of a vector field $Y$ along a curve $c: J \rightarrow N$, then the derivative $\hat{Y}^{\prime}$ is the backward parallel displacement of the covariant derivative $\nabla_{\partial}^{N} Y$. In particular, in a riemannian manifold the Frenet apparatus of a curve "coincides" with the Frenet apparatus of its envelopment (considered as a curve in the euclidean space $\left.T_{q} N\right)$.

(b) For every $\mathrm{C}^{\infty}$-curve $C: J \rightarrow T_{q} N$ with $0 \in J$ and $C(0)=0$ there exists an interval $\tilde{J} \subset J$ with $0 \in \tilde{J}$ and an envelopment $c: \tilde{J} \rightarrow N$ of $C \mid \tilde{J}$, which is maximal and unique in the following sense: If $c^{*}: J^{*} \rightarrow N$ is another envelopment of this kind, then we have $J^{*} \subset \tilde{J}$ and $c^{*}=c \mid J^{*}$.

(c) Let $L$ be a further manifold, $G \subset \mathbb{R} \times L$ an open neighborhood of $\{0\} \times L$ and assume that $J_{\lambda}:=\{t \in \mathbb{R} \mid(t, \lambda) \in G\}$ is an interval for every $\lambda \in L$. Furthermore, let a $\mathrm{C}^{\infty}$-map $F: G \rightarrow T_{q} N$ with $F \mid\left(\{0\}_{\sim} \times L\right) \equiv 0$ be given and denote the maximal envelopment of $C_{\lambda}:=F(\cdot, \lambda)$ by $c_{\lambda}: \tilde{J}_{\lambda} \rightarrow N$ for every $\lambda$. Then $\tilde{G}:=$ $\bigcup_{\lambda \in L}\left(\tilde{J}_{\lambda} \times\{\lambda\}\right)$ is again an open neighborhood of $\{0\} \times L$, and

$$
\tilde{F}: \tilde{G} \rightarrow N,(t, \lambda) \mapsto c_{\lambda}(t) \quad \text { is a } \mathrm{C}^{\infty}-\operatorname{map} .
$$


(d) Suppose $C: J \rightarrow T_{q} N$ is a $\mathrm{C}^{\infty}$-curve with $0 \in J$ and $C(0)=0$, and let $c: \tilde{J} \rightarrow N$ denote its maximal development. Then $c$ is also maximal in the following sense: If $c^{*}: J^{*} \rightarrow N$ is a curve defined on some interval $J^{*}$ with $\tilde{J} \subset J^{*} \subset J$ and satisfying $c^{*} \mid \tilde{J}=c$, then $\tilde{J}=J^{*}$.

(e) If $N$ is a riemannian manifold and $\nabla^{N}$ its Levi Civita connection, one can say even more: If $\delta:=\sup \tilde{J}<\sup J$ holds in the assertion of (b), then for every compact set $K \subset N$ there exists a parameter $s \in[0, \delta[$ such that $K \cap c([s, \delta[)=\varnothing$.

Proof. (a) is deduced easily by use of a frame field of $T N$ along $c$.

Although assertion (b) is well known, we indicate its proof. Let $\pi: \mathrm{L}(N) \rightarrow N$ denote the frame bundle of $N$. As in $[\mathrm{KN}]$,Vol.1,p. 55 the elements of the fibre $\mathrm{L}_{q}(N)$ are considered as isomorphisms $u: \mathbb{R}^{n} \rightarrow T_{q} N$. In order to prove (b) we choose a frame $u_{0} \in \mathrm{L}_{q}(N)$, introduce the vector field $X: J \times \mathrm{L}(N) \rightarrow T N,(t, u) \mapsto u \circ u_{0}^{-1}\left(C^{\prime}(t)\right)$ and its horizontal lift $J \times \mathrm{L}(N) \rightarrow T \mathrm{~L}(N)$ which on $\mathrm{L}(N)$ describes a time depending differential equation. Then a curve $E: J^{*} \rightarrow \mathrm{L}(N)$ with $E(0)=u_{0}$ is a solution of this differential equation if and only if $E$ is horizontal and $\pi \circ E$ is an envelopment of $C$. This implies (b).

For (c) one works with the vector field $X: G \times \mathrm{L}(N) \rightarrow T N,(t, \lambda, u) \mapsto u \circ u_{0}^{-1}\left(F_{*} \frac{\partial}{\partial t}(t, \lambda)\right)$ and the corresponding time and parameter depending differential equation.

For $(\mathrm{d})$, let $\delta:=\sup \tilde{J}$ and assume $\delta<\sup J^{*}$. Let $E$ resp. $E^{*}$ denote the horizontal lift to $L(N)$ of $c$ resp. $c^{*}$ with $E(0)=E^{*}(0)$; thus $\lim _{t \rightarrow \delta} E(t)=E^{*}(\delta)$ exists. Now by the proof of (b), $E$ is the maximal solution of a time depending differential equation described by a vector field $J \times L(N) \rightarrow T L(N)$. Since $\delta<\sup J, \lim _{t \rightarrow \delta} E(t)$ can not exist in $L(N)$ by means of the theory of ordinary differential equations. This is a contradiction to the assumption $\delta<\sup J^{*}$. Thus we see $\tilde{J}=J^{*}$.

For (e) one modifies the proof of (b) by substituting the bundle $\mathrm{L}(N)$ by the orthonormal frame bundle of $N$. Then $\pi$ is a proper map and the assertion follows from the behavior of solutions of differential equations.

Proposition 3 (Total completeness). Every complete riemannian manifold $N$ and every reductive homogeneous space $N$ (with its canonical connection) is totally complete, that means, for every $p \in N$ and every curve $C: J \rightarrow T_{p} N$ with $0 \in J$ and $C(0)=0$ the maximal envelopment of $C$ is defined on the entire interval $J$.

For the case of reductive homogeneous space see also [W]. Notice also that symmetric spaces are reductive homogeneous spaces.

Proof. Let a curve $C: J \rightarrow T_{p} N$ with $0 \in J$ and $C(0)=0$ be given. - In the case of a complete riemannian manifold let denote the maximal envelopment of $C$ by $c: \tilde{J} \rightarrow N$ and suppose $\delta:=\sup \tilde{J}<\sup J$. Because of $\left\|C^{\prime}(t)\right\|=\|\dot{c}(t)\|, \quad c([0, \delta[)$ is contained in the ball $B_{r}(q) \subset N$ with $r:=\operatorname{length}(C \mid[0, \delta])$. Since $N$ is complete, the closed ball $\overline{B_{r}(q)} \subset N$ is compact, and we get a contradiction to Proposition 2(e).

Now let $N$ be a reductive homogeneous space and choose the above point $p \in N$ as reference point. The reductive homogenous structure of $N$ is given by a transitive action $\phi: G \times N \rightarrow N$ 
of some Lie group G such that the Lie algebra $\mathfrak{g}$ of $G$ splits in the direct sum $\mathfrak{k} \oplus \mathfrak{m}$, where $\mathfrak{k}$ is the Lie algebra of the isotropy group of $\phi$ corresponding to $p$ and $\mathfrak{m}$ is some $\operatorname{Ad}(K)$-invariant subspace of $\mathfrak{g}$; here we consider $\mathfrak{g}$ as the Lie algebra of the left invariant vector fields of $G$. Then $\phi^{p}: G \rightarrow M, g \mapsto \phi(g, p)$ is a principal fibre bundle over $N$ with structure group $K$, and

$$
\theta: \mathfrak{m} \rightarrow T_{p} N, X \mapsto \phi_{*}^{p} X_{e}
$$

is an isomorphism. In order to describe the the canonical linear connection ${ }^{2} \nabla^{N}$ of $N$ we equip the principal fibre bundle $\phi^{p}$ with the $G$-invariant connection $\mathcal{H}$ defined by

$$
\mathcal{H}_{g}:=\left\{X_{g} \mid X \in \mathfrak{m}\right\}
$$

Since the tangent bundle $T N$ is associated with $\phi^{p}$ via the map

$$
\rho: \mathrm{G} \times T_{p} N \rightarrow T N,(g, v) \mapsto \phi_{g *} v,
$$

the connection $\mathcal{H}$ induces also a linear connection $\nabla^{N}$ on the tangent bundle, namely the canonical connection; it should be mentioned that it is independent of the choice of the reference point $p$. According to (1) and the construction of $\nabla^{N}$ (see [KN], Vol. 1, p. 87) it has the following characterizing property: For every $\mathcal{H}$-horizontal curve $\gamma: J \rightarrow G$ with $\gamma(0)=e$ the $\nabla^{N}$-parallel displacement in $T N$ along $c:=\phi^{p} \circ \gamma$ is given by

$$
\stackrel{t}{\| c})^{N}=\phi_{\gamma(t) *}: T_{p} N \rightarrow T_{c(t)} N
$$

Now, starting with the given curve $C: J \rightarrow T_{p} N$ we define the function

$$
A:=\theta^{-1} \circ C^{\prime}: J \rightarrow \mathfrak{m} .
$$

It is well known (compare with the analogous situation in [KN], Vol.1, Lemma on p. 69) that there exists a curve $\gamma: J \rightarrow G$ (defined on the entire interval $J$ ) satisfying the differential equation

$$
\dot{\gamma}(t)=A(t)_{\gamma(t)} \text { for all } t \in J \text { with the initial value } \gamma(0)=e .
$$

The proof will be complete when we will have shown that

$$
c:=\phi^{p} \circ \gamma: J \rightarrow N
$$

is the envelopment of $C$. This is done by means of the Equations (16) - (18) and the fact that $\gamma$ is $\mathcal{H}$-horizontal by construction:

$$
\begin{aligned}
\stackrel{t}{(\| c})^{N} C^{\prime}(t) & =\phi_{\gamma(t) *} C^{\prime}(t)=\phi_{\gamma(t) *} \phi_{*}^{p} A(t)_{e}=\left(\phi_{\gamma(t)} \circ \phi^{p}\right)_{*} A(t)_{e} \\
& =\left(\phi^{p} \circ L_{\gamma(t)}\right)_{*} A(t)_{e}=\phi_{*}^{p} L_{\gamma(t) *} A(t)_{e}=\phi_{*}^{p} A_{\gamma(t)}=\phi_{*}^{p} \dot{\gamma}(t)=\dot{c}(t) .
\end{aligned}
$$

Inspired by the treatment $[\mathrm{NY}]$ of circles in arbitrary riemannian manifolds we now will define helical arcs in the following way.

\footnotetext{
${ }^{2}$ I have learned this construction from Prof. H. Reckziegel. For another description see [KN] Vol.2, p. 190.
} 
Definition 3 (Helical arcs). In a riemannian manifold $N$ a curve $c: J \rightarrow N$ with $0 \in J$, $q:=c(0)$ and $u:=\dot{c}(0)$ will be called a helical arc, if there exists some $A \in \operatorname{End}_{-}\left(T_{q} N\right)$ such that $c$ is the envelopment of $C \mid J$ where $C: \mathbb{R} \rightarrow T_{q} N$ is the curve with $C(0)=0$ and $C^{\prime}(t)=\operatorname{Exp}(t A) u$, in other words,

$$
\left.\left.\dot{c}(t)=\underset{0}{\left(\left(\|_{0}\right.\right.}\right)^{N} \circ \operatorname{Exp}(t A)\right) u \text { for all } t \in J .
$$

Note that $\|\dot{c}(t)\| \equiv\|u\|$. The rank of the orbit $\beta_{u}^{A}: t \mapsto \operatorname{Exp}(t A) u$ is also called the rank of the helical arc $c$ (see Definition 1(c)). If $W$ is a proper subspace of $T_{q} N$ containing the vector $u, b \in \mathrm{L}^{2}\left(W, W^{\perp}\right)$ and $A=A_{u}^{b}$ (see Definition $1(\mathrm{a})$ ), we will say that $c$ is generated by the quadruple $(q, W, b, u)$. If $c$ is the maximal envelopment of $C$ in $N$, then $c$ is called the maximal helical arc associated with the pair $(u, A)$.

Remark 1. (a) In a riemannian manifold $N$ a unit speed curve $c: J \rightarrow N$ with $0 \in J$ is a helical arc of rank $r$ if and only if its Frenet equations are

$$
\nabla_{\partial}^{N} E_{i}=-\varkappa_{i-1} E_{i-1}+\varkappa_{i} E_{i+1} \quad \text { for } \quad i=1, \ldots, r
$$

with $E_{1}=\dot{c}$, suitable numbers $\varkappa_{1}, \ldots, \varkappa_{r-1} \in \mathbb{R}_{+}, \varkappa_{0}:=\varkappa_{r}:=0$ and $E_{0}:=E_{r+1}:=0$.

(b) If $N$ is complete, then every maximal helical arc of $N$ is defined on $\mathbb{R}$.

Proof. Since, according to Proposition 2(a), $c$ has the same Frenet apparatus as its development $C$, statement (a) follows from Lemma 1(c). For (b) take notice of Proposition 3(a).

Example 3. The helical arcs of rank 0 are the constant curves. Helical arcs of rank 1 are the geodesics with velocity different from 0 ; and the unit speed helical arcs of rank 2 are the circular arcs (see Example 1(b)), which are no geodesics; this follows from Remark 1(a); see also [NY]. It should also be noticed that if $u$ is a unit vector then in the situation (19) $c$ has rank 2 if and only if $\left(u, \omega^{-1} A u\right)$ is an orthonormal basis of $V(A, u)$ for some $\omega \in \mathbb{R}_{+}$; then we are in the situation of Example 2(b) with $z:=A u$.

Definition 4 ( $M$-split-parallel displacement). Let $M$ be a submanifold of $N, c: J \rightarrow M$ a curve with $0 \in J$ and $p:=c(0)$. Then the $M$-split-parallel displacement $(\varphi(t))_{t \in J}$ of $T_{p} N$ along the curve $c$ is the family of linear isometries $\varphi(t): T_{p} N \rightarrow T_{c(t)} N$ characterized by the following properties:

- $\varphi(t) \mid T_{p} M$ is the parallel displacement $\left.\underset{0}{\stackrel{t}{\|}}\right)^{M}: T_{p} M \rightarrow T_{c(t)} M$ in $M$ along $c$ (in particular $\left.T_{c(t)} M=\varphi(t)\left(T_{p} M\right)\right)$ and

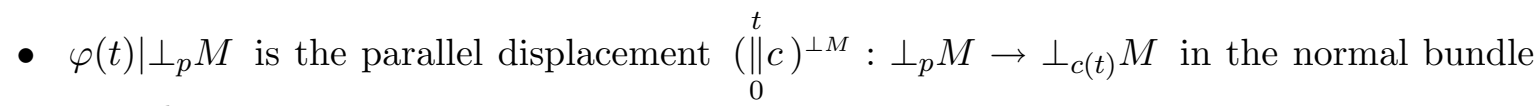
$\perp M$ along $c$.

Example 4. By means of the $M$-split-parallel displacement $(\varphi(t))_{t \in J}$ of $T_{p} N$ along the curve $c$ it is now easy also to describe the parallel displacement of $h_{p}^{M}$ (with respect to $\bar{\nabla}$, see (2) and also (81) and (68)) along $c$ to the point $c(t)$; it is given by

$$
\varphi(t) \circ h_{p}^{M} \circ\left(\varphi(t)^{-1} \times \varphi(t)^{-1}\right) \mid T_{c(t)} M \times T_{c(t)} M .
$$


The next proposition shows how the twisting of the tangent spaces of a submanifold $M \subset N$ along a curve $c: J \rightarrow M$ (described by the isometries $\varphi(t))$ is related to the second fundamental form of $M$.

Proposition 4 (Relation between the $\boldsymbol{M}$-split-parallel displacement and $\boldsymbol{h}^{\boldsymbol{M}}$ ). In the situation of Definition 4 define $W:=T_{p} M$, let $\ell(t) \in \mathrm{L}\left(W, W^{\perp}\right)$ be the canonical backward parallel transport of $h_{c(t)}^{M}(\dot{c}(t), \cdot)$ along $c$ to $p$, i.e.,

$$
\varphi(t) \circ \ell(t) v:=h_{c(t)}^{M}(\dot{c}(t), \varphi(t) v)
$$

and define

$$
\left.J \ni t \mapsto A(t):=A_{\ell(t)} \in \mathbb{M}_{W} \quad \text { (see Definition 1(a) and Formula }(7)\right) .
$$

Then the function

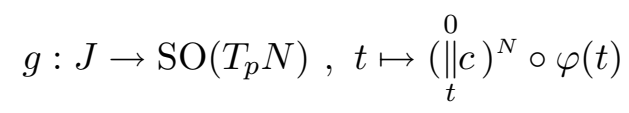

is the solution of the linear differential equation

$$
g^{\prime}(t)=g(t) \circ A(t) \quad \text { with } \quad g(0)=\mathrm{id}_{T_{p} N} .
$$

Proof. If we put $b(t):=h_{c(t)}^{M}$, then we derive easily

$$
\varphi(t) \circ A(t)=A_{\dot{c}(t)}^{b(t)} \circ \varphi(t)
$$

Now we abbreviate $\psi(t):=\underset{0}{\stackrel{t}{\| c}})^{N}$, fix a vector $v \in T_{p} N$ and define the vector field $Y \in \Gamma_{c}(T N)$ by $Y(t):=\varphi(t) v=\psi(t) \circ g(t) v$. Obviously $\hat{Y}: t \mapsto g(t) v \in T_{p} N$ is the backward parallel displacement of $Y$ into $T_{p} N$ (see Definition 2); thus we get from Proposition 2(a)

$$
\nabla_{\partial}^{N} Y(t)=\psi(t) \hat{Y}^{\prime}(t)=\psi(t) \circ g^{\prime}(t) v .
$$

If we write $Y=Y^{\top}+Y^{\perp}$ according to the splitting $T_{c(t)} N=T_{c(t)} M \oplus \perp_{c(t)} M$, we obtain from the definition of the $M$-split-parallel displacement that $Y^{\top}$ is a parallel vector field of $M$ and $Y^{\perp}$ a parallel normal field of $M$. Using the equations of Gauß and Weingarten in combination with Example 2(a) and (23) we get

$$
\nabla_{\partial}^{N} Y(t)=A_{\dot{c}(t)}^{b(t)} Y(t)=\varphi(t) \circ A(t) v=\psi(t) \circ g(t) \circ A(t) v
$$

Thus, by means of (24) we see that (22) is satisfied.

Remark 2 (The twisting of the tangent spaces of a submanifold). In the situation of Proposition 4 the tangent space $T_{c(t)} M$ is obtained from $g(t)(W)$ merely by parallel transport in $N$ along $c$. Thus the function $g$ describes the twisting of the tangent spaces of $M$ along $c$. On the other hand, because of $A(t) \in \mathbb{M}_{W}$ and (22) the curve

$$
J \rightarrow \mathrm{G}_{m}\left(T_{p} N\right), t \mapsto g(t)(W)
$$


is the envelopment of the curve

$$
C: J \rightarrow T_{W} \mathrm{G}_{m}\left(T_{p} N\right), t \mapsto \int_{0}^{t} \Theta_{W}(A(s)) \mathrm{d} s ;
$$

here we consider the Graßmann manifold $\mathrm{G}_{m}\left(T_{p} N\right)$ as symmetric space with its canonical linear connection (see also Proposition 3(b) and its proof). Taking the definition of $A(t)$ in account we recognize how the second fundamental form influences the twisting of the tangent spaces of $M$ along $c$; see also Proposition 1.

Besides, it should be mentioned that

the curve $c$ (in Proposition 4) is a geodesic of $M$ if and only if the development $C$ of $c$ in $T_{p} N$ satisfies $C^{\prime}(t)=g(t) \dot{c}(0)$ for all $t \in J$.

Indeed, both statements are equivalent to $\forall t \in J: \dot{c}(t)=\varphi(t) \dot{c}(0)$.

Proposition 5 (Geometry of II-parallel submanifolds). If $M$ is a II-parallel submanifold of $N$, then the following assertions are true:

(a) Every tangent space $T_{p} M$ is curvature invariant, i.e., it is $R_{p}^{N}$-invariant.

(b) For every $p \in M$ the second fundamental form $h_{p}^{M}$ is $R_{p}^{N}$-semiparallel.

(c) Every geodesic $c: J \rightarrow M$ of $M$ is a helical arc in $N$ and the tangent spaces $T_{c(t)} M$ rotate uniformly; in more details: If we assume $0 \in J$, put $p:=c(0), u:=\dot{c}(0)$ and $b:=h_{p}^{M}$, then the $M$-split-parallel displacement of $T_{p} N$ along $c$ is given by

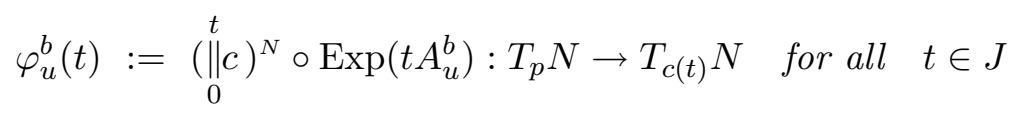

(see Definition 1). In particular, $\dot{c}(t)=\varphi_{u}^{b}(t) u$; hence $c$ is a helical arc generated by the quadruple $\left(p, T_{p} M, h_{p}^{M}, u\right)$. Furthermore, the second fundamental form $h^{M}$ satisfies

$$
h_{c(t)}^{M}\left(\varphi_{u}^{b}(t) v_{1}, \varphi_{u}^{b}(t) v_{2}\right)=\varphi_{u}^{b}(t) b\left(v_{1}, v_{2}\right) \text { for all } v_{1}, v_{2} \in T_{p} M .
$$

Proof. (a) is exactly the Codazzi equation for $M$, and (b) is obtained immediately from $\bar{\nabla} h^{M}=$ 0 by means of (a) and (12). For (c): We use the notations of Proposition 4; in particular, $(\varphi(t))_{t \in J}$ denotes the $M$-split-parallel displacement along $c$. Because $h^{M}$ and $\dot{c}$ are parallel, we find $\ell(t) \equiv \ell(0)=h_{p}^{M}(u, \cdot)=b(u, \cdot)$; consequently we get $A(t) \equiv A_{u}^{b}$. Hence (22) implies $g(t)=\operatorname{Exp}\left(t A_{u}^{b}\right)$ and consequently $\varphi(t)=\varphi_{u}^{b}(t)$. - Assertion (d) follows trivially from (c) and the parallelity of $h^{M}$.

As a corollary of Proposition 5(a) we easily get the following result, which is mentioned (without proof) in [Na1], p. 217:

Corollary 2. If $f: M \rightarrow N$ is a II-parallel immersion into a locally symmetric space $N$, then also $M$ is locally symmetric. 
Proof. Since the local symmetry of a space is a local property, we may suppose that $M$ is a II-parallel submanifold of $N$ and $f$ the inclusion $M \hookrightarrow N$. Let $R^{N, b}$ resp. $R^{M, b}$ denote the (0,4)-tensor on $N$ resp. $M$ associated canonically with the curvature tensor $R^{N}$ resp. $R^{M}$. We must prove $\nabla^{M} R^{M, b}=0$; this means: If $c: J \rightarrow M$ is any curve and $Y_{i} \in \Gamma_{c}(T M)(i=1, \ldots, 4)$ are any parallel vector fields along $c$, we must show $R^{M, b}\left(Y_{1}, Y_{2}, Y_{3}, Y_{4}\right) \equiv$ const. Of course, the proof is based on the Gauß equation:

$$
R^{M, b}\left(Y_{1}, Y_{2}, Y_{3}, Y_{4}\right)=R^{N, b}\left(Y_{1}, Y_{2}, Y_{3}, Y_{4}\right)+F
$$

with the function

$$
F:=\left\langle h^{M}\left(Y_{1}, Y_{4}\right), h^{M}\left(Y_{2}, Y_{3}\right)\right\rangle-\left\langle h^{M}\left(Y_{1}, Y_{3}\right), h^{M}\left(Y_{2}, Y_{4}\right)\right\rangle,
$$

which in fact is constant, as $h^{M}$ is parallel. Thus we get

$$
\begin{aligned}
\left(R^{M, b}\left(Y_{1}, Y_{2}, Y_{3}, Y_{4}\right)\right)^{\prime} & =\left(R^{N, b}\left(Y_{1}, Y_{2}, Y_{3}, Y_{4}\right)\right)^{\prime} \\
& =\left(\nabla_{\dot{c}} R^{N, b}\right)\left(Y_{1}, Y_{2}, Y_{3}, Y_{4}\right)+\sum_{i} R^{N, b}\left(Y_{1}, \ldots, \nabla_{\partial}^{N} Y_{i}, \ldots, Y_{4}\right) .
\end{aligned}
$$

Since the $Y_{i}$ are $\nabla^{M}$-parallel, we have $\nabla_{\partial}^{N} Y_{i}=h^{f}\left(\dot{c}, Y_{i}\right)$. Thus Proposition 5(a) implies for instance:

$$
R^{N, b}\left(Y_{1}, \ldots, Y_{3}, \nabla_{\partial}^{N} Y_{4}\right)=\left\langle R^{N}\left(Y_{1}, Y_{2}\right) Y_{3}, h^{f}\left(\dot{c}, Y_{4}\right)\right\rangle=0 .
$$

Because of the symmetry properties of the curvature tensor we also have

$$
\forall i=1,2,3: \quad R^{N, b}\left(Y_{1}, \ldots, \nabla_{\partial}^{N} Y_{i}, \ldots, Y_{4}\right)=0 .
$$

From (26) we therefore obtain the equation

$$
\left(R^{M, b}\left(Y_{1}, Y_{2}, Y_{3}, Y_{4}\right)\right)^{\prime}=\left(\nabla_{\dot{c}} R^{N, b}\right)\left(Y_{1}, Y_{2}, Y_{3}, Y_{4}\right),
$$

which holds in every riemannian manifold $N$. In the case of a locally symmetric space $N$ the right hand side of the previous equation vanishes, and thus the corollary is proved.

In the following I will weaken the hypothesis of Proposition 5 and will show that nevertheless an essential part of assertion (c) keeps valid.

Proposition 6. For every m-dimensional submanifold $M \subset N$ the following assertions are equivalent:

(a) $\left(\bar{\nabla}_{u} h^{M}\right)(u, v)=0$ holds for all $p \in M$ and $u, v \in T_{p} M$.

(b) For every geodesic $c: J \rightarrow M$ of $M$ with $0 \in J$ and $p:=c(0)$ the $M$-split-parallel displacement $(\varphi(t))_{t \in J}$ of $T_{p} N$ along $c$ is given by

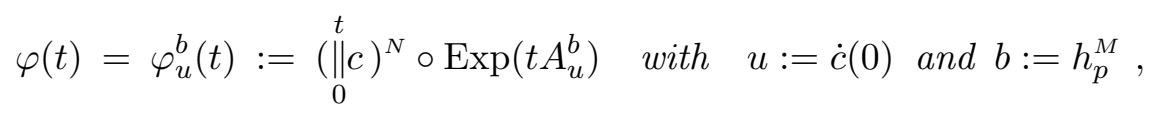

$c$ is the helical arc generated by the quadruple $\left(p, T_{p} M, h_{p}^{M}, u\right)$, and the second fundamental form $h^{M}$ satisfies

$$
h_{c(t)}^{M}\left(\dot{c}(t), \varphi_{u}^{b}(t) v\right)=\varphi_{u}^{b}(t) b(u, v) \text { for all } v \in T_{p} M .
$$


(c) The Gauß map $\xi^{M}: M \rightarrow \hat{N}:=\mathrm{G}_{m}(T N), p \mapsto T_{p} M$ is totally geodesic ${ }^{3}$ with respect to the covariant derivative $\hat{\nabla}$ introduced in Section 6.

Proof. Let be given a point $p \in M$ and a vector $u \in T_{p} M$ and let $c: J \rightarrow M$ be a geodesic of $M$ with $0 \in J$ and $\dot{c}(0)=u$. We use the notations of Proposition 4 with respect to $c$. - As $\dot{c}$ and $t \mapsto \varphi(t) v$ are parallel vector fields along $c$ in $M$ for all $v \in T_{p} M$, we get

$$
\nabla_{\partial}^{\perp{ }_{\partial}}\left(t \mapsto h_{c(t)}^{M}(\dot{c}(t), \varphi(t) v)\right)=\left(t \mapsto\left(\bar{\nabla}_{\dot{c}(t)} h^{M}\right)(\dot{c}(t), \varphi(t) v)\right)
$$

Therefore, the following sequence of equivalences is true:

$$
\begin{aligned}
& \left(\bar{\nabla}_{\dot{c}(t)} h^{M}\right)(\dot{c}(t), v)=0 \quad \text { for all } t \in J \text { and } v \in T_{c(t)} M \\
& \Longleftrightarrow t \mapsto h_{c(t)}^{M}(\dot{c}(t), \varphi(t) v) \text { is a parallel normal field for every } v \in T_{p} M \\
& \Longleftrightarrow \text { the function } \ell \text { defined in }(20) \text { is constant } \equiv h_{p}^{M}(u, \cdot) \\
& \Longleftrightarrow \text { the function } A \text { defined in }(21) \text { is constant } \equiv A_{u}^{b} \text { with } b:=h_{p}^{M} \\
& \Longleftrightarrow \text { the solution } g \text { of the differential Equation }(22) \text { is given by } g(t)=\operatorname{Exp}\left(t A_{u}^{b}\right) \\
& \Longleftrightarrow \text { the } M \text {-split-parallel displacement of } T_{p} N \text { along } c \text { is }\left(\varphi_{u}^{b}(t)\right)_{t \in J} .
\end{aligned}
$$

Therefrom the equivalence "(a) $\Leftrightarrow(\mathrm{b})$ " can be read off. Furthermore, in Theorem 6 we will learn that the second fundamental form $h^{\xi}$ of the Gauß map $\xi=\xi^{M}$ is related to $\bar{\nabla} h^{M}$ by

$$
h_{p}^{\xi}(u, v)=\Theta_{\xi(p)}\left(A_{v}^{b}\right) \quad \text { with } \quad b:=\bar{\nabla}_{u} h^{M} \quad \text { for all } \quad u, v \in T_{p} M(p \in M) .
$$

Therefore assertion (a) is equivalent to $h_{p}^{\xi}(u, u)=0$ for all $u \in T_{p} M(p \in M)$, which implies the total geodesy of $\xi$.

Remark 3. The equivalence "(a) $\Leftrightarrow$ (c)" of Proposition 6 is some analogue of Corollary 10 .

Corollary 3. A submanifold is II-parallel if and only if all its tangent spaces are curvature invariant and one of the three equivalent conditions (a) - (c) in Proposition 6 holds.

Proof. Because of Proposition 5(a) we can start with a submanifold $M$ whose tangent spaces are curvature invariant. Then according to the equation of Codazzi we have

$$
\left(\bar{\nabla}_{u} h^{M}\right)(v, w)=\left(\bar{\nabla}_{v} h^{M}\right)(u, w) \quad \text { for all } \quad u, v, w \in T_{p} M(p \in M) .
$$

In this situation the II-parallelity of $M$ is equivalent to condition (a) of Proposition 6.

Remark 4. In spaces of constant curvature all submanifolds are curvature invariant; thus here each of the conditions (a) - (c) of Proposition 6 is equivalent to the II-parallelity of $M$. In complex space forms of non-vanishing curvature, the curvature invariant submanifolds are exactly the complex and the totally real ones; see $[\mathrm{CO}]$ p.260.

\footnotetext{
${ }^{3}$ Notice, every affine immersion $\xi: M \rightarrow \hat{N}$ is also totally geodesic, that means, the image $\xi \circ c$ of any geodesic $c$ of $M$ is a geodesic of $\hat{N}$. But since $\hat{\nabla}$ has torsion, a totally geodesic immersion $\xi: M \rightarrow \hat{N}$ may fail to be affine, that means: there may exist a parallel vector field $Y$ in $M$ along some curve $c$, whose image $\xi_{*} Y$ is not parallel in $\hat{N}$.
} 
There is a generalization of the argument " $\bar{\nabla}_{\dot{c}} h^{M}(\dot{c}, \cdot)=0 \Rightarrow A(t) \equiv A_{u}^{b}$ " of the proof of the implication "(a) $\Rightarrow(\mathrm{b})$ " of Proposition 6:

Proposition 7. Let $M \subset N$ be a submanifold with the following property: There exists some $k \in\{0,1,2, \ldots\}$ such that the $k$-th covariant derivative $\bar{\nabla}^{[k]} h^{M}$ of the second fundamental form $h^{M}$ (see the proof for more details) satisfies ${ }^{4}$

$$
\forall u \in T M: \quad\left(\bar{\nabla}^{[k]} h^{M}\right)(\underbrace{u, \ldots, u}_{k+1 \text { times }}, \cdot)=0 .
$$

If in the situation of Proposition $4 c$ is a geodesic with initial velocity $u:=\dot{c}(0)$, then the function $J \ni t \mapsto A(t)$ is the restriction of the polynomial $\sum_{j=0}^{k-1} t^{j} \cdot A_{j}: \mathbb{R} \rightarrow \mathbb{M}_{W}$, whose coefficients $A_{j} \in \mathbb{M}_{W}$ are given by $A_{j}:=A_{\ell_{j}}$ with $\ell_{j}:=\frac{1}{j !} \cdot \bar{\nabla}^{[j]} h^{M}(u, \ldots, u, \cdot) \in \mathrm{L}\left(T_{p} M, \perp_{p} M\right)$ (according to Definition 1(a)).

Proof. In order to explain first the situation in more detail, let us define for arbitrary $j$ the covariant derivative $\bar{\nabla} S \in \Gamma\left(\mathrm{L}^{j+1}(T M, \perp M)\right)$ for sections $S$ of the bundle $\mathrm{L}^{j}(T M, \perp M)$ by the same recipe as for the second fundamental form of $M$, that means

$$
(\bar{\nabla} S)\left(X_{0}, \ldots, X_{j}\right):=\nabla^{\perp M} X_{0}\left(S\left(X_{1}, \ldots, X_{j}\right)\right)-\sum_{i=1}^{j} S\left(X_{1}, \ldots, \nabla^{M} X_{0} X_{i}, \ldots, X_{j}\right) .
$$

For vector fields $Y_{1}, \ldots, Y_{k} \in \Gamma_{c}(T M)$ one calculates

$$
(\bar{\nabla} S)\left(\dot{c}, Y_{1}, \ldots, Y_{j}\right)=\nabla_{\partial}^{\perp M_{\partial}}\left(S\left(Y_{1}, \ldots, Y_{j}\right)\right)-\sum_{i=1}^{j} S\left(Y_{1}, \ldots, \nabla_{\partial}^{M_{\partial}} Y_{i}, \ldots, Y_{j}\right) .
$$

If in particular $c$ is a geodesic of $M$ and $Y \in \Gamma_{c}(T M)$ is $\nabla^{M}$-parallel, we get

$$
\nabla^{\perp M_{\partial}}(S(\dot{c}, \ldots, \dot{c}, Y))=(\bar{\nabla} S)(\dot{c}, \ldots, \dot{c}, Y) .
$$

Now, according to definition (28) we can define the $j$-th derivative $\bar{\nabla}^{[j]} h^{M}$ of the second fundamental form $h^{M} \in \Gamma\left(\mathrm{L}^{2}(T M, \perp M)\right.$ by

$$
\bar{\nabla}^{[j]} h^{M}:=\underbrace{\bar{\nabla} \cdots \bar{\nabla}}_{j \text { times }} h^{M} .
$$

By means of Formula (29) we derive by induction

$$
\left(\bar{\nabla}^{[j]} h^{M}\right)(\underbrace{\dot{c}, \ldots, \dot{c}}_{j+1 \text { times }}, Y)=\underbrace{\nabla_{\partial M}^{\perp M} \cdots \nabla_{\partial}^{\perp M}}_{j \text { times }}\left(h^{M}(\dot{c}, Y)\right)
$$

indeed:

$$
\left(\bar{\nabla}^{[j+1]} h^{M}\right)(\underbrace{\dot{c}, \ldots, \dot{c}}_{j+2 \text { times }}, Y) \underset{(29)}{=} \nabla^{\perp M}{ }_{\partial}\left(\left(\bar{\nabla}^{[j]} h^{M}\right)(\underbrace{\dot{c}, \ldots, \dot{c}}_{j+1 \text { times }}, Y)\right)=\ldots
$$

Now we go into Proposition 4 , but with a geodesic $c$, and abbreviate $W:=T_{p} M$. If then $\ell: J \rightarrow \mathrm{L}\left(W, W^{\perp}\right)$ is defined according to Formula (20), then for every $v \in W$

\footnotetext{
${ }^{4}$ For $k=0$ condition $(27)$ means $h^{M} \equiv 0$, i.e., that $M$ is totally geodesic; in this case we get $A(t) \equiv 0$.
} 
the function $t \mapsto \ell(t) v$ is the backward parallel transport of $h_{c(t)}^{M}(\dot{c}(t), \varphi(t) v)$ in the normal bundle $\perp M$ along $c$ to $p$. Therefore, because of (30) (applied to the parallel field $Y: t \mapsto \varphi(t) v)$ ) we see that the ordinary $j$-th derivative $t \mapsto \ell^{(j)}(t) v$ of the function $t \mapsto \ell(t) v$ is the backward parallel transport of $\left(\bar{\nabla}^{[j]} h^{M}\right)(\dot{c}, \ldots, \dot{c}, Y)$ in the normal bundle $\perp M$ along $c$. Hence, hypothesis (27) implies $\ell^{(k)} \equiv 0$. Consequently, $\ell$ is a restriction of the polynomial $\sum_{j=0}^{k-1} t^{j} \cdot \ell_{j}$ with $\ell_{j}:=\frac{1}{j !} \cdot \ell^{(j)}(0)=\frac{1}{j !} \cdot \bar{\nabla}^{[j]} h^{M}(u, \ldots, u, \cdot)$ $\in \mathrm{L}\left(W, W^{\perp}\right)$. Because of the linearity of the funcion $\mathrm{L}\left(W, W^{\perp}\right) \rightarrow \mathbb{M}_{W}, \lambda \mapsto A_{\lambda}$ the proposition is proved.

Corollary 4 (Rigidity of submanifolds). Let $M$ and $\tilde{M}$ be submanifolds of $N$ both satisfying condition (27) of Proposition 7 for some $k \in\{0,1,2, \ldots\}$, and suppose that there exists a point $p \in M \cap \tilde{M}$ such that $W:=T_{p} M=T_{p} \tilde{M}$ and

$$
\forall u, v \in W: \quad\left(\bar{\nabla}^{[j]} h^{M}\right)(u, \ldots, u, v)=\left(\bar{\nabla}^{[j]} h^{\tilde{M}}\right)(u, \ldots, u, v)
$$

holds for $j=0, \ldots, k-1$, and choose some star shaped neighborhood $U$ of 0 in $W$ such that the exponential maps $\exp _{p}^{M}$ and $\exp _{p}^{\tilde{M}}$ are defined on $U$. Then the neighborhoods $\exp _{p}^{M}(U)$ and $\exp _{p}^{\tilde{M}}(U)$ of $p$ in $M$ resp. $\tilde{M}$ coincide.

Notice that condition (27) is fulfilled with $k=1$ if $M$ and $\tilde{M}$ are II-parallel submanifolds; condition (31) then reduces to $h_{p}^{M}=h_{p}^{\tilde{M}}{ }^{5}$

Proof. Let $u \in W$ be given, put $J:=\{t \in \mathbb{R} \mid t u \in U\}$, consider the geodesics $c: J \rightarrow M$ and $\tilde{c}: J \rightarrow \tilde{M}$ with $\dot{c}(0)=u=\dot{\tilde{c}}(0)$ and construct the functions $A, \tilde{A}: J \rightarrow \mathbb{M}_{W}$ and $g, \tilde{g}: J \rightarrow \mathrm{SO}\left(T_{p} N\right)$ corresponding to this geodesics according to Proposition 4. Because of Proposition 7 and condition (31) we have $A \equiv \tilde{A}$ and therefore also $g \equiv \tilde{g}$ (because of (22)). Assertion (25) then shows that the geodesics $c$ and $\tilde{c}$ have the same development $C$ in $T_{p} N$, namely $C(t)=g(t) u$. This proves $c \equiv \tilde{c}$.

Example 5. As is well known the cylinder $M:=\mathrm{S}^{1} \times \mathbb{R}$ is a II-parallel submanifold of $\mathbb{R}^{3}$. Generically the unit speed geodesics of $M$ are helical arcs in $\mathbb{R}^{3}$ of rank 3. But there are exceptional directions at every point. The unit speed geodesics in direction of the ruling of $M$ are also geodesics in $\mathbb{R}^{3}$ and have rank 1 , therefore; and the unit speed geodesics in perpendicular direction are circles in $\mathbb{R}^{3}$ and have rank 2, therefore. - Since in general the unit speed geodesics of a II-parallel submanifold will be helical arcs in the ambient space of different ranks, the usual Frenet theoretical description of helical arcs (as in Remark 1) is not practical for our work.

Proposition 8 (Helical umbrellas). Let be given a point $p \in N$, a proper linear subspace $W \neq\{0\}$ of $T_{p} N$ and a symmetric bilinear map $b \in \mathrm{L}^{2}\left(W, W^{\perp}\right)$. Then there exists a maximal star shaped neighborhood $D$ of 0 in $W$ such that for each vector $u \in D$ the maximal helical arc $c_{u}$ generated by the quadruples $(p, W, b, u)$ is defined at least on the interval $[0,1]$ and the map

$$
\exp ^{b}: D \rightarrow N, u \mapsto c_{u}(1)
$$

is differentiable. Moreover, there exists a star shaped neighbourhood $U$ of 0 in $D$ such that $\exp ^{b} \mid U$ is an injective immersion, and the "helical umbrella"

$$
M=M_{U}(p, W, b):=\exp ^{b}(U)
$$

\footnotetext{
${ }^{5}$ This special case of Proposition 4 was already proved in Theorem 2 of the article [R].
} 
is a submanifold of $N$. We have $p \in M$, its tangent space resp. second fundamental form at $p$ is given by $T_{p} M=W$ resp. $h_{p}^{M}=b$, and $\left(\bar{\nabla} h^{M}\right)_{p}=0$ holds if and only if $W$ is curvature invariant.

Remark 5. In order to motivate the notation "helical umbrella" for the submanifold $M_{U}(p, W, b)$ we introduce the intervals $J_{u}:=\{t \in \mathbb{R} \mid t u \in U\}$ for all vectors $u \in W$. Then we get

$$
M_{U}(p, W, b)=\bigcup_{u \in W} c_{u}\left(J_{u} \cap[0, \infty[) ;\right.
$$

that means $M_{U}(p, W, b)$ is the union of all "helical rays" $c_{u}\left(J_{u} \cap[0, \infty[)\right.$ emanating from $p$. Of course, for every $s \in \mathbb{R}_{+}$the rays $c_{u}\left(J_{u} \cap\left[0, \infty[)\right.\right.$ and $c_{s u}\left(J_{s u} \cap[0, \infty[)\right.$ coincide. Therefore, it would be possible to restrict the consideration to those rays constructed with a unit vector $u$. But then the generalization to the pseudoriemannian case (described in Section 11) would not be so obvious.

It should also be mentioned that $\left(\exp ^{b} \mid U\right)^{-1}: M_{U}(p, W, b) \rightarrow W$ may be considered as a chart of the helical umbrella and that the Christoffel symbol of this chart vanishes at the center $p$ because of (38).

Proof. We put $V:=T_{p} N$ and define the differentiable map

$$
F: \mathbb{R} \times W \rightarrow V,(t, u) \mapsto C_{u}(t):=\sum_{k=1}^{\infty} \frac{1}{k !} \cdot t^{k}\left(A_{u}^{b}\right)^{k-1} u
$$

Since $A_{s u}^{b}=s A_{u}^{b}, F$ has the following properties:

$$
F(0, u)=C_{u}(0)=0, C_{s u}(t)=C_{u}(s t), C_{u}^{\prime}(t)=\operatorname{Exp}\left(t A_{u}^{b}\right) u, \text { hence } C_{u}^{\prime}(0)=u .
$$

In particular, for every $u \in W$ the maximal envelopment $c_{u}: \tilde{J}_{u} \rightarrow N$ of $C_{u}$ is the maximal helical arc generated by the quadruple $(p, W, b, u)$. According to Proposition $2(\mathrm{c})$ the set $\tilde{G}:=$ $\bigcup_{u \in W}\left(\tilde{J}_{u} \times\{u\}\right)$ is an open neighborhood of $\{0\} \times W$ in $\mathbb{R} \times W$ and $\tilde{F}: \tilde{G} \rightarrow N,(t, u) \mapsto c_{u}(t)$ is a $\mathrm{C}^{\infty}$-map, which because of (32) satisfies:

$$
\tilde{F}(0, u)=p, c_{s u}(t)=c_{u}(s t) \quad \text { and } \quad \dot{c}_{u}(0)=u ;
$$

the second equation is valid for $t \in \tilde{J}_{s u}=s^{-1} \tilde{J}_{u}$ (if $s \neq 0$ ), because the two curves $c_{s u}$ and $t \mapsto c_{u}(s t)$ have the same development. Since $\tilde{J}_{0}=\mathbb{R}, D:=\{u \in W \mid(1, u) \in \tilde{G}\}$ is a star shaped open neighborhood of 0 in $W$ and $\exp ^{b}: D \rightarrow N, u \mapsto \tilde{F}(1, u)=c_{u}(1)$ a $C^{\infty}$-map. Furthermore, because of (33) we obtain

$$
\left(t \in \tilde{J}_{u} \Longleftrightarrow t u \in D\right) \text { and }\left(t \in \tilde{J}_{u} \Longrightarrow c_{u}(t)=\exp ^{b}(t u)\right) .
$$

Now, we get from (33) and (34) $\exp _{*}^{b} u=\dot{c}_{u}(0)=u$ for every $u \in T_{0} W \cong W$; consequently $\exp _{*}^{b} \mid T_{0} W: T_{0} W \rightarrow T_{p} N$ is injective. Therefore, there exists a star shaped neighborhood $U$ of 0 in $D$ such that $\exp ^{b} \mid U$ is an injective immersion and hence $\exp ^{b}(U)$ is a submanifold of $N$.

Obviously we have $p \in M$ and $T_{p} M=W$. Now we calculate the second fundamental form $h_{p}^{M}$ : From (32) we derive $C_{u}^{\prime \prime}(t)=\operatorname{Exp}\left(t A_{u}^{b}\right) A_{u}^{b} u=\operatorname{Exp}\left(t A_{u}^{b}\right) b(u, u)$, hence $C_{u}^{\prime \prime}(0)=b(u, u)$. By means of Proposition 2(a) we therefore obtain

$$
\left(\nabla_{\partial}^{N} \dot{c}_{u}\right)(0)=b(u, u) \in W^{\perp} ;
$$


thus the Gauß equation implies $h_{p}^{M}(u, u)=\left(\left(\nabla_{\partial}^{N} \dot{c}_{u}\right)(0)\right)^{\perp}=b(u, u)^{\perp}=b(u, u)$.

It remains to discuss the assertion on $\bar{\nabla} h^{M}$. If $\left(\bar{\nabla} h^{M}\right)_{p}=0$ holds, then because of the Codazzi equation $W=T_{p} M$ is curvature invariant. - Conversely, let us suppose the curvature invariance of $W$. Then by means of the Codazzi equation $\left(\bar{\nabla} h^{M}\right)_{p}$ is a symmetric 3 -tensor and therefore it is sufficient to prove:

$$
\forall u \in W:\left(\bar{\nabla} h^{M}\right)(u, u, u)=0 .
$$

For that let $u \in W$ be given. Repeating the preceding argumentation we obtain $C_{u}^{\prime \prime \prime}(0)=$ $\left(A_{u}^{b}\right)^{2} u$ and therefore

$$
\left(\nabla_{\partial}^{N} \nabla_{\partial}^{N} \dot{c}_{u}\right)(0)=A_{u}^{b}(b(u, u)) \in W .
$$

Notice that $c_{u}$ is a curve in $M$, but not necessarily a geodesic of $M$. However Equation (35) implies

$$
\left(\nabla_{\partial}^{M} \dot{c}_{u}\right)(0)=0
$$

Using $\dot{c}_{u}(0)=u$ and $(38)$ we get

$$
\bar{\nabla} h^{M}(u, u, u)=\left.\bar{\nabla} h^{M}\left(\dot{c}_{u}, \dot{c}_{u}, \dot{c}_{u}\right)\right|_{0}=\left.\nabla_{\partial}^{\perp M}\left(h^{M}\left(\dot{c}_{u}, \dot{c}_{u}\right)\right)\right|_{0}=W^{\perp} \text {-part of }\left.\nabla_{\partial}^{N}\left(h^{M}\left(\dot{c}_{u}, \dot{c}_{u}\right)\right)\right|_{0} .
$$

Substituting $h^{M}\left(\dot{c}_{u}, \dot{c}_{u}\right)=\nabla_{\partial}^{N} \dot{c}_{u}-\nabla_{\partial}^{M} \dot{c}_{u}$ we get

$$
\bar{\nabla} h^{M}(u, u, u)=W^{\perp} \text {-part of }\left(\nabla_{\partial}^{N} \nabla_{\partial}^{N} \dot{c}_{u}\right)(0)-W^{\perp} \text {-part of }\left(\nabla_{\partial}^{N} \nabla_{\partial}^{M} \dot{c}_{u}\right)(0) .
$$

Now the first term in the sum vanishes by means (37) and for the second we calculate using Equation (38)

$$
W^{\perp} \text {-part of }\left(\nabla_{\partial}^{N} \nabla_{\partial}^{M} \dot{c}_{u}\right)(0)=h^{M}\left(\dot{c}_{u}(0),\left(\nabla_{\partial}^{M} \dot{c}_{u}\right)(0)\right)=0 \text {. }
$$

thereby (36) is verified.

Example 6. If in Proposition 8 we choose $b=0$, then the helical $\operatorname{arcs} c_{u}$ are geodesics of $N$, because then $\left.\dot{c}_{u}(t)=\underset{0}{(\| t} c_{u}\right)^{N} u$; thus we end up with the geodesic umbrella of CARTAN's Theorem; the function $\exp ^{b}$ of the previous proof is the restriction $\exp _{p}^{N} \mid U$ of the exponential map of $N$. If on the other hand in Proposition 8 we choose $b=\langle\cdot, \cdot\rangle z$ with some vector $z \in W^{\perp} \backslash\{0\}$, then the helical arcs $c_{u}$ with $\|u\|=1$ are circular arcs of $N$ with the initial "acceleration" $\left(\nabla_{\partial}^{N} \dot{c}_{u}\right)(0)=z$ (see the Examples $2(\mathrm{~b})$ and 3 ); in this case we end up with the circular umbrella, which was the main tool in the treatment [PR2] on "spherical submanifolds". Finally, if $W$ is the tangent space $T_{p} M$ of a II-parallel submanifold $M$ and $b=h_{p}^{M}$, then for sufficiently small $U$ the helical umbrella of Proposition 8 is an open part of $M$ according to Proposition 5(c).

\section{The main result}

Let be given a riemannian manifold $N$, a point $p \in N$, a proper linear subspace $W \neq\{0\}$ of $T_{p} N$ and a symmetric bilinear map $b \in \mathrm{L}^{2}\left(W, W^{\perp}\right)$. Motivated by Proposition 5 we define in the situation of Proposition 8 for every $u \in D$ 


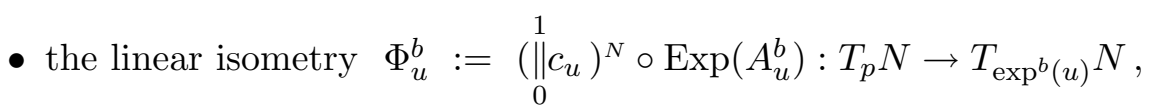

- the linear subspace $W_{u}:=\Phi_{u}^{b}(W) \subset T_{\exp ^{b}(u)} N$ and

- the symmetric bilinear form $b_{u} \in \mathrm{L}^{2}\left(W_{u}, W_{u}^{\perp}\right)$ characterized by

$$
b_{u} \circ\left(\Phi_{u}^{b} \times \Phi_{u}^{b}\right) \mid(W \times W)=\Phi_{u}^{b} \circ b .
$$

Theorem 1 (Main result). The helical umbrella $M_{U}(p, W, b)$ from Proposition 8 has parallel second fundamental form if and only if for every $u \in U$ the following conditions are satisfied:

(CI) $\quad W_{u}$ is curvature invariant and

(SP) $\quad b_{u}$ is $R_{\exp ^{b}(u)}^{N}$-semiparallel.

From Proposition 5 we see that (CI) and (SP) are necessary conditions for the parallelity of the second fundamental form of $M_{U}(p, W, b)$; that these conditions are also sufficient will be proved in Section 8.

Remark 6. For every vector $u \in W$ and every $t \in \mathbb{R}$ with $t u \in D$ let $R_{u}(t)$ be the algebraic curvature tensor on $T_{p} N$ characterized by

$$
\Phi_{t u}^{b}\left(R_{u}(t)\left(v_{1}, v_{2}\right) v_{3}\right)=R_{\exp ^{b}(t u)}^{N}\left(\Phi_{t u}^{b} v_{1}, \Phi_{t u}^{b} v_{2}\right)\left(\Phi_{t u}^{b} v_{3}\right)
$$

According to Lemma 2 the conditions (CI) and (SP) for arbitrary $u \in U$ are equivalent to

(CI') $\quad W$ is $R_{u}(t)$-invariant and

(SP') $\quad b$ is $R_{u}(t)$-semiparallel

for all vectors $u \in W$ and all $t \in J_{u}:=\{t \in \mathbb{R} \mid t u \in U\}$.

Example 7. (a) If $b=0$, then according to Example $6 M_{U}(p, W, b)$ is a geodesic umbrella. Since then $\left.\Phi_{u}^{b}=\underset{0}{\|} \underset{0}{\|} c_{u}\right)^{N}$ and $h_{u}=0$ for every $u \in U$, condition (SP) is satisfied trivially and Theorem 1 is exactly CARTAN's theorem.

(b) If $b=\langle\cdot, \cdot\rangle z$, then according to Example $6 M_{U}(p, W, b)$ is a circular umbrella. If we put $z_{u}:=\Phi_{u}^{b} z=\left(\nabla_{\partial}^{N} \dot{c}_{u}\right)(1)$, then we get $h_{u}=\langle\cdot, \cdot\rangle_{\exp ^{b}(u)} z_{u}$. Therefore, according to Example 2(b) condition (SP) is equivalent to $R^{N}\left(u_{1}, u_{2}\right) z_{u}=0$ for all $u_{1}, u_{2} \in W_{u}$. Thus we have recovered the main result from [PR2] (see p. 200) on the existence of spherical submanifolds. For a comparison of the results it should be mentioned that because of Example 2(b) the space $\left.V_{u}:=\underset{0}{\left(\| c_{u}\right.}\right)^{N}(W \oplus \mathbb{R} z)$ coincides with $W_{u} \oplus \mathbb{R} z_{u}$.

Corollary 5 (II-parallel submanifolds in spaces of constant curvature). If $N$ is a riemannian manifold of constant curvature, then the helical umbrella $M_{U}(p, W, b)$ from Proposition 8 has parallel second fundamental form if and only if $b$ is $R_{p}^{N}$-semiparallel. 
Proof. The assertion follows immediately from Remark 6 , because for all choices of $u$ and $t$ we have $R_{u}(t)=R_{p}^{N}$; and every subspace of $T_{p} N$ is curvature invariant.

I will shed some further light on this situation in Section 4.

At the end of this section we will derive a characterization of those initial data $(p, W, b)$ in a symmetric space $N$, to which there exists a II-parallel submanifold. For that we introduce the vector space $\mathfrak{C}(V)$ of algebraic curvature tensors for an arbitrary euclidean vector space $V$ of dimension $n \geq 2$ and for every subspace $W \subset V$ of dimension $m \in\{1, \ldots, n-1\}$ and every symmetric bilinear map $b \in \mathrm{L}^{2}\left(W, W^{\perp}\right)$ the affine subspace

$\mathfrak{C}(V, W, b):=\{R \in \mathfrak{C}(V) \mid W$ is $R$-invariant and $b$ is $R$-semiparallel $\} \quad$ (see Definition $1(\mathrm{~b}))$;

its associated linear space $\mathfrak{C}(V, W, b)_{L}$ is the space of those algebraic curvature tensors $R$ for which $W$ is $R$-invariant and for all $u_{1}, u_{2}, v_{1}, v_{2} \in W$ the equations

$$
R\left(u_{1}, u_{2}\right)\left(b\left(v_{1}, v_{2}\right)\right)=b\left(R\left(u_{1}, u_{2}\right) v_{1}, v_{2}\right)+b\left(v_{1}, R\left(u_{1}, u_{2}\right) v_{2}\right)
$$

hold. One calculates

$$
d:=\operatorname{dim} \mathfrak{C}(V) \leq \frac{1}{8} n(n-1)\left(n^{2}-n+2\right) .
$$

The space $\mathfrak{C}(V)$ inherits an inner product from $V$ in the usual way; moreover, for every $g \in \mathrm{O}(V)$ and $R \in \mathfrak{C}(V)$ the map $g_{\mathfrak{C}} R:(u, v, w) \mapsto g\left(R\left(g^{-1} u, g^{-1} v\right) g^{-1} w\right)$ is an algebraic curvature tensor again, the map $g_{\mathfrak{C}}: R \mapsto g_{\mathfrak{C}} R$ is an orthogonal transformation of $\mathfrak{C}(V)$ and $\Psi: \mathrm{O}(V) \rightarrow \mathrm{O}(\mathfrak{C}(V)), g \mapsto g_{\mathfrak{C}}$ is a homomorphism of Lie groups. For every $A \in$ End $_{-}(V)$ the image $\Psi \circ \gamma_{A}$ of the 1-parameter subgroup $\gamma_{A}: t \mapsto \operatorname{Exp}(t A) \in \operatorname{SO}(V)$ is given by

$$
\Psi \circ \gamma_{A}(t)=\operatorname{Exp}\left(t A_{\mathfrak{C}}\right)=\sum_{k=0}^{\infty} \frac{1}{k !} t^{k}\left(A_{\mathfrak{C}}\right)^{k},
$$

where $A_{\mathfrak{C}} \in$ End $_{-}(\mathfrak{C}(V))$ is the endomorphism characterized by

$$
A_{\mathfrak{C}} R:(u, v, w) \mapsto A(R(u, v) w)-R(A u, v) w-R(u, A v) w-R(u, v) A w .
$$

Notice that $\Psi_{L}:$ End $_{-}(V) \rightarrow \operatorname{End}_{-}(\mathfrak{C}(V)), A \mapsto A_{\mathfrak{C}}$ is the Lie algebra homomorphism induced by $\Psi$ and End $(V) \times \mathfrak{C}(V) \rightarrow \mathfrak{C}(V),(A, R) \mapsto A_{\mathfrak{C}} R$ the "infinitesimal version" of the action $\Psi$, see $[\mathrm{BD}]$ p. 111.

Now, let $N$ be a symmetric space, $(p, W, b)$ as in Theorem 1 and apply the above consideration to $V:=T_{p} N$. For every $u \in W$ the maximal helical arc $c_{u}$ generated by the quadruple $(p, W, b, u)$ is defined over the whole real line $\mathbb{R}$ (see Proposition 3 ); consequently, the map $\exp ^{b}$ of Proposition 8 is defined on the whole space $W$. Therefore, also the algebraic curvature tensors $R_{u}(t)$ defined in Remark 6 can also be considered for all $(u, t) \in W \times \mathbb{R}$.

As the curvature tensor $R^{N}$ is parallel, for every $u \in W$ and parameter $t \in \mathbb{R}$ we get

$$
R_{u}(t)=\left(\operatorname{Exp}\left(-A_{t u}^{b}\right)\right)_{\mathfrak{C}} R_{p}^{N}=\operatorname{Exp}(t \hat{A}(u)) R_{p}^{N} \quad \text { with } \quad \hat{A}(u)=-\left(A_{u}^{b}\right)_{\mathfrak{C}} .
$$


According to Remark 6 the conditions (CI) and (SP) of Theorem 1 are fulfilled if and only if

$$
R_{u}\left(J_{u}\right) \subset \mathfrak{C}\left(T_{p} N, W, b\right) \text { for every vector } u \in W .
$$

For each $u$ this condition is satisfies if and only if $R_{p}^{N}=R_{u}(0) \in \mathfrak{C}\left(T_{p} N, W, b\right)$ and $\operatorname{Exp}(t \hat{A}(u)) \hat{A}(u) R_{p}^{N}=R_{u}^{\prime}(t) \in \mathfrak{C}\left(T_{p} N, W, b\right)_{L}$ for all $t \in J_{u}$. With the ideas of Lemma 1(b) (applied to $\beta_{v}^{\hat{A}(u)}$ with $v:=\hat{A}(u) R_{p}^{N}$ ) we find

$$
R_{u}^{\prime}\left(J_{u}\right) \in \mathfrak{C}\left(T_{p} N, W, b\right)_{L} \Longleftrightarrow \hat{A}(u)^{k} R_{p}^{N} \in \mathfrak{C}\left(T_{p} N, W, b\right)_{L} \text { for } k=1, \ldots, d
$$

Therefrom on the one hand we derive

$$
R_{u}\left(J_{u}\right) \subset \mathfrak{C}\left(T_{p} N, W, b\right) \Longrightarrow R_{u}(\mathbb{R}) \subset \mathfrak{C}\left(T_{p} N, W, b\right),
$$

and on the other hand we get that condition (41) is equivalent to

$$
R_{p}^{N} \in \mathfrak{C}\left(T_{p} N, W, b\right) \text { and } \hat{A}(u)^{k} R_{p}^{N} \in \mathfrak{C}\left(T_{p} N, W, b\right)_{L} \text { for all } u \in W \text { and } k=1, \ldots, d .
$$

The functions $P_{k}: u \mapsto \hat{A}(u)^{k} R_{p}^{N}$ are homogeneous polynomials $W \rightarrow \mathfrak{C}\left(T_{p} N\right)$ of degree $k$ corresponding to the symmetric $k$-linear maps

$$
L_{k}: W^{k} \rightarrow \mathfrak{C}\left(T_{p} N\right),\left(u_{1}, \ldots, u_{k}\right) \mapsto \frac{1}{k !} \sum\left(\hat{A}\left(u_{i_{1}}\right) \circ \cdots \circ \hat{A}\left(u_{i_{k}}\right)\right) R_{p}^{N},
$$

here the sum is taken over all permutations $\left(i_{1}, \ldots, i_{k}\right)$ of $\{1, \ldots, k\}$. Using the polarization formula (expressing $L_{k}\left(u_{1}, \ldots, u_{k}\right)$ as a sum of values of the polynomial $P_{k}$, see [Ca] p. 85) we get from (43):

Theorem 2 (II-parallel submanifolds in symmetric spaces). In the above situation there exists a II-parallel submanifold $M$ of the symmetric space $N$ with $p \in M, T_{p} M=W$ and $h_{p}^{M}=$ $b$ if and only if $R_{p}^{N} \in \mathfrak{C}\left(T_{p} N, W, b\right)$ and the maps $L_{k}(k=1, \ldots, d)$ take their values in $\mathfrak{C}\left(T_{p} N, W, b\right)_{L}$. If $\left(u_{1}, \ldots, u_{m}\right)$ is a basis of $W$, the latter condition is satisfied if and only if the finite number of values $L_{k}\left(u_{i_{1}}, \ldots, u_{i_{k}}\right)$ are contained in $\mathfrak{C}\left(T_{p} N, W, b\right)_{L}$.

Remember that because of Corollary 2 II-parallel submanifolds of a symmetric space again are locally symmetric.

\section{Semiparallelity of the second fundamental form and the euclidean Jordan triple property}

From Corollary 5 one sees that in a space $N$ of constant curvature there exists a II-parallel submanifold $M$ with the initial data $(p, W, b)$ if and only if $b$ is $R_{p}^{N}$-semiparallel. There is known a quite different sufficient and necessary tensorial condition for the existence of II-parallel submanifolds in standard spaces discovered by D. FERUs in [F] for $N=\mathbb{R}^{n}$ and later extended to the other spaces of constant curvature in [BR] (see Section 2 there), namely that a certain bilinear map $L: W \times W \rightarrow \operatorname{End}(W)$ associated with the triple $(p, W, b)$ is a euclidean Jordan triple system. Following Ferus' calculations I will prove (by purely tensorial calculations) now that in case of a constant curvature $N$ the conditions of Ferus and ours are equivalent. 
Let $V$ be a euclidean vector space, $R$ the curvature like tensor of a space of constant curvature $\varkappa$ on $V$ (i.e. $R(u, v)=\varkappa \cdot u \wedge v), W \in \mathrm{G}_{m}(V)$ and $b \in \mathrm{L}^{2}\left(W, W^{\perp}\right)$ a symmetric bilinear map. For this special case Equation (10) reads as

$$
R^{b}(u, v)=\varkappa \cdot u \wedge v-\left[A_{u}^{b}, A_{v}^{b}\right] \in \operatorname{End}_{-}(V) \text { for all } u, v \in W .
$$

Furthermore, by $S: W^{\perp} \rightarrow \operatorname{End}(W), \xi \mapsto S_{\xi}$ we denote the "shape operator" corresponding to $b$.

Lemma 3. (a) $\forall u, v, w, z \in W:\left\langle R^{b}(u, v) w, z\right\rangle=\left\langle R^{b}(w, z) u, v\right\rangle$.

(b) The orthogonal complement of the "first normal space" $N_{1}:=\operatorname{span}\{b(x, y) \mid x, y \in W\}$ is $N_{1}^{\perp}=\left\{\xi \in W^{\perp} \mid S_{\xi} \equiv 0\right\}$; and for every $\xi \in N_{1}^{\perp}$ and $u, v \in W$ we also have $S_{R^{b}(u, v) \xi}=0$.

Proof. The proof of (a) is trivial. For (b): The assertion $N_{1}^{\perp}=\left\{\xi \in W^{\perp} \mid S_{\xi} \equiv 0\right\}$ is easily proved. Suppose now $\xi=b(x, y)$ with $x, y \in W$ and calculate

$$
R^{b}(u, v) \xi=b\left(S_{\xi} u, v\right)-b\left(u, S_{\xi} v\right) \in N_{1} .
$$

Thus $R^{b}(u, v)$ leaves $N_{1}$ invariant, and consequently also $N_{1}^{\perp}$, because $R^{b}(u, v)$ is skew-adjoint. Thus we have also $S_{R^{b}(u, v) \xi}=0$.

Definition 5 (Semiparallelity of tensors). Let $T$ be a tensor of type $(k, r)$ on $V$. Then for all $u, v \in W$ we define the tensor $R^{b}(u, v) T$ of type $(k, r)$ by

$$
\left(R^{b}(u, v) T\right)\left(x_{1}, \ldots, x_{r}\right):= \begin{cases}-\sum T\left(x_{1}, \ldots, x_{i-1}, R^{b}(u, v) x_{i}, x_{i+1}, \ldots, x_{r}\right) & \text { for } k=0 \\ R^{b}(u, v)\left(T\left(x_{1}, \ldots, x_{r}\right)\right) & \\ -\sum T\left(x_{1}, \ldots, x_{i-1}, R^{b}(u, v) x_{i}, x_{i+1}, \ldots, x_{r}\right) & \text { for } k=1\end{cases}
$$

We apply the definition also to maps $T \in \operatorname{End}(W), T \in \mathrm{L}^{2}\left(W, W^{\perp}\right)$, the shape operator $W^{\perp} \times W \rightarrow W,(\xi, v) \mapsto S_{\xi} v$, etc. Furthermore, we say that any of these maps is semiparallel, if $R^{b}(u, v) T=0$ for all $u, v \in W$.

Example 8 (Semiparallel tensors). (a) The inner product of $V$ is semiparallel, because $R^{b}(u, v) \in$ End_ $_{-}(V)$; the curvature like tensor $R$ is semiparallel.

(b) The bilinear map $b$ is semiparallel if and only if the corresponding shape operator $S$ is semiparallel.

(c) If $b$ is semiparallel, then also $A^{b}: W \times V \rightarrow V,(u, v) \mapsto A_{u}^{b} v, \quad\left[A^{b}, A^{b}\right]: W^{2} \times V \rightarrow$ $V,\left(u_{1}, u_{2}, v\right) \mapsto\left[A_{u_{1}}^{b}, A_{u_{2}}^{b}\right] v$ and $W^{2} \times V \rightarrow V,\left(u_{1}, u_{2}, v\right) \mapsto R^{b}\left(u_{1}, u_{2}\right) v$ are semiparallel.

Now we introduce the following endomorphisms $W \rightarrow W$ for every $x, y \in W$ :

$$
S^{b}(x, y):=\varkappa\langle x, y\rangle \operatorname{id}_{W}+S_{b(x, y)} \quad \text { and } \quad L(x, y):=R^{b}(x, y)+S^{b}(x, y) .
$$

It can easily be proved that the following holds (see $[\mathrm{BR}]$ ):

$$
\forall x, y, z \in W: \quad\left(L(x, y) z=L(z, y) x \quad \text { and } \quad L(x, y)^{*}=L(y, x)\right) .
$$


Lemma 4. If $b$ is semiparallel, then also $S^{b}$ and $L$ are semiparallel; here $S^{b}$ and $L$ are considered as trilinear maps $W^{3} \rightarrow W$.

Proof. Use Example 8(c).

Definition 6 (Euclidean Jordan triple systems). The map $L$ is called a euclidean Jordan triple system, if besides (45) it satisfies

$$
\forall x, y, u, v \in W \quad: \quad[L(x, y), L(u, v)]=L(L(x, y) u, v)-L(u, L(y, x) v) .
$$

Observation 1. Equation (46) is equivalent to the two equations

$$
\begin{array}{ll}
\forall x, y, u, v \in W: & {\left[S^{b}(x, y), L(u, v)\right]=L\left(S^{b}(x, y) u, v\right)-L\left(u, S^{b}(x, y) v\right) \quad \text { and }} \\
\forall x, y, u, v \in W: & {\left[R^{b}(x, y), L(u, v)\right]=L\left(R^{b}(x, y) u, v\right)+L\left(u, R^{b}(x, y) v\right) .}
\end{array}
$$

Proof. Notice $L(x, y)+L(y, x)=2 S^{b}(x, y)$ and $L(x, y)-L(y, x)=2 R^{b}(x, y)$.

Observation 2. (48) exactly means that $L$ is semiparallel.

Observation 3. (47) is equivalent to

$$
\forall u, v \in W, \xi \in N_{1}: \quad\left[S_{\xi}, L(u, v)\right]=L\left(S_{\xi} u, v\right)-L\left(u, S_{\xi} v\right) .
$$

Proof. Use the definition of $S^{b}$ and take notice of the definition of $N_{1}$.

Observation 4. Equation (49) is equivalent to the two equations

$$
\begin{array}{ll}
\forall u, v \in W, \xi \in N_{1}: & {\left[S_{\xi}, S^{b}(u, v)\right]=R^{b}\left(S_{\xi} u, v\right)+R^{b}\left(S_{\xi} v, u\right) \quad \text { and }} \\
\forall u, v \in W, \xi \in N_{1}: & {\left[S_{\xi}, R^{b}(u, v)\right]=S^{b}\left(S_{\xi} u, v\right)-S^{b}\left(S_{\xi} v, u\right) .}
\end{array}
$$

Proof. Notice the second equation of $(45)$ and $L(u, v)+L(u, v)^{*}=2 S^{b}(u, v)$ and $L(u, v)-$ $L(u, v)^{*}=2 R^{b}(u, v)$.

Conclusion. $L$ is a euclidean Jordan triple system if and only if $L$ is semiparallel and the Equations (50) and (51) are satisfied.

Proof. Apply the Observations $1-4$.

Proposition 9. Equation (51) is satisfied if and only if $b$ is semiparallel.

Proof. Using the definitions of $S^{b}$ and Formula (44) we find that (51) is equivalent to $\forall u, v \in$ $W, \xi \in N_{1}:\left[S_{\xi}, R^{b}(u, v)\right]=S_{R^{b}(u, v) \xi}$, that means to

$$
\forall u, v, w \in W, \xi \in N_{1}: \quad\left(R^{b}(u, v) \cdot S\right)_{\xi} w=0,
$$


Thus we see, if $b$ is semiparallel, then because of Example 8(b) also $S$ is semiparallel and therefore (52), hence also (51) are satisfied. - Conversely, let (51) be satisfied. Because of Example $8(\mathrm{~b})$ it suffices to prove the semiparallelity of $S$; and because of (52) it remains to prove formula $\left(R^{b}(u, v) \cdot S\right)_{\xi} w=0$, i.e., $\left[S_{\xi}, R^{b}(u, v)\right]=S_{R^{b}(u, v) \xi}$ for all $u, v \in W$ and $\xi \in N_{1}^{\perp}$; but this previous fact is trivial because then $S_{\xi}$ and $S_{R^{b}(u, v) \xi}$ vanish according to Lemma 3(b).

Theorem 3 (A characterization of euclidean Jordan triple systems). L is a euclidean Jordan triple system if and only if $b$ is semiparallel.

Proof. For " $\Rightarrow$ ". If $L$ is a Jordan triple system, then (51) is satisfied according to the Conclusion and therefore $b$ is semiparallel by means of Proposition 9 .

For " $\Leftarrow$ ". We suppose the semiparallelity of $b$ and prove at first that $(50)$ is satisfied. For that let $u, v, w, z \in W$ and $\xi \in N_{1}$ be given and apply the right-hand side of (50) to $w$ and take the inner product with $z$; by means of Lemma 3(a) and the semiparallelity of $b$ we get

$$
\begin{aligned}
& \left\langle R^{b}\left(S_{\xi} u, v\right) w, z\right\rangle+\left\langle R^{b}\left(S_{\xi} v, u\right) w, z\right\rangle=\left\langle R^{b}(w, z) S_{\xi} u, v\right\rangle+\left\langle R^{b}(w, z) S_{\xi} v, u\right\rangle \\
& \quad=-\left\langle S_{\xi} u, R^{b}(w, z) v\right\rangle-\left\langle S_{\xi} v, R^{b}(w, z) u\right\rangle=-\left\langle\xi, b\left(u, R^{b}(w, z) v\right)\right\rangle-\left\langle\xi, b\left(v, R^{b}(w, z) u\right)\right\rangle \\
& \quad=-\left\langle\xi, R^{b}(w, z) b(u, v)\right\rangle=-\left\langle\xi,\left[A_{w}^{b}, A_{z}^{b}\right] b(u, v)\right\rangle=\left\langle\xi, b\left(z, S_{b(u, v)} w\right)-b\left(w, S_{b(u, v)} z\right)\right\rangle \\
& \quad=\left\langle S_{\xi} \circ S_{b(u, v)} w, z\right\rangle-\left\langle S_{\xi} \circ S_{b(u, v)} z, w\right\rangle=\left\langle\left[S_{\xi}, S_{b(u, v)}\right] w, z\right\rangle=\left\langle\left[S_{\xi}, S^{b}(u, v)\right] w, z\right\rangle .
\end{aligned}
$$

Thus (50) is valid. Furthermore, (51) is valid because of Proposition 9. Eventually $L$ is semiparallel because of Lemma 4 . Thus the Conclusion shows that $L$ is a Jordan triple system.

Corollary 6. $L$ is a euclidean Jordan triple system if and only if Equation (51) is satisfied.

Proof. Combine Proposition 9 and Theorem 3.

\section{Geometry over the Graßmann bundle of $N$}

Let $m$ be some integer with $1 \leq m \leq n-1$ and $\tau: \hat{N} \rightarrow N$ the corresponding Graßmann bundle over $N$; its fibre $\hat{N}_{q}$ over $q \in N$ is the Graßmann manifold $\mathrm{G}_{m}\left(T_{q} N\right)$ of $m$-dimensional linear subspaces of $T_{q} N$. Elements of $\hat{N}$ will be denoted by $W$.

In this section we will develop a general framework for the geometry of $m$-dimensional submanifolds of $N$ by introducing some fibre bundles over $\hat{N}$ together with connections. Example 9 at the end of this section will show how the usual submanifold geometry is embedded in the general apparatus.

The pull back of the tangent bundle $T N$

$$
\mathbb{E}:=\tau^{*} T N=\hat{N} \times_{N} T N
$$


is a vector bundle over $\hat{N}$; as its fibre $\mathbb{E}_{W}$ over $W$ is isomorphic to $T_{\tau(W)} N, \mathbb{E}$ inherits a metric from $T N$. By varying $W \in \hat{N}$ the orthogonal splittings $\mathbb{E}_{W}=W \oplus W^{\perp}$ give the orthogonal vector bundle splitting

$$
\mathbb{E}=\pi \oplus \Perp
$$

into the well known tautological bundle $\mathbb{\pi}$ and its orthogonal complement $\Perp$. For the sake of readability we will suppress superfluous letters $W$; so we write simply $\mathbb{E}_{W}=T_{\tau(W)} N$, $\pi_{W}=W, \mathrm{~L}(\mathbb{\pi}, \Perp)_{W}=\mathrm{L}\left(W, W^{\perp}\right)$, etc.

We will now introduce the principal fibre bundle of adapted orthonormal frames of $\mathbb{E}$. For that we fix a subspace $V_{0} \in \mathrm{G}_{m}\left(\mathbb{R}^{n}\right)$ and denote by $\mathbb{O}^{\top}$ resp. $\mathbb{O}^{\perp}$ the principal $\mathrm{O}\left(V_{0}\right)$ - resp. $\mathrm{O}\left(V_{0}^{\perp}\right)$-fibre bundle over $\hat{N}$, whose fibre over $W \in \hat{N}$ is the set $\mathbb{O}_{W}^{\top}$ resp. $\mathbb{O}_{W}^{\perp}$ of the linear isometries $V_{0} \rightarrow W$ resp. $V_{0}^{\perp} \rightarrow W^{\perp}$; we may interpret $\mathbb{O}^{\top}$ resp. $\mathbb{O}^{\perp}$ as the bundle of the orthonormal frames of $\pi$ resp. $\Perp .{ }^{6}$ Taking the fibre product of these bundles we obtain the principal fibre bundle

$$
\mathbb{P}:=\mathbb{O}^{\top} \times_{\hat{N}} \mathbb{O}^{\perp} \quad \text { with projektion } \quad \pi^{\mathbb{P}}: \mathbb{P} \rightarrow \hat{N}, u \mapsto u\left(V_{0}\right)
$$

(for the interpretation of $u$ see below) over $\hat{N}$ whose structure group is the isotropy group

$$
H:=\left\{g \in \mathrm{O}\left(\mathbb{R}^{n}\right) \mid g\left(V_{0}\right)=V_{0}\right\} \cong \mathrm{O}\left(V_{0}\right) \times \mathrm{O}\left(V_{0}^{\perp}\right)
$$

of the canonical action of $\mathrm{O}\left(\mathbb{R}^{n}\right)$ on the Graßmann manifold $\mathrm{G}_{m}\left(\mathbb{R}^{n}\right)$ at the "point" $V_{0}$. The elements of $\mathbb{P}_{W}$ are the linear isometries $u: \mathbb{R}^{n} \rightarrow \mathbb{E}_{W}=T_{\tau(W)} N$ with $u\left(V_{0}\right)=W$ and $u\left(V_{0}^{\perp}\right)=W^{\perp}$; the elements $u \mid V_{0} \in \mathbb{O}_{W}^{\top}$ resp. $u \mid V_{0}^{\perp} \in \mathbb{O}_{W}^{\perp}$ will be denoted by $u^{\top}$ resp. $u^{\perp}$. The vector bundles $\mathbb{E}, \mathbb{T}$ and $\Perp$ are associated with $\mathbb{P}$ via the maps

$$
\begin{aligned}
\mathbb{P} \times \mathbb{R}^{n} \rightarrow \mathbb{E},(u, x) \mapsto\left(\pi^{\mathbb{P}}(u), u(x)\right) \quad \text { resp. } \quad \mathbb{P} \times V_{0} \rightarrow \mathbb{\mathbb { N }},(u, x) \mapsto\left(\pi^{\mathbb{P}}(u), u^{\top}(x)\right) \\
\text { resp. } \quad \mathbb{P} \times V_{0}^{\perp} \rightarrow \mathbb{\Perp},(u, x) \mapsto\left(\pi^{\mathbb{P}}(u), u^{\perp}(x)\right) ;
\end{aligned}
$$

see $[\mathrm{Bo}]$ sect. 5.6.1. Now, we introduce also the associated vector bundles End $(\mathbb{E})$ and $\mathbb{B}:=$ $\mathrm{L}^{2}(\mathbb{T}, \mathbb{\Perp})$ with the typical fibres $\operatorname{End}_{-}\left(\mathbb{R}^{n}\right)$ and $\mathrm{L}^{2}\left(V_{0}, V_{0}^{\perp}\right)$; for instance, the latter bundle has the fibres $\mathbb{B}_{W}=\mathrm{L}^{2}\left(W, W^{\perp}\right)$ and is associated with $\mathbb{P}$ via the map

$$
\mathbb{P} \times \mathrm{L}^{2}\left(V_{0}, V_{0}^{\perp}\right) \rightarrow \mathbb{B},(u, b) \mapsto\left(\pi^{\mathbb{P}}(u), u^{\perp} \circ b \circ\left(u^{\top} \times u^{\top}\right)^{-1}\right) .
$$

Let $\sigma: \mathbb{E} \rightarrow \mathbb{E}$ be the bundle involution, which on the fibre $\mathbb{E}_{W}(W \in \hat{N})$ is the reflection in $W$. Thereby, we also can define an involution $\Sigma$ for the bundle End_( $\mathbb{E})$, namely by

$$
\Sigma_{W}(A):=\sigma_{W} \circ A \circ \sigma_{W}^{-1} \quad \text { for } \quad A \in \operatorname{End}_{-}\left(\mathbb{E}_{W}\right) .
$$

As obviously $\pi$ resp. $\Perp$ is the eigenbundle of $\sigma$ with respect to the eigenvalue 1 resp. -1 , the eigenspace splitting $\mathbb{H} \oplus \mathbb{M}$ of $\operatorname{End}_{-}(\mathbb{E})$ with respect to $\Sigma$ and the eigenvalues $1,-1$ is given by

$$
\begin{aligned}
\mathbb{H}_{W} & =\left\{A \in \text { End }_{-}\left(\mathbb{E}_{W}\right) \mid A(W) \subset W \text { and } A\left(W^{\perp}\right) \subset W^{\perp}\right\} \text { and } \\
\mathbb{M}_{W} & =\left\{A \in \text { End }_{-}\left(\mathbb{E}_{W}\right) \mid A(W) \subset W^{\perp} \text { and } A\left(W^{\perp}\right) \subset W\right\}
\end{aligned}
$$

\footnotetext{
${ }^{6}$ A natural choice for $V_{0}$ would be $V_{0}:=\mathbb{R}^{m} \times\{0\} \subset \mathbb{R}^{n}$; but with regard to the generalization to pseudoriemannian manifolds $N$ in Section 11 we have not specified $V_{0}$ in this way.
} 
(notice that the definition of $\mathbb{M}_{W}$ is in accordance with (7)) and for each $A \in$ End $_{-}\left(\mathbb{E}_{W}\right)$ the $\mathbb{H}_{W}$ - resp. $\mathbb{M}_{W}$-part is the endomorphism

$$
A^{\mathbb{H}}=\frac{1}{2}\left(A+\Sigma_{W}(A)\right) \quad \text { resp. } \quad A^{\mathbb{M}}=\frac{1}{2}\left(A-\Sigma_{W}(A)\right) .
$$

Of course, the vector bundles $\mathbb{H}$ and $\mathbb{M}$ also are canonically associated with $\mathbb{P}$. The typical fibre of $\mathbb{M}$ is

$$
\mathfrak{m}:=\mathbb{M}_{V_{0}}:=\left\{A \in \operatorname{End}_{-}\left(\mathbb{R}^{n}\right) \mid A\left(V_{0}\right) \subset V_{0}^{\perp} \text { and } A\left(V_{0}^{\perp}\right) \subset V_{0}\right\},
$$

on which $H$ acts by the isotropy representation $H \times \mathfrak{m} \rightarrow \mathfrak{m},(g, A) \mapsto g \circ A \circ g^{-1}$; the association of $\mathbb{M}$ with $\mathbb{P}$ is described by

$$
\rho^{\mathbb{M}}: \mathbb{P} \times \mathfrak{m} \rightarrow \mathbb{M},(u, A) \mapsto\left(\pi^{\mathbb{P}}(u), u \circ A \circ u^{-1}\right) .
$$

Remark 7. As already mentioned in Section 2 after Proposition $1, \mathrm{G}_{m}\left(\mathbb{R}^{n}\right)$ is a symmetric space $\cong \mathrm{O}\left(\mathbb{R}^{n}\right) / H$ with the isotropy group $H$ described in (53) and

$$
\theta:=\Theta_{V_{0}}: \mathfrak{m} \rightarrow T_{V_{0}} \mathrm{G}_{m}\left(\mathbb{R}^{n}\right),\left.A \mapsto \frac{\mathrm{d}}{\mathrm{d} t}\right|_{t=0}\left(\operatorname{Exp}(t A)\left(V_{0}\right)\right)
$$

is an isomorphism from the subspace $\mathfrak{m} \subset$ End_ $_{-}\left(\mathbb{R}^{n}\right)$ described in (57) onto the tangent space $T_{V_{0}} \mathrm{G}_{m}\left(\mathbb{R}^{n}\right)$.

Of course one can carry out the same construction for each fibre $\hat{N}_{q}=\mathrm{G}_{m}\left(T_{q} N\right)$ of the Graßmann bundle $\tau$ with respect to any fixed $W \in \hat{N}_{q}$ (instead of $V_{0} \in \mathrm{G}_{m}\left(\mathbb{R}^{n}\right)$ ). Then the decomposition of the Lie algebra End $\left(T_{q} N\right)=$ End $_{-}\left(\mathbb{E}_{W}\right)$ of the group $\mathrm{O}\left(T_{q} N\right)$ (replacing the decomposition $\left.\mathfrak{o}\left(\mathbb{R}^{n}\right)=\mathfrak{h} \oplus \mathfrak{m}\right)$ is exactly the splitting End ${ }_{-}\left(\mathbb{E}_{W}\right)=\mathbb{H}_{W} \oplus \mathbb{M}_{W}$ and instead of $\theta$ we have the isomorphism

$$
\Theta_{W}: \mathbb{M}_{W} \rightarrow T_{W} \mathrm{G}_{m}\left(T_{q} N\right)=T_{W} \hat{N}_{q},\left.A \mapsto \frac{\dot{\mathrm{d}}}{\mathrm{d} t}\right|_{t=0}(\operatorname{Exp}(t A)(W))
$$

already described in (8).

We will also use that for every subspace $W \in \hat{N}$ there exist canonical Lie algebra homomorphisms

$$
\mathbb{H}_{W} \rightarrow \operatorname{End}\left(\mathbb{B}_{W}\right), A \mapsto A_{\mathbb{B}} \quad \text { and } \quad \mathbb{H}_{W} \rightarrow \operatorname{End}\left(\mathbb{M}_{W}\right), A \mapsto A_{\mathbb{M}}
$$

which for every $A \in \mathbb{H}_{W}, b \in \mathbb{B}_{W}$ and $C \in \mathbb{M}_{W}$ are described by

$$
\begin{aligned}
& \left(A_{\mathbb{B}} b\right)\left(v_{1}, v_{2}\right)=A\left(b\left(v_{1}, v_{2}\right)\right)-b\left(A v_{1}, v_{2}\right)-b\left(v_{1}, A v_{2}\right) \quad \text { and } \\
& \left.\left(A_{\mathbb{M}} C\right)(v)=A(C(v))-C(A v)\right)=[A, C](v) .
\end{aligned}
$$

Remark 8 (A better understanding of semiparallelity). If $W \in \hat{N}_{q}$ is curvature invariant, then for every $b \in \mathbb{B}_{W}$ the values of the "tensor" $R^{b}$ associated with the quadruple $\left(T_{q} N, W, b, R_{q}^{N}\right)$ (according to (10)) lie in $\mathbb{H}_{W}$; using the terminology of (61) we therefore can state: $b$ is $R_{q}^{N}$-semiparallel if and only if

$$
R^{b}\left(u_{1}, u_{2}\right)_{\mathbb{B}} b=0 \quad \text { for all } \quad u_{1}, u_{2} \in W .
$$


As the sections of the vector bundle $\mathbb{E}$ can be identified with the vector fields of $N$ along the projection $\tau: \hat{N} \rightarrow N$ and as for every vector field $\hat{X} \in \Gamma(T \hat{N})$ and section $Z \in \Gamma(\mathbb{E})$ the covariant derivative $\nabla^{N} \hat{X} Z$ can again be considered as a section of $\mathbb{E}$ (see footnote 1), we can define metric covariant derivatives $\nabla^{\top}$ resp. $\nabla^{\perp}$ on $\pi$ resp. $\Perp$ via the splitting $\mathbb{E}=\mathbb{\pi} \oplus \Perp$ : For $\hat{v} \in T_{W} \hat{N}, Y \in \Gamma(\mathbb{\pi})$ and $\zeta \in \Gamma(\Perp)$ they are given by

$$
\nabla_{\hat{v}}^{\top} Y:=\left(W \text {-part of } \nabla^{N}{ }_{\hat{v}} Y\right) \in \pi_{W} \quad \text { and } \quad \nabla_{\hat{v}}^{\perp} \zeta:=\left(W^{\perp} \text {-part of } \nabla^{N} \hat{v} \zeta\right) \in \Perp_{W} .
$$

Furthermore, these covariant derivatives correspond to linear connections on the principal fibre bundles $\mathbb{O}^{\top}$ and $\mathbb{O}^{\perp}$; in a further step the latter connections produce a linear connection $\mathcal{H}^{\mathbb{P}}$ on the product bundle $\mathbb{P}$. It can be characterized by the following fact:

For every curve $\xi: J \rightarrow \hat{N}$, parameter $t_{0} \in J$ and element $u \in \mathbb{P}_{\xi\left(t_{0}\right)}$ the $\mathcal{H}^{\mathbb{P}}$ horizontal lift of $\xi$ through $u$ is the curve $u_{\xi}: J \rightarrow \mathbb{P}$, which is determined by $u_{\xi}\left(t_{0}\right)=u$ and the fact that the functions

$$
t \mapsto u_{\xi}(t)\left(x^{\top}\right) \in \xi(t) \text { for } x^{\top} \in V_{0} \quad \text { and } \quad t \mapsto u_{\xi}(t)\left(x^{\perp}\right) \in \xi(t)^{\perp} \text { for } x^{\perp} \in V_{0}^{\perp}
$$

are parallel sections of $\left(\pi, \nabla^{\top}\right)$ and $\left(\Perp, \nabla^{\perp}\right)$ along $\xi$, respectively;

again we have identified sections of $\mathbb{T}$ and $\Perp$ with vector fields of $N$ along $\tau \circ \xi$; so we will do in future.

As is well known the connection $\mathcal{H}^{\mathbb{P}}$ induces connections on every fibre bundle associated with $\mathbb{P}$ in a canonical way (see [KN] Vol. I, p. 87) and in case of associated vector bundles this connections correspond to covariant derivatives. For the vector bundles $\pi$ and $\Perp$ we come back to $\nabla^{\top}$ and $\nabla^{\perp}$. For $\mathbb{E}$ the "new" covariant derivative $\nabla^{\mathbb{E}}$ is different from that given by $\nabla^{N}$; loosely speaking, it is the "sum" of $\nabla^{\top}$ and $\nabla^{\perp}$; that means, $\mathbb{T}$ and $\Perp$ are parallel subbundles of $\left(\mathbb{E}, \nabla^{\mathbb{E}}\right) ;^{7}$ thus, for all $\hat{v} \in T_{W} \hat{N}, Y \in \Gamma(\mathbb{\pi})$ and $\zeta \in \Gamma(\mathbb{\Perp})$ we have

$$
\nabla_{\hat{v}}^{\top} Y=\nabla_{\hat{v}}^{\mathbb{E}} Y \text { and } \quad \nabla_{\hat{v}}^{\frac{1}{}} \zeta=\nabla_{\hat{v}}^{\mathbb{E}} \zeta
$$

and for all $\hat{u}_{1}, \hat{u}_{2} \in T_{W} \hat{N}$

$$
\begin{aligned}
& R^{\pi}\left(\hat{u}_{1}, \hat{u}_{2}\right)=R^{\mathbb{E}}\left(\hat{u}_{1}, \hat{u}_{2}\right) \mid W \quad \text { and } \quad R^{\Perp}\left(\hat{u}_{1}, \hat{u}_{2}\right)=R^{\mathbb{E}}\left(\hat{u}_{1}, \hat{u}_{2}\right) \mid W^{\perp}, \\
& \text { hence } R^{\mathbb{E}}\left(\hat{u}_{1}, \hat{u}_{2}\right) \in \mathbb{H}_{M} .
\end{aligned}
$$

Following the general procedure (described in [KN] Vol. I, p. 88) we obtain: If $\xi: J \rightarrow \hat{N}$ and $u_{\xi}: J \rightarrow \mathbb{P}$ are as in (64), then

$$
\left.\varphi(t):=u_{\xi}(t) \circ u_{\xi}\left(t_{0}\right)^{-1}: \mathbb{E}_{\xi\left(t_{0}\right)} \rightarrow \mathbb{E}_{\xi(t)} \quad \text { is the parallel displacement } \quad \stackrel{t}{\|_{t_{0}}} \xi\right)^{\mathbb{E}}
$$

in $\left(\mathbb{E}, \nabla^{\mathbb{E}}\right)$ along the curve $\xi$; it satisfies $\varphi(t)\left(\xi\left(t_{0}\right)\right)=\xi(t)$ and $\varphi(t)\left(\xi\left(t_{0}\right)^{\perp}\right)=\xi(t)^{\perp} .8$

Moreover, for every $b \in \mathbb{B}_{\xi\left(t_{0}\right)}$ and $A \in \mathbb{M}_{\xi\left(t_{0}\right)}$ the functions $b_{\xi}: J \rightarrow \mathbb{B}$ and $A_{\xi}: J \rightarrow \mathbb{M}$ determined by

$$
b_{\xi}(t) \circ(\varphi(t) \times \varphi(t)) \mid\left(\xi\left(t_{0}\right) \times \xi\left(t_{0}\right)\right)=\varphi(t) \circ b \quad \text { and } \quad A_{\xi}(t) \circ \varphi(t)=\varphi(t) \circ A
$$

\footnotetext{
${ }^{7} \mathrm{~A}$ general reason will be described in Remark 10.

${ }^{8} \mathrm{An}$ inhabitant of $\xi\left(t_{0}\right)$ moved with this subspace will consider $t \mapsto \varphi(t)$ as the natural parallel transport of the tangent space $T_{\tau \circ \xi\left(t_{0}\right)} N$.
} 
are the parallel sections in $\mathbb{B}$ resp. $\mathbb{M}$ along $\xi$ with $b_{\xi}\left(t_{0}\right)=b$ and $A_{\xi}\left(t_{0}\right)=A$. This fact implies

$$
\begin{aligned}
& \left(\nabla_{\hat{X}}^{\mathbb{B}} h\right)\left(Y_{1}, Y_{2}\right)=\nabla_{\hat{X}}^{\perp}\left(h\left(Y_{1}, Y_{2}\right)\right)-h\left(\nabla_{\hat{X}}^{\top} Y_{1}, Y_{2}\right)-h\left(Y_{1}, \nabla_{\hat{X}}^{\top} Y_{2}\right), \\
& \left(\nabla_{\hat{X}}^{\mathbb{M}} S\right) Z=\nabla_{\hat{X}}^{\mathbb{E}} S(Z)-S\left(\nabla_{\hat{X}}^{\mathbb{E}} Z\right), \quad \text { and therefore } \\
& \left(\nabla^{\mathbb{M}}{ }_{\hat{X}} S\right) Y=\nabla_{\hat{X}}^{\perp} S(Y)-S\left(\nabla_{\hat{X}}^{\top} Y\right) \quad \text { and } \quad\left(\nabla_{\hat{X}}^{\mathbb{M}} S\right) \zeta=\nabla_{\hat{X}}^{\top} S(\zeta)-S\left(\nabla_{\hat{X}}^{\perp} \zeta\right)
\end{aligned}
$$

for every $\hat{X} \in \Gamma(T \hat{N}), h \in \Gamma(\mathbb{B}), S \in \Gamma(\mathbb{M}), Z \in \Gamma(\mathbb{E}), Y, Y_{1}, Y_{2} \in \Gamma(\mathbb{\pi})$ and $\zeta \in \Gamma(\Perp \mathbb{})$. Using the terminology of (61) and (62) and taking in account (65) and (66) we find that the curvature tensors of the connections $\nabla^{\mathbb{B}}, \nabla^{\mathbb{M}}$ and $\nabla^{\mathbb{E}}$ are related by

$$
\begin{aligned}
& \forall \hat{u}_{1}, \hat{u}_{2} \in T_{W} \hat{N}: \quad R^{\mathbb{B}}\left(\hat{u}_{1}, \hat{u}_{2}\right)=R^{\mathbb{E}}\left(\hat{u}_{1}, \hat{u}_{2}\right)_{\mathbb{B}} \quad \text { and } \\
& R^{\mathbb{M}}\left(\hat{u}_{1}, \hat{u}_{2}\right)=R^{\mathbb{E}}\left(\hat{u}_{1}, \hat{u}_{2}\right)_{\mathbb{M}} .
\end{aligned}
$$

An essential tool in the following will be the difference tensor $\hat{\nu}$ of $\nabla^{N}$ and $\nabla^{\mathbb{E}}$, which we consider as a differential 1-form, whose values lie in the vector bundle $\mathbb{M}$, as is seen from (76):

$$
\forall \hat{v} \in T_{W} \hat{N}, Z \in \Gamma(\mathbb{E}): \quad \hat{\nu}(\hat{v}) Z_{q}:=\nabla^{N} \hat{v} Z-\nabla_{\hat{v}}^{\mathbb{E}} Z .
$$

Because of (63) and (65) for $Y \in \Gamma(\mathbb{\pi})$ resp. $\zeta \in \Gamma(\Perp)$ we have

$$
\nabla_{\hat{v}}^{N} Y=\nabla_{\hat{v}}^{\top} Y+\hat{\nu}(\hat{v}) Y_{W} \quad \text { and } \quad \nabla_{\hat{v}}^{N} \zeta=\hat{\nu}(\hat{v}) \zeta_{W}+\nabla_{\hat{v}}^{\perp} \zeta
$$

with

$$
\hat{\nu}(\hat{v}) Y_{W} \in W^{\perp} \quad \text { and } \quad \hat{\nu}(\hat{v}) \zeta_{W} \in W
$$

Comparing this with Example 2(a) we may interpret $\hat{\nu}$ as a universal second fundamental form and the Equations (75) as a universal Gauß equation resp. a universal Weingarten equation, see also Example 9. Eventually, let us call in mind the Cartan derivative of the 1 -form $\hat{\nu}$; it is characterized by

$$
\mathrm{d} \hat{\nu}\left(\hat{X}_{1}, \hat{X}_{2}\right)=\nabla_{\hat{X}_{1}}^{\mathbb{M}}\left(\hat{\nu}\left(\hat{X}_{2}\right)\right)-\nabla_{\hat{X}_{2}}^{\mathbb{M}}\left(\hat{\nu}\left(\hat{X}_{1}\right)\right)-\hat{\nu}\left(\left[\hat{X}_{1}, \hat{X}_{2}\right]\right) .
$$

Using (74), (70) and Cartan's structure equation for the curvature tensor we get the following relation between the curvature tensors of the connections $\nabla^{N}$ and $\nabla^{\mathbb{E}}$

$$
\forall \hat{u}_{1}, \hat{u}_{2} \in T_{W} \hat{N}: \quad R^{N}\left(\tau_{*} \hat{u}_{1}, \tau_{*} \hat{u}_{2}\right)=R^{\mathbb{E}}\left(\hat{u}_{1}, \hat{u}_{2}\right)+\left[\hat{\nu}\left(\hat{u}_{1}\right), \hat{\nu}\left(\hat{u}_{2}\right)\right]+\mathrm{d} \hat{\nu}\left(\hat{u}_{1}, \hat{u}_{2}\right) ;
$$

the $\mathbb{H}_{W}$ - resp. $\mathbb{M}_{W}$-part of the endomorphism $R^{N}\left(\tau_{*} \hat{u}_{1}, \tau_{*} \hat{u}_{2}\right) \in \operatorname{End}_{-}\left(\mathbb{E}_{W}\right)$ is $R^{\mathbb{E}}\left(\hat{u}_{1}, \hat{u}_{2}\right)+$ $\left[\hat{\nu}\left(\hat{u}_{1}\right), \hat{\nu}\left(\hat{u}_{2}\right)\right]$ resp. d $\hat{\nu}\left(\hat{u}_{1}, \hat{u}_{2}\right)$; see also the following example.

Example 9 (Embedding of submanifold geometry into the preceding framework). Let $M$ be an $m$-dimensional submanifold of $N$. Then its Gauß map $\xi:=\xi^{M}: M \rightarrow \hat{N}, p \mapsto T_{p} M$ is an injective immersion (because $\tau \circ \xi$ is the inclusion $M \hookrightarrow N$ ) and its second fundamental form $h:=h^{M}$ a section in $\mathbb{B}$ along $\xi$. In particular, the image $\hat{M}:=\xi(M)$ is a submanifold of $\hat{N}$. The pull back bundles $\xi^{*} \mathbb{E}, \xi^{*} \mathbb{T}$ and $\xi^{*} \Perp$ are canonical isomorphic to $\left.T N\right|_{M}, T M$ and $\perp M$, respectively; and $\xi^{*} \mathbb{P}=M \times_{\hat{N}} \mathbb{P}$ is the principal bundle of $M$-adapted frames over $M$, see $[\mathrm{KN}]$ Vol.2,p. 1. For all vectors $v, u, u_{1}, u_{2} \in T_{p} M$ and $z \in \perp_{p} M$ and all sections $Y \in \Gamma(T M)$ and $\zeta \in \Gamma(\perp M)$ the Formulas (63), (65) and (75) yield

$$
\begin{aligned}
& \nabla_{v}^{\mathbb{E}} Y=\nabla^{M}{ }_{v} Y \text { and } \quad \nabla^{\mathbb{E}} \zeta=\nabla^{\perp M}{ }_{v} \zeta, \\
& \hat{\nu}\left(\xi_{*} u\right) v=h_{p}(u, v) \quad \text { and } \quad \hat{\nu}\left(\xi_{*} u\right) z=-S_{z}^{M} u, \quad \text { hence } \quad \hat{\nu}\left(\xi_{*} u\right)=A_{u}^{h_{p}}
\end{aligned}
$$


(see Definition 1(a) and notice that $Y$ and $\zeta$ can be considered as sections in $\mathbb{E}$ along the map $\xi)$; and therefore

$$
R^{\mathbb{E}}\left(\xi_{*} u_{1}, \xi_{*} u_{2}\right) \mid T_{p} M=R^{M}\left(u_{1}, u_{2}\right) \quad \text { and } \quad R^{\mathbb{E}}\left(\xi_{*} u_{1}, \xi_{*} u_{2}\right) \mid \perp_{p} M=R^{\perp M}\left(u_{1}, u_{2}\right) .
$$

The first two formulas of (79) show also that the isometries $\varphi(t)$ of the $M$-split-parallel displacement of $T_{p} N$ along a curve $c: J \rightarrow M$ with $p:=c(0)$ (see Definition 4) coincide with the isometries, which in (67) are associated with the curve $\xi \circ c: J \rightarrow \hat{N}$. Furthermore, from (2), (69), (72) and (80) we obtain

$$
\begin{aligned}
& \nabla_{v}^{\mathbb{B}} h=\bar{\nabla}_{v} h \text { and }\left(R^{\mathbb{B}}\left(\xi_{*} u_{1}, \xi_{*} u_{2}\right) h_{p}\right)\left(v_{1}, v_{2}\right)= \\
& =R^{\perp M}\left(u_{1}, u_{2}\right)\left(h_{p}\left(v_{1}, v_{2}\right)\right)-h_{p}\left(R^{M}\left(u_{1}, u_{2}\right) v_{1}, v_{2}\right)-h_{p}\left(v_{1}, R^{M}\left(u_{1}, u_{2}\right) v_{2}\right) .
\end{aligned}
$$

In particular, $M$ is a II-parallel submanifold if and only if $h^{M}$ is a parallel section in the bundle $\left(\mathbb{B}, \nabla^{\mathbb{B}}\right)$. Eventually, Formula $(78)$ can be seen as a combination of the curvature equations of Gauß , Coddazi and Ricci by means of Example 2 and the following expressions, which are crucial in the two versions of the Codazzi equation

$$
\begin{aligned}
\mathrm{d} \hat{\nu}\left(\xi_{*} u_{1}, \xi_{*} u_{2}\right) v & =\left(\bar{\nabla}_{u_{1}} h\right)\left(u_{2}, v\right)-\left(\bar{\nabla}_{u_{2}} h\right)\left(u_{1}, v\right) \quad \text { and } \\
\mathrm{d} \hat{\nu}\left(\xi_{*} u_{1}, \xi_{*} u_{2}\right) z & =\left(\nabla_{u_{2}} S^{M}\right)_{z} u_{1}-\left(\nabla_{u_{1}} S^{M}\right)_{z} u_{2} ;
\end{aligned}
$$

for the calculation use (2), (70), (77) and (79). If the tangent space $T_{p} M$ is curvature invariant, then we know from the Codazzi equation that the right-hand sides of the previous two formulas vanish, and we obtain from (10), (78) and (79) that the tensor $R^{b}$ associated with the quadruple $\left(T_{p} N, T_{p} M, h_{p}, R_{p}^{N}\right)$ satisfies $R^{b}\left(u_{1}, u_{2}\right)=R^{\mathbb{E}}\left(\xi_{*} u_{1}, \xi_{*} u_{2}\right)$. See also Remark 15 .

Lemma 5 (Parallel displacement in $\mathbb{E}$ along distinguished curves). Let a curve $c: J \rightarrow N$ with $0 \in J$ and elements $W \in \hat{N}_{c(0)}$ and $A \in \mathbb{M}_{W}$ be given. Then the isometries

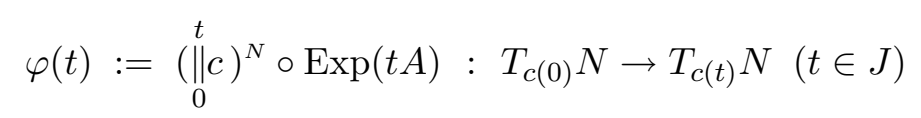

describe the parallel displacement in $\left(\mathbb{E}, \nabla^{\mathbb{E}}\right)$ along the curve $\xi: J \rightarrow \hat{N}, t \mapsto \varphi(t)(W)$ (see also (67)); obviously $c=\tau \circ \xi$ holds. Furthermore, if denotes, then we have

$$
A_{\xi}: J \rightarrow \mathbb{M}, t \mapsto \hat{\nu}(\dot{\xi}(t))
$$

is the parallel section in $\mathbb{M}$ along $\xi$ with $A_{\xi}(0)=A$.

Proof. For every $v \in T_{p} N$ let us introduce the vector field $Z_{v}: t \mapsto \varphi(t) v$ along $c$. Since by definition $\hat{Z}_{v}: t \mapsto \operatorname{Exp}(t A) v$ is the backward parallel displacement of $Z_{v}$ and $\hat{Z}_{v}^{\prime}=$ $\hat{Z}_{A v}$, Proposition $2\left(\right.$ a) proves $\nabla_{\partial}^{N} Z_{v}=Z_{A v}$. Now, we start with an element $v \in W$, then by construction we have $Z_{v}(t) \in \xi(t)=\pi_{\xi(t)}$ and $\left(\nabla_{\partial}^{N} Z_{v}\right)(t)=Z_{A v}(t) \in \xi(t)^{\perp}=\Perp_{\xi(t)}$ because $A v \in W^{\perp}$. By means of (63) and (65) we obtain therefore $\left(\nabla_{\partial}^{\mathbb{E}} Z_{v}\right)(t)=\left(\nabla_{\partial}^{\top} Z_{v}\right)(t)=$ $\left(\xi(t)\right.$-part of $\left.\left(\nabla_{\partial}^{N} Z_{v}\right)(t)\right)=0$. Thereby we have proved that $Z_{v}$ is a parallel section in $\mathbb{E}$ along $\xi$. For $v \in W^{\perp}$ the previous result is proved analogously. Consequently, all sections $Z_{v}$ with $v \in \mathbb{E}_{W}$ are $\nabla^{\mathbb{E}}$-parallel, and therefore $\varphi(t)$ is the parallel displacement in $\mathbb{E}$ as stated in the proposition. We call in mind that this displacement already appeared in (67). Finally, in order to prove (82) we calculate for every $v \in T_{q} N$ by means of $(74): \hat{\nu}(\dot{\xi}(t))(\varphi(t) v)=$ $\hat{\nu}(\dot{\xi}(t)) Z_{v}(t)=\left(\nabla_{\partial}^{N} Z_{v}\right)(t)-\left(\nabla_{\partial}^{\mathbb{E}} Z_{v}\right)(t)=Z_{A v}(t)=\varphi(t)(A v) ;$ in particular, we have $\hat{\nu}(\dot{\xi}(0))(v)=$ $\hat{\nu}(\dot{\xi}(0))(\varphi(0) v)=\varphi(0)(A v)=A v$, hence $\hat{\nu}(\dot{\xi}(0))=A$; moreover, according to (68) the section $t \mapsto \hat{\nu}(\dot{\xi}(t))$ is $\nabla^{\mathbb{M}}$-parallel. 
Remark 9 . In the situation of Lemma 5 the function $A_{\xi}$ is not only a parallel section in $\mathbb{M}$, but it satisfies even

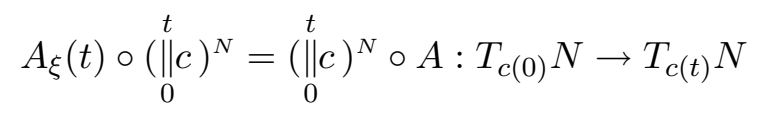

because of (68) and $\operatorname{Exp}(t A) \circ A=A \circ \operatorname{Exp}(t A)$.

Let us end this section with a further remark.

Remark 10. If two fibre bundles $\mathbb{F}$ and $\tilde{\mathbb{F}}$ with typical fibres $F$ resp. $\tilde{F}$ are associated with $\mathbb{P}$ via maps $\rho: \mathbb{P} \times F \rightarrow \mathbb{F}$ resp. $\tilde{\rho}: \mathbb{P} \times \tilde{F} \rightarrow \tilde{\mathbb{F}}$ and if $\phi: F \rightarrow \tilde{F}$ is an $H$-equivariant map, then there exists a unique fibre morphism $\Phi: \mathbb{F} \rightarrow \tilde{\mathbb{F}}$ satisfying $\Phi(\rho(u, f))=\tilde{\rho}(u, \phi(f))$; see [Bo] sect. 6.5.5. Fibre morphisms which occur in this way shall be called $\mathbb{P}$-morphisms in future. Such a $\mathbb{P}$-morphism is compatible with the connections induced on the bundles by $\mathcal{H}^{\mathbb{P}}$, that means: if $\alpha: J \rightarrow \mathbb{F}$ is a horizontal curve, then also $\Phi \circ \alpha: J \rightarrow \tilde{\mathbb{F}}$ is horizontal. If $\mathbb{F}$ and $\tilde{\mathbb{F}}$ are associated vector bundles and $\phi$ is linear, then $\Phi$ is a vetor bundle morphism satisfying

$$
\nabla_{\hat{X}}^{\tilde{\mathbb{F}}}(\Phi \circ s)=\Phi \circ\left(\nabla_{\hat{X}}^{\mathbb{F}} s\right) \text { for all } \hat{X} \in \Gamma(T \hat{N}) \text { and } s \in \Gamma(\mathbb{F}) ;
$$

consequently the curvature tensors of the connections $\nabla^{\mathbb{F}}$ and $\nabla^{\tilde{\mathbb{F}}}$ are related by

$$
\forall \hat{u}_{1}, \hat{u}_{2} \in T_{W} \hat{N}, z \in \mathbb{F}_{W}: \quad R^{\tilde{\mathbb{F}}}\left(\hat{u}_{1}, \hat{u}_{2}\right) \Phi(z)=\Phi\left(R^{\mathbb{F}}\left(\hat{u}_{1}, \hat{u}_{2}\right) z\right) .
$$

For instance, since $\pi$ and $\Perp$ are associated subbundles of $\mathbb{E}$, these subbundles are parallel in $\mathbb{E}$ as was already noticed above. Further important applications will follow in the next sections.

\section{Geometry of the manifold $\hat{N}$}

In this section we introduce a linear connection $\hat{\nabla}$ (with torsion) on $\hat{N}$, which for our purpose is adapted to the geometry of the Graßmann bundle in the best possible way. For instance, the maps $\xi_{u}: t \mapsto W_{t u} \in \hat{N}$, where $W_{t u}$ is defined as before Theorem 1, will become geodesics of $\hat{N}$.

If we consider $\hat{N}$ as a fibre bundle associated with the bundle $\mathrm{L}(N)$ of all linear frames of $N$ via the map

$$
\hat{\rho}: \mathrm{L}(N) \times \mathrm{G}_{m}\left(\mathbb{R}^{n}\right) \rightarrow \hat{N},(u, V) \mapsto u(V),
$$

then the linear connection of $\mathrm{L}(N)$ corresponding to $\nabla^{N}$ induces a connection $\hat{\mathcal{H}}$ on the bundle $\tau: \hat{N} \rightarrow N$, i.e., we obtain a splitting

$$
T \hat{N}=\hat{\mathcal{H}} \oplus \hat{\mathcal{V}} \text { with } \hat{\mathcal{V}}:=\operatorname{Ker}\left(\tau_{*}\right) \text { (the latter means } \hat{\mathcal{V}}_{W}=T_{W} \hat{N}_{q} \text { for every } W \in \hat{N}_{q} \text { ) }
$$

into the horizontal and vertical subbundle (see $[\mathrm{KN}]$ Vol.1, p. 87); more explicitely, a curve $\xi: J \rightarrow \hat{N}$ is horizontal if and only if for any $t_{0}, t \in J$ we have

$$
\xi(t)=W_{c}(t):=\left(\|_{t_{0}}^{t} c\right)^{N}(W) \quad \text { with } c:=\tau \circ \xi \text { and } W:=\xi\left(t_{0}\right) \quad(\text { see }(1)) .
$$

Let

$$
\eta: T \hat{N} \rightarrow \hat{\mathcal{H}} \quad \text { and } \quad \nu: T \hat{N} \rightarrow \hat{\mathcal{V}}
$$

denote the projections corresponding to the splitting $T \hat{N}=\hat{\mathcal{H}} \oplus \hat{\mathcal{V}}$. 
Theorem 4 (The isomorphism $\boldsymbol{T} \hat{\boldsymbol{N}} \rightarrow \mathbb{E} \oplus \mathbb{M}$ ). The isomorphisms $\Theta_{W}$ from Remark 7 are the "fibres" of a vector bundle $\mathbb{P}$-isomorphism $\Theta: \mathbb{M} \rightarrow \hat{\mathcal{V}}$, and the following identity holds

$$
\Theta \circ \hat{\nu}=\nu
$$

If additionally we define the vector bundle morphism $\hat{\eta}: T \hat{N} \rightarrow \mathbb{E}, T_{W} \hat{N} \ni \hat{v} \mapsto\left(W, \tau_{*} \hat{v}\right)=$ $\left(W, \tau_{*} \circ \eta(\hat{v})\right)$, then

$$
(\hat{\eta}, \hat{\nu}): T \hat{N} \rightarrow \mathbb{E} \oplus \mathbb{M}
$$

becomes a fibre bundle isomorphism. Via this map the splitting $T \hat{N}=\hat{\mathcal{H}} \oplus \hat{\mathcal{V}}$ corresponds with the direct sum structure of $\mathbb{E} \oplus \mathbb{M}$.

Proof. As is well known, also $\hat{\mathcal{V}}$ is a fibre bundle over $N$ associated with $\mathrm{L}(N)$, namely via the map $\rho^{\hat{\mathcal{V}}}: \mathrm{L}(N) \times T \mathrm{G}_{m}\left(\mathbb{R}^{n}\right) \rightarrow \hat{\mathcal{V}},(u, w) \mapsto \hat{\rho}_{u *} w$ with $\hat{\rho}_{u}:=\hat{\rho}(u, \cdot): \mathrm{G}_{m}\left(\mathbb{R}^{n}\right) \rightarrow \hat{N}$. As $\mathbb{P} \times T_{V_{0}} \mathrm{G}_{m}\left(\mathbb{R}^{n}\right)$ is a submanifold of $\mathrm{L}(N) \times T \mathrm{G}_{m}\left(\mathbb{R}^{n}\right)$, we can define the restriction $\tilde{\rho}:=$ $\rho^{\hat{\nu}} \mid\left(\mathbb{P} \times T_{V_{0}} \mathrm{G}_{m}\left(\mathbb{R}^{n}\right)\right)$. Now it turns out that $\hat{\mathcal{V}}$ is a vector bundle over $\hat{N}$ associated with the principal bundle $\mathbb{P}$ via this map.

Furthermore, the isomorphism $\theta: \mathfrak{m} \rightarrow T_{V_{0}} G_{m}\left(\mathbb{R}^{n}\right)$ from (59) is $H$-equivariant. As $\mathfrak{m}$ is the typical fibre of the associated bundle $\mathbb{M}$, we therefore can apply Remark 10 and obtain a $\mathbb{P}_{-}$ isomorphism $\Theta: \mathbb{M} \rightarrow \hat{\mathcal{V}}$. If for $W \in \hat{N}, u \in \mathbb{P}_{W}$ and $A \in \mathbb{M}_{W}$ we put $\tilde{A}:=u^{-1} \circ A \circ u \in \mathfrak{m}$, then we get by means of $(60)$

$$
\begin{aligned}
& \Theta(A)=\tilde{\rho}(u, \theta(\tilde{A}))=\left.\hat{\rho}_{u *} \frac{\dot{\mathrm{d}}}{\mathrm{d} t}\right|_{t=0}\left(\operatorname{Exp}(t \tilde{A})\left(V_{0}\right)\right)=\left.\frac{\dot{\mathrm{d}}}{\mathrm{d} t}\right|_{t=0} \hat{\rho}_{u} \circ\left(\operatorname{Exp}(t \tilde{A})\left(V_{0}\right)\right) \\
& =\left.\frac{\dot{\mathrm{d}}}{\mathrm{d} t}\right|_{t=0} u\left(\operatorname{Exp}(t \tilde{A})\left(V_{0}\right)\right)=\left.\frac{\dot{\mathrm{d}}}{\mathrm{d} t}\right|_{t=0}\left(\operatorname{Exp}(t A)\left(u\left(V_{0}\right)\right)\right)=\left.\frac{\dot{\mathrm{d}}}{\mathrm{d} t}\right|_{t=0}(\operatorname{Exp}(t A)(W))=\Theta_{W}(A) .
\end{aligned}
$$

Proof of $\Theta \circ \hat{\nu}(\hat{v})=\nu(\hat{v})$ for $\hat{v} \in T_{W} \hat{N}$ and $W \in \hat{N}_{q}$ : According to the splitting $T_{W} \hat{N}=$ $\hat{\mathcal{H}}_{W} \oplus \hat{\mathcal{V}}_{W}$ we may restrict the proof to the two cases $\hat{v} \in \hat{\mathcal{H}}_{W}$ and $\hat{v} \in \hat{\mathcal{V}}_{W}$. In both cases we will choose an appropriate curve $c: J \rightarrow N$ with $0 \in J$ and $c(0)=q$ and an element $A \in \mathbb{M}_{W}$ such that $\hat{v}=\dot{\xi}(0)$, where $\xi: J \rightarrow \hat{N}$ is the curve defined in Lemma 5 ; because of (82), we know then $\hat{\nu}(\hat{v})=A$ and therefore $\Theta \circ \hat{\nu}(\hat{v})=\Theta(A)$; thus we have to prove $\Theta(A)=\nu(\hat{v})$ in the two cases.

Case $\hat{v} \in \hat{\mathcal{H}}_{W}$. Let $c$ be some curve with $\dot{c}(0)=\tau_{*} \hat{v}$ and $A=0 \in \mathbb{M}_{W}$. Then the curve $\xi$ from Lemma 5 is exactly the horizontal lift $W_{c}$ described in $(86), \dot{\xi}(0)=\hat{v}$ holds and we have $\Theta(A)=0=\nu(\hat{v})$.

Case $\hat{v} \in \hat{\mathcal{V}}_{W}=T_{W} \hat{N}_{q}$. Now we put $c \equiv q$. Because of Remark 7 there exists exactly one $A \in \mathbb{M}_{W}$ with $\Theta_{W}(A)=\hat{v}$. Since we have $\xi(t)=\operatorname{Exp}(t A)(W)$ now, we get $\dot{\xi}(0)=\Theta_{W}(A)=\hat{v}$. In this situation $\Theta(A)=\hat{v}=\nu(\hat{v})$ is satisfied trivially.

Thus the equation $\Theta \circ \hat{\nu}=\nu$ is proved completely. Therefrom we will now derive that $(\hat{\eta}, \hat{\nu})$ is a fibre bundle isomorphism: As $\operatorname{dim}\left(\mathbb{E}_{W} \oplus \mathbb{M}_{W}\right)=\operatorname{dim} N+\operatorname{dim} \hat{N}_{q}=\operatorname{dim} \hat{\mathcal{H}}_{W}+\operatorname{dim} \hat{\mathcal{V}}_{W}=$ $\operatorname{dim} T_{W} \hat{N}$, it is sufficient to show the injectivity of $(\hat{\eta}, \hat{\nu})_{W}$. For that we choose some $\hat{v} \in T_{W} \hat{N}$ with $\hat{\eta}(\hat{v})=\hat{\nu}(\hat{v})=0$; then we get $\nu(\hat{v})=0$ and therefore $\hat{v} \in \hat{\mathcal{H}}_{W}$; consequently, $\tau_{*} \hat{v}=$ $\hat{\eta}(\hat{v})=0$ implies $\hat{v}=0$.

Remark 11. At every point $W \in \hat{N}_{q}$ the 1 -form $\hat{\nu}$ is characterized by 
(i) $\hat{\nu}$ vanishes on the horizontal subspace $\hat{\mathcal{H}}_{W}$ and

(ii) for vectors $\hat{v}$ of the vertical subspace $\mathcal{V}_{W}$ we have $\hat{\nu}(\hat{v}) \mid W=d x(\hat{v})$, where $x$ : $\mathrm{G}_{m}\left(T_{q} N\right) \supset \mathcal{U}(W) \rightarrow \mathrm{L}\left(W, W^{\perp}\right)$ is the canonical chart of $\mathrm{G}_{m}\left(T_{q} N\right)$ about $W$ described in (4).

Proof. Since according to Theorem $4 \Theta \circ \hat{\nu} \mid T_{W} \hat{N}$ is the projection $T_{W} \hat{N} \rightarrow \hat{\mathcal{V}}_{W}$ along $\hat{\mathcal{H}}_{W}$, assertion (i) is obtained immediately, and assertion (ii) follows with (9) by the following calculation: $d x(\hat{v})=d x\left(\Theta_{W}(\hat{\nu}(\hat{v}))\right)=\hat{\nu}(\hat{v}) \mid W$.

This characterization of $\hat{\nu}$ may remind the reader to the connection map of a vector bundle $E \rightarrow$ $N$ with a linear connection $\nabla^{E}$; if $s: J \rightarrow E$ is a section in $E$ along a curve $c: J \rightarrow N$, then the connection map $K: T E \rightarrow E$ describes the relation between the two possible derivatives of $s$, namely by $K \dot{s}=\nabla_{\partial}^{E} s$. This connection map vanishes on the horizontal subbundle of $T E$, and on each vertical subspace $T_{e} E_{p}$ it coincides with the canonical identification of $T_{e} E_{p}$ with the linear space $E_{p}$.

In order to emphasize that $\hat{\nu}$ is a natural extension of the second fundamental forms of all $m$ dimensional submanifolds $M \subset N$ we show how Proposition 1 can be deduced from the above characterization of $\hat{\nu}$. For this purpose we need the following

Lemma 6. Let $\tau: B \rightarrow N$ be a fibre bundle associated with a principal fibre bundle $\pi: P \rightarrow N$, on which a connection $\mathcal{H}$ (invariant with respect to the structure group of $\pi$ ) is given. Then for every curve $\alpha: J \rightarrow B$ with $0 \in J$ the following is true:

$$
\left.\frac{\dot{\mathrm{d}}}{\mathrm{d} t}\right|_{t=0}\left(\left(\|_{t}^{0} c\right)^{B} \alpha(t)\right)=\text { vertical part of } \dot{\alpha}(0)
$$

here $c$ denotes the curve $\tau \circ \alpha$ and $\underset{t}{\left(\|_{t}\right)^{B}}$ the horizontal backward displacement $B_{c(t)} \rightarrow B_{c(0)}$ in $B$ along $c$ induced by the connection $\mathcal{H}$ (see [KN] Vol.1,p.87).

Proof. Let $F$ denote the typical fibre of the bundle $\tau$ and $\rho: P \times F \rightarrow B$ the map, which associates $\tau$ with $\pi$. Furthermore, let $u: J \rightarrow P$ denote a horizontal lift of $c$. Then for every $t \in J$ we have

$$
\stackrel{0}{(\| c)^{B}}=\rho_{u(0)} \circ \rho_{u(t)}^{-1} \quad \text { with } \quad \rho_{u(t)}:=\rho(u(t), \bullet) .
$$

Now, there exists exactly one $\mathrm{C}^{\infty}$-curve $\beta: J \rightarrow F$ such that $\alpha(t)=\rho(u(t), \beta(t))$ for all $t \in J$. Hence we have

$$
\dot{\alpha}(0)=\left(\rho_{u(0)}\right)_{*} \dot{\beta}(0)+\left(\rho^{\beta(0)}\right)_{*} \dot{u}(0) .
$$

By definition of the horizontal structure of the bundle $\tau$ the vector $\left(\rho^{\beta(0)}\right)_{*} \dot{u}(0)$ is horizontal. Therefore, $\left(\rho_{u(0)}\right)_{*} \dot{\beta}(0)$ is the vertical part of $\dot{\alpha}(0)$, which is of our present interest. Hence, the following calculation finishes our proof:

$$
\left(\rho_{u(0)}\right)_{*} \dot{\beta}(0)=\left.\left.\frac{\dot{\mathrm{d}}}{\mathrm{d} t}\right|_{t=0}\left(\rho_{u(0)} \circ \beta\right)(t) \underset{(88)}{=} \frac{\dot{\mathrm{d}}}{\mathrm{d} t}\right|_{t=0}\left(\left(\|_{t}^{0}\right)^{B}\left(\rho_{u(t)} \circ \beta(t)\right)\right)=\left.\frac{\dot{\mathrm{d}}}{\mathrm{d} t}\right|_{t=0}\left((\underset{t}{0})^{B} \alpha(t)\right) .
$$


Deduction of Proposition 1 from Remark 11. Let $\xi^{M}: M \rightarrow \hat{N}$ denote the Gauß map of the submanifold $M \subset N$. From Example 9 we know

$$
\hat{\nu}\left(\xi_{*}^{M} u\right) \mid W=h_{p}(u, \cdot) \quad \text { with } \quad W=T_{p} M \quad \text { for all } u \in T_{p} M .
$$

If in the situation of Proposition 1 we put $\alpha:=\xi^{M} \circ c$, then we get $\xi_{*}^{M} u=\dot{\alpha}(0)$ and therefore because of Remark 11:

$$
h_{p}(u, \cdot)=\hat{\nu}\left(\xi_{*}^{M} u\right) \mid W=\hat{\nu}(\text { vertical part of } \dot{\alpha}(0)) \mid W=d x(\text { vertical part of } \dot{\alpha}(0)) ;
$$

The previous lemma shows that the vertical part of $\dot{\alpha}(0)$ is $\left.\frac{\dot{\mathrm{d}}}{\mathrm{d} t}\right|_{t=0}\left(\left(\|_{t}^{0}\right)^{B} \alpha(t)\right)$. Therefore, we obtain (with the notations of Proposition 1)

$$
h_{p}(u, \cdot)=d x\left(\left.\frac{\mathrm{d}}{\mathrm{d} t}\right|_{t=0}\left((\| c)_{t}^{B}\left(T_{c(t)} M\right)\right)\right)=\left.\frac{\mathrm{d}}{\mathrm{d} t}\right|_{t=0}(\underbrace{\left(x\left((\| c)^{B}\left(T_{c(t)} M\right)\right)\right.}_{=\ell(t)})=\ell^{\prime}(t) .
$$

Construction of the connection $\hat{\nabla}$. Because of the isomorphy $T \hat{N} \cong \mathbb{E} \oplus \mathbb{M}$ the tangent bundle $T \hat{N}$ can be considered as being associated with $\mathbb{P}$, too, namely with typical fibre $\mathbb{R}^{n} \oplus \mathfrak{m}$ and via the map

$$
\rho^{T \hat{N}}: \mathbb{P} \times\left(\mathbb{R}^{n} \oplus \mathfrak{m}\right) \rightarrow T \hat{N}
$$

characterized by

$$
\tau_{*} \rho^{T \hat{N}}(u, x, A)=u(x) \quad \text { and } \quad \hat{\nu}\left(\rho^{T \hat{N}}(u, x, A)\right)=\left(\pi^{\mathbb{P}}(u), u \circ A \circ u^{-1}\right) ;
$$

see (57) and (58). Therefore, the manifold $\hat{N}$ bears a linear connection $\hat{\nabla}$ which comes from the connection $\mathcal{H}^{\mathbb{P}}$. It is characterized by the following fact (see $[\mathrm{KN}]$ Vol. 1, p. 87):

For every curve $\xi: J \rightarrow \hat{N}$, parameter $t_{0} \in J$ and vector $\hat{v} \in T_{\xi\left(t_{0}\right)} \hat{N}$ the $\hat{\nabla}-$ parallel vector field $\hat{v}_{\xi}: J \rightarrow T \hat{N}$ along $\xi$ with $\hat{v}_{\xi}\left(t_{0}\right)=\hat{v}$ can be described by means of a basis $u \in \mathbb{P}_{\xi\left(t_{0}\right)}$ and the horizontal lift $u_{\xi}: J \rightarrow \mathbb{P}$ of $\xi$ with $u_{\xi}\left(t_{0}\right)=u$ :

$$
\hat{v}_{\xi}: t \mapsto \rho^{T \hat{N}}\left(u_{\xi}(t), x, A\right) \quad \text { with } \quad(x, A):=\left(\rho_{u}^{T \hat{N}}\right)^{-1}(\hat{v}) .
$$

Proposition 10 (Properties of $\hat{\nabla}$ ). The covariant derivative $\hat{\nabla}$ on $\hat{N}$ induced by the connection $\mathcal{H}^{\mathbb{P}}$ is characterized by the following formulas valid for all $\hat{v} \in T_{W} \hat{N}$ and $\hat{Y} \in \Gamma(T \hat{N})$

$$
\tau_{*} \hat{\nabla}_{\hat{v}} \hat{Y}=\nabla^{\mathbb{E}} \tau_{*} \hat{Y}=\nabla^{N} \tau_{*} \hat{Y}-\hat{\nu}(\hat{v})\left(\tau_{*} \hat{Y}_{W}\right) \quad \text { and } \quad \hat{\nu}\left(\hat{\nabla}_{\hat{v}} \hat{Y}\right)=\nabla_{\hat{v}}^{\mathbb{M}} \hat{\nu}(\hat{Y}) .
$$

The torsion and curvature tensors of $\hat{\nabla}$ are given by the following formulas: If we put $u_{i}:=\tau_{*} \hat{u}_{i}$ for $\hat{u}_{i} \in T_{W} \hat{N}$, then:

$$
\begin{aligned}
& \tau_{*} T^{\hat{N}}\left(\hat{u}_{1}, \hat{u}_{2}\right)=\hat{\nu}\left(\hat{u}_{2}\right) u_{1}-\hat{\nu}\left(\hat{u}_{1}\right) u_{2} \quad \text { and } \quad \hat{\nu}\left(T^{\hat{N}}\left(\hat{u}_{1}, \hat{u}_{2}\right)\right)=\mathbb{M}_{W} \text {-part of } R^{N}\left(u_{1}, u_{2}\right), \\
& \begin{aligned}
\tau_{*} R^{\hat{N}}\left(\hat{u}_{1}, \hat{u}_{2}\right) \hat{u}_{3} & =R^{\mathbb{E}}\left(\hat{u}_{1}, \hat{u}_{2}\right) u_{3} \\
& =\left(\left(\mathbb{H}_{W} \text {-part of } R^{N}\left(u_{1}, u_{2}\right)\right)-\left[\hat{\nu}\left(\hat{u}_{1}\right), \hat{\nu}\left(\hat{u}_{2}\right)\right]\right) u_{3}
\end{aligned} \\
& \begin{aligned}
\hat{\nu}\left(R^{\hat{N}}\left(\hat{u}_{1}, \hat{u}_{2}\right) \hat{u}_{3}\right) & =R^{\mathbb{M}}\left(\hat{u}_{1}, \hat{u}_{2}\right) \hat{\nu}\left(\hat{u}_{3}\right)=\left[R^{\mathbb{E}}\left(\hat{u}_{1}, \hat{u}_{2}\right), \hat{\nu}\left(\hat{u}_{3}\right)\right] .
\end{aligned}
\end{aligned}
$$

Because of (93) the torsion $T^{\hat{N}}$ does not vanish identically. 
Proof. For $(92)$ we use that $(\hat{\eta}, \hat{\nu})$ is a $\mathbb{P}$-morphism and Formula (74). Formula (93) follows by means of Cartan's structure equation for the torsion, because the torsion of $N$ vanishes, and by means of (77) and (78); for (94) we use that $\hat{\eta}$ and $\hat{\nu}$ are $\mathbb{P}$-morphisms and the Formulas (84), (78), (73) and (62). Finally we get $T^{\hat{N}}\left(\hat{u}_{1}, \hat{u}_{2}\right)=\hat{\nu}\left(\hat{u}_{2}\right) u_{1} \neq 0$ for $\hat{u}_{1} \in \hat{\mathcal{H}}_{W} \backslash\{0\}$ and $\hat{u}_{2} \in \hat{\mathcal{V}}_{W} \backslash\{0\}$ by means of (93).

Remark 12 (Special parallel subbundles of $\boldsymbol{T} \hat{\boldsymbol{N}}$ ). For every $W \in \hat{N}$ and $u \in \mathbb{P}_{W}$ the following holds:

$\rho_{u}^{T \hat{N}}(\{0\} \times \mathfrak{m})=\hat{\mathcal{V}}_{W}, \rho_{u}^{T \hat{N}}\left(\mathbb{R}^{n} \times\{0\}\right)=\hat{\mathcal{H}}_{W}$ and $\rho_{u}^{T \hat{N}}\left(V_{0} \times\{0\}\right)=\left\{\hat{v} \in \hat{\mathcal{H}}_{W} \mid \tau_{*} \hat{v} \in W\right\}=: \mathcal{D}_{W}$.

Because of $(91) \hat{\mathcal{V}}, \hat{\mathcal{H}}, \mathcal{D}$ and $\mathcal{D}^{\perp}:=\rho^{T \hat{N}}\left(\mathbb{P} \times V_{0}^{\perp} \times\{0\}\right)$ therefore are $\hat{\nabla}$-parallel subbundles of $T \hat{N}$.

Proposition 11 (Geodesics in $\hat{\mathbf{N}})$. Let an arbitrary vector $\hat{u} \in T_{W} \hat{N}\left(W \in \hat{N}_{q}\right)$ be given, put $A:=\hat{\nu}(\hat{u})$ and $u:=\tau_{*} \hat{u}$, and denote the maximal helical arc in $N$ associated with the pair $(u, A)$ by $c: J \rightarrow N$ (see Definition 3). Then the curve

$$
\xi: J \rightarrow \hat{N}, t \mapsto \underset{0}{\stackrel{t}{\| c})^{N}}(\operatorname{Exp}(t A)(W))
$$

is the maximal geodesic of $(\hat{N}, \hat{\nabla})$ with $\dot{\xi}(0)=\hat{u}$.

Proof. Notice that $\xi$ is the curve which is associated with $(c, A)$ by Lemma 5. Since $c=\tau \circ \xi$, we get in particular, $\tau_{*} \dot{\xi}(0)=\dot{c}(0)=u=\tau_{*} \hat{u}$; on the other hand (82) shows $\hat{\nu}(\dot{\xi}(0))=A=\hat{\nu}(\hat{u})$; thus we obtain $\dot{\xi}(0)=\hat{u}$ by Theorem 4 . In order to prove that $\xi$ is a geodesic, define the isometries $\varphi(t):=\underset{0}{t} \| c)^{N} \circ \operatorname{Exp}(t A)$ and the parallel section $Z_{u}: t \mapsto \varphi(t) u$ of $\mathbb{E}$ along $\xi$, compare Lemma 5. Then we use (92) and Definition 3 to calculate

$$
\tau_{*}\left(\hat{\nabla}_{\partial} \dot{\xi}\right)(t)=\nabla_{\partial}^{\mathbb{E}} \dot{c}(t)=\nabla_{\partial}^{\mathbb{E}} Z_{u}(t)=0 ;
$$

on the other hand, (92), (82) and (68) induce

$$
\hat{\nu}\left(\hat{\nabla}_{\partial} \dot{\xi}\right)=\nabla_{\partial}^{\mathrm{M}} \hat{\nu}(\dot{\xi})=\nabla_{\partial}^{\mathrm{M}} A_{\xi}=0 .
$$

Thus we have proved $\hat{\nabla}_{\partial} \dot{\xi}=0$, i.e. $\xi$ is a geodesic with the initial velocity $\hat{u}$. It is maximally extended; indeed, if $\xi$ would be extendable to some larger interval $J^{*} \supseteqq J$, then $\tau \circ \xi$ would extend $c$ to $J^{*}$ in contradiction to Proposition 2 (d).

Corollary 7. If $N$ is complete, then $\hat{N}$ is geodesically complete.

Proof. See Remark 1(b).

Now we will compare $\hat{\nabla}$ with the Levi-Civita connection $\nabla^{L C}$ of the riemannian metric usually considered on the Graßmann bundle. This metric can be defined as follows:

On the vector space $\mathfrak{m}$ we choose the inner product

$$
\left\langle\left\langle A_{1}, A_{2}\right\rangle\right\rangle:=-\operatorname{trace}\left(A_{2} \circ A_{1}\right),
$$


which is invariant with respect to the isotropy representation $\mathrm{Ad}: H \times \mathfrak{m} \rightarrow \mathfrak{m},(g, A) \mapsto g \circ A \circ g^{-1}$ (see (57) and (59)). Then, on the one hand, there exists a unique riemannian metric on $\mathrm{G}_{m}\left(\mathbb{R}^{n}\right)$, with respect to which the maps

$$
\psi_{g}: \mathrm{G}_{m}\left(\mathbb{R}^{n}\right) \rightarrow \mathrm{G}_{m}\left(\mathbb{R}^{n}\right), V \mapsto g(V) \quad \text { for } \quad g \in \mathrm{O}\left(\mathbb{R}^{n}\right)
$$

are isometries and which satisfies

$$
\left\langle\theta\left(A_{1}\right), \theta\left(A_{2}\right)\right\rangle_{V_{0}}=\left\langle\left\langle A_{1}, A_{2}\right\rangle\right\rangle
$$

I will call it the canonical riemannian metric of the Graßmann manifold $G_{m}\left(\mathbb{R}^{n}\right)$, and I will use this notation also for the Grassmannian $\mathrm{G}_{m}(V)$ of other euclidean vector spaces $V$. - On the other hand, as the isotropy group $H$ acts on the euclidean space $\mathbb{R}^{n} \times \mathfrak{m}$ by the isometries $g \times \operatorname{Ad}(g)(g \in H)$, there exists a unique riemannian metric on $\hat{N}$ (which from now on will be referred to as "the" riemannian metric of $\hat{N}$ ) such that for every $u \in \mathbb{P}_{W}$ the map

$$
\rho_{u}^{T \hat{N}}: \mathbb{R}^{n} \oplus \mathfrak{m} \rightarrow T_{W} \hat{N} \quad \text { is a linear isometry ; }
$$

here $\rho^{T \hat{N}}$ is the map, by which $T \hat{N}$ was associated with $\mathbb{P}$ (see (89)) and which was also used in the construction of the connection $\hat{\nabla}$. In distinction to the latter connection the Levi-Civita connection of the riemannian manifold $\hat{N}$ will be denoted by $\nabla^{L C}$.

Proposition 12 (On the riemannian manifold $\hat{N}) .^{9} \quad$ (a) The riemannian metric of $\hat{N}$ has the following properties: ${ }^{10}$

(i) $\hat{\mathcal{V}} \perp \hat{\mathcal{H}}$

(ii) $\tau: \hat{N} \rightarrow N$ is a riemannian submersion.

(iii) The riemannian metric of $\hat{N}$ induces on each fibre $\hat{N}_{q}=\mathrm{G}_{m}\left(T_{q} N\right)$ the canonical riemannian metric of this Grassmannian, and for every orthonormal basis $u \in \mathrm{L}_{q}(N)$ the map $\hat{\rho}_{u}: \mathrm{G}_{m}\left(\mathbb{R}^{n}\right) \rightarrow \hat{N}$ is an isometry onto the fibre $\hat{N}_{q}$ (for the definition of $\hat{\rho}$ see (85)).

(b) For every curve $c: J \rightarrow N$ and all parameters $t_{1}, t_{2} \in J$ the horizontal displacement

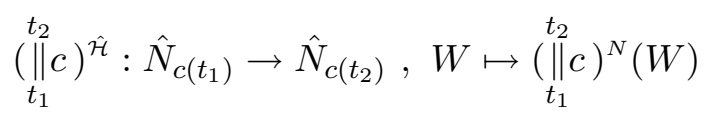

in $\hat{N}$ along c (described by Formula (86)) is an isometry, if the fibres $\hat{N}_{c(t)}$ are considered as riemannian submanifolds of the riemannian manifold $\hat{N}$. Hence the holonomy group of the connection $\hat{\mathcal{H}}$ with respect to a point $q \in N$ is a subgroup of the isometry group of $\hat{N}_{q}$.

(c) If $\hat{\Omega}: T \hat{N} \times \hat{N} T \hat{N} \rightarrow \hat{\mathcal{V}}$ denotes the "curvature tensor" of $\hat{\mathcal{H}}$ characterized by

$$
\forall \hat{X}, \hat{Y} \in \Gamma(T \hat{N}): \quad \hat{\Omega}(\hat{X}, \hat{Y})=-\nu([\eta \hat{X}, \eta \hat{Y}]) \quad(\text { for } \eta, \nu \text { see }(87))
$$

\footnotetext{
${ }^{9}$ This proposition is based on an unpublished paper of H. Reckziegel (Cologne).

${ }^{10}$ These properties show that the riemannian metric of the bundle space $\hat{N}$ coincides with the one which J. VILMS constructed in [V1] in some more general situations (see also [Be] Th.9.55 on p. 249).
} 
then the following O'Neill equations are true:

$$
\begin{array}{lll}
\forall \hat{X}, \hat{Y} \in \Gamma(\hat{\mathcal{H}}): & \nu\left(\nabla_{\hat{X}}^{L C} \hat{Y}\right)=-\frac{1}{2} \cdot \hat{\Omega}(\hat{X}, \hat{Y}) \quad \text { and } \\
\forall \hat{X}, \hat{Y} \in \Gamma(\hat{\mathcal{H}}): & \tau_{*} \nabla_{\hat{X}}^{L C} \hat{Y}=\nabla^{N} \tau_{*} \hat{Y} .
\end{array}
$$

Formula (98) says that $-\frac{1}{2} \cdot \hat{\Omega} \mid\left(\hat{\mathcal{H}} \times{ }_{\hat{N}} \hat{\mathcal{H}}\right)$ can be considered as second fundamental form of the subbundle $\hat{\mathcal{H}} \subset T \hat{N}$.

(d) The shape operator ${ }^{11} S^{\hat{\mathcal{H}}}: \hat{\mathcal{V}} \times_{\hat{N}} \hat{\mathcal{H}} \rightarrow \hat{\mathcal{H}}$ of the subbundle $\hat{\mathcal{H}}$ given by

$$
\forall \hat{U} \in \Gamma(\hat{\mathcal{V}}), \hat{X} \in \Gamma(\hat{\mathcal{H}}): \quad S_{\hat{U}}^{\hat{\mathcal{H}}} \hat{X}=-\eta\left(\nabla_{\hat{X}}^{L C} \hat{U}\right)
$$

is related to the curvature tensor $\hat{\Omega}$ by

$$
\forall \hat{U} \in \Gamma(\hat{\mathcal{V}}), \hat{X}, \hat{Y} \in \Gamma(\hat{\mathcal{H}}):\left\langle S_{\hat{U}}^{\hat{\mathcal{H}}} \hat{X}, \hat{Y}\right\rangle=-\frac{1}{2} \cdot\langle\hat{\Omega}(\hat{X}, \hat{Y}), \hat{U}\rangle .
$$

Besides Formula (99) we have

$$
\forall \hat{U} \in \Gamma(\hat{\mathcal{V}}), \hat{X} \in \Gamma(\hat{\mathcal{H}}): \quad \tau_{*} \nabla_{\hat{U}}^{L C} \hat{X}=\nabla^{N} \tau_{*} \hat{X}-\tau_{*} S_{\hat{U}}^{\hat{\mathcal{H}}} \hat{X} .
$$

(e) The vector bundle $\hat{\mathcal{V}}$ is a $\nabla^{L C}$-autoparallel subbundle of $T \hat{N}$, and therefore the fibres $\hat{N}_{q}$ are totally geodesic submanifolds of the riemannian manifold $\hat{N}$. Therefrom one derives for all $\hat{X} \in \Gamma(\hat{\mathcal{H}})$ and $\hat{U}, \hat{U}_{1}, \hat{U}_{2} \in \Gamma(\hat{\mathcal{V}})$ :

$$
\begin{aligned}
\nu\left(\nabla_{\hat{X}}^{L C} \hat{U}\right) & =\nu[\hat{X}, \hat{U}] \quad \text { and thus } \\
\hat{X}\left\langle\hat{U}_{1}, \hat{U}_{2}\right\rangle & =\left\langle\left[\hat{X}, \hat{U}_{1}\right], \hat{U}_{2}\right\rangle+\left\langle\hat{U}_{1},\left[\hat{X}, \hat{U}_{2}\right]\right\rangle .
\end{aligned}
$$

(f) In addition to Equation (101) the following is true:

$$
\forall \hat{U} \in \Gamma(\hat{\mathcal{V}}), \hat{X} \in \Gamma(\hat{\mathcal{H}}): \nabla_{\hat{U}}^{L C} \hat{X} \in \Gamma(\hat{\mathcal{H}}) .
$$

Proof. For (a). (i) follows immediately from Remark 12. Since we also get $\left\|\tau_{*}\left(\rho_{u}^{T \hat{N}}(x, 0)\right)\right\|=$ $\|u(x)\|=\|x\|=\|(x, 0)\|=\left\|\rho_{u}^{T \hat{N}}(x, 0)\right\|$, we have verified (ii). In order to verify (iii) we first prove the following relation between the maps $\hat{\rho}_{u}$ and $\rho_{u}^{T \hat{N}}$ :

$$
\forall(u, A) \in \mathbb{P}_{W} \times \mathfrak{m}: \rho_{u}^{T \hat{N}}(0, A)=\Theta_{W}\left(u \circ A \circ u^{-1}\right)=\hat{\rho}_{u *}(\theta(A)) .
$$

For (105). Since $u\left(V_{0}\right)=W$ holds and $\rho_{u}^{T \hat{N}}(0, A)$ is vertical, Formula (90) shows $\rho_{u}^{T \hat{N}}(0, A)=$ $\Theta_{W}\left(u \circ A \circ u^{-1}\right)=\left.\frac{\dot{\mathrm{d}}}{\mathrm{d} t}\right|_{t=0}\left(\operatorname{Exp}\left(t \cdot u \circ A \circ u^{-1}\right)(W)\right)=\left.\frac{\dot{\mathrm{d}}}{\mathrm{d} t}\right|_{t=0}\left(\hat{\rho}_{u}\left(\operatorname{Exp}(t \cdot A)\left(V_{0}\right)\right)\right)=\hat{\rho}_{u *}(\theta(A))$.

Now let a point $V \in \mathrm{G}_{m}\left(\mathbb{R}^{n}\right)$ and a vector $v \in T_{V} \mathrm{G}_{m}\left(\mathbb{R}^{n}\right)$ be given. Then there exist a transformation $g \in \mathrm{O}\left(\mathbb{R}^{n}\right)$ and an element $A \in \mathfrak{m}$ such that

$$
g\left(V_{0}\right)=\psi_{g}\left(V_{0}\right)=V, \quad \text { hence } u \circ g \in \mathbb{P}_{u(V)}, \quad \text { and } \quad \psi_{g *} \theta(A)=v \quad(\text { see }(95)) .
$$

Because of $\hat{\rho}_{u \circ g}=\hat{\rho}_{u} \circ \psi_{g}$ we obtain by means of (106) and (105)

$$
\hat{\rho}_{u *} v=\hat{\rho}_{u *} \circ \psi_{g *}(\theta(A))=\hat{\rho}_{u \circ g *}(\theta(A))=\rho_{u \circ g}^{T \hat{N}}(0, A),
$$

\footnotetext{
${ }^{11}$ I use the notations second fundamental form and shape operator of $\hat{\mathcal{H}}$ because of the statements (c) and (d); but one must notice that this second fundamental form is skew-symmetric and therefore this shape operators $S_{\hat{u}}^{\hat{\mathcal{H}}}$ are skew-adjoint.
} 
hence

$$
\left\|\hat{\rho}_{u *} v\right\|=\left\|\rho_{u \circ g}^{T \hat{N}}(0, A)\right\|=\|A\| \underset{(96)}{=}\|\theta(A)\| \underset{(95)}{=}\left\|\psi_{g *} \theta(A)\right\| \underset{(106)}{=}\|v\|
$$

Thus $\hat{\rho}_{u}$ is an isometric embedding into $\hat{N}$ with image $\hat{N}_{q}$. Next we prove that the induced riemannian metric on $\hat{N}_{q}=\mathrm{G}_{m}\left(T_{q} N\right)$ is the canonical one. We continue with the preceding notations. If $\tilde{A}_{1}, \tilde{A}_{2} \in \mathbb{M}_{W}$ are given, we put $A_{k}:=u^{-1} \circ \tilde{A}_{k} \circ u \in \mathfrak{m}$. Since $\hat{\rho}_{u}$ is isometric, we obtain

$$
\begin{aligned}
\left\langle\left\langle\tilde{A}_{1}, \tilde{A}_{2}\right\rangle\right\rangle & =-\operatorname{trace}\left(\tilde{A}_{2} \circ \tilde{A}_{1}\right)=-\operatorname{trace}\left(A_{2} \circ A_{1}\right)=\left\langle\left\langle A_{1}, A_{2}\right\rangle\right\rangle \\
& \underset{(96)}{=} \\
\underset{(105)}{=} & \left\langle\Theta\left(A_{1}\right), \theta\left(A_{2}\right)\right\rangle_{\mathrm{G}_{m}\left(\mathbb{R}^{n}\right), V_{0}}=\left\langle\hat{\rho}_{1 *}\right), \Theta\left(\tilde{A}_{2}\right\rangle_{\hat{N}_{q}, W} .
\end{aligned}
$$

Thus $\langle\cdot, \cdot\rangle_{\hat{N}_{q}}$ satisfies the analogous Formula (96) for the Grassmannian $\mathrm{G}_{m}\left(T_{q} N\right)=\hat{N}_{q}$ with respect to the reference point $W$. It remains to prove the analogous Formula (95) for the Grassmannian $\mathrm{G}_{m}\left(T_{q}\right)=\hat{N}_{q}$. This is done easily: If $\tilde{g} \in \mathrm{O}\left(T_{q} N\right)$ is given and we put $g:=$ $u^{-1} \circ \tilde{g} \circ u \in \mathrm{O}\left(\mathbb{R}^{n}\right)$, then we have $\psi_{\tilde{g}}=\hat{\rho}_{u} \circ \psi_{g} \circ\left(\hat{\rho}_{u}\right)^{-1}$, which is an isometry of $\hat{N}_{q}$.

For (b). We choose a parallel orthonormal frame field $\tilde{c}: J \rightarrow \mathrm{L}(N)$ of the riemannian manifold $N$ along $c$. The horizontal displacement under consideration is then given by

$$
\underset{0}{(\| c)^{\hat{\mathcal{H}}}}=\hat{\rho}_{\tilde{c}\left(t_{2}\right)} \circ\left(\hat{\rho}_{\tilde{c}\left(t_{1}\right)}\right)^{-1}
$$

see (85) and [KN] Vol. I, p. 88. Thus (b) follows from (a)(iii).

For (c) and (d). These assertions are valid for every riemannian submersion $\tau$; for (c) see [ON1]. - Equation (100) is the relation between the second fundamental form and the shape operator (well known from submanifold geometry); it is verified by means of the Ricci equation and Formula (98). In order to prove (101) we rearrange it to

$$
\tau_{*} \nabla_{\hat{U}}^{L C} \hat{X}-\nabla_{\hat{U}}^{N} \tau_{*} \hat{X}=-\tau_{*} S_{\hat{U}}^{\hat{H}} \hat{X}
$$

As both sides of this formula are linear in the variable $\hat{X}$, we may suppose that $\hat{X}$ is the horizontal lift of a vector field $X \in \Gamma(T N)$; then we have $\nabla^{N} \hat{U}_{*} \hat{X}=\nabla_{\tau_{*}}^{N} X=0$ and $[\hat{U}, \hat{X}] \in \Gamma(\hat{\mathcal{V}})$. Thus we get

$$
\tau_{*} \nabla_{\hat{U}}^{L C} \hat{X}-\nabla_{\hat{U}}^{N} \tau_{*} \hat{X}=\tau_{*}\left(\nabla_{\hat{X}}^{L C} \hat{U}+[\hat{U}, \hat{X}]\right)=-\tau_{*} S_{\hat{U}}^{\hat{\mathcal{A}}} \hat{X} .
$$

For (e). First we prove Equation (103): Both sides of (103) are linear in $\hat{X}$. Thus it suffices to verify it for the horizontal lift $\hat{X}$ of some vector field $X \in \Gamma(T N)$. Let $\left(\hat{\Phi}_{t}\right)$ and $\left(\Phi_{t}\right)$ denote the local 1-parameter groups of $\hat{X}$ and $X$, respectively; then we have $\tau \circ \hat{\Phi}_{t}=\Phi_{t} \circ \tau$, and this fact implies that for every $W \in \hat{N} \quad \hat{\Phi}_{t *} \mid \hat{\mathcal{V}}_{W}$ is an isomorphism $\hat{\mathcal{V}}_{W} \rightarrow \hat{\mathcal{V}}_{\hat{\Phi}_{t}(W)}$. Let $\xi_{W}: J \rightarrow \hat{N}$ be the maximal integral curve of $\hat{X}$ with $\xi_{W}(0)=W$ and $c:=\tau \circ \xi_{W}$. Then $t \mapsto \hat{\Phi}_{t}(W)=\xi_{W}(t)$ is the horizontal lift of $c$ with initial point $W$; thus $\hat{\Phi}_{t} \mid \hat{N}_{c(0)}: \hat{N}_{c(0)} \rightarrow \hat{N}_{c(t)}$ is the $\hat{\mathcal{H}}$-horizontal displacement. Because of (b), therefore the isomorphism $\hat{\Phi}_{t *} \mid \hat{\mathcal{V}}_{W}: \hat{\mathcal{V}}_{W} \rightarrow \hat{\mathcal{V}}_{\hat{\Phi}_{t}(W)}$ is isometric, 
and we get

$$
\begin{aligned}
\hat{X}_{W} \cdot\left\langle\hat{U}_{1}, \hat{U}_{2}\right\rangle & =\left(\left\langle\hat{U}_{1}, \hat{U}_{2}\right\rangle \circ \xi_{W}\right)^{\prime}(0)=\left.\frac{\mathrm{d}}{\mathrm{d} t}\right|_{t=0}\left\langle\hat{U}_{1} \circ \hat{\Phi}_{t}(W), \hat{U}_{2} \circ \hat{\Phi}_{t}(W)\right\rangle \\
& =\left.\frac{\mathrm{d}}{\mathrm{d} t}\right|_{t=0}\left\langle\hat{\Phi}_{t *}^{-1} \hat{U}_{1} \circ \hat{\Phi}_{t}(W), \hat{\Phi}_{t *}^{-1} \hat{U}_{2} \circ \hat{\Phi}_{t}(W)\right\rangle \\
& =\left\langle\left.\frac{\mathrm{d}}{\mathrm{d} t}\right|_{t=0} \hat{\Phi}_{t *}^{-1} \hat{U}_{1} \circ \hat{\Phi}_{t}(W), \hat{U}_{2}(W)\right\rangle+\left\langle\hat{U}_{1}(W),\left.\frac{\mathrm{d}}{\mathrm{d} t}\right|_{t=0} \hat{\Phi}_{t *}^{-1} \hat{U}_{2} \circ \hat{\Phi}_{t}(W)\right\rangle
\end{aligned}
$$

Now, the term $\left.\frac{\mathrm{d}}{\mathrm{d} t}\right|_{t=0} \hat{\Phi}_{t *}^{-1} \hat{U}_{k} \circ \hat{\Phi}_{t}(W)$ defines the Lie derivative $L_{\hat{X}} \hat{U}_{k}$ at the point $W$, which coincides with the Lie bracket $\left[\hat{X}, \hat{U}_{k}\right]_{W}$ (see $[\mathrm{KN}]$ Vol. I, p. 29). Substituting the Lie bracket in the preceding formula we get Equation (103). - In order to prove that $\hat{\mathcal{V}}$ is a $\nabla^{L C}$-autoparallel subbundle of $T \hat{N}$ let $\hat{X}, \hat{U}_{1}, \hat{U}_{2}$ be as before, in particular $\hat{X}$ is some horizontal lift. Then $\left[\hat{U}_{1}, \hat{U}_{2}\right],\left[\hat{U}_{1}, \hat{X}\right]$ and $\left[\hat{U}_{2}, \hat{X}\right]$ are vertical. Thus by the formula of Koszul for the Levi Civita connection (see [ON2], p. 61) we get:

$$
\begin{aligned}
2 \cdot\left\langle\nabla_{\hat{U}_{1}}^{L C} \hat{U}_{2}, \hat{X}\right\rangle= & \left(\hat{U}_{1}\left\langle\hat{U}_{2}, \hat{X}\right\rangle-\left\langle\hat{U}_{1},\left[\hat{U}_{2}, \hat{X}\right]\right\rangle\right) \\
& +\left(\hat{U}_{2}\left\langle\hat{U}_{1}, \hat{X}\right\rangle-\left\langle\hat{U}_{2},\left[\hat{U}_{1}, \hat{X}\right]\right\rangle\right)-\left(\hat{X}\left\langle\hat{U}_{1}, \hat{U}_{2}\right\rangle-\left\langle\hat{X},\left[\hat{U}_{1}, \hat{U}_{2}\right]\right\rangle\right) \\
= & -\left(\left\langle\hat{U}_{1},\left[\hat{U}_{2}, \hat{X}\right]\right\rangle+\left\langle\hat{U}_{2},\left[\hat{U}_{1}, \hat{X}\right]\right\rangle+\hat{X}\left\langle\hat{U}_{1}, \hat{U}_{2}\right\rangle\right) \\
= & -\left(\hat{X}\left\langle\hat{U}_{1}, \hat{U}_{2}\right\rangle-\left\langle\hat{U}_{1},\left[\hat{X}, \hat{U}_{2}\right]\right\rangle-\left\langle\hat{U}_{2},\left[\hat{X}, \hat{U}_{1}\right]\right\rangle\right) \underset{(103)}{=} 0,
\end{aligned}
$$

hence $\nabla_{\hat{U}_{1}}^{L C} \hat{U}_{2} \in \Gamma(\hat{\mathcal{V}})$; that means the autoparallelity of the subbundle $\hat{\mathcal{V}}$. As the fibres $\hat{N}_{q}$ are integral manifolds of $\hat{\mathcal{V}}$, we see that they are totally geodesic submanifolds of $\hat{N}$. Furthermore, since the torsion of $\nabla^{L C}$ vanishes and $\hat{\mathcal{V}}$ is $\hat{\nabla}$-autoparallel we get

$$
\left\langle\left[\hat{X}, \hat{U}_{1}\right], \hat{U}_{2}\right\rangle=\left\langle\nabla_{\hat{X}}^{L C} \hat{U}_{1}-\nabla_{\hat{U}_{1}}^{L C} \hat{X}, \hat{U}_{2}\right\rangle=\left\langle\nabla_{\hat{X}}^{L C} \hat{U}_{1}, \hat{U}_{2}\right\rangle+\left\langle\hat{X}, \nabla_{\hat{U}_{1}}^{L C} \hat{U}_{2}\right\rangle=\left\langle\nabla_{\hat{X}}^{L C} \hat{U}_{1}, \hat{U}_{2}\right\rangle .
$$

This result implies Equation (102). Finally Equation (103) can also be deduced from Equation (102) by applying the Ricci identity.

For (f). This statement is true for every riemannian submersion $\tau$ with totally geodesic fibres. For the proof we choose a further vector field $\hat{U}^{\prime} \in \Gamma(\hat{\mathcal{V}})$. Since $\hat{\mathcal{V}}$ is autoparallel, we obtain by means of the Ricci identity

$$
\left\langle\nabla_{\hat{U}}^{L C} \hat{X}, \hat{U}^{\prime}\right\rangle=-\left\langle\hat{X}, \nabla_{\hat{U}}^{L C} \hat{U}^{\prime}\right\rangle=0
$$

which implies (104).

In the following we will also use the inner product

$$
\left\langle\left\langle A_{1}, A_{2}\right\rangle\right\rangle=-\operatorname{trace}\left(A_{2} \circ A_{1}\right)
$$

for all endomorphisms $A_{1}, A_{2} \in \operatorname{End}_{-}\left(T_{q} N\right)$. It is well known that for every $W \in \hat{N}_{q}$ one has

$$
\mathbb{H}_{W} \perp \mathbb{M}_{W} ;
$$

this can easily been proved by use of an orthonormal frame $u \in \mathbb{P}_{W}$.

In [ON1] O'NeILL has derived relations between the curvature tensors of the bundle space and base space of a riemannian submersion. For our investigation the following will become important. 
Lemma 7 (A relation between the curvature of $\hat{\mathcal{H}}$ and $\boldsymbol{N}$ ). For all $W \in \hat{N}, \hat{x}, \hat{y} \in \hat{\mathcal{H}}_{W}$ and $\hat{u} \in \hat{\mathcal{V}}_{W}$ we have

$$
-2 \cdot\left\langle S_{\hat{u}}^{\hat{\hat{u}}} \hat{x}, \hat{y}\right\rangle=\langle\hat{\Omega}(\hat{x}, \hat{y}), \hat{u}\rangle=\left\langle\left\langle R^{N}(x, y), \hat{\nu}(\hat{u})\right\rangle\right\rangle
$$

with $x:=\tau_{*} \hat{x}$ and $y:=\tau_{*} \hat{y}$.

Proof. the first part of (109) is a repetition of (100). Furthermore, using Equation (78), (77) and Remark 11 we calculate:

$$
\forall \hat{x}, \hat{y} \in \hat{\mathcal{H}}_{W}: \mathbb{M}_{W} \text {-part of } R^{N}(x, y)=\mathrm{d} \hat{\nu}(\hat{x}, \hat{y})=\hat{\nu}(\hat{\Omega}(\hat{x}, \hat{y})) .
$$

Therefore, Theorem 4 gives $\hat{\Omega}(\hat{x}, \hat{y})=\Theta_{W}\left(\mathbb{M}_{W}\right.$-part of $R^{N}(x, y)$. Moreover it implies $\hat{u}=$ $\Theta(\hat{\nu}(\hat{u}))$; therefore we get:

$$
\begin{aligned}
\langle\hat{\Omega}(\hat{x}, \hat{y}), \hat{u}\rangle & =\left\langle\Theta\left(\mathbb{M}_{W} \text {-part of } R^{N}(x, y)\right), \Theta(\hat{\nu}(\hat{u}))\right\rangle \\
& =\left\langle\left\langle\mathbb{M}_{W} \text {-part of } R^{N}(x, y), \hat{\nu}(\hat{u})\right\rangle\right\rangle .
\end{aligned}
$$

If we split $R^{N}(x, y)=\left(\mathbb{M}_{W}\right.$-part of $\left.R^{N}(x, y)\right)+\left(\mathbb{H}_{W}\right.$-part of $\left.R^{N}(x, y)\right)$ and take (108) into account, we obtain the second part of (109).

Now we will compare the two connections $\hat{\nabla}$ and $\nabla^{L C}$. At first we see that these connections are different, because according to Proposition 10 the torsion of $\hat{\nabla}$ does not vanish identically. For our comparison we introduce the difference tensor

$$
\Delta:=\nabla^{L C}-\hat{\nabla}
$$

Theorem 5. (a) $\hat{\nabla}$ is a metric connection on the riemannian manifold $\hat{N}$. Therefore we have in particular

$$
\forall W \in \hat{N}, \hat{u}, \hat{v}, \hat{w} \in T_{W} \hat{N}:\langle\Delta(\hat{u}, \hat{v}), \hat{w}\rangle+\langle\Delta(\hat{u}, \hat{w}), \hat{v}\rangle=0 .
$$

(b) The following holds: $\forall \hat{U}_{1}, \hat{U}_{2} \in \Gamma(\hat{\mathcal{V}}): \quad \hat{\nabla}_{\hat{U}_{1}} \hat{U}_{2}=\nabla_{\hat{U}_{1}}^{L C} \hat{U}_{2} \in \Gamma(\hat{\mathcal{V}})$.

Therefore, for every $q \in N$ the fibre $\hat{N}_{q}=\mathrm{G}_{m}\left(T_{q} N\right)$ is an affine (= autoparallel) submanifold of $(\hat{N}, \hat{\nabla})$,

$$
\Delta \mid\left(\hat{\mathcal{V}} \times_{\hat{N}} \hat{\mathcal{V}}\right)=0
$$

and on $\hat{N}_{q}$ the connection $\hat{\nabla}$ coincides with the connection which $\hat{N}_{q}$ bears canonically as symmetric space (see [KN] Vol.2, p. 230).

For the following Equations (116) and (117) we fix a subspace $W \in \hat{N}_{q}(q \in N)$, choose $\hat{x}, \hat{y} \in \hat{\mathcal{H}}_{W}$ and $\hat{u} \in \hat{\mathcal{V}}_{W}$ and put $x:=\tau_{*} \hat{x}$ and $y:=\tau_{*} \hat{y} \in T_{q} N$.

(c) For all vector fields $\hat{X}, \hat{Y} \in \Gamma(\hat{\mathcal{H}})$ and $\hat{U} \in \Gamma(\hat{\mathcal{V}})$ the following formulas hold:

$$
\begin{aligned}
\nabla_{\hat{X}}^{L C} \hat{Y} & =\hat{\nabla}_{\hat{X}} \hat{Y}-\frac{1}{2} \cdot \hat{\Omega}(\hat{X}, \hat{Y}) \quad \text { and } \\
\nabla_{\hat{X}}^{L C} \hat{U} & =-S_{\hat{U}}^{\hat{H}} \hat{X}+\hat{\nabla}_{\hat{X}} \hat{U} .
\end{aligned}
$$

Because of $\hat{\nabla}_{\hat{X}} \hat{Y}, S_{\hat{U}}^{\hat{\mathcal{H}}} \hat{X} \in \Gamma(\hat{\mathcal{H}})$ and $\hat{\nabla}_{\hat{X}} \hat{U}, \hat{\Omega}(\hat{X}, \hat{Y}) \in \Gamma(\hat{\mathcal{V}})$ the Formulas (114) and (115) can be interpreted as Gauß and Weingarten equation of the subbundle $\hat{\mathcal{H}}$ in the 
riemannian manifold $\hat{N}$; then $\hat{\nabla} \mid(\hat{\mathcal{H}} \times \Gamma(\hat{\mathcal{H}}))$ and $\hat{\nabla} \mid(\hat{\mathcal{H}} \times \Gamma(\hat{\mathcal{V}}))$ play the role of a "semi-connection" on $\hat{\mathcal{H}}$ resp. on the normal bundle $\hat{\mathcal{V}}$ of $\hat{\mathcal{H}}$. The quantities $S^{\hat{\mathcal{H}}}$ and $-\frac{1}{2} \cdot \hat{\Omega} \mid(\hat{\mathcal{H}} \times \Gamma(\hat{\mathcal{H}}))$ were already interpreted as shape operator and second fundamental form of the subbundle $\hat{\mathcal{H}}$ in Proposition 12. The Formulas (114) and (115) can also be expressed by

$$
\Delta(\hat{x}, \hat{y})=-\frac{1}{2} \cdot \hat{\Omega}(\hat{x}, \hat{y}) \in \hat{\mathcal{V}}_{W} \quad \text { and } \quad \Delta(\hat{x}, \hat{u})=-S_{\hat{u}}^{\hat{\mathcal{H}}} \hat{x} \in \hat{\mathcal{H}}_{W}
$$

for all choices of $\hat{x}, \hat{y} \in \hat{\mathcal{H}}_{W}$ and $\hat{u} \in \hat{\mathcal{V}}_{W}$.

(d) $\Delta(\hat{u}, \hat{x}) \in \hat{\mathcal{H}}_{W} \quad$ and $\quad\langle\Delta(\hat{u}, \hat{x}), \hat{y}\rangle=\frac{1}{2} \cdot\left\langle\left\langle R^{N}(x, y)-x \wedge y, \hat{\nu}(\hat{u})\right\rangle\right\rangle$

Proof. For (a). The background for this statement is that the tangent bundle $T \hat{N}$ is associated with the principal bundle $\mathbb{P}$ via the map $\rho^{T \hat{N}}$ and that $\hat{\nabla}$ and the riemannian metric of $\hat{N}$ are defined by means of this association. In order to prove it explicitly we use that this statement is equivalent to the following claim: For every curve $\xi: J \rightarrow \hat{N}$ and every $\hat{\nabla}$-parallel vector fields $\hat{Y}_{1}, \hat{Y}_{2} \in \Gamma_{\xi}(T \hat{N})$ the function $\left\langle\hat{Y}_{1}, \hat{Y}_{2}\right\rangle$ is constant. For verifying the last statement, we use the description (91) for $\hat{\nabla}$-parallel vector fields choosing a horizontal lift $u_{\xi}: J \rightarrow \mathbb{P}$ of $\xi$ and suitable elements $\left(x_{i}, A_{i}\right) \in \mathbb{R}^{n} \oplus \mathfrak{m}$ such that we have $\hat{Y}_{i}(t)=\rho_{u_{\xi}(t)}^{T \hat{N}}\left(x_{i}, A_{i}\right)$. Formula (97) then implies $\left\langle\hat{Y}_{1}, \hat{Y}_{2}\right\rangle \equiv\left\langle\left\langle\left(x_{1}, A_{1}\right),\left(x_{2}, A_{2}\right)\right\rangle\right\rangle$. - Formula (111) follows now, because $\hat{\nabla}$ and $\nabla^{L C}$ both are metric connections.

For (b). As mentioned above $\hat{\mathcal{V}}$ is a $\hat{\nabla}$-parallel subbundle of $T \hat{N}$. In particular, it is autoparallel, i.e., we have

$$
\forall \hat{U}_{1}, \hat{U}_{2} \in \Gamma(\hat{\mathcal{V}}): \quad \hat{\nabla}_{\hat{U}_{1}} \hat{U}_{2} \in \Gamma(\hat{\mathcal{V}}) ;
$$

therefore, its integral manifolds, i.e. the fibres $\hat{N}_{q}$, are affine submanifolds of $\hat{N}$. Furthermore, for all vectors $\hat{u}_{1}, \hat{u}_{2} \in T_{W} \hat{N}_{q}$ we have $\tau_{*} \hat{u}_{i}=0$, and therefore the torsion of $\hat{N}_{q}$ (equipped with the connection $\nabla^{\hat{N}_{q}}$ induced by $\left.\hat{\nabla}\right)$ vanishes by means of $(93)$, since $T^{\hat{N}_{q}}\left(\hat{u}_{1}, \hat{u}_{2}\right)=T^{\hat{N}}\left(\hat{u}_{1}, \hat{u}_{2}\right)$. Because of (a) $\nabla^{\hat{N}_{q}}$, therefore, is the Levi-Civita connection of the totally geodesic submanifold $\hat{N}_{q}$ of $\hat{N}$. This fact implies

$$
\forall \hat{U}_{1}, \hat{U}_{2} \in \Gamma(\hat{\mathcal{V}}): \quad \hat{\nabla}_{\hat{U}_{1}} \hat{U}_{2}=\nabla_{\hat{U}_{1}}^{L U_{2}}
$$

thus (112) is proved. The further statement of (b) follows from Proposition 12(a)(iii), because the Levi-Civita connection of the canonical riemannian metric of $\mathrm{G}_{m}\left(T_{q} N\right)$ is the canonical connection of this symmetric space.

For (c). The Equations (114) and (115) obviously will follow from the four equations

$$
\begin{aligned}
& \text { (i) } \eta\left(\nabla^{L C} \hat{X} \hat{Y}\right)=\hat{\nabla}_{\hat{X}} \hat{Y} \\
& \text { (iii) } \eta\left(\nabla_{\hat{X}}^{L C} \hat{U}\right)=-S_{\hat{U}}^{\hat{\mathcal{A}}} \hat{X} \\
& \text { (ii) } \nu\left(\nabla^{L C} \hat{X} \hat{Y}\right)=-\frac{1}{2} \cdot \hat{\Omega}(\hat{X}, \hat{Y}) \\
& \text { (iv) } \nu\left(\nabla_{\hat{X}}^{L C} \hat{U}\right)=\hat{\nabla}_{\hat{X}} \hat{U}
\end{aligned}
$$

The Equations (ii) and (iii) are true as was shown in Proposition 12(c) and (d). Furthermore, from the first part of (92), Remark 11(i) and O'Neills Formula (99) we get $\tau_{*} \hat{\nabla}_{\hat{X}} \hat{Y}=\nabla^{N} \hat{X}_{*} \hat{Y}-$ $\hat{\nu}(\hat{X})\left(\tau_{*} \hat{Y}\right)=\nabla^{N}{ }_{\hat{X}} \tau_{*} \hat{Y}=\tau_{*} \nabla^{L C} \hat{X} \hat{Y}$; because of $\hat{\nabla}_{\hat{X}} \hat{Y} \in \Gamma(\hat{\mathcal{H}})$ therefore Equation (i) is true. Finally, we calculate by means of Formula (102)

$$
\nu\left(\nabla_{\hat{X}}^{L C} \hat{U}\right)=\nu[\hat{X}, \hat{U}]=\nu\left(\hat{\nabla}_{\hat{X}} \hat{U}-\hat{\nabla}_{\hat{U}} \hat{X}-T^{\hat{N}}(\hat{X}, \hat{U})\right) .
$$


Because of Equation (93) we have $\hat{\nu}\left(T^{\hat{N}}(\hat{X}, \hat{U})\right)=0$, and therefore Theorem 4 implies $\nu\left(T^{\hat{N}}(\hat{Y}, \hat{U})\right)=0$; moreover we have $\hat{\nabla}_{\hat{U}} \hat{X} \in \Gamma(\hat{\mathcal{H}})$ and $\hat{\nabla}_{\hat{X}} \hat{U} \in \Gamma(\hat{\mathcal{V}})$; thus Equation (iv) is true.

For (d). The first part of (117) follows from Proposition 12(f) and the $\hat{\nabla}$-parallelity of $\hat{\mathcal{H}}$. Furthermore, if we combine the first part of Formula (92) with (101) we find $\tau_{*} \Delta(\hat{u}, \hat{x})=$ $\hat{\nu}(\hat{u}) x-\tau_{*} S_{\hat{u}}^{\hat{\mathcal{H}}} \hat{x}$ and therefore

$$
\langle\Delta(\hat{u}, \hat{x}), \hat{y}\rangle=\langle\hat{\nu}(\hat{u}) x, y\rangle-\left\langle S_{\hat{u}}^{\hat{H}} \hat{x}, \hat{y}\right\rangle .
$$

The last term of (118) is known from (109). Thus the second part of (117) follows with the following fact: If $V$ is a euclidean vector space, then we have for all vectors $x, y \in V$ and every endomorphism $A \in$ End_- $\left._{-}\right)$

$$
\langle A x, y\rangle=-\frac{1}{2} \cdot\langle\langle x \wedge y, A\rangle\rangle .
$$

The proof of this equation is an easy task, if one chooses an orthonormal basis $\left(a_{1}, \ldots, a_{n}\right)$ of $V$ and writes $x=\sum\left\langle x, a_{i}\right\rangle \cdot a_{i}$ and $y=\sum\left\langle y, a_{j}\right\rangle \cdot a_{j}$.

Corollary $\mathbf{8}$ (Comparison of $\hat{\nabla}$ with $\nabla^{L C}$ ). The difference tensor $\Delta$ is determined by the following formula valid for all triples $(\hat{u}, \hat{v}, \hat{w}) \in T \hat{N} \times{ }_{\hat{N}} T \hat{N} \times{ }_{\hat{N}} T \hat{N}$ :

$$
\langle\Delta(\hat{u}, \hat{v}), \hat{w}\rangle=\frac{1}{2} \cdot\left(-\left\langle\left\langle R^{N}(u, v), \hat{\nu}(\hat{w})\right\rangle\right\rangle+\left\langle\left\langle R^{N}(u, w), \hat{\nu}(\hat{v})\right\rangle\right\rangle+\left\langle\left\langle R^{N}(v, w)-v \wedge w, \hat{\nu}(\hat{u})\right\rangle\right\rangle\right),
$$

where we have defined $u:=\tau_{*} \hat{u}, v:=\tau_{*} \hat{v}$ and $w:=\tau_{*} \hat{w}$.

Proof. The equation can be proved by checking the eight cases, in which the vectors $\hat{u}, \hat{v}$ and $\hat{w}$ are vertical or horizontal. Obviously the right-hand side of the equation vanishes, if more than one vector is vertical or if all vectors are horizontal; in these cases also the left-hand side vanishes because of (113), (116) and (117). The case $(\hat{u}, \hat{v}, \hat{w}) \in \hat{\mathcal{V}}_{W} \times \hat{\mathcal{H}}_{W} \times \hat{\mathcal{H}}_{W}$ follows from (117). So we are left with two cases to be treated: For $(\hat{u}, \hat{v}, \hat{w}) \in \hat{\mathcal{H}}_{W} \times \hat{\mathcal{H}}_{W} \times \hat{\mathcal{V}}_{W}$ we obtain from (116) and Lemma 7: $\langle\Delta(\hat{u}, \hat{v}), \hat{w}\rangle=-\frac{1}{2} \cdot\langle\hat{\Omega}(\hat{u}, \hat{v}), \hat{w}\rangle=-\frac{1}{2} \cdot\left\langle\left\langle R^{N}(u, v), \hat{\nu}(\hat{w})\right\rangle\right\rangle=$ right-hand side of the equation; and the case $(\hat{u}, \hat{v}, \hat{w}) \in \hat{\mathcal{H}}_{W} \times \hat{\mathcal{V}}_{W} \times \hat{\mathcal{H}}_{W}$ follows from the previous one because both sides of our equation are skew-symmetric with respect to the variables $\hat{v}$ and $\hat{w}(\operatorname{see}(111))$.

But nevertheless perhaps there is yet a close geometric relation between the connections $\nabla^{L C}$ and $\hat{\nabla}$. To explain the idea let us call in mind how the connection $\hat{\nabla}$ was defined. We started from the Levi-Civita connection on $N$, considered its "pull back connection" on the pull back bundle $\mathbb{E}=\tau^{*} T N$ and defined in usual way (i.e., by projection) the connections $\nabla^{\top}, \nabla^{\perp}$ on the subbundles $\mathbb{T}, \Perp \subset \mathbb{E}$ (see (63)); from these connections we obtained the connection $\nabla^{\mathbb{M}}$ on the bundle $\mathbb{M} \cong \mathrm{L}\left(\mathbb{T}, \mathbb{\Perp}\right.$ ) (see (70) and (70)). Thus we got the connection $\nabla^{\top} \oplus \nabla^{\perp} \oplus \nabla^{\mathbb{M}}$ on the bundle $\mathbb{E} \oplus \mathbb{M}=\pi \oplus \Perp \oplus \mathbb{M}$. Finally the connection $\hat{\nabla}$ was constructed by pulling back the previous connection via the isomorphism $(\hat{\eta}, \hat{\nu}): T \hat{N} \rightarrow \mathbb{E} \oplus \mathbb{M}$ (see Theorem 4). On the other hand, via this isomorphism the splitting $\pi \oplus \Perp \oplus \mathbb{M}$ corresponds to the splitting $T \hat{N}=\mathcal{D} \oplus \mathcal{D}^{\perp} \oplus \hat{\mathcal{V}}$; see Remark 12. This remark stated that the three subbundles $\mathcal{D}, \mathcal{D}^{\perp}$ and $\hat{\mathcal{V}}$ are $\hat{\nabla}$-parallel; we also see now that the notation $\mathcal{D}^{\perp}$ was justified because the construction of the riemannian metric via the map $\rho^{T \hat{N}}$ implies that

$$
\text { the splitting } T \hat{N}=\mathcal{D} \oplus \mathcal{D}^{\perp} \oplus \hat{\mathcal{V}} \text { is an orthogonal splitting. }
$$


After this description it is natural to wonder whether the connection $\hat{\nabla}$ conicides with the connection $\nabla^{\mathcal{D}} \oplus \nabla^{\mathcal{D}^{\perp}} \oplus \nabla^{\hat{\mathcal{V}}}$, where the connections $\nabla^{\mathcal{D}}, \nabla^{\mathcal{D}^{\perp}}, \nabla^{\hat{\mathcal{V}}}$ are induced by $\nabla^{L C}$ (by projection). Because the subbundles $\mathcal{D}, \mathcal{D}^{\perp}$ and $\hat{\mathcal{V}}$ are $\hat{\nabla}$-parallel, this question is equivalent to the set of following three "partial questions":

Question 1. $\forall(\hat{w}, \hat{u}, \hat{v}) \in T \hat{N} \times_{\hat{N}} \hat{\mathcal{V}} \times_{\hat{N}} \hat{\mathcal{V}}:\langle\Delta(\hat{w}, \hat{u}), \hat{v}\rangle=0 \quad ?$

Question 2. $\forall(\hat{w}, \hat{x}, \hat{y}) \in T \hat{N} \times_{\hat{N}} \hat{\mathcal{D}} \times_{\hat{N}} \hat{\mathcal{D}}:\langle\Delta(\hat{w}, \hat{x}), \hat{y}\rangle=0 \quad ?$

Question 3. $\forall(\hat{w}, \hat{x}, \hat{y}) \in T \hat{N} \times{ }_{\hat{N}} \hat{\mathcal{D}}^{\perp} \times_{\hat{N}} \hat{\mathcal{D}}^{\perp}:\langle\Delta(\hat{w}, \hat{x}), \hat{y}\rangle=0 \quad ?$

Corollary 9 (Answers to the questions). Question 1 has always a positive answer. Question 2 resp. Question 3 is true at a "point" $W \in \hat{N}$ if and only if $W$ resp. $W^{\perp}$ is a curvature invariant subspace of $T_{\tau(W)} N$. Therefore in case $m \geq 2$ the connections $\hat{\nabla}$ and $\nabla^{\mathcal{D}} \oplus \nabla^{\mathcal{D}^{\perp}} \oplus \nabla^{\hat{v}}$ coincide on the whole manifold $\hat{N}$ if and only $N$ is a space of constant curvature.

Proof. The answer of Question 1 follows immediately from Corollary 8. The answers to the other two questions are based on the the assertion

$$
\forall \hat{x}, \hat{y} \in \hat{\mathcal{H}}_{W}:\left(\left(\forall \hat{u} \in \hat{\mathcal{V}}_{W}:\langle\Delta(\hat{u}, \hat{x}), \hat{y}\rangle=0\right) \Longleftrightarrow R^{N}(x, y)-x \wedge y \in \mathbb{H}_{W}\right),
$$

which follows from the second part of (117), because $\mathbb{H}_{W} \oplus \mathbb{M}_{W}$ is an orthogonal splitting of End ${ }_{-}\left(T_{\tau(W)} N\right)$ (because of $\left.(108)\right)$ and $\mathbb{M}_{W}=\hat{\nu}\left(\hat{\mathcal{V}}_{W}\right)$. By this result we get:

$$
\begin{aligned}
& \forall(\hat{w}, \hat{x}, \hat{y}) \in T_{W} \hat{N} \times \mathcal{D}_{W} \times \mathcal{D}_{W}:\langle\Delta(\hat{w}, \hat{x}), \hat{y}\rangle=0 \\
& \Longleftrightarrow \forall(\hat{u}, \hat{x}, \hat{y}) \in \hat{\mathcal{V}}_{W} \times \mathcal{D}_{W} \times \mathcal{D}_{W}:\langle\Delta(\hat{u}, \hat{x}), \hat{y}\rangle=0 \\
& \Longleftrightarrow \forall(\hat{x}, \hat{y}) \in \mathcal{D}_{W} \times \mathcal{D}_{W}: R^{N}(x, y)-x \wedge y \in \mathbb{H}_{W} \\
& \Longleftrightarrow \forall(\hat{x}, \hat{y}) \in \mathcal{D}_{W} \times \mathcal{D}_{W}: R^{N}(x, y) \in \mathbb{H}_{W} \\
& \Longleftrightarrow W \text { is curvature invariant }
\end{aligned}
$$

for the first equivalence we have used the first part of (117), and for the third equivalence notice that we have $x \wedge y \in \mathbb{H}_{W}$ if both vectors $x$ and $y$ lie in $W$ resp. in $W^{\perp}$. (The characterization of the curvature invariance of $W$ in the last equivalence has a further consequence, see footnote $^{12}$.) Hereby our answer of Question 2 is proved. For Question 3 we can use the same argumentation only replacing $\mathcal{D}$ by $\mathcal{D}^{\perp}$.

The last statement of the corollary is true, because $n$-dimensional riemannian spaces $N$ of constant curvature are characterized by the fact that all subspaces $W \in \mathrm{G}_{m}(T N)$ for some dimension $m \in\{2, \ldots, n-1\}$ are curvature invariant (already proved by É. CARTAN in $\mathrm{N}^{\circ} .108$ of his book $[\mathrm{C}])$.

Theorem 6 (The second fundamental form of a Gauß map). If $M$ is an $m$-dimensional submanifold of $N$, then the second fundamental form $h^{\xi}$ of its Gauß map $\xi:=\xi^{M}: M \rightarrow \hat{N}$ characterized by the "Gauß equation"

$$
\hat{\nabla}_{X} \xi_{*} Y=\xi_{*} \nabla^{M} Y+h^{\xi}(X, Y) \text { for all } X, Y \in \Gamma(T M)
$$

\footnotetext{
${ }^{12}$ From the Equations (108) and (109) and the definition of $\mathcal{D}$ it follows that $W$ is curvature invariant if and only if $\forall \hat{v}_{1}, \hat{v}_{2} \in \mathcal{D}_{W}: \hat{\Omega}\left(\hat{v}_{1}, \hat{v}_{2}\right)=0$ holds. This result can also be found in the proof of Theorem 5 in [PR1].
} 
is related to the covariant derivative $\bar{\nabla} h^{M}$ of the second fundamental form of $M$ by

$$
h_{p}^{\xi}(u, v)=\Theta_{\xi(p)}\left(A_{v}^{b}\right) \quad \text { with } \quad b:=\bar{\nabla}_{u} h^{M} \quad \text { for all } \quad u, v \in T_{p} M(p \in M) .
$$

In particular, $h^{\xi}$ takes its values in the vertical subbundle $\hat{\mathcal{V}}$.

Proof. Let vector fields $X, Y, Z \in \Gamma(T M)$ be fixed. We use (92), (79), (70) and $\tau \circ \xi=M \hookrightarrow N$. First we obtain $\tau_{*} h^{\xi}(X, Y)=\nabla^{N} Y-\hat{\nu}\left(\xi_{*} X\right) Y-\nabla^{M}{ }_{X} Y=h^{M}(X, Y)-\hat{\nu}\left(\xi_{*} X\right) Y=0$; thus $h^{\xi}$ is vertical. Furthermore,

$$
\begin{aligned}
\hat{\nu}\left(h^{\xi}(X, Y)\right) Z & =\left(\nabla^{\mathbb{M}}{ }_{X} \hat{\nu}\left(\xi_{*} Y\right)\right) Z-\hat{\nu}\left(\xi_{*} \nabla^{M}{ }_{X} Y\right) Z \\
& =\nabla^{\mathbb{E}}{ }_{X}\left(\hat{\nu}\left(\xi_{*} Y\right) Z\right)-\hat{\nu}\left(\xi_{*} Y\right)\left(\nabla^{\mathbb{E}} X\right)-\hat{\nu}\left(\xi_{*} \nabla^{M}{ }_{X} Y\right) Z \\
& =\nabla^{\perp M}{ }_{X}\left(h^{M}(Y, Z)\right)-h^{M}\left(Y, \nabla^{M}{ }_{X} Z\right)-h^{M}\left(\nabla^{M}{ }_{X} Y, Z\right)=\left(\bar{\nabla}_{X} h^{M}\right)(Y, Z) ;
\end{aligned}
$$

thus $\hat{\nu}\left(h^{\xi}(u, v)\right)=A_{v}^{b}$ with $b:=\bar{\nabla}_{u} h^{M}$ for all $u, v \in T_{p} M$. Since $h^{\xi}$ is vertical, Theorem 4 shows $\Theta\left(A_{v}^{b}\right)=\nu\left(h^{\xi}(u, v)\right)=h^{\xi}(u, v)$.

Corollary 10 (Characterization of II-parallelity by the Gauß map). An isometric immersion $f: M \rightarrow N$ with $\operatorname{dim} M=m$ is II-parallel if and only if its Gauß map $\xi:=\xi^{f}: M \rightarrow \hat{N}, p \mapsto$ $f_{*} T_{p} M$ is affine, i.e., if the second fundamental form $h^{\xi}$ vanishes (see [KN] Vol.1, p. 225).

Proof. As we may argue locally, we may restrict the consideration to the case that $f$ is the inclusion of a submanifold $M \subset N$. Because $\Theta_{\xi(p)}: \mathbb{M}_{\xi(p)} \rightarrow \hat{\mathcal{V}}_{\xi(p)}$ is an isomorphism, Theorem 6 shows that $\xi^{M}$ is affine if and only if $\bar{\nabla} h^{M}=0$.

Remark 13. (a) Theorem 6 and Corollary 10 are generalizations of results, which J. VILMS discovered for submanifolds of the euclidean space $\mathbb{R}^{n}$ (see Lemma 2 and 3 in [V2]). As in this case $\hat{N} \cong \mathbb{R}^{n} \times \mathrm{G}_{m}\left(\mathbb{R}^{n}\right)$, it was very plain that his Gauß maps take their values in $\mathrm{G}_{m}\left(\mathbb{R}^{n}\right)$ only. Another generalization of Vilms' results was given by H. NAITOH in [Na2]; but it is only valid for symmetric submanifolds in symmetric spaces; the target space of his Gauß map is a symmetric space, whose construction is rather complicated.

(b) Since the tangent spaces of all submanifold of $\mathbb{R}^{n}$ are curvature invariant, Corollary 3 can be interpreted as another generalization of the results given in [V2].

(c) In order not to overload formulas by many $f_{*}$ 's we have formulated them for submanifolds only instead for isometric immersions. It will not be difficult for the reader to reformulate them for an isometric immersion $f: M \rightarrow N$. It is necessary then to replace the second fundamental form $h^{f}$ by the section $\hat{h}^{f}$ in $\mathbb{B}$ along the Gauß map $\xi^{f}: p \mapsto f_{*} T_{p} M$ characterized by

$$
\hat{h}_{p}^{f}\left(f_{*} v, f_{*} w\right)=h_{p}^{f}(v, w) \quad \text { for all } \quad v, w \in T_{p} M(p \in M) .
$$

For instance, by means of (81) formula (120) becomes

$$
h_{p}^{\xi}(u, v)=\Theta_{\xi(p)}\left(A_{f_{*} v}^{b}\right) \quad \text { with } \quad b:=\nabla_{u}^{\mathbb{B}} \hat{h}^{f}
$$

the element $b \in \mathbb{B}_{\xi(p)}$ is also characterized by $b\left(f_{*} v, f_{*} w\right)=\left(\bar{\nabla}_{u} h_{p}^{f}\right)(v, w)$ for $v, w \in T_{p} M$. 


\section{$7 \quad$ Distinguished $m$-planes of $T \hat{N}$}

If $M$ is an $m$-dimensional II-parallel submanifold of $N$, then we learned from Corollary 10 that $\hat{M}:=\xi^{M}(M)$ is an $m$-dimensional affine (=autoparallel) submanifold of $\hat{N}$. Since in this case $M=\tau(\hat{M})$, it is a good idea to try a construction of II-parallel immersions into $N$ by starting from an $m$-dimensional affine submanifold $\hat{M} \subset \hat{N}$ and considering $f:=\tau \mid \hat{M}$. Of course, in general $f$ will not even be an immersion; but if we suppose an additional condition, then we will succeed (see Theorem 8 ). For formulating this condition we assign $m$-dimensional subspaces $\mathcal{T}_{b} \subset T_{W} \hat{N}$ to all bilinear maps $b \in \mathbb{B}_{W}(W \in \hat{N})$, which play a similar role for $m$-dimensional submanifolds as the subspaces of acceleration vectors $\in T(T N)$ for curves in $N$ (see Theorem 7(a)). The geometric impact of the subspaces $\mathcal{T}_{b}$ will be described in Theorem 7 .

Proposition 13 (Definition of $\mathcal{T}_{\boldsymbol{b}}$ ). For every element $W \in \hat{N}$ and every $b \in \mathbb{B}_{W}$ the set

$$
\mathcal{T}_{b}:=\left\{\hat{v} \in T_{W} \hat{N} \mid v:=\tau_{*} \hat{v} \in W \text { and } A_{v}^{b}=\hat{\nu}(\hat{v})\right\}
$$

is an m-dimensional linear subspace of $T_{W} \hat{N}$, which is mapped isomorphically onto $W$ by $\tau_{*}$. The assignment $b \mapsto \mathcal{T}_{b}$ is injective.

Proof. The graph graph $\left(A^{b}\right)$ of the map $A^{b}: W \rightarrow \mathbb{M}_{W}, v \mapsto A_{v}^{b}$ can be considered as an $m$ dimensional linear subspace of $\mathbb{E}_{W} \oplus \mathbb{M}_{W}$; then $\mathcal{T}_{b}$ is the inverse image of $\operatorname{graph}\left(A^{b}\right)$ in $T_{W} \hat{N}$ with respect to the isomorphism $(\hat{\eta}, \hat{\nu}) \mid T_{W} \hat{N}$ (see Theorem 4) and therefore an $m$-dimensional subspace of $T_{W} \hat{N}$. As $b \mapsto A^{b} \mapsto \operatorname{graph}\left(A^{b}\right)$ is injective, the proof is finished.

Example 10 (The Gauß map of a Gauß map). If $M$ is an $m$-dimensional submanifold of $N$ with Gauß map $\xi^{M}$, then $\xi_{*}^{M} T_{p} M=\mathcal{T}_{h_{p}^{M}}$ holds for every point $p \in M$ according to (79).

Remark 14 (The Tsukada distribution). The distribution $\mathcal{D} \subset T \hat{N}$, defined in Remark 12 and already introduced by K. TsuKADA in the article [Ts], can now be described by

$$
\forall W \in \hat{N}: \quad \mathcal{D}_{W}=\mathcal{T}_{0_{W}} \quad \text { with the zero element } \quad 0_{W} \in \mathbb{B}_{W} .
$$

The other subspaces $\mathcal{T}_{b}\left(b \in \mathbb{B}_{W}\right)$ are obtained by tipping $\mathcal{D}_{W}$ up from his horizontal position, but preserving the condition $\tau_{*} \mathcal{T}_{b}=W$.

Proposition 14 (Invariance properties of $\left.\mathcal{T}_{\boldsymbol{b}}\right)$. For every $W \in \hat{N}_{q}(q \in N)$ and every $b \in \mathbb{B}_{W}$ the subspace $\mathcal{T}_{b}$ has the following properties:

(a) Suppose that $b$ is symmetric. Then $\mathcal{T}_{b}$ is torsion invariant in $(\hat{N}, \hat{\nabla})$ (i.e. $T^{\hat{N}}\left(\mathcal{T}_{b}, \mathcal{T}_{b}\right) \subset$ $\left.\mathcal{T}_{b}\right)$ if and only if $W$ is curvature invariant in $N$; moreover, in this case the torsion already vanishes on $\mathcal{T}_{b}$, i.e. $T^{\hat{N}}\left(\mathcal{T}_{b}, \mathcal{T}_{b}\right)=0$.

(b) Provided that $W$ is curvature invariant in $N$, also $\mathcal{T}_{b}$ is curvature invariant in $(\hat{N}, \hat{\nabla})$ if and only if $b$ is $R_{q}^{N}$-semiparallel (see Definition $1(b)$ ).

Proof. We choose $\hat{u}_{i} \in \mathcal{T}_{b}$, put $u_{i}:=\tau_{*} \hat{u}_{i} \in W$, abbreviate $\hat{T}:=T^{\hat{N}}$ and $\hat{R}:=R^{\hat{N}}$ and calculate by means of Proposition 10 and (66):

$$
\begin{aligned}
& \tau_{*} \hat{T}\left(\hat{u}_{1}, \hat{u}_{2}\right)=A_{u_{2}}^{b}\left(u_{1}\right)-A_{u_{1}}^{b}\left(u_{2}\right)=b\left(u_{2}, u_{1}\right)-b\left(u_{1}, u_{2}\right) \quad \text { and } \\
& v:=\tau_{*} \hat{R}\left(\hat{u}_{1}, \hat{u}_{2}\right) \hat{u}_{3}=R^{\mathbb{E}}\left(\hat{u}_{1}, \hat{u}_{2}\right) u_{3} \in W .
\end{aligned}
$$


Suppose now that $b$ is symmetric. Then $\tau_{*} \hat{T}\left(\hat{u}_{1}, \hat{u}_{2}\right)=0$ by means of (124), and the torsion invariance of $\mathcal{T}_{b}$ is equivalent to $\hat{\nu}\left(\hat{T}\left(\hat{u}_{1}, \hat{u}_{2}\right)\right)=0$; according to (93) it is the same as $R^{N}\left(u_{1}, u_{2}\right) \in \mathbb{H}_{W}$. Thus the torsion invariance of $\mathcal{T}_{b}$ is equivalent to the curvature invariance of $W$. Furthermore, $\tau_{*} \hat{T}\left(\hat{u}_{1}, \hat{u}_{2}\right)=0$ and $\hat{\nu}\left(\hat{T}\left(\hat{u}_{1}, \hat{u}_{2}\right)\right)=0$ imply $\hat{T}\left(\hat{u}_{1}, \hat{u}_{2}\right)=0$. Therefore, the proof of assertion (a) is complete.

Henceforth we assume the curvature invariance of $W$. Then we have $R^{N}\left(u_{1}, u_{2}\right) \in \mathbb{H}_{W}$; hence we obtain from (78)

$$
R^{b}\left(u_{1}, u_{2}\right)=R^{\mathbb{E}}\left(\hat{u}_{1}, \hat{u}_{2}\right)
$$

for the tensor $R^{b}$ associated with the quadruple $\left(T_{q} N, W, b, R_{q}^{N}\right)$ according to (10). Because of (125) and (94) $\hat{R}\left(\hat{u}_{1}, \hat{u}_{2}\right) \hat{u}_{3} \in \mathcal{T}_{b}$ is equivalent to $\left[R^{\mathbb{E}}\left(\hat{u}_{1}, \hat{u}_{2}\right), A_{u_{3}}^{b}\right]=A_{v}^{b}$, that means, to

$$
\forall w \in W: \quad R^{\mathbb{E}}\left(\hat{u}_{1}, \hat{u}_{2}\right) b\left(u_{3}, w\right)-b\left(u_{3}, R^{\mathbb{E}}\left(\hat{u}_{1}, \hat{u}_{2}\right) w\right)=b\left(R^{\mathbb{E}}\left(\hat{u}_{1}, \hat{u}_{2}\right) u_{3}, w\right) .
$$

Substituting (126) we get assertion (b).

Remark 15 (On the van der Waerden-Bortolotti connection). From Remark 8, (126) and (72) we see that $b$ is $R_{q}^{N}$-semiparallel (according to (11)) if and only if $W$ is curvature invariant and $R^{\mathbb{B}}\left(\hat{u}_{1}, \hat{u}_{2}\right) b=0$ for all $\hat{u}_{1}, \hat{u}_{2} \in \mathcal{T}_{b}$. Hence, if the tangent space $T_{p} M$ of an $m$-dimensional submanifold $M \subset N$ is curvature invariant, then the second fundamental form $h_{p}^{M}$ is $R_{q}^{N}$ semiparallel if and only if $R^{\mathbb{B}}\left(\xi_{*}^{M} u_{1}, \xi_{*}^{M} u_{2}\right) h_{q}^{M}=0$ holds for all vectors $u_{1}, u_{2} \in T_{q} M$. We remind the reader also of the equation $\nabla_{v}^{\mathbb{B}} h^{M}=\bar{\nabla}_{v} h^{M}$ (see (81)). These results show that the bundle $\left(\mathbb{B}, \nabla^{\mathbb{B}}\right)$ provides a convincing framework for handling the so called van der Waerden-Bortolotti connection $\bar{\nabla}$ of second fundamental forms (see [Ch] p. 65). For instance, saying that the van der Waerden-Bortolotti connection of $M$ is flat (see [L] p. 801) means $R^{\mathbb{B}}\left(\xi_{*}^{M} T_{p} M, \xi_{*}^{M} T_{p} M\right)=0$ for all $p \in M$.

Proposition 15 ("Parallelity" of the map $\boldsymbol{b} \mapsto \mathcal{T}_{\boldsymbol{b}}$ ). If $h: J \rightarrow \mathbb{B}$ is a parallel section in $\mathbb{B}$ along a curve $\xi: J \rightarrow \hat{N}$, then $t \mapsto \mathcal{T}_{h(t)}$ is a $\hat{\nabla}$-parallel subbundle of $T \hat{N}$ along $\xi$, i.e.,

$$
\left.\mathcal{T}_{h(t)}=\stackrel{t}{\|} \|_{t_{0}} \xi\right)^{\hat{N}}\left(\mathcal{T}_{h\left(t_{0}\right)}\right) \quad \text { for all } \quad t_{0}, t \in J
$$

Proof. Let $\hat{v} \in \mathcal{T}_{h\left(t_{0}\right)}$ be given, let $\hat{X} \in \Gamma_{\xi}(T \hat{N})$ denote the $\hat{\nabla}$-parallel vector field along $\xi$ with $\hat{X}\left(t_{0}\right)=\hat{v}$ and put $X:=\tau_{*} \hat{X}$. According to Proposition $10 X$ resp. $\hat{\nu}(\hat{X})$ are parallel sections in $\mathbb{E}$ resp. $\mathbb{M}$. As $\mathbb{T}$ is a parallel subbundle of $\mathbb{E}$ and $X\left(t_{0}\right)=\tau_{*} \hat{v} \in \xi\left(t_{0}\right)=\pi_{\xi\left(t_{0}\right)}$, we get

$$
X(t) \in \pi_{\xi(t)}=\xi(t) \quad \text { for all } \quad t \in J .
$$

Now, let $Y \in \Gamma_{\xi}(\mathbb{\pi})$ be another $\nabla^{\top}$-parallel section. Then $\zeta_{1}: t \mapsto \hat{\nu}(\hat{X}(t)) Y(t)$ and $\zeta_{2}:$ $t \mapsto A_{X(t)}^{h(t)} Y(t)=h(t)(X(t), Y(t))$ are parallel sections of $\Perp$ according to (71) and (69). Since $\hat{X}\left(t_{0}\right) \in \mathcal{T}_{h\left(t_{0}\right)}$, we have $\zeta_{1}\left(t_{0}\right)=\zeta_{2}\left(t_{0}\right)$ and therefore $\zeta_{1} \equiv \zeta_{2}$. Combining this with (128) we obtain $\hat{X}(t) \in \mathcal{T}_{h(t)}$ for all $t \in J$. Thus (127) is proved. 
Remark 16 (The $\mathbb{P}$-morphism $\mathcal{T}: \mathbb{B} \rightarrow \hat{\hat{N}}$ ). As the tangent bundle $T \hat{N}$ is associated with $\mathbb{P}$ (see the construction of $\hat{\nabla}$ ) also the Graßmann bundle $\hat{\tau}: \hat{\hat{N}} \rightarrow \hat{N}$ over $\hat{N}$ with the fibres $\hat{\hat{N}}_{W}=\mathrm{G}_{m}\left(T_{W} \hat{N}\right)$ is associated with $\mathbb{P}$. Thus the connection $\mathcal{H}^{\mathbb{P}}$ induces also a connection for this Graßmann bundle; for a curve $\xi: J \rightarrow \hat{N}$ with $0 \in J$ and an element $\hat{W} \in \hat{\hat{N}}_{\xi(0)}$ the horizontal lift $\hat{W}_{\xi}: J \rightarrow \hat{\hat{N}}$ of $\xi$ through $\hat{W}$ is given by $\hat{W}_{\xi}(t):=\underset{0}{(\| \xi)^{\hat{N}}}(\hat{W})$. The map

$$
\mathcal{T}: \mathbb{B} \rightarrow \hat{\hat{N}}, b \mapsto \mathcal{T}_{b}
$$

turns out to be an injective $\mathbb{P}$-morphism; thus Remark 10 implies Proposition 15 immediately. Furthermore, the image $\mathcal{T}(\mathbb{B})$ is an associated subbundle of $\hat{\hat{N}}$, and $\mathcal{T}: \mathbb{B} \rightarrow \mathcal{T}(\mathbb{B})$ is a $\mathbb{P}$ isomorphism. The proof of these assertions is based on Remark 10 applied on the injective $H$-equivariant map

$$
\begin{aligned}
\phi:(\text { typical fibre of } \mathbb{B}) & =\mathrm{L}^{2}\left(V_{0}, V_{0}^{\perp}\right) \stackrel{\phi_{1}}{\rightarrow} \mathrm{L}\left(V_{0}, \mathfrak{m}\right) \stackrel{\phi_{2}}{\rightarrow} \mathrm{G}_{m}\left(V_{0} \oplus \mathfrak{m}\right) \\
& \stackrel{\phi_{3}}{\longrightarrow} \mathrm{G}_{m}\left(\mathbb{R}^{n} \oplus \mathfrak{m}\right)=(\text { typical fibre of } \hat{\hat{N}} \text {, see the construction of } \hat{\nabla}),
\end{aligned}
$$

where $\phi_{1}$ is the canonical isomorphism $b \mapsto\left(A^{b}: x \mapsto A_{x}^{b}\right)$ (see Definition 1(a) and (57)), $\phi_{2}$ the injective map $\ell \mapsto \operatorname{graph}(\ell)$ and $\phi_{3}$ the canonical inclusion induced by $V_{0} \oplus \mathfrak{m} \hookrightarrow \mathbb{R}^{n} \oplus \mathfrak{m} .{ }^{13}$ With the canonical projections pr: $V_{0} \oplus \mathfrak{m} \rightarrow V_{0}$ and $\widehat{\mathrm{pr}}: \mathbb{R}^{n} \oplus \mathfrak{m} \rightarrow \mathbb{R}^{n}$ we obtain $\phi_{2}\left(\mathrm{~L}\left(V_{0}, \mathfrak{m}\right)\right)=$ $\left\{V \in \mathrm{G}_{m}\left(V_{0} \oplus \mathfrak{m}\right) \mid \operatorname{pr}(V)=V_{0}\right\}$ and therefore $\phi\left(\mathrm{L}^{2}\left(V_{0}, V_{0}^{\perp}\right)\right)=\left\{V \in \mathrm{G}_{m}\left(\mathbb{R}^{n} \oplus \mathfrak{m}\right) \mid \widehat{\operatorname{pr}}(V)=\right.$ $\left.V_{0}\right\}$; hence, $\phi_{2}\left(\mathrm{~L}\left(V_{0}, \mathfrak{m}\right)\right)$ is an open subset of $\mathrm{G}_{m}\left(V_{0} \oplus \mathfrak{m}\right)$ (see $[\mathrm{KN}]$ Vol. 2, p. 133) and thus $\phi\left(\mathrm{L}^{2}\left(V_{0}, V_{0}^{\perp}\right)\right)$ a $H$-invariant, embedded submanifold of $\mathrm{G}_{m}\left(\mathbb{R}^{n} \oplus \mathfrak{m}\right)$, which is the typical fibre of $\mathcal{T}(\mathbb{B})$. Of course, $\mathcal{T}$ turns out to be the $\mathbb{P}$-morphism $\Phi$ associated with $\phi$ according to Remark 10.

In $[\mathrm{A}] \mathrm{W}$. Ambrose has considered the subspaces $\hat{W} \in \hat{\hat{N}}_{W}$, which occur as image $\xi_{*}^{M}\left(T_{p} M\right)$ under the Gauß map $\xi^{M}$ of $m$-dimensional submanifolds $M \subset N$. We denote the set of these subspaces by $\mathcal{E}_{W}^{0}$. Following his ideas we also introduce the subsets

$$
\mathcal{E}_{W}:=\left\{\hat{W} \in \hat{\hat{N}}_{W} \mid \tau_{*} \hat{W}=W\right\} \quad \text { for all } \quad W \in \hat{N} .
$$

We obtain immediately

$$
\mathcal{E}_{W}^{0} \subset \mathcal{E}_{W}
$$

Ambrose calls $\mathcal{E}:=\bigcup \mathcal{E}_{W}$ the bundle of second order m-planes and the elements of $\mathcal{E}^{0}:=\bigcup \mathcal{E}_{W}^{0}$ integrable second order m-planes. Furthermore, for every $p \in N$ and $W \in \hat{N}_{p}$ let $\Gamma_{(p, W)}(\tau)$ denote the set of all sections $\xi: U \rightarrow \hat{N}$ of $\tau$ with $\xi(p)=W$, which are defined on some neighbourhood $U \subset N$ of $p$. Obviously we have

$$
\forall \xi \in \Gamma_{(p, W)}(\tau): \quad \xi_{*}(W) \in \mathcal{E}_{W} .
$$

Considering a section $(\xi: U \rightarrow \hat{N}) \in \Gamma_{(p, W)}(\tau)$ as a distribution on $U$ it make sense to say that it is involutive at $p$; that means: for all vector fields $X, Y \in \Gamma(\xi) \subset \Gamma(T U)$ we have $[X, Y]_{p} \in W$. - Notice that the objects $\mathcal{E}_{W}^{0}, \mathcal{E}_{W}, \Gamma_{(p, W)}(\tau)$ and the involutivity of sections $\xi \in \Gamma_{(p, W)}(\tau)$ are defined without taking account of $\mathbb{B}$ and the metric structure of $N$.

\footnotetext{
${ }^{13}$ Compare this construction with the proof of Proposition 13.
} 
Theorem 7 (The geometric impact of the subspaces $\mathcal{T}_{\boldsymbol{b}}$ ). For every $p \in N$ and $W \in \hat{N}_{p}$ we have:

(a) $\mathcal{E}_{W}=\mathcal{T}\left(\mathbb{B}_{W}\right)$; therefore, $\mathcal{E}$ in fact is a subbundle of $\hat{\tau}: \hat{\hat{N}} \rightarrow \hat{N}$.

(b) $\mathcal{E}_{W}^{0}=\mathcal{T}\left(\left\{b \in \mathbb{B}_{W} \mid b\right.\right.$ is symmetric $\left.\}\right)$; therefore, $\mathcal{E}^{0}$ in fact is a subbundle of $\mathcal{E}$.

(c) $\mathcal{E}_{W}=\left\{\xi_{*}(W) \mid \xi \in \Gamma_{(p, W)}(\tau)\right\}$; moreover, if a local section $\xi \in \Gamma_{(p, W)}(\tau)$ and a map $b \in \mathbb{B}_{W}$ are related by $\xi_{*}(W)=\mathcal{T}_{b}$, then we have ${ }^{14}$

$$
\forall u \in W, Y \in \Gamma(\xi): \quad W^{\perp} \text {-part of } \nabla^{N}{ }_{u} Y=b\left(u, Y_{p}\right) \text {. }
$$

(d) If $\xi \in \Gamma_{(p, W)}(\tau)$ and $b \in \mathbb{B}_{W}$ are related by $\xi_{*}(W)=\mathcal{T}_{b}$, then $\xi$ is involutive at $p$ if and only if $b$ is symmetric.

Proof. For (a). The inclusion $\mathcal{T}_{b} \in \mathcal{E}_{W}$ for $b \in \mathbb{B}_{W}$ is obvious according to Proposition 13. If on the other hand $\hat{W} \in \mathcal{E}_{W}$ is given, let us define $\lambda:=\hat{\nu}_{W} \circ\left(\tau_{*} \mid \hat{W}\right)^{-1} \in \mathrm{L}\left(W, \mathbb{H}_{W}\right)$ and $b \in \mathbb{B}_{W}$ by $b(u, v):=\lambda(u) v$. Then it is easy to prove $\hat{W} \subset \mathcal{T}_{b}$; because $\operatorname{dim} \hat{W}=m=\operatorname{dim} \mathcal{T}_{b}$ we even have $\hat{W}=\mathcal{T}_{b}$.

For (b). In Proposition 8 we have learned that all symmetric bilinear maps $b \in \mathbb{B}_{W}$ occur as second fundamental forms $h_{p:=\tau(W)}^{M}$ of some $m$-dimensional submanifolds $M \subset N$. Therefore Example 10 proves (b).

For (c). The inclusion $\left\{\xi_{*}(W) \mid \xi \in \Gamma_{(p, W)}(\tau)\right\} \subset \mathcal{E}_{W}$ was already noticed in (129). Conversely, for a given subspace $\hat{W} \in \mathcal{E}_{W}$ we must show that there exists a section $\xi \in \Gamma_{(p, W)}(\tau)$ with $\xi_{*}(W)=\hat{W}$. As $\tau$ is a submersion, we can find coordinate systems $x$ resp. $y$ of $\hat{N}$ resp. $N$ about $W$ resp. $p$ with $x(W)=0$ and $y(p)=0$ such that in these coordinates $\tau$ is represented by the canonical projection $p r: \mathbb{R}^{n+m(n-m)} \cong \mathbb{R}^{n} \times \mathbb{R}^{m(n-m)} \rightarrow \mathbb{R}^{n}$. If $\hat{M}$ resp. $M$ are the $m$ dimensional linear subspaces of $\mathbb{R}^{n+m(n-m)}$ resp. $\mathbb{R}^{n}$, whose tangent planes at 0 represent $\hat{W}$ resp. $W$ with respect to the coordinates, then $\tau_{*} \hat{W}=W$ implies that $p r \mid \hat{M}$ is an isomorphism $\hat{M} \rightarrow M$. Now enlarge $\hat{M}$ to an $n$-dimensional subspace $\hat{L}$ of $\mathbb{R}^{n+m(n-m)}$ such that $p r \mid \hat{L}$ also is an isomorphism $\hat{L} \rightarrow \mathbb{R}^{n}$. Then the linear map $\mathbb{R}^{n} \rightarrow \mathbb{R}^{n+m(n-m)}, u \mapsto(p r \mid \hat{L})^{-1}(u)$ is the coordinate representation of a section $\xi \in \Gamma_{(p, W)}(\tau)$ with $\xi_{*}(W)=\hat{W}$.

In order to prove $(130)$ let $(\xi: U \rightarrow \hat{N}) \in \Gamma_{(p, W)}(\tau), b \in \mathbb{B}_{W}$ with $\xi_{*}(W)=\mathcal{T}_{b}, u \in W$ and $Y \in \Gamma(\xi)$ be given and let us define the section $Z: \tau^{-1}(U) \rightarrow \mathbb{E}, V \mapsto\left(V, Y_{\tau(V)}\right)$. Then we have

$$
Z \circ \xi(q)=(\xi(q), Y(q)) \in \pi_{\xi(q)} \quad \text { for all } \quad q \in U,
$$

because $Y$ is a section in $\xi$. Now using $\xi_{*}(W)=\mathcal{T}_{b}$ we calculate by means of Formula (74)

$$
\nabla_{u}^{N} Y=\nabla_{u}^{N}(Z \circ \xi)=\nabla_{\xi_{*} u}^{N} Z=\nabla_{\xi_{*} u}^{\mathbb{E}} Z+\hat{\nu}\left(\xi_{*} u\right) Y_{p}=\nabla_{u}^{\mathbb{E}}(Z \circ \xi)+b\left(u, Y_{p}\right) .
$$

On the one hand (131) implies $\nabla^{\mathbb{E}}(Z \circ \xi) \in \xi(p)=W$, because $\pi$ is a parallel subbundle of $\left(\mathbb{E}, \nabla^{\mathbb{E}}\right)$; on the other hand we have $b\left(u, Y_{p}\right) \in W^{\perp}$; thus Formula (130) follows.

\footnotetext{
${ }^{14}$ Formula (130) shows that $b$ plays a similar role for $\xi$ as the second fundamental form for a submanifold; see also Definition 10(b).
} 
For (d). Let $\xi \in \Gamma_{(p, W)}(\tau)$ and $b \in \mathbb{B}_{W}$ with $\xi_{*}(W)=\mathcal{T}_{b}$ be given. For arbitrary sections $X, Y \in \Gamma(\xi)$ we have $[X, Y]_{p}=\nabla^{N} X_{p} Y-\nabla^{N} Y_{p} X$; therefore (130) implies

$$
W^{\perp} \text {-part of }[X, Y]_{p}=b\left(X_{p}, Y_{p}\right)-b\left(Y_{p}, X_{p}\right) ;
$$

and because of $\xi(p)=W$ the symmetry of $b$ is equivalent to

$$
\forall X, Y \in \Gamma(\xi): \quad[X, Y]_{p} \in W
$$

that means, to the involutivity of $\xi$ at $p$.

As an application of Theorem 7 we derive how the involutivity at a point $p$ of an $m$-dimensional distribution $\xi$ on a manifold $N$ can be read off the second order $m$-plane $\xi_{*}(\xi(p))$ :

Corollary 11 (A criterion for the involutivity of a distribution). A distribution $\xi$ on a differentiable manifold $N$ is involutive at a point $p \in N$ if and only if $\xi_{*}(\xi(p)) \in \mathcal{E}_{\xi(p)}^{0}$, that means, if and only if there exists a submanifold $M \subset N$ with $p \in M, T_{p} M=\xi(p)$ and $\xi^{M}\left(T_{p} M\right)=\xi_{*}(\xi(p))$.

Proof. We choose a riemannian metric on $N$ so that we can apply Theorem 7 , where $m$ is the dimension of the distribution $\xi$. If for a given point $p \in N$ we put $W:=\xi(p)$, then we have $\xi \in \Gamma_{(p, W)}(\tau)$. Now we apply (129) and the assertions (a), (d) and (b) of Theorem 7 .

At the end of the discussion of the bundle $\mathcal{E}$ I will mention another characterization of the subbundle $\mathcal{E}^{0}$, which was given by AMBrose in $[\mathrm{A}]$. For that we consider the subbundle $\hat{\eta}^{-1}(\mathbb{T})=\mathcal{D} \oplus \hat{\mathcal{V}} \subset T \hat{N}$, where $\hat{\eta}: T \hat{N} \rightarrow \mathbb{E}$ is the vector bundle morphism described in Theorem 4, and its orthogonal complement $\hat{\eta}^{-1}(\pi)^{\perp}$ in the cotangent bundle $T^{*} \hat{N}$ with respect to the canonical pairing $T^{*} \hat{N} \times{ }_{\hat{N}} T \hat{N} \rightarrow \mathbb{R},(\hat{\alpha}, \hat{v}) \mapsto \hat{\alpha}(\hat{v})$, that means:

$$
\hat{\eta}^{-1}(\mathbb{\pi})_{V}^{\perp}=\left\{\hat{\alpha} \in T_{V}^{*} \hat{N} \mid \forall \hat{v} \in \hat{\eta}^{-1}(\mathbb{T})_{V}: \hat{\alpha}(\hat{v})=0\right\} \quad \text { for all } \quad V \in \hat{N} .
$$

The global sections of $\hat{\eta}^{-1}(\mathbb{T})^{\perp}$ (considered as differential 1-form on $\hat{N}$ ) are called lift forms by Ambrose. From the definitions we obtain immediately for every local section $\xi: U \rightarrow \hat{N}$ of the bundle $\tau$

$$
\forall q \in U: \hat{W}_{q}:=\xi_{*}(\xi(q)) \subset \hat{\eta}^{-1}(\pi)_{\xi(q)} \text {, hence } \hat{\omega}\left(\hat{W}_{q}\right)=0 \text { for all lift forms } \hat{\omega} \text {. }
$$

By means of Theorem 7 we can now give a rather short proof of the following result:

Proposition 16 (Ambrose, 1964). A second order m-plane $\hat{W} \in \mathcal{E}_{W}$ is integrable (i.e., it belongs to $\left.\mathcal{E}_{W}^{0}\right)$ if and only if $\mathrm{d} \hat{\omega}(\hat{W}, \hat{W})=0$ holds for every lift form $\hat{\omega}$.

Proof. If $p:=\tau(W)$, then according to Theorem 7(c) there exists a local section $(\xi: U \rightarrow \hat{N}) \in$ $\Gamma_{(p, W)}(\tau)$ such that $\hat{W}=\xi_{*}(W)$. Let us first fix a lift form $\hat{\omega}$ and two sections $X, Y \in \Gamma(\xi)$ and consider the 1-form $\omega:=\xi^{*} \hat{\omega}$ on $U$. Because of (132) we have $\omega(X)=\hat{\omega}\left(\xi_{*} X\right) \equiv 0$ and $\omega(Y) \equiv 0$, and therefore

$$
\begin{aligned}
\mathrm{d} \hat{\omega}\left(\xi_{*} X_{p}, \xi_{*} Y_{p}\right) & =\left(\xi^{*} \mathrm{~d} \hat{\omega}\right)\left(X_{p}, Y_{p}\right)=\mathrm{d} \omega\left(X_{p}, Y_{p}\right)=X_{p} \cdot \omega(Y)-Y_{p} \cdot \omega(X)-\omega\left([X, Y]_{p}\right) \\
& =-\hat{\omega}\left(\xi_{*}[X, Y]_{p}\right)
\end{aligned}
$$


Because of $\hat{W}=\xi_{*}(W)$ and since every element $\hat{\alpha} \in \hat{\eta}^{-1}(\mathbb{T})_{W}^{\perp}$ can be extended to a lift form $\hat{\omega}$, the previous formula shows that $\mathrm{d} \hat{\omega}(\hat{W}, \hat{W})=0$ holds for every lift form $\hat{\omega}$ if and only if $\forall X, Y \in \Gamma(\xi): \xi_{*}[X, Y]_{p} \in \hat{\eta}^{-1}(\tau)_{W}$, that means, if and only if $\forall X, Y \in \Gamma(\xi):[X, Y]_{p}=$ $\tau_{*} \xi_{*}[X, Y]_{p} \in W$, whereby the involutivity of $\xi$ at $p$ is characterized. According to Corollary 11 the previous condition is equivalent to $\hat{W} \in \mathcal{E}_{W}^{0}$.

Remark 17 (A further criterion for the involutivity of a distribution). The essential part of the proof of Proposition 16 was to show: A distribution $\xi$ on a differentiable manifold $N$ is involutive at a point $p \in N$ if and only if $\mathrm{d} \hat{\omega}\left(\xi_{*}(\xi(p)), \xi_{*}(\xi(p))\right)=0$ holds for every lift form $\hat{\omega}$.

Theorem 8 (The projection of affine immersions into $\hat{\mathbf{N}}$ ). Let $M$ be a connected $m$ dimensional manifold with a linear connection $\nabla^{M}$ and $\xi: M \rightarrow \hat{N}$ an affine immersion, and assume that there exists a point $p_{0} \in M$ such that $\xi_{*} T_{p_{0}} M \in \mathcal{T}(\mathbb{B})$. Then $f:=\tau \circ \xi$ is an immersion with Gauß map $\xi, \nabla^{M}$ is the Levi-Civita connection of $f^{*}\langle\cdot, \cdot\rangle_{N}$ and in this way $f$ is a II-parallel isometric immersion.

Proof. In a first step we show

$$
\forall p \in M \exists h(p) \in \mathbb{B}_{\xi(p)}: \quad \xi_{*} T_{p} M=\mathcal{T}_{h(p)} .
$$

According to the assumption this assertion is true for $p=p_{0}$. For another point $p \in M$ we choose a curve $c:[0,1] \rightarrow M$ with $c(0)=p_{0}$ and $c(1)=p$ and consider the parallel section $\tilde{h} \in \Gamma_{\xi \circ c}(\mathbb{B})$ with $\tilde{h}(0)=h\left(p_{0}\right)$. Then we know from the affinity of $\xi$ and from Proposition 15 that $t \mapsto \xi_{*} T_{c(t)} M$ and $t \mapsto \mathcal{T}_{\tilde{h}(t)}$ are parallel subbundles of $T \hat{N}$ along $\xi \circ c$ which coincide at $t=0$. Thus we have $\xi_{*} T_{c(t)} M=\mathcal{T}_{\tilde{h}(t)}$ for every $t \in[0,1]$. In particular, (133) is proved also for $p=c(1)$.

Now, according to Proposition 13, $\tau_{*} \mid \mathcal{T}_{h(p)}: \mathcal{T}_{h(p)} \rightarrow \xi(p) \subset T_{f(p)} N$ is injective; hence, $f$ is an immersion and $f_{*} T_{p} M=\tau_{*} \mathcal{T}_{h(p)}=\xi(p)$. Therefore, $\xi$ is the Gauß map of $f$.

Next, for an arbitrary vector field $X \in \Gamma(T M)$ and a vector $v \in T_{p} M$ we calculate

$$
f_{*} \nabla^{M}{ }_{v} X=\tau_{*} \xi_{*} \nabla^{M}{ }_{v} X=\tau_{*} \hat{\nabla}_{v} \xi_{*} X=\nabla^{N} f_{*} X-\hat{\nu}\left(\xi_{*} v\right) f_{*} X_{p}=\nabla^{N} f_{*} X-A_{f_{*} v}^{h(p)} f_{*} X_{p}
$$

the last equality follows from (133). Since $A_{f_{*} v}^{h(p)} f_{*} X_{p} \in \xi(p)^{\perp}=\perp_{p} f, \nabla^{M}$ is the Levi-Civita connection of $f^{*}\langle\cdot, \cdot\rangle_{N}$. Corollary 10 can now be applied: Because $\xi$ is affine, $f$ has parallel second fundamental form.

At the end of this section we introduce prolongations of triples $(q, W, b)$.

Definition 7 (The prolongations of a triple $(\boldsymbol{q}, \boldsymbol{W}, \boldsymbol{b})$ ). Let $c: J \rightarrow N$ be the maximal helical arc generated by a quadruple $(q, W, b, u)$ as described in Definition $3, \xi: J \rightarrow \hat{N}$ the curve associated with the pair $\left(c, A:=A_{u}^{b}\right)$ (according to Lemma 5) and $h: J \rightarrow \mathbb{B}$ the parallel section in $\mathbb{B}$ along $\xi$ with $h(0)=b$. Then we will call $(c, \xi, h)$ the prolongation of the triple $(q, W, b)$ in direction $u$. 
Remark 18. Notice that in the situation of Definition 7 we have $c=\tau \circ \xi, \xi(t)=\varphi_{u}^{b}(t)(W)$ and that $h$ coincides with the section $b_{\xi}$ determined by Formula (68) with $\varphi(t)=\varphi_{u}^{b}(t):=$ $\stackrel{t}{(\| c})^{N} \circ \operatorname{Exp}\left(t A_{u}^{b}\right)$.

Proposition 17 (The geometry of a prolongation of a triple $(\boldsymbol{q}, \boldsymbol{W}, \boldsymbol{b})$ ). In the situation of Definition 7 the curve $\xi$ is the maximal geodesic of $\hat{N}$ with $\tau_{*} \dot{\xi}(0)=u$ and $\hat{\nu}(\dot{\xi}(0))=A_{u}^{b}$, and $t \mapsto \mathcal{T}_{h(t)}$ is a parallel strip along the curve $\xi$, that means, it is a parallel subbundle of $T \hat{N}$ along $\xi$ with $\dot{\xi}(t) \in \mathcal{T}_{h(t)}$ for all $t \in J$ (see Definition 10(a) in Section 10).

Proof. Apply Remark 18, Proposition 11 and 15. Furthermore, notice that $\dot{\xi}(0) \in \mathcal{T}_{b}$ follows from $\tau_{*} \dot{\xi}(0)=u \in W$ and $\hat{\nu}(\dot{\xi}(0))=A_{u}^{b}$ and that therefrom we obtain $\dot{\xi}(t) \in \mathcal{T}_{h(t)}$ because $\dot{\xi}$ is a parallel vector field in $\hat{N}$ and $t \mapsto \mathcal{T}_{h(t)}$ a parallel subbundle of $T \hat{N}$.

Example 11 (Canonical appearance of prolongations). If $M$ is an $m$-dimensional II-parallel submanifold of $N, \tilde{c}: \tilde{J} \rightarrow M$ a unit speed geodesic of $M$ with $p:=\tilde{c}(0)$ and $(c, \xi, h)$ the prolongation of $\left(p, T_{p} M, h_{p}^{M}\right)$ in direction $u:=\dot{\tilde{c}}(0)$ defined over an interval $J$, then Proposition 5(c) and Remark 18 show:

$$
\tilde{J} \subset J, \quad \tilde{c}=c \mid \tilde{J}, \quad T_{\tilde{c}(t)} M=\xi(t) \quad \text { and } \quad h_{\tilde{c}(t)}^{M}=h(t) \quad \text { for all } \quad t \in \tilde{J} .
$$

\section{Proof of Theorem 1}

We assume the situation of Theorem 1 with the notations which were fixed before this theorem, in Proposition 8 and its proof. In particular, for any $u \in W$ let $c_{u}: \tilde{J}_{u} \rightarrow N$ be the maximal helical arc associated with the quadruple $(p, W, b, u)$ and put $\hat{u}:=\left(\tau_{*} \mid \mathcal{T}_{b}\right)^{-1}(u)$ (see Proposition 13). Hence we have

$$
\tau_{*} \hat{u}=u \quad \text { and } \quad \hat{\nu}(\hat{u})=A_{u}^{b} .
$$

From (34) we know

$$
\forall t \in \tilde{J}: \quad\left(t u \in D \quad \text { and } \quad c_{u}(t)=\exp ^{b}(t u)\right) .
$$

Therefore, we can define

$$
\varphi_{u}^{b}(t):=\Phi_{t u}^{b}, \quad \xi_{u}(t):=W_{t u} \quad \text { and } \quad h_{u}(t)=b_{t u} \quad \text { for all } t \in \tilde{J} .
$$

Because of $(33)$ and the definition of $\Phi_{t u}^{b}$ resp. $W_{t u}$ resp. $b_{t u}$ before Theorem 1 we get

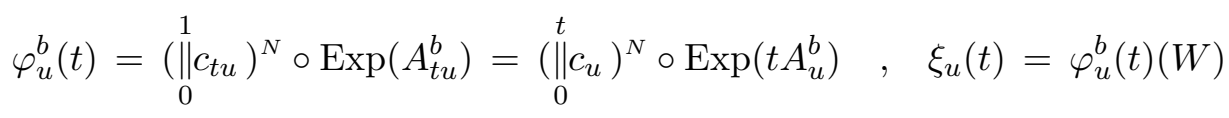

and the following characterization of $h_{u}(t)$ :

$$
h_{u}(t) \circ\left(\varphi_{u}^{b}(t) \times \varphi_{u}^{b}(t)\right)=\varphi_{u}^{b}(t) \circ b .
$$

According to Lemma 5 and the formulae (67), (68) the map $h_{u}: \tilde{J} \rightarrow \mathbb{B}$ is a parallel section along $\xi_{u}$. Therefore, $\left(c_{u}, \xi_{u}, h_{u}\right)$ is the prolongation of $(p, W, b)$ in the direction $u \in W$. Thus we know from Proposition 17 that the curve $\xi_{u}$ is the maximal geodesic of $\hat{N}$ with the initial 
velocity $\dot{\xi}_{u}(0)=\hat{u}$ (because of (134))and the assignment $t \mapsto \mathcal{T}_{h_{u}(t)}$ is a parallel subbundle of $T \hat{N}$ along $\xi_{u}$; in particular we have

$$
\left.\mathcal{T}_{b_{u}}=\underset{0}{\left(\| \xi_{u}\right.}\right)^{\hat{N}}\left(\mathcal{T}_{b}\right)
$$

Moreover, since $\tau_{*}$ gives an isomorphism $\mathcal{T}_{b} \rightarrow W$ by means of Proposition 13, the preimages

$$
\hat{D}:=\left(\tau_{*} \mid \mathcal{T}_{b}\right)^{-1}(D) \quad \text { and } \quad \hat{U}:=\left(\tau_{*} \mid \mathcal{T}_{b}\right)^{-1}(U)
$$

are star shaped neighbourhoods of 0 in $\mathcal{T}_{b}$ with $\hat{U} \subset \hat{D}$. As we have $c_{u}=\tau \circ \xi_{u}$ for all $u \in W$ we get from (135)

$$
\exp ^{b} \circ \tau_{*}\left|\hat{D}=\tau \circ \exp _{W}^{\hat{N}}\right| \hat{D} .
$$

Because $\exp ^{b} \mid U$ is an diffeomorphism onto the helical umbrella $M_{U}(p, W, b) \subset N$, the map $\exp _{W}^{\hat{N}} \mid \hat{U}$ is an injective immersion and therefore $\hat{M}:=\exp _{W}^{\hat{N}}(\hat{U})$ a geodesic umbrella of the "affine" manifold $(\hat{N}, \hat{\nabla})$ with the initial data $\left(W, \mathcal{T}_{b}\right)$; thereby we are reminded of Cartan's theorem from the introduction. Moreover, $\tau \mid \hat{M}$ becomes a diffeomorphism $\hat{M} \rightarrow M_{U}(p, W, b)$.

In [PR1, Theorem 1] was shown that there exists an affine analogon of Cartan's theorem claiming that in an affine manifold such a geodesic umbrella is an affine (=autoparallel) submanifold, if all subspaces $V_{u}$ (in the sense of Cartan's theorem) are torsion and curvature invariant. ${ }^{15} \mathrm{We}$ will now apply this affine analogon using the following lexicon

\begin{tabular}{l||c|c|c|c|c|c|c} 
general analogon & $N$ & $p$ & $V$ & $U$ & $u$ & $c_{u}$ & $V_{u}$ \\
\hline present specification & $\hat{N}$ & $W$ & $\mathcal{T}_{b}$ & $\hat{U}$ & $\hat{u}$ & $\xi_{u}$ & $\mathcal{T}_{b_{u}}$
\end{tabular}.

By means of Proposition 14 the conditions (CI) and (SP) of Theorem 1 imply that the subspaces $\mathcal{T}_{b_{u}} \subset T_{W_{u}} \hat{N}$ are torsion and curvature invariant in the present situation; notice also (136). Thus we obtain from the affine analogon of Cartan's theorem that the geodesic umbrella $\hat{M}$ is an affine submanifold of $\hat{N}$ with $W \in \hat{M}$ and $T_{W} \hat{M}=\mathcal{T}_{b}$. In a last step we apply Theorem 8 to the inclusion $\hat{M} \hookrightarrow \hat{N}$, which is an affine immersion. We obtain that $\tau \mid \hat{M}$ is an injective IIparallel isometric immersion into $N$, and therefore its image $\tau(\hat{M})=M_{U}(p, W, b)$ is a II-parallel submanifold of $N$.

\section{$9 \quad$ A globalization of Theorem 1}

Definition 8 (The saturated subsets $\mathcal{B}\left(\boldsymbol{q}_{\mathbf{0}}, \boldsymbol{W}_{\mathbf{0}}, \boldsymbol{b}_{\mathbf{0}}\right)$ ). We put $\mathcal{B}_{N}:=\{(q, W, b) \mid q \in N, W \in$ $\hat{N}_{q}, b \in \mathbb{B}_{W}$ symmetric $\}$ and say that a subset $\mathcal{B} \subset \mathcal{B}_{N}$ is saturated, if it is not empty and if for every triple $(q, W, b) \in \mathcal{B}$ also the triples $(c(t), \xi(t), h(t))$ of the prolongations of $(q, W, b)$ in the different directions $u \in W$ belong to $\mathcal{B}$ (see Definition 7 ). For every $\left(q_{0}, W_{0}, b_{0}\right) \in \mathcal{B}_{N}$ there exists a smallest saturated subset $\mathcal{B} \subset \mathcal{B}_{N}$ containing $\left(q_{0}, W_{0}, b_{0}\right)$, which will be denoted by $\mathcal{B}\left(q_{0}, W_{0}, b_{0}\right)$ in the following.

\footnotetext{
${ }^{15}$ To be correct, in this Theorem 1 the starting point is a normal neighborhood exp : $U^{T} \rightarrow N$ of the initial point and $U$ is the intersection $U^{T} \cap V$. But it is easy to see that the result keeps valid under the weaker hypothesis on $U$.
} 
Notice that $\mathcal{B}_{N}$ is saturated (since the maps $h(t) \in \mathbb{B}_{\xi(t)}$ from the preceding definition automatically are symmetric) and that the intersection of all saturated subsets $\mathcal{B} \subset \mathcal{B}_{N}$ containing $\left(q_{0}, W_{0}, b_{0}\right)$ is saturated again, whereby the existence of $\mathcal{B}\left(q_{0}, W_{0}, b_{0}\right)$ is proved.

Example 12. If $N$ is a space of constant curvature, then the subset $\mathcal{B}_{0}:=\{(q, W, b) \in$ $\mathcal{B}_{N} \mid b$ is $R_{q}^{N}$-semiparallel $\}$ is saturated, and therefore we have $\mathcal{B}\left(q_{0}, W_{0}, b_{0}\right) \subset \mathcal{B}_{0}$ for every triple $\left(q_{0}, W_{0}, b_{0}\right) \in \mathcal{B}_{0}$. In fact, let $(c, \xi, h)$ be the prolongation of a triple $(q, W, b) \in \mathcal{B}_{0}$ in some direction $u \in W$. Because of Remark 18 and of the special structure of the curvature tensor of $N$ the isometry $\left.\left.\varphi_{u}^{b}(t)=\underset{0}{\left(\|_{0}^{t}\right.}\right)^{N} \circ \underset{\operatorname{Exp}}{(t} A_{u}^{b}\right): T_{q} N \rightarrow T_{c(t)} N$ gives an "isomorphism" between the quadruples $\left(T_{q} N, W, b, R_{q}^{N}\right)$ and $\left(T_{c(t)} N, \xi(t), h(t), R_{c(t)}^{N}\right)$ in the sense of Lemma 2; thus this lemma proves $(c(t), \xi(t), h(t)) \in \mathcal{B}_{0}$.

Definition 9 (Geodesically closed immersions). An affine immersion $f: M \rightarrow N$ into an affine manifold $(N, \nabla)$ resp. a II-parallel isometric immersion $f: M \rightarrow N$ into a riemannian manifold $N$ is said to be geodesically closed, if the image $f \circ c$ of every maximal geodesic $c: J \rightarrow M$ is

(a) a maximal geodesic of $N$ in case that $f$ is affine, and

(b) a maximal helical arc of $N$ in case that $f$ is II-parallel.

Remark 19. Provided that $N$ is geodesically complete, in both cases of Definition 9 such a map $f$ is geodesically closed if and only if $M$ is geodesically complete (take notice of Remark 1(b)).

For later use (see the proof of Theorem 12) we give some information on geodesically closed affine submanifolds:

Lemma 8. For an affine submanifold $M$ of an affine manifold $(N, \nabla)$ the following is true:

(a) If $M$ is a closed, regular submanifold of $N$, then $M$ is geodesically closed; this means that the immersion $M \hookrightarrow N$ is geodesically closed.

(b) If $M$ is geodesically closed and $f: L \rightarrow N$ is an affine immersion from another connected affine manifold $L$ satisfying $f(p) \in M$ and $f_{*} T_{p} L \subset T_{f(p)} M$ for some point $p \in L$, then $f$ is a differentiable map into $M$.

Proof. For (a). Suppose that there exists a maximal geodesic $c: J \rightarrow M$, which in $N$ can be extended to a geodesic $\tilde{c}: \tilde{J} \rightarrow N$ with $J \subsetneq \tilde{J}$; assume for instance $\delta:=\sup J<\sup \tilde{J}$. Since $M$ is closed, we have $p:=\tilde{c}(\delta) \in M$; and since the topology of $M$ is generated by the topology of $N$, the points $c(t)$ converge to $p$ for $t \rightarrow \delta$ with respect to the topology of $M$. But this behaviour is impossible for maximal geodesics of $M$, as follows from the existence of convex neighbourhoods of $p$ in $M .{ }^{16}$

For (b). We define the non-empty subset

$$
L_{0}:=\left\{q \in L \mid f(q) \in M \text { and } f_{*} T_{q} L \subset T_{f(q)} M\right\}
$$

\footnotetext{
${ }^{16}$ By a convex neighbourhood in $M$ we mean a non-empty, open subset of $M$, which is a normal neighbourhood of each of its points; see Theorem 6.2 on p. 34 in [He].
} 
and prove:

If $c: J \rightarrow L$ is a geodesic in $L$ with $0 \in J$ and $q:=c(0) \in L_{0}$, then $f \circ c$ is a geodesic in $M$ and $c(J) \subset L_{0}$.

Since $f_{*} \dot{c}(0) \in T_{f(q)} M$, we can define the maximal geodesic $\tilde{c}: \tilde{J} \rightarrow M$ with $\dot{\tilde{c}}(0)=f_{*} \dot{c}(0)$. As $M$ is a geodesically closed affine submanifold of $N, \tilde{c}$ also is a maximal geodesic in $N$. On the other hand $f \circ c$ is a geodesic in $N$ with the same velocity vector at $t=0$. Therefore we get $f \circ c=\tilde{c} \mid J$; in particular, $f \circ c$ is a geodesic in $M$. Moreover, as $f$ is an affine immersion into $N$ and $M$ is an affine submanifold of $N$, the two subbundles $t \mapsto f_{*} T_{c(t)} L$ and $J \ni t \mapsto T_{\tilde{c}(t)} M$ of $T N$ along the curve $\tilde{c} \mid J$ are parallel. From $f_{*} T_{q} L \subset T_{f(q)} M$ we therefore obtain $f_{*} T_{c(t)} L \subset T_{f(c(t))} M$ for all $t \in J$. Thus (137) is proved. - Using the exponential map of $L$ and $M$ we obtain immediately from (137):

If $U$ is a normal neighbourhood of $q \in L_{0}$ in $L$, then $U \subset L_{0}$ and $f \mid U$ is a $\mathrm{C}^{\infty}$ map into $M$.

Next we prove that

The closur $\overline{L_{0}}$ of $L_{0}$ is contained in the interior $\left(L_{0}\right)^{o}$ of $L_{0}$.

For that let a point $\tilde{q} \in \overline{L_{0}}$ be given. If $U$ is a convex neighbourhood of $\tilde{q}$ in $L$ (see footnote 16), then there exists a point $q \in U \cap L_{0}$. Since $U$ is a normal neighbourhood of $q$, we can apply (138) and find $\tilde{q} \in\left(L_{0}\right)^{\circ}$.

As $L$ was supposed as connected, the statement (139) implies $L=L_{0}$. Therefore, assertion (b) of the Lemma follows from (138).

With Definition 9 at hand we can state:

Theorem 9 (A globalization of the main theorem). Let a triple $\left(q_{0}, W_{0}, b_{0}\right) \in \mathcal{B}_{N}$ be given and assume that for every triple $(q, W, b) \in \mathcal{B}\left(q_{0}, W_{0}, b_{0}\right)$ the subspace $W \subset T_{q} N$ is curvature invariant and the bilinear map $b$ is $R_{q}^{N}$-semiparallel. Then there exists a geodesically closed IIparallel isometric immersion $f: M \rightarrow N$ from an m-dimensional simply connected riemannian manifold $M$ and a point $p_{0} \in M$ such that

$$
f\left(p_{0}\right)=q_{0}, \quad f_{*} T_{p_{0}} M=W_{0} \quad \text { and } \quad h_{p_{0}}^{f}(u, v)=b_{0}\left(f_{*} u, f_{*} v\right) \text { for all } u, v \in T_{p_{0}} M,
$$

and this II-parallel isometric immersion is unique up to isometries, i.e.: If $\tilde{f}: \tilde{M} \rightarrow N$ and $\tilde{p}_{0} \in \tilde{M}$ also have these properties, then there exists an isometry $F: M \rightarrow \tilde{M}$ with $F\left(p_{0}\right)=\tilde{p}_{0}$ and $\tilde{f} \circ F=f$. Furthermore, if $N$ is complete, then also $M$ is complete.

Addendum. By means of Example 12 for a space $N$ of constant curvature the hypothesis on the triple $\left(q_{0}, W_{0}, b_{0}\right)$ can be replaced by the simple assumption that $b_{0}$ is $R_{q_{0}}^{N}$-semiparallel.

We shall deduce this result from [PR1, Theorem 3] which is concerned with the analogous global problem for affine immersions into an affine manifold $(\hat{N}, \hat{\nabla})$. To formulate this result in a suitable manner we introduce the Graßmann bundle $\hat{\tau}: \hat{\hat{N}} \rightarrow \hat{N}$ with the fibre $\mathrm{G}_{m}\left(T_{\hat{q}} \hat{N}\right)$ over $\hat{q} \in \hat{N}$. We say that a subset $\mathcal{A} \subset \hat{\hat{N}}$ is saturated, if it is not empty and if for every subset $\hat{W} \in \mathcal{A}$ and for every geodesic $\gamma: J \rightarrow \hat{N}$ with $\gamma(0)=\hat{\tau}(\hat{W})$ and $\dot{\gamma}(0) \in \hat{W}$ and for every 
$t \in J$ also the parallel translate $\left.\stackrel{t}{\|}_{0}^{t}\right)^{\hat{N}}(\hat{W})$ belongs to $\mathcal{A}$. For every $\hat{W}_{0} \in \hat{\hat{N}}$ there exists a smallest saturated subset $\mathcal{A} \subset \hat{\hat{N}}$ containing $\hat{W}_{0}$, which will be denoted by $\mathcal{A}\left(\hat{W}_{0}\right)$. Now the cited result says:

Theorem ([PR1, Theorem 3]). Let a subspace $\hat{W}_{0} \in \hat{\hat{N}}_{\hat{q}_{0}}\left(\hat{q}_{0} \in \hat{N}\right)$ be given and assume that every subspace $\hat{W} \in \mathcal{A}\left(\hat{W}_{0}\right)$ is torsion and curvature invariant. Then there exists a geodesically closed affine immersion $f: M \rightarrow \hat{N}$ from an m-dimensional simply connected affine manifold $\left(M, \nabla^{M}\right)$ and a point $p_{0} \in M$ such that

$$
f\left(p_{0}\right)=\hat{q}_{0} \quad \text { and } \quad f_{*} T_{p_{0}} M=\hat{W}_{0}
$$

and this immersion is unique up to affine diffeomorphisms. Furthermore, if $\hat{N}$ is geodesically complete, then also $M$ is geodesically complete.

In [PR1] the set $\mathcal{A}\left(\hat{W}_{0}\right)$ was not introduced, but the hypothesis of Theorem 3 was formulated equivalently by means of distinguished broken geodesics. Analogously the hypothesis of Theorem 9 can be formulated by means of broken helical curves; but we hesitate to do so, because it is rather lengthy (some impression can be obtained in the proof of Theorem 10).

Proof of Theorem 9. Let $(\hat{N}, \hat{\nabla})$ have the special meaning of this thesis; then the fibre bundle $\hat{\tau}: \hat{\hat{N}} \rightarrow \hat{N}$ already appeared in Remark 16. We introduce the map

$$
\chi: \mathcal{B}_{N} \rightarrow \hat{\hat{N}},(q, W, b) \mapsto \mathcal{T}_{b}
$$

and prove:

If $\mathcal{B}$ is a saturated subset of $\mathcal{B}_{N}$, then $\chi(\mathcal{B})$ is a saturated subset of $\hat{\hat{N}}$.

For that let a triple $(q, W, b) \in \mathcal{B}$ and a maximal geodesic $\xi: J \rightarrow \hat{N}$ with $\xi(0)=W$ and $\dot{\xi}(0) \in \mathcal{T}_{b}$ be given. We must show

$$
\underset{0}{\stackrel{t}{\| \xi})^{\hat{N}}\left(\mathcal{T}_{b}\right) \in \chi(\mathcal{B}) \text { for all } \quad t \in J}
$$

For that notice that according to Proposition 11 and Remark 18 the prolongation of $(q, W, b)$ in the direction $u:=\tau_{*} \dot{\xi}(0) \in W$ is $(c, \xi, h)$ with $c:=\tau \circ \xi$ and with the parallel section $h=b_{\xi}: J \rightarrow \mathbb{B}$. Since $\mathcal{B}$ is saturated, also the triples $(c(t), \xi(t), h(t))$ belong to $\mathcal{B}$. From Proposition 17 we therefore obtain:

$$
\left.\underset{0}{t} \|^{t}\right)^{\hat{N}}\left(\mathcal{T}_{b}\right)=\mathcal{T}_{h(t)}=\chi(c(t), \xi(t), h(t)) \in \chi(\mathcal{B}) .
$$

Thus (141) and (140) are verified.

Now, applying $(140)$ to $\mathcal{B}=\mathcal{B}\left(q_{0}, W_{0}, b_{0}\right)$ we get $\mathcal{A}\left(\mathcal{T}_{b_{0}}\right) \subset \chi\left(\mathcal{B}\left(q_{0}, W_{0}, b_{0}\right)\right)$. Moreover, the hypothesis of Theorem 9 implies the hypothesis of the previous theorem for $\hat{W}_{0}:=\mathcal{T}_{b_{0}}$ by means of Proposition 14. Thus there exists a geodesically closed affine immersion $\hat{f}: M \rightarrow \hat{N}$ from an $m$-dimensional simply connected affine manifold $\left(M, \nabla^{M}\right)$ and a point $p_{0} \in M$ such that $\hat{f}\left(p_{0}\right)=W_{0}$ and $\hat{f}_{*} T_{p_{0}} M=\mathcal{T}_{b_{0}}$. According to Theorem 8, the third formula of (79) (take 
also notice of Remark 13(c)) and Example $10 f:=\tau \circ \hat{f}$ gives us the desired II-parallel isometric immersion. It is geodesically closed by means of Proposition 11, because this holds for $\hat{f}$.

The uniqueness of $f$ follows from the uniqueness of $\hat{f}$. Indeed, if $\tilde{f}: \tilde{M} \rightarrow N$ is another "solution" (as described in Theorem 9), then its Gauß map $\tilde{\xi}: \tilde{M} \rightarrow \hat{N}$ is an affine immersion (according to Corollary 10) with $\tilde{\xi}\left(\tilde{p}_{0}\right)=W_{0}$ and $\tilde{\xi}_{*} T_{\tilde{p}_{0}} \tilde{M}=\mathcal{T}_{b_{0}}$ (see Example 10). Furthermore, by means of Proposition $11 \tilde{\xi}$ is geodesically closed (as this holds for $\tilde{f}$ ). Therefore the uniqueness assertion of the previous theorem implies that there exists an affine diffeomorphism $F: M \rightarrow \tilde{M}$ with $F\left(p_{0}\right)=\tilde{p}_{0}$ such that $\tilde{\xi} \circ F=\hat{f}$. Consequently we have $\tilde{f} \circ F=f$; in particular, $F$ is an isometry.

Finally the completeness result follows from Remark 19.

Interpretating (42) in geometric terms we get by means of Lemma 2 and the Remarks 6 and 18: If in a symmetric space $N\left(c_{u}, \xi_{u}, h_{u}\right)$ is the prolongation of a triple $\left(q_{0}, W_{0}, b_{0}\right)$ in some direction $u \in W_{0}$ and if $\xi_{u}(t)$ is curvature invariant and $h_{u}(t) R_{c_{u}(t)}^{N}$-semiparallel for the parameters $t \in J_{u}$, then these "integrability conditions" also are satisfied for all $t \in \mathbb{R}$. The following theorem generalizes this "local $\Rightarrow$ global" principle.

Theorem 10 (Completion of II-parallel submanifolds of symmetric spaces). If $N$ is a riemannian symmetric space, then for every connected II-parallel submanifold $M_{\mathrm{loc}} \subset N$ there exists a II-parallel isometric immersion $f: M \rightarrow N$ from a symmetric space $M$ such that

$$
\tilde{M}:=\left\{p \in M \mid f(p) \in M_{\mathrm{loc}}, f_{*} T_{p} M=T_{f(p)} M_{\mathrm{loc}} \text { and } h_{p}^{f}=f^{*} h_{f(p)}^{M_{\mathrm{loc}}}\right\}
$$

is a non-empty open submanifold of $M$ and $f \mid \tilde{M}$ is an isometric covering onto $M_{\mathrm{loc}}$.

Proof. We fix some point $q_{0} \in M_{\mathrm{loc}}$ and define $W_{0}:=T_{q_{0}} M_{\mathrm{loc}}, b_{0}:=h_{q_{0}}^{M_{\mathrm{loc}}}, \mathcal{B}_{0}\left(q_{0}, W_{0}, b_{0}\right):=$ $\left\{\left(q_{0}, W_{0}, b_{0}\right)\right\}$ and

$$
\begin{aligned}
& \mathcal{B}_{k}\left(q_{0}, W_{0}, b_{0}\right):=\{(c(t), \xi(t), h(t)) \mid t \in \mathbb{R} \text { and }(c, \xi, h) \text { is the prolongation of a triple } \\
& \left.(q, W, b) \in \mathcal{B}_{k-1}\left(q_{0}, W_{0}, b_{0}\right) \text { in direction of some } u \in W\right\} \quad \text { for } \quad k \in \mathbb{N} \text {. }
\end{aligned}
$$

Then we have $\mathcal{B}_{k-1}\left(q_{0}, W_{0}, b_{0}\right) \subset \mathcal{B}_{k}\left(q_{0}, W_{0}, b_{0}\right)$ for all $k \in \mathbb{N}$ and $\mathcal{B}\left(q_{0}, W_{0}, b_{0}\right)=$ $\bigcup_{k \in \mathbb{N}} \mathcal{B}_{k}\left(q_{0}, W_{0}, b_{0}\right)$. Therefore, the hypothesis of Theorem 9 are satisfied if for every $k \in \mathbb{N}$ the following holds:

For every triple $(q, W, b) \in \mathcal{B}_{k}\left(q_{0}, W_{0}, b_{0}\right)$ the subspace $W \subset T_{q} N$ is $R_{q}^{N}$-invariant and the bilinear map $b$ is $R_{q}^{N}$-semiparallel.

Using the notations, which were introduced before Theorem 2, we first will prove that (143) is equivalent to:

For every $k$-tuple $\boldsymbol{u}=\left(u_{1}, \ldots, u_{k}\right)$ of vectors $u_{i} \in W_{0}$ the values of the function

$$
R_{\boldsymbol{u}}: \mathbb{R}^{k} \rightarrow \mathfrak{C}\left(T_{q_{0}} N\right),\left(t_{1}, \ldots, t_{k}\right) \mapsto \operatorname{Exp}\left(t_{k} \hat{A}\left(u_{k}\right)\right) \circ \cdots \circ \operatorname{Exp}\left(t_{1} \hat{A}\left(u_{1}\right)\right) R_{q_{0}}^{N}
$$

with $\hat{A}\left(u_{i}\right):=-\left(A_{u_{i}}^{b_{0}}\right)_{\mathfrak{C}} \in$ End $_{-}\left(\mathfrak{C}\left(T_{q_{0}} N\right)\right)$ lie in $\mathfrak{C}\left(T_{q_{0}} N, W_{0}, b_{0}\right)$.

For $k=1$. A triple $\left(q_{1}, W_{1}, b_{1}\right)$ lies in $\mathcal{B}_{1}\left(q_{0}, W_{0}, b_{0}\right)$ if and only if there exists a prolongation $(c, \xi, h)$ from $\left(q_{0}, W_{0}, b_{0}\right)$ in a direction $u_{1} \in W_{0}$ and a parameter $t \in \mathbb{R}$ such that 
$\left(q_{1}, W_{1}, b_{1}\right)=(c(t), \xi(t), h(t))$. Remark 18, the definition of $R_{u}(t)$ before Theorem 1 and Formula (40) show that the isometry $\left.\varphi_{u_{1}}^{b_{0}}(t):=\stackrel{t}{\| c}\right)_{0}^{N} \circ \operatorname{Exp}\left(t A_{u_{1}}^{b_{0}}\right)$ gives an isomorphism between the quadruples $\left(T_{q_{0}} N, W_{0}, b_{0}, R_{u_{1}}(t)\right)$ with $R_{u_{1}}(t)=\left(\operatorname{Exp}\left(-t A_{u_{1}}^{b_{0}}\right)\right)_{\mathfrak{C}} R_{q_{0}}^{N}=\operatorname{Exp}\left(t \hat{A}\left(u_{1}\right)\right) R_{q_{0}}^{N}$ and $\left(T_{q_{1}} N, W_{1}, b_{1}, R_{q_{1}}^{N}\right)$ in the sense of Lemma 2. Thus this lemma shows the equivalence of (143) and (144) in case $k=1$.

For $k=2$. A triple $(q, W, b)$ lies in $\mathcal{B}_{2}\left(q_{0}, W_{0}, b_{0}\right)$ if and only if there exists a triple $\left(q_{1}, W_{1}, b_{1}\right) \in \mathcal{B}_{1}\left(q_{0}, W_{0}, b_{0}\right)$, a prolongation $(\tilde{c}, \tilde{\xi}, \tilde{h})$ from $\left(q_{1}, W_{1}, b_{1}\right)$ in a direction $v \in W_{1}$ and a parameter $s \in \mathbb{R}$ such that $(q, W, b)=(\tilde{c}(s), \tilde{\xi}(s), \tilde{h}(s))$. As in case $k=1$ the isometry $\varphi_{v}^{b_{1}}(s):=(\stackrel{s}{\| \tilde{c}})^{N} \circ \operatorname{Exp}\left(s A_{v}^{b_{1}}\right)$ gives an isomorphism between the quadruples $\left(T_{q_{1}} N, W_{1}, b_{1}, R_{v}(s)\right)$ with $\left.R_{v}(s)=\operatorname{Exp}\left(-s A_{v}^{b_{1}}\right)\right)_{\mathfrak{C}} R_{q_{1}}^{N}$ and $\left(T_{q} N, W, b, R_{q}^{N}\right)$. For the triple $\left(q_{1}, W_{1}, b_{1}\right) \in \mathcal{B}_{1}$ we use the description $\left(q_{1}, W_{1}, b_{1}\right)=(c(t), \xi(t), h(t))$ of the preceding case. Then there exists a unit vector $u_{2} \in W_{0}$ such that $v=\varphi_{u_{1}}^{b_{0}}(t) u_{2}$. Therefore, because of Remark 18 we get $b_{1}\left(v, \varphi_{u_{1}}^{b_{0}}(t) w\right)=$ $\varphi_{u_{1}}^{b_{0}}(t) b_{0}\left(u_{2}, w\right)$ for every further vector $w \in W_{0}$, hence $A_{v}^{b_{1}} \circ \varphi_{u_{1}}^{b_{0}}(t)=\varphi_{u_{1}}^{b_{0}}(t) \circ A_{u_{2}}^{b_{0}}$ and eventually $\operatorname{Exp}\left(s A_{v}^{b_{1}}\right) \circ \varphi_{u_{1}}^{b_{0}}(t)=\varphi_{u_{1}}^{b_{0}}(t) \circ \operatorname{Exp}\left(s A_{u_{2}}^{b_{0}}\right)$. Now it is not difficult to show that the isometry $\varphi_{u_{1}}^{b_{0}}(t)$ gives an isomorphism between the quadruples $\left(T_{q_{0}} N, W_{0}, b_{0}, R_{\boldsymbol{u}}(t, s)\right)$ with $\boldsymbol{u}:=\left(u_{1}, u_{2}\right)$ and $\left(T_{q_{1}} N, W_{1}, b_{1}, R_{v}(s)\right)$. Thus the isometry $\varphi_{v}^{b_{1}}(s) \circ \varphi_{u_{1}}^{b_{0}}(t)$ gives an isomorphism between the quadruples $\left(T_{q_{0}} N, W_{0}, b_{0}, R_{\boldsymbol{u}}(t, s)\right)$ and $\left(T_{q} N, W, b, R_{q}^{N}\right)$, and Lemma 2 implies the equivalence of (143) and (144) in case $k=2$.

For $k>2$ the equivalence "(143) $\Leftrightarrow(144)$ " is proved by induction using $\mathcal{B}_{k}\left(q_{0}, W_{0}, b_{0}\right)=$ $\cup \mathcal{B}_{k-1}\left(q_{1}, W_{1}, b_{1}\right),\left(q_{1}, W_{1}, b_{1}\right) \in \mathcal{B}_{1}\left(q_{0}, W_{0}, b_{0}\right)$; all essential steps were already carried out in case $k=2$; therefore we do not go into more details.

Let us now show that for every $k \in \mathbb{N}$ and every $k$-tuple $\boldsymbol{u}=\left(u_{1}, \ldots, u_{k}\right)$ of vectors $u_{i} \in W_{0}$

$$
\text { there exists a neighbourhood } G \subset \mathbb{R}^{k} \text { of } 0 \text { with } R_{\boldsymbol{u}}(G) \subset \mathfrak{C}\left(T_{q_{0}} N, W_{0}, b_{0}\right) \text {. }
$$

The construction of the neighbourhood $G$ is somewhat complicated. We construct $\mathrm{C}^{\infty}$-maps $g_{i}: G_{i} \rightarrow$ $M_{\mathrm{loc}}$ for $i=1, \ldots, k$ and vector fields $Y_{i}$ in $M_{\mathrm{loc}}$ along $g_{i}$ for $i=1, \ldots, k-1$ by recursion: $g_{1}$ : $G_{1} \rightarrow M_{\text {loc }}$ is defined as the maximal geodesic of $M_{\text {loc }}$ with $\dot{g}_{1}(0)=u_{1}$; by $X_{12}, \ldots, X_{1 k}$ we denote the parallel vector fields along $g_{1}$ with the initial values $X_{1 i}(0)=u_{i}$, and we put $Y_{1}:=X_{12}$. Then the map $g_{2}: G_{2} \rightarrow M_{\text {loc }}$ is characterized by the fact that $G_{2}$ is a neighbourhood of $G_{1} \times\{0\}$ in $\mathbb{R}^{2}$ and for each $t_{1} \in G_{1}$ the map $t \mapsto g_{2}\left(t_{1}, t\right)$ is the maximal geodesic in $M_{\text {loc }}$ with initial velocity $Y_{1}\left(t_{1}\right)$; furthermore, let $X_{23}, \ldots, X_{2 k}$ be the vector fields along $g_{2}$ which are parallel along the before mentioned geodesics and satisfy $X_{2 i}\left(t_{1}, 0\right)=X_{1 i}\left(t_{1}\right)$ for all $t_{1} \in G_{1}$ and put $Y_{2}:=X_{23}$. Continuing this procedure we construct the further maps $g_{i}: G_{i} \rightarrow M_{\text {loc }}$ and get $G:=G_{k}$ as an open neighbourhood of $G_{k-1} \times\{0\}$ in $\mathbb{R}^{k}$. In order to prove now (145) one considers for every $\left(t_{1}, \ldots, t_{k}\right) \in G$ the broken geodesic which is composed by the geodesic arcs $[0,1] \ni s \mapsto g_{1}\left(s t_{1}\right),[0,1] \ni s \mapsto g_{2}\left(t_{1}, s t_{2}\right), \ldots,[0,1] \ni s \mapsto g_{k}\left(t_{1}, \ldots, t_{k-1}, s t_{k}\right)$; then one obtains $\left(q_{i}, T_{q_{i}} M_{\mathrm{loc}}, h_{q_{i}}^{M_{\mathrm{loc}}}\right) \in \mathcal{B}_{i}\left(q_{0}, W_{0}, b_{0}\right)$ for $q_{i}:=g_{i}\left(t_{1}, \ldots, t_{i}\right)$ and $i=1, \ldots, k$ by means of Proposition 5(c); because of $R_{q}^{N} \in \mathfrak{C}\left(T_{q} N, T_{q} M_{\mathrm{loc}}, h_{q}^{M_{\mathrm{loc}}}\right)$ for all $q \in M$ (see Proposition 5(a) and (b)), one derives $R_{\boldsymbol{u}}\left(t_{1}, \ldots, t_{k}\right) \in \mathfrak{C}\left(T_{q_{0}} N, W_{0}, b_{0}\right)$ with the same arguments as in the proof of "(143) $\Leftrightarrow(144)$ ".

From (145) we get

$$
\begin{aligned}
& R_{q_{0}}^{N} \in \mathfrak{C}\left(T_{q_{0}} N, W_{0}, b_{0}\right) \quad \text { and } \\
& \hat{A}\left(u_{k}\right) \circ \cdots \circ \hat{A}\left(u_{1}\right) R_{q_{0}}^{N}=\frac{\partial^{k} R_{\boldsymbol{u}}}{\partial t_{1} \ldots \partial t_{k}}(0, \ldots, 0) \in \mathfrak{C}\left(T_{q_{0}} N, W_{0}, b_{0}\right)_{L} .
\end{aligned}
$$


In order to prove now the validity of (144) we approximate the exponential maps $\operatorname{Exp}\left(t_{i} \hat{A}\left(u_{i}\right)\right)$ by their partial sums and get the approximations

$$
R_{\boldsymbol{u}, \ell}\left(t_{1}, \ldots, t_{k}\right)=\sum_{i_{1}, \ldots, i_{k}=0}^{\ell} \frac{1}{i_{1} ! \cdots i_{k} !} \cdot t_{1}^{i_{1}} \cdots t_{k}^{i_{k}} \cdot \hat{A}\left(u_{k}\right)^{i_{k}} \circ \cdots \circ \hat{A}\left(u_{1}\right)^{i_{1}} R_{q}^{N} \quad(\ell \in \mathbb{N})
$$

of $R_{\boldsymbol{u}}\left(t_{1}, \ldots, t_{k}\right)$. By means of (146) we obtain $R_{\boldsymbol{u}, \ell}\left(\mathbb{R}^{k}\right) \subset \mathfrak{C}\left(T_{q_{0}} N, W_{0}, b_{0}\right)$ for all $\ell \in \mathbb{N}$ and therefore eventually $R_{\boldsymbol{u}}\left(\mathbb{R}^{k}\right) \subset \mathfrak{C}\left(T_{q_{0}} N, W_{0}, b_{0}\right)$. Thereby (144) is proved.

After that we can apply Theorem 9 (as described in the beginning of this proof) and find that there exists a complete, simply connected riemannian manifold $M$, a point $p_{0} \in M$ and a II-parallel isometric immersion $f: M \rightarrow N$, such that $p_{0} \in \tilde{M}$ and $f\left(p_{0}\right)=q_{0}$, where $\tilde{M}$ is the subset defined in (142). Taking Corollary 2 into account we see that $M$ even is a symmetric space. For every $p \in \tilde{M}$ we can now choose a open neighbourhood $U(p) \subset M$ of $p$ such that $f(U(p))$ is another II-parallel submanifold of $N$. From Corollary 4 we find that $f(U(p))$ is an open submanifold of $M_{\text {loc }}$, if we choose $U(p)$ sufficiently small; obviously, then $f \mid U(p)$ is an isometry into $M_{\text {loc }}$; therefrom we obtain $U(p) \subset \tilde{M}$. Hence, $\tilde{M}$ is an open submanifold of $M$ and $f \mid \tilde{M}$ is a local isometry into $M_{\text {loc }}$. - Next we prove:

The local isometry $f \mid \tilde{M}: \tilde{M} \rightarrow M_{\text {loc }}$ is geodesically closed (in the sense of Definition $9(\mathrm{a}))$.

For (147). Let be given a point $p \in \tilde{M}$ and a vector $\tilde{u} \in T_{p} \tilde{M}$ and let $\tilde{c}: \tilde{J} \rightarrow \tilde{M}$ resp. $c: J \rightarrow M_{\text {loc }}$ be the maximal geodesics of $\tilde{M}$ resp. $M_{\text {loc }}$ with $\dot{\tilde{c}}(0)=\tilde{u} \operatorname{resp.} \dot{c}(0)=f_{*} \tilde{u}$. Obviously we have $\tilde{J} \subset J$ and $c \mid \tilde{J}=f \circ \tilde{c}$, and we must show $\tilde{J}=J$. Because of the completeness of $M$ the geodesic $\tilde{c}$ can be extended to a geodesic $\mathbb{R} \rightarrow M$ of $M$, which we denote by $\tilde{c}$ again. Now we will show $\tilde{c}(J) \subset \tilde{M}$, wherefrom we get $\tilde{J}=J$ immediately: Since $\tilde{M}$ is open in $M$, the set $I:=\{t \in J \mid \tilde{c}(t) \in \tilde{M}\}$ is open in $J$. It suffices now to show that $I$ is

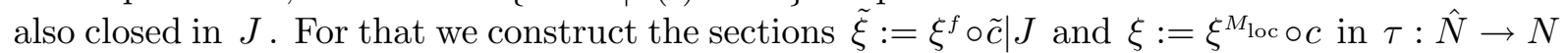
along $\tilde{c} \mid J$ resp. $c$ (with the Gauß maps of $f$ resp. $M_{\text {loc }}$ ) and the sections $\tilde{h}: J \rightarrow \mathbb{B}$ resp. $h: J \rightarrow \mathbb{B}$ of the bundle $\mathbb{B}$ along $\tilde{\xi}$ resp. $\xi$ by means of the second fundamental forms of $f$ resp. $M_{\mathrm{loc}}$ :

$$
f^{*} \tilde{h}(t):=h_{\tilde{c}(t)}^{f} \quad \text { and } \quad h(t)=h_{c(t)}^{M_{\text {loc }}} .
$$

Since these sections are continous and $I=\{t \in J \mid f \circ \tilde{c}(t)=c(t), \tilde{\xi}(t)=\xi(t) \tilde{h}(t)=h(t)\}$ holds, it is obvious that $I$ is closed in $J$. Thus (147) is proved.

Because of (147) the hypotheses of the following lemma are satisfied; therefore, $f \mid \tilde{M}: \tilde{M} \rightarrow M_{\text {loc }}$ is in fact a covering.

Lemma 9. Every local affine diffeomorphism $f: \tilde{M} \rightarrow M$ into a connected affine manifold $\left(M, \nabla^{M}\right)$, which is geodesically closed (in the sense of Definition 9(a)), is a covering.

A special riemannian version of this lemma (assuming $\tilde{M}$ to be complete, instead of $f$ being geodesically closed) is Theorem 4.6(a) in [KN], Vol.1, p. 176, which was generalized to affine manifolds by Hicks (see Theorem 3 in [Hi]). I learned the present version of Lemma 9 in 1998 from H. Reckziegel attending a course on differential geometry; its proof works in the lines of Hicks' argumentation. 


\section{Geometry of strips}

First we suppose that $N$ is only equipped with a linear connection $\nabla^{N}$.

Definition 10 (Strips and second fundamental forms for them). (a) A subbundle $\xi$ of $T N$ along some map $f: M \rightarrow N$ is called a strip in $N$ along $f$ of rank $m$, if $\xi(p)$ is an $m$-dimensional subspace of $T_{f(p)} N$ and $f_{*} T_{p} M \subset \xi(p)$ for all $p \in M$.

(b) If $N$ is a riemannian manifold and $\xi$ a strip in $N$ along $f: M \rightarrow N$ of rank $m$, then a section $h: M \rightarrow \mathbb{B}$ along $\xi$ is called a second fundamental form for $\xi$, if for all vector fields $X \in \Gamma(T M)$ and all sections $Y \in \Gamma(\xi)$ we have

$$
\left(\xi^{\perp} \text {-part of } \nabla_{X}^{N} Y\right)=h\left(f_{*} X, Y\right) .
$$

Notice, $\xi$ can be considered as a map into $\hat{N}$ and the previous equation can be written as $\hat{\nu}\left(\xi_{*} X\right) Y=h\left(f_{*} X, Y\right)$ (see (75) and (76)); thus it is equivalent to

$$
\hat{\nu}\left(\xi_{*} v\right)=A_{f_{*} v}^{h(p)} \text { for all } v \in T_{p} M(p \in M) .
$$

Up to this point only symmetric elements of $\mathbb{B}$ has been used essentially, ${ }^{17}$ but now nonsymmetric elements are usefull to enlarge the range of strips which possess a second fundamental form. Strips are generalizations of immersions $f: M \rightarrow N$ because the Gauß map $\xi^{f}: p \mapsto$ $f_{*} T_{p} M$ is a strip along $f$. In this case the version $\hat{h}^{f}$ of the second fundamental form of $f$ described in Remark 13(b) is the only possible second fundamental form of the strip $\xi^{f}$ (compare (148) with (79)). Already W. Blaschke has used strips along curves (see [BL] p. 80).

Proposition 18 (Characterization of strips with second fundamental form). Let $N$ be a riemannian manifold, for which a map $\xi: M \rightarrow \hat{N}$ and a section $h \in \Gamma_{\xi}(\mathbb{B})$ are given, put $f:=\tau \circ \xi$ and $\hat{\xi}:=\mathcal{T} \circ h$ (see Remark 16).

(a) The following assertions are pairwise equivalent:

(i) $\xi$ is a strip in $N$ along $f$ with second fundamental form $h$.

(ii) $\xi_{*} T_{p} M \subset \mathcal{T}_{h(p)} \quad$ for all $\quad p \in M$

(iii) $\hat{\xi}$ is a strip in $\hat{N}$ along $\xi$.

(b) If $\xi$ is a strip in $N$ along $f$ with second fundamental form $h$, then $h$ is a parallel section of $\mathbb{B}$ if and only if the strip $\hat{\xi}$ is parallel, i.e., if it is a parallel subbundle of $T \hat{N}$.

Assertion (b) is a generalization of Corollary 10.

Proof. The equivalence (i) $\Leftrightarrow$ (ii) of (a) follows immediately from (122) in combination with (148), and the equivalence (ii) $\Leftrightarrow$ (iii) is obvious because of Definition 10(a). Eventually, assertion (b) follows, because $\mathcal{T}$ is a $\mathbb{P}$-isomorphism $\mathbb{B} \rightarrow \mathcal{T}(\mathbb{B})$ (see Remark 10).

\footnotetext{
${ }^{17}$ An exception is the description of the geometric impact of the general subspaces $\mathcal{T}_{b}$ in Theorem 7 .
} 
Because of the previous assertion (b) we are now interested in parallel strips in an affine manifold $N$ equipped only with a connection $\nabla^{N}$. Of course, the Graßmann bundle $\tau: \hat{N} \rightarrow N$ can then also be defined, and $\nabla^{N}$ induces a connection $\hat{\mathcal{H}}$ for this bundle as was described at the beginning of Section 6. In particular, Formula (86) keeps valid. Thus a subbundle $\xi$ of $T N$ along a map $f: M \rightarrow N$ of rank $m$ is parallel if and only if it is a $\hat{\mathcal{H}}$-horizontal map into $\hat{N}$. Furthermore, the Tsukada distribution $\mathcal{D}$ described in Remark 14 can be introduced in this general situation (see [PR1] p. 348); it is valuable for us, because we immediately obtain from (123):

Proposition 19 (Parallel strips and the Tsukada distribution). A strip $\xi$ in $N$ along a map $f: M \rightarrow N$ of rank $m$ is parallel if and only if it is a $\mathcal{D}$-integral map into $\hat{N}$, that means,

$$
\xi_{*} T_{p} M \subset \mathcal{D}_{\xi(p)} \quad \text { for all } \quad p \in M .
$$

In particular, if $S$ is an m-dimensional affine submanifold of $N$, then the image $\xi^{S}(S)$ of the Gauß map $\xi^{S}$ is an integral manifold of $\mathcal{D}$.

From this proposition we derive the following local version of a general theorem on the reduction of codimension including the theorem of J. ERBACHER on the reduction of the codimension of isometric immersions in real space forms (see $[E]$ and $[R]$ p. 87) and several further results for specific ambient spaces $N$ (c.g. complex space forms and symmetric spaces).

Proposition 20 (Reduction of the codimension). If $\xi$ is a parallel strip in $N$ along a map $f: M \rightarrow N, S$ an affine submanifold of $N$ and if there exist points $p_{0} \in M$ and $q_{0} \in S$ such that $f\left(p_{0}\right)=q_{0}$ and $\xi\left(p_{0}\right)=T_{q_{0}} S$, then there exists a neighborhood $U$ of $p_{0}$ in $M$ such that $f \mid U$ is a $\mathrm{C}^{\infty}$-map into $S$ and $\xi(p)=T_{f(p)} S$ holds for all $p \in U$.

This is a special, but important case of a Theorem of P. Dombrowski (see [D], Section 3.4), for which we can give a short proof now.

Proof. From Proposition 19 and the integration theory of differentiable distributions (e.g. see [Nu, Theorem 4(iii)]) we obtain that $U:=\{p \in M \mid \xi(p) \in \hat{S}\}$ with $\hat{S}:=\xi^{S}(S)$ is an open subset of $M$ containing $p_{0}$ and that $\xi \mid U$ is a differentiable map into $\hat{S}$. Applying $\tau$ to this situation we get the desired assertion.

We will now show, how one can deduce an analogous result for strips with a parallel second fundamental form. In the rest of this section $N$ is a riemannian manifold.

Theorem 11 (Strips which osculate II-parallel submanifolds). If $\xi$ is a strip in $N$ along a map $f: M \rightarrow N$ with a $\nabla^{\mathbb{B}}$-parallel second fundamental form $h, S$ an II-parallel submanifold of $N$ and if there exist points $p_{0} \in M$ and $q_{0} \in S$ such that

$$
f\left(p_{0}\right)=q_{0}, \quad \xi\left(p_{0}\right)=T_{q_{0}} S \quad \text { and } \quad h\left(p_{0}\right)=h_{q_{0}}^{S},
$$

then there exists a neighborhood $U$ of $p_{0}$ in $M$ such that $f \mid U$ is a $\mathrm{C}^{\infty}$-map into $S$ and $\xi(p)=T_{f(p)} S$ and $h(p)=h_{f(p)}^{S}$ hold for all $p \in U$; loosely speaking, the strip $\xi \mid U$ osculates the submanifold $S$ along $f \mid U$ in second order.

This result can already be found in [R, Theorem 1]. But we will give now a very different proof demonstrating the efficiency of the methods developed in this thesis. 
Proof. According to Proposition 18(b) and Corollary $10 \hat{\xi}: p \rightarrow \mathcal{T}_{h(p)}$ is a parallel strip in $\hat{N}$ along $\xi$ and $\hat{S}:=\xi^{S}(S)$ an affine submanifold of $\hat{N}$. Because of the initial data we get from Example 10

$$
W_{0}:=\xi\left(p_{0}\right)=T_{q_{0}} S \in \hat{S} \quad \text { and } \quad \hat{\xi}\left(p_{0}\right)=\mathcal{T}_{h\left(p_{0}\right)}=\mathcal{T}_{h_{q_{0}}^{S}}=\xi_{*}^{S} T_{q_{0}} S=T_{W_{0}} \hat{S}
$$

Therefore, Proposition 20 guaranties that there exists a neighborhood $U$ of $p_{0}$ in $M$ such that $\xi \mid U$ is a $\mathrm{C}^{\infty}$-map into $\hat{S}$ and $\hat{\xi}(p)=T_{\xi(p)} \hat{S}$ holds for all $p \in U$. Applying $\tau$ resp. $\tau_{*}$ to this situation we get that $f \mid U$ is a $\mathrm{C}^{\infty}$-map into $S$ and

$$
\xi(p)=\tau_{*} \mathcal{T}_{h(p)}=\tau_{*} \hat{\xi}(p)=\tau_{*} T_{\xi(p)} \hat{S}=T_{f(p)} S .
$$

Furthermore, because of $\hat{\xi}(p)=\mathcal{T}_{h(p)}$ and $T_{\xi(p)} \hat{S}=\xi_{*}^{S} T_{f(p)} S=\mathcal{T}_{h_{f(p)}^{S}}$ the equality $\hat{\xi}(p)=T_{\xi(p)} \hat{S}$ also proves $h(p)=h_{f(p)}^{S}$ for all $p \in U$.

Example 13. Let $(c, \xi, h)$ be the prolongation of a triple $(p, W, b)$ in the direction $u \in W$ (in the sense of Definition 7) defined over an interval $J$. From Proposition 17 we know $\dot{\xi}(t) \in \mathcal{T}_{h(t)}$ for all $t$ and that $t \mapsto \mathcal{T}_{h(t)}$ is a parallel subbundle of $T \hat{N}$ along $\xi$. Therefore Proposition 18 shows that $\xi$ is a strip along $c$ with $\nabla^{\mathbb{B}}$-parallel second fundamental form $h$. In particular we see that each element $b \in \mathbb{B}$ occurs as second fundamental form of some strip. Now, let $S$ be some II-parallel submanifold of $N$ with $p \in S, W=T_{p} S$ and $b=h_{p}^{S}$. As $\xi(0)=W$ and $h(0)=b$ we can apply Theorem 11 and see that there exists some neighborhood $U$ of 0 in $J$ such that the strip $\xi \mid U$ osculates the submanifold $S$ along $c \mid U$ in second order. This result can also be derived from Example 11, which in addition shows that $c \mid U$ is a geodesic of $S$.

There are also global versions of Proposition 20 and Theorem 11 under suitable hypotheses on the submanifold $S$, which can be derived easily now.

\section{The generalization to indefinite metrics}

For simplicity I have supposed in the previous sections that $N$ is a riemannian manifold. In this section I will show that the main results, in particular those of the following list, remain valid in pseudoriemannian geometry:

- Theorem 1 on the II-parallelity of helical umbrellas,

- Theorem 6 on the second fundamental form of a Gauß map,

- Corollary 10 on the characterization of II-parallelity of a submanifold by its Gauß map,

- Theorem 8 on the construction of a II-parallel immersion $f: M \rightarrow N$ by projecting an affine immersions $\xi: M \rightarrow \hat{N}$,

- the globalization of Theorem 1 (see Theorem 9) and

- Theorem 11 on strips which osculate II-parallel submanifolds. 
First I will itemize the essential difficulties which may occur by handling an indefinite riemannian manifold $N:{ }^{18}$

(i) The indefinite inner product of $N$ does not induce a distance function. Therefore the notion of "completeness" makes no sense. Instead we must speak about geodesic completeness, which is characterized only by the fact that maximal geodesics are defined on the whole real line $\mathbb{R}$. In particular, the Theorem of Hopf-Rinow is not available.

(ii) We cannot concentrate on unit speed curves, because there also are curves in $N$ which may be lightlike at some points.

(iii) The Frenet apparatus is not available for arbitrary curves of $N$.

(iv) Also in the case of a geodesically complete space $N$ the maximal envelopment of a curve $C: J \rightarrow T_{q} N$ may only be defined on a proper "subinterval" of $J$ (see Definition 2).

(v) Pseudoriemannian submanifolds tangent to a given subspace $W \in \mathrm{G}_{m}\left(T_{q} N\right)$ can only exist if $W$ is non-degenerate, that means that the inner product of $T_{q} N$ is non-degenerate on $W$.

(vi) For a pseudoriemannian submanifolds $M$ and an arbitrary normal vector $z \in \perp M$ the shape operator $S_{z}^{M}$ may not be diagonalizable.

(vii) The orthogonal group $\mathrm{O}\left(T_{q} N\right)$ is not compact in the indefinite case and has four connected components.

The items (vi) and (vii) do not play any role for our consideration; the Theorem of Hopf-Rinow is not used.

In this section $N$ always denotes an $n$-dimensional connected pseudoriemannian manifold of index $k \in\{1, \ldots, n-1\} .{ }^{19}$ Let us now follow the preceding sections one after the other.

Ad Section 2. In order to avoid the difficulty (ii) we have already defined helical arcs of arbitrary velocity in Definition 3. Concerning (iii), the Frenet apparatus was only used in order to compare our definition of helical arcs with the common definition in the case of riemannian manifolds. A consequence of (iv) is that maximal helical arcs in a geodesical complete space $N$ may be defined only on some subsets $J \subsetneq \mathbb{R}$ in contrast to Remark 1(b); but from Proposition 3 we get:

Corollary 12. In a pseudoriemannian symmetric space $N$ every maximal helical arc is defined on $\mathbb{R}$.

Proof. Also for such symmetric space $N$ the Levi Civita connection coincides with its canonical connection as a homogeneous reductive space; thus $N$ is totally complete by Proposition 3 (b). Now the statement follows from Definition 3.

\footnotetext{
${ }^{18}$ We use the terminology of [ON2], where all basic facts of pseudoriemannian (= semiriemannian) geometry can be found.

${ }^{19}$ The case $k=n$ can be ignored, because it can be reduced to the riemannian case by replacing the metric $\langle\cdot, \cdot\rangle$ of $N$ by $-\langle\cdot, \cdot\rangle$.
} 
In this entire section $V$ a pseudoeuclidean vector space of index $k$, and $W \neq\{0\}$ a nondegenerate, proper subspace of $V$ (or $T_{p} N$, respectively) of index $\ell \in\{0, \ldots, \min \{m, k\}\}$. Since then $W^{\perp}$ is also non-degenerate and $V=W \oplus W^{\perp}$, we can define $A_{v}^{b}$ for arbitrary $(b, v) \in \mathrm{L}^{2}\left(W, W^{\perp}\right) \times W$, the tensor $R^{b}$ and the semiparallelity of $b$ for $b \in \mathrm{L}^{2}\left(W, W^{\perp}\right)$ as in Definition 1. Lemma 2 on the curvature invariance of subspaces $W$ and the semiparallelity of bilinear maps with respect to linear isometries remains valid without any change.

The parts (a) and (c) of Lemma 1 fail, because of analoguous reasons as for the above item (iii); but this Lemma was only important for Remark 1(a) and will not be used in the sequel.

Definition 2 and Proposition 2 are formulated for arbitrary "affine" manifolds $\left(N, \nabla^{N}\right)$ and are therefore also true for a pseudoriemannian $N$.

The Definition 4 of $M$-split-parallel displacement makes sense also in pseudoriemannian geometry for pseudoriemannian submanifolds $M$; in this situation Proposition 4 is also true. Furthermore, Proposition 5 on the geometry of II-parallel submanifolds in pseudoriemannian manifolds and Corollary 4 on the rigidity of submanifolds remains valid without any change. In spite of the previous item (iv), Proposition 8 and its proof yet remain valid for non-degenerate subspaces $W \subset T_{p} N$; thus the helical umbrella $M_{U}(p, W, b):=\exp ^{b}(U)$ associated with the triple $(p, W, b)$ exists (as pseudoriemannian submanifold of $N)$ for sufficiently small star shaped neighbourhoods $U$ of 0 in $W$.

Ad Section 3. The formulation of the pseudoriemannian version of Theorem 1 is now straight forward: We can define the linear isometries $\Phi_{u}^{b}$, the linear, non-degenerate subspaces $W_{u}$ (notice, $W$ is supposed to be non-degenerate) and the symmetric bilinear forms $b_{u} \in \mathrm{L}^{2}\left(W_{u}, W_{u}^{\perp}\right)$ as in the beginning of Section 3. Then Theorem 1, Remark 6, Corollary 5 on the II-parallelity of the helical umbrella $M_{U}(p, W, b)$ and Theorem 2 (now for pseudoriemannian symmetric spaces) remain valid. For the proof of the pseudoriemannian version of Theorem 1 see "Ad Section 8".

Ad Section 4. To my knowledge pseudoeuclidean Jordan triple systems were not yet discussed in literature; therefore I do not compare Ferus' characterization with the conditions (CI) and (SP) of Theorem 1 in the pseudoriemannian setting.

Ad Section 5. In the proof of Theorem 1, more exactly in the definition of the fibre bundle $\tau: \hat{N} \rightarrow N$, an essential modification is necessary, because we must restrict our consideration on $m$-dimensional submanifolds $M$ resp. subspaces $W$ of index $\ell$. For that we introduce for any $n$-dimensional pseudoeuclidean vector space $V$ of index $k$ the subset

$$
\mathrm{G}_{m}^{\ell}(V):=\left\{W \in \mathrm{G}_{m}(V) \mid W \text { is non-degenerate and of index } \ell\right\}
$$

of $\mathrm{G}_{m}(V)$. As before $\mathrm{O}(V)$ denotes the (pseudo-)orthogonal group of $V$ and End_ $(V)$ the subalgebra of skew-adjoint endomorphisms $V \rightarrow V$ now with respect to the indefinite inner product of $V$. Then $\mathrm{G}_{m}^{\ell}(V)$ is one of the orbits of the action $\psi: \mathrm{O}(V) \times \mathrm{G}_{m}(V) \rightarrow \mathrm{G}_{m}(V),(g, W) \mapsto$ $g(W)$, and already the neutral component of $\mathrm{O}(V)$ acts transitively on this orbit; hence $\mathrm{G}_{m}^{\ell}(V)$ bears the structure of a connected differentiable manifold. For a fixed element $W \in \mathrm{G}_{m}^{\ell}(V)$ the isotropy group of the action $\psi$ is

$$
\{A \in \mathrm{O}(V) \mid A(W)=W\} \cong \mathrm{O}(W) \times \mathrm{O}\left(W^{\perp}\right) .
$$


In fact, $\mathrm{G}_{m}^{\ell}(V)$ is a symmetric space isomorphic to $\mathrm{O}(V) / \mathrm{O}(W) \times \mathrm{O}\left(W^{\perp}\right)$; its symmetric structure is determined by the involution

$$
\mathrm{O}(V) \rightarrow \mathrm{O}(V), A \mapsto \sigma_{W} \circ A \circ \sigma_{W}^{-1}
$$

where $\sigma_{W} \in \mathrm{O}(V)$ denotes the reflection in the subspace $W$. Of course, $\mathrm{G}_{m}^{\ell}(V)$ is no symmetric subspace of $\mathrm{G}_{m}(V)$, but it is an open differentiable submanifold of $\mathrm{G}_{m}(V) .^{20}$

Proof of the previous statement. First notice that $\operatorname{dim} \mathrm{G}_{m}^{\ell}(V)=\operatorname{dim} \mathrm{G}_{m}(V)=m(n-m)$. Now, let us consider the following commutative diagram

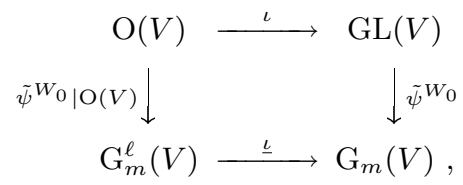

where $\iota$ and $\underline{\iota}$ denote the inclusion maps, $W_{0} \in \mathrm{G}_{m}^{\ell}(V)$ some subspace and $\tilde{\psi}^{W_{0}}$ the surjective submersion $g \mapsto g\left(W_{0}\right)$ induced by the canonical action $\tilde{\psi}: \mathrm{GL}(V) \times \mathrm{G}_{m}(V) \rightarrow \mathrm{G}_{m}(V)$. Also $\tilde{\psi}^{W_{0}} \mid \mathrm{O}(V): \mathrm{O}(V) \rightarrow \mathrm{G}_{m}^{\ell}(V)$ is a surjective submersion. As $\mathrm{O}(V)$ is a regular submanifold of $\mathrm{GL}(V)$, therefore $\_$is a differentiable map; moreover, it has constant rank, because it is equivariant with respect to the canonical actions of $\mathrm{O}(V)$ on $\mathrm{G}_{m}^{\ell}(V)$ resp. of $\mathrm{GL}(V)$ on $\mathrm{G}_{m}(V)$. Therefore, it suffices to show that $\underline{\iota}$ is immersive at the "point" $W_{0}$ (hence, because of $\operatorname{dim} \mathrm{G}_{m}^{\ell}(V)=\mathrm{G}_{m}(V)$ the map $\underline{\iota}$ is a diffeomorphism from $\mathrm{G}_{m}^{\ell}(V)$ onto an open subset of $\mathrm{G}_{m}(V)$, which is the desired result). In order to finish the proof we deduce from (149) the commutative diagram

$$
\begin{array}{crr}
T_{\mathrm{id}} \mathrm{O}(V) \stackrel{T_{\mathrm{id} \iota}}{\longrightarrow} & T_{\mathrm{id}} \mathrm{GL}(V) \\
T_{\mathrm{id}} \tilde{\psi}^{W_{0}} \mid \mathrm{O}(V) \downarrow & \downarrow T_{\mathrm{id}} \tilde{\psi}^{W_{0}} \\
T_{W_{0}} \mathrm{G}_{m}^{\ell}(V) \stackrel{T_{W_{0}} \stackrel{\iota}{\longrightarrow}}{\longrightarrow} T_{W_{0}} \mathrm{G}_{m}(V),
\end{array}
$$

and represent $T_{\mathrm{id}} \mathrm{O}(V)$ as $\mathfrak{h} \oplus \mathfrak{m}$ according to the symmetric space representation $\mathrm{G}_{m}^{\ell}(V) \cong \mathrm{O}(V) / \mathrm{O}\left(W_{0}\right) \times$ $\mathrm{O}\left(W_{0}^{\perp}\right)$; we have $\mathfrak{m} \cong\left\{A \in\right.$ End $_{-}(V) \mid A\left(W_{0}\right) \subset W_{0}^{\perp}$ and $\left.A\left(W_{0}^{\perp}\right) \subset W_{0}\right\} \subset T_{\text {id }} \mathrm{GL}(V)$. If now $v \in T_{W_{0}} \mathrm{G}_{m}^{\ell}(V)$ is a vector with $T_{W_{0}} \underline{\iota}(v)=0$, then there exists an element $A \in \mathfrak{m}$ with $T_{\text {id }}\left(\tilde{\psi}^{W_{0}} \mid \mathrm{O}(V)\right)(A)=v$; because of $(150)$ we have $T_{\mathrm{id}} \tilde{\psi}^{W_{0}}(A)=T_{W_{0}} \underline{\iota}(v)=0$. Therefore, $A$ is tangential to the isotropy group $\left\{B \in \operatorname{GL}(V) \mid B\left(W_{0}\right)=\right.$ $\left.W_{0}\right\}$ of $\tilde{\psi}$; therefrom we get $A\left(W_{0}\right) \subset W_{0}$. Because of $A \in \mathfrak{m}$ we deduce $A=0$ and hence $v=0$.

By $\mathbb{R}_{k}^{n}$ we denote the vector space $\mathbb{R}^{n}$ with the inner product

$$
\langle x, y\rangle=-\sum_{i=1}^{k} x_{i} y_{i}+\sum_{i=k+1}^{n} x_{i} y_{i}
$$

of index $k$. Now we specialize the previous consideration to $V=\mathbb{R}_{k}^{n}$, choose some subspace $V_{0} \in \mathrm{G}_{m}^{\ell}\left(\mathbb{R}_{k}^{n}\right)$, and introduce the subgroup $H \subset \mathrm{O}\left(\mathbb{R}_{k}^{n}\right)$, the subspace $\mathfrak{m} \subset$ End $_{-}\left(\mathbb{R}_{k}^{n}\right)$ and the isomorphism $\theta: \mathfrak{m} \rightarrow T_{V_{0}} \mathrm{G}_{m}^{\ell}\left(\mathbb{R}_{k}^{n}\right)$ as in the formulas (53), (57) and (59) (replacing always $\mathbb{R}^{n}$ by $\left.\mathbb{R}_{k}^{n}\right)$.

In contrast to Section 5 now $\tau: \hat{N} \rightarrow N$ denotes the Graßmann bundle over $N$ whose fibre $\hat{N}_{q}$ over $q \in N$ is $\mathrm{G}_{m}^{\ell}\left(T_{q} N\right)$. Its differentiable structure is given by the fact that this bundle is associated with the bundle $\mathbb{O}(N)$ of orthonormal frames (the structure group of which is $\left.\mathrm{O}\left(\mathbb{R}_{k}^{n}\right)\right)$ via the map

$$
\hat{\rho}: \mathbb{O}(N) \times \mathrm{G}_{m}^{\ell}\left(\mathbb{R}_{k}^{n}\right) \rightarrow \hat{N},(u, W) \mapsto u(W)
$$

\footnotetext{
${ }^{20}$ One can show that the set $\mathrm{G}_{m}^{*}(V)$ of all non-degenerate subspaces $W \in \mathrm{G}_{m}(V)$ is an open, dense subset of $\mathrm{G}_{m}(V)$ and that $\mathrm{G}_{m}^{\ell}(V)$ is a connected component of $\mathrm{G}_{m}^{*}(V)$, see [Pa], p. 26.
} 
Remark 20. In order to compare this Graßmann bundle with that from Section 5 we denote the previous bundle by $\tau^{(k, \ell)}: \hat{N}^{(k, \ell)} \rightarrow N$ in this remark and the bundle from Section 5 by $\tau^{(0,0)}: \hat{N}^{(0,0)} \rightarrow N$. As $\tau^{(0,0)}$ is associated with the linear frame bundle $\mathrm{L}(N)$ (see $(85)$ ) and the principal bundle $\mathbb{O}(N)$ is a reduction of $\mathrm{L}(N)$ (see $[\mathrm{KN}]$ Vol.1, p. 53), the bundle $\tau^{(0,0)}$ is also associated with $\mathbb{O}(N)$, namely via the map

$$
\mathbb{O}(N) \times \mathrm{G}_{m}\left(\mathbb{R}^{n}\right) \rightarrow \hat{N}^{(0,0)},(u, W) \mapsto u(W) .
$$

Since $\mathrm{G}_{m}^{\ell}\left(\mathbb{R}_{k}^{n}\right)$ is an open submanifold of $\mathrm{G}_{m}\left(\mathbb{R}^{n}\right)$, therefore $\tau^{(k, \ell)}$ is an associated subbundle of $\tau^{(0,0)}$ and $\hat{N}^{(k, \ell)}$ an open differentiable submanifold of $\hat{N}^{(0,0)}$.

Now we can follow the constructions of Section 5 without any changes: We introduce the pull back bundle $\mathbb{E}:=\tau^{*} T N=\hat{N} \times{ }_{N} T N$. Since every $W \in \mathrm{G}_{m}^{\ell}\left(T_{q} N\right)$ is non-degenerate, we get the orthogonal splitting $\mathbb{E}_{W}=W \oplus W^{\perp}$ exactly as in the riemannian case; this induces the orthogonal vector bundle splitting $\mathbb{E}=\pi \oplus \Perp$. On this basis we can define the principal fibre bundle $\pi^{\mathbb{P}}: \mathbb{P}=\mathbb{O}^{\top} \times \hat{N} \mathbb{O}^{\perp} \rightarrow \hat{N}$ of the adapted orthonormal frames of $\mathbb{E}$ (whose structure group is the isotropy group $H$ ) and the connection $\mathcal{H}^{\mathbb{P}}$. Furthermore, the construction of the associated vector bundles End $_{-}(\mathbb{E}), \mathbb{H}, \mathbb{M}$ and $\mathbb{B}$ together with their induced linear connections is immediate. Moreover, we have the isomorphisms $\Theta_{W}: \mathbb{M}_{W} \rightarrow T_{W} \mathrm{G}_{m}^{\ell}\left(T_{q} N\right)$ for all $W \in \hat{N}_{q}$ (because $\mathrm{G}_{m}^{\ell}\left(T_{q} N\right)$ is a symmetric space) and the universal second fundamental form $\hat{\nu}: T \hat{N} \rightarrow \mathbb{M}$. All results on these data described in Section 5 are true without any change in the present situation. In particular, Example 9 is applicable for any $m$-dimensional pseudoriemannian submanifold $M \subset N$ of index $\ell$; notice that its Gauß map $\xi^{M}: p \mapsto$ $T_{p} M$ takes its values in the present bundle space $\hat{N}$. Furthermore, Lemma 5 on the parallel displacement in $\mathbb{E}$ remains valid; and Remark 10 on $\mathbb{P}$-morphisms is of general nature and therefore it is also true in the present situation.

Ad Section 6. In order to define the linear connection $\hat{\nabla}$ on the present manifold $\hat{N}$ we have to make slight modifications in Section 6. As the group $\mathrm{GL}\left(\mathbb{R}^{n}\right)$ does not act on the "restricted" Graßmann manifold $\mathrm{G}_{m}^{\ell}\left(\mathbb{R}_{k}^{n}\right)$ we must replace it by the group $\mathrm{O}\left(\mathbb{R}_{k}^{n}\right)$; consequently we can not use the situation of (85), but we must consider the present Graßmann bundle $\tau: \hat{N} \rightarrow N$ as being associated with the principal fibre bundle $\mathbb{O}(N)$ of orthonormal frames of $T N$ via the map $\hat{\rho}: \mathbb{O}(N) \times \mathrm{G}_{m}^{\ell}\left(\mathbb{R}_{k}^{n}\right) \rightarrow \hat{N}$ described in (151). As in Section 6 the linear connection of $\mathbb{O}(N)$ corresponding to $\nabla^{N}$ induces a connection $\hat{\mathcal{H}}$ on the bundle $\tau: \hat{N} \rightarrow N ; \hat{\mathcal{H}}$-horizontal curves in $\hat{N}$ are again characterized by formula (86). ${ }^{21}$ Furthermore, we introduce the projection $\nu: T \hat{N} \rightarrow \hat{\mathcal{V}}$ corresponding to the splitting $T \hat{N}=\hat{\mathcal{H}} \oplus \hat{\mathcal{V}}$. Then Theorem 4 remains valid; it means, the isomorphisms $\Theta_{W}$ are the "fibres" of a vector bundle $\mathbb{P}$-isomorphism $\Theta: \mathbb{M} \rightarrow \hat{\mathcal{V}}$, the identity $\Theta \circ \hat{\nu}=\nu$ holds and

$$
(\hat{\eta}, \hat{\nu}): T \hat{N} \rightarrow \mathbb{E} \oplus \mathbb{M} \quad \text { with } \quad \hat{\eta}: T \hat{N} \rightarrow \mathbb{E}, \hat{v} \mapsto \tau_{*} \hat{v}
$$

is a fibre bundle isomorphism. The proof for this theorem must be adapted to the present situation by using that $\hat{\mathcal{V}}$ is a fibre bundle over $N$ associated with $\mathbb{O}(N)$, namely via the map $\rho^{\hat{\mathcal{v}}}: \mathbb{O}(N) \times T \mathrm{G}_{m}^{\ell}\left(\mathbb{R}_{k}^{n}\right) \rightarrow \hat{\mathcal{V}},(u, w) \mapsto \hat{\rho}_{u *} w$ with $\hat{\rho}_{u}:=\hat{\rho}(u, \cdot): \mathrm{G}_{m}^{\ell}\left(\mathbb{R}_{k}^{n}\right) \rightarrow \hat{N}$ (notice (151)).

\footnotetext{
${ }^{21}$ Of course, in Section 6 it would be possible also to associate the bundle $\tau$ with the principal bundle $\mathbb{O}(N)$ as was described in Remark 20; but there we prefered the association with the full frame bundle $\mathrm{L}(N)$, since we liked to refer to this general situation when we introduced the Tsukada distribution $\mathcal{D}$ for an affine manifold $\left(N, \nabla^{N}\right)$ in Section 10 before Proposition 19.
} 
As the bundle space $\mathbb{P}$ coincides with the bundle space $\mathbb{O}(N)$ and the projection of the latter bundle is exactly $\tau \circ \pi^{\mathbb{P}}$, we find that $\hat{\mathcal{V}}$ is a vector bundle over $\hat{N}$ associated with the principal bundle $\mathbb{P}$ via the restriction $\rho^{\hat{\nu}} \mid\left(\mathbb{P} \times T_{V_{0}} \mathrm{G}_{m}^{\ell}\left(\mathbb{R}_{k}^{n}\right)\right)$. Replacing the map $\theta$ from the proof of Theorem 4 by the $H$-equivariant isomorphism $\theta: \mathfrak{m} \rightarrow T_{V_{0}} \mathrm{G}_{m}^{\ell}\left(\mathbb{R}_{k}^{n}\right)$ from above we can now follow the proof of Theorem 4 .

Because of Theorem 4 the tangent bundle $T \hat{N}$ can be considered as being associated with $\mathbb{P}$ (with typical fibre $\mathbb{R}^{n} \oplus \mathfrak{m}$ ) via the map $\rho^{T \hat{N}}$ described in (89) and (90). Therefore, also in the present situation the manifold $\hat{N}$ bears a linear connection $\hat{\nabla}$ which comes from the connection $\mathcal{H}^{\mathbb{P}}$. It is characterized by the statement (91). Proposition 10 describing the properties of $\hat{\nabla}$ and Proposition 10 characterizing the geodesics of $(\hat{N}, \hat{\nabla})$ remain valid. But instead of Corollary 7 we have only the following version:

Corollary 13. For every pseudoriemannian symmetric space $N$ the associated space $\hat{N}$ is geodesically complete.

Proof. According to Corollary 12 all maximal helical arcs are defined on $\mathbb{R}$. Now use Proposition 11.

Now we can follow Section 6 to show that Theorem 6 is true for the second fundamental form of the Gauß map $\xi^{M}$ of every pseudoriemannian submanifold $M \subset N$ of index $\ell$, and that Corollary 10 characterizes as well the II-parallelity of an isometric immersion $f: M \rightarrow N$ from a pseudoriemannian manifold $M$ of index $\ell$.

Ad Section 7. Without any change we can define the distinguished subspaces $\mathcal{T}_{b} \subset T_{W} \hat{N}$ for all $b \in \mathbb{B}(W \in \hat{N})$ such that Proposition 13, Proposition 14 on the invariance properties of these subspaces and Proposition 15 on the parallelity of the map $b \mapsto \mathcal{T}_{b}$ hold. Furthermore, for each pseudoriemannian submanifold $M \subset N$ of index $\ell$ its Gauß map satisfies $\xi_{*}^{M} T_{p} M=\mathcal{T}_{h_{p}^{M}}$ for every $p \in M$ (see Example 10), and the Remark 15 on the van der Waerden-Bortolotti connection and the general Remark 16 on the $\mathbb{P}$-morphism $\mathcal{T}: \mathbb{B} \rightarrow \hat{\hat{N}}$ remain valid. However, Theorem 8 on the construction of a II-parallel immersion $f: M \rightarrow N$ by projecting an affine immersions $\xi: M \rightarrow \hat{N}$ requires an additional statement, namely that $f^{*}\langle\cdot, \cdot\rangle_{N}$ really is a pseudoriemannian metric of index $\ell$; this follows easily from the fact that $\xi$ is the Gauß map of $f$; apart from that one can follow the proof of Theorem 8. Finally, the prolongation of a triple $(q, W, b)$ (with $W \in \mathrm{G}_{m}^{\ell}\left(T_{q} N\right)$ ) in direction of a vector $u \in W$ can be defined as in Definition 7; then Remark 18 and Proposition 17 are true without any change.

Ad Section 8. Now, we can follow the proof of Theorem 1; the following ingredients, justified above, (and no others) are used: Proposition 8, Lemma 5, Proposition 13, Proposition 14, Theorem 8, Definition 7 and Proposition 17. Hence, Theorem 1 holds also in the pseudoriemannian situation.

Ad Section 9. Concerning the pseudoriemannian globalization of Theorem 1 it is clear that the subspaces $W$ in the triples $(q, W, b) \in \mathcal{B}_{N}$ are now supposed to be non-degenerate and of index $\ell$ and that Definition 9(b) of geodesically closed II-parallel isometric immersions can be 
generalized to the pseudoriemannian case in an obvious way. But notice that for such an immersion the geodesical completeness of $M$ can only be deduced in the case of a pseudoriemannian symmetric space $N$ (by means of Corollary 12); insofar Remark 19 is only valid partially.

The main assertion of Theorem 9 remains valid, if we replace the terms "riemannian" and "complete" by "pseudoriemannian" and "geodesically complete". However the completeness assertion is only valid (as geodesical completeness) for pseudoriemannian symmetric ambient spaces $N$. With the same modifications also Theorem 10 on the geodesical completion of IIparallel submanifolds of pseudoriemannian symmetric spaces remains true. It should be noticed that the affine version of Lemma 9 was cited at the end of Section 9 in order to have no difficulties in handling the pseudoriemannian situation here.

Ad Section 10. In the situation, where the ambient manifold $N$ is pseudoriemannian, it is natural to restrict the consideration to such strips $\xi: M \rightarrow T N$ along maps $f: M \rightarrow N$, for which the subspaces $\xi(p) \subset T_{f}(p)$ are non-degenerate; if $M$ is connected, then the index of these subspaces is the same for all $p \in M$; in order to integrat the consideration into the framework of the present Graßmann bundle $\tau: \hat{N} \rightarrow N$, we should suppose always that this index is $\ell$. With these modifications in mind the whole content of Section 10 (in particular, Theorem 11 on strips which oscualte II-parallel submanifolds) remains valid in the pseudoriemannian theory.

\section{II-parallel isometric immersions adapted to special riemannian geometries}

In this section we will suppose that the manifold $N$ is eqipped with a additional geometric structure and we will ask for the existence of II-parallel submanifolds of $N$, which in some sense are compatible with this structure. For simplicity we assume again that the riemannian metric of $N$ is positive definite.

Let $V$ be a euclidean vector space, $K$ a closed subgroup of $\mathrm{O}(V)$ and $m$ an integer with $1 \leq m<\operatorname{dim} V$.

Then certain linear subspaces $W \in \mathrm{G}_{m}(V)$ are distinguished:

Definition 11. We say that a subspace $W \in \mathrm{G}_{m}(V)$ is adapted to the subgroup $K$, if the reflection $\sigma_{W}: V \rightarrow V$ in the subspace $W$ satisfies

$$
\sigma_{W} \circ K \circ \sigma_{W}^{-1}=K
$$

Proposition 21. Let $W_{0} \in \mathrm{G}_{m}(V)$ be a subspace, which is adapted to $K$.

(a) Every element of the orbit

$$
K W_{0}=\left\{g\left(W_{0}\right) \in \mathrm{G}_{m}(V) \mid g \in K\right\}
$$

is also adapted to $K$.

(b) If $K$ is connected, then $K W_{0}$ is a symmetric subspace of the riemannian symmetric space $\mathrm{G}_{m}(V)$ and therefore a compact totally geodesic submanifold of $\mathrm{G}_{m}(V)$. 
Proof. For (a). For $W:=g\left(W_{0}\right)$ with $g \in K$ one has $\sigma_{W}=g \circ \sigma_{W_{0}} \circ g^{-1}$. This implies the assertion.

For (b). $\Sigma: \mathrm{O}(V) \rightarrow \mathrm{O}(V), g \mapsto \sigma_{W_{0}} \circ g \circ \sigma_{W_{0}}^{-1}$ is the involution defining the symmetric space structure of $\mathrm{G}_{m}(V)$ with respect to the reference point $W_{0}$. Because of (152) (applied to $\left.W=W_{0}\right)$ therefore $\Sigma \mid K$ is an involution of the subgroup $K$ endowing the orbit $K W_{0}$ with the structure of a symmetric subspace.

In the examples of this section each of the subgroups $K \subset \mathrm{O}(V)$ is constructed by means of a tensor $j$ of some type $(k, r)$ on $V$, namely by

$$
K(j):=\{g \in \mathrm{O}(V) \mid g \cdot j=j\},
$$

where $\mathrm{O}(V)$ acts on the space $\mathcal{T}^{k, r}(V)$ of tensors of type $(k, r)$ canonically from the left; ${ }^{22}$ obviously $K(j)$ is the isotropy group of this action for the element $j$.

Lemma 10. Suppose that $j$ is symmetric resp. antisymmetric with respect to a subspace $W \in \mathrm{G}_{m}(V)$, i.e,

$$
\sigma_{W} \cdot j=j \quad \text { resp. } \quad \sigma_{W} \cdot j=-j .
$$

Then $W$ is adapted to the subgroup $K(j)$ and also to its neutral connected component $K_{0}(j)$.

The elementary proof is left to the reader.

Example 14. (a) Suppose that $V$ even is a unitary space with complex structure $J \in$ End_ $(V)$ :

$$
J^{2}=-\mathrm{id}_{V} \quad \text { and } \quad \forall x, y \in V:\langle J x, J y\rangle=\langle x, y\rangle .
$$

Then $K(J)$ is the unitary group $\mathrm{U}(V)$, and $J$ is symmetric resp. antisymmetric with respect to a subspace $W \in \mathrm{G}_{m}(V)$ if and only if $W$ is a complex resp. a Lagrangian subspace; the latter means $J W=W^{\perp}$, what only is possible for $m=n$. Notice, that also in this situation $\mathrm{G}_{m}(V)$ denotes the real Graßmann manifold. By means of Lemma 10 the complex and the Lagrangian subspaces are adapted to the subgroup $U(V)$.

(b) Let $N$ be a simply connected riemannian symmetric space, $p \in N, V:=T_{p} N$ and $j$ the curvature tensor $R_{p}^{N}$ of $N$ at $p$. The tensor $R_{p}^{N}$ is symmetric with respect to a subspace $W \in \mathrm{G}_{m}\left(T_{p} N\right)$ (in the sense of the preceding Lemma) if and only if $W$ is strongly curvature invariant, i.e., both $W$ and $W^{\perp}$ are $R_{p}^{N}$-invariant (the implication " $\Rightarrow$ " is trivial, and for the implication " $\Leftarrow$ " one uses the symmetry properties of $R_{p}^{N}$ ). By means of Lemma 10 the strongly curvature invariant subspaces of $T_{p} N$ are adapted to the subgroup $K\left(R_{p}^{N}\right)$ and also to its neutral component $K_{0}\left(R_{p}^{N}\right)$ (see (156)). - Now let $I(N)$ denote the isometry group of $N$ and $I(N)_{p}$ its isotropy group at the point $p$. Since we obiously have

$$
K\left(R_{p}^{N}\right)=\left\{g \in \mathrm{O}\left(T_{p} N\right) \mid \forall u, v, w \in T_{p} N: g\left(R_{p}^{N}(u, v) w\right)=R_{p}^{N}(g u, g v) g w\right\},
$$

the Theorem of Cartan-Ambrose-Hicks implies that the map

$$
I(N)_{p} \rightarrow K\left(R_{p}^{N}\right), f \mapsto T_{p} f
$$

\footnotetext{
${ }^{22} \mathrm{~A}$ special case was already used in the preparations of Theorem 2 , where we considered the action $(g, R) \mapsto$ $g_{\mathfrak{C}} R$ of $\mathrm{O}(V)$ on the space $\mathfrak{C}(V)$ of curvature like tensors.
} 
is a Lie group isomorphism. Furthermore, if $G$ denotes the neutral connected component of $I(N)$ and $G_{p} \subset G$ its isotropy group at $p$, then obviously the neutral component $\left(I(N)_{p}\right)_{0}$ of $I(N)_{p}$ is contained in $G$ and therefore we have $\left(I(N)_{p}\right)_{0} \subset G_{p} \subset I(N)_{p}$. Thus the induced map $G /\left(I(N)_{p}\right)_{0} \rightarrow G / G_{p}$ is a connected covering of the simply connected space $G / G_{p} \cong N$. Consequently we get

$$
\left(I(N)_{p}\right)_{0}=G_{p}
$$

and therefore

$$
K_{0}\left(R_{p}^{N}\right)=\left\{T_{p} f \mid f \in G_{p}\right\} .
$$

Remark. After the above discussion we see that for every subspace $W \in \mathrm{G}_{m}\left(T_{p} N\right)$ the following assertions (i) $-(\mathrm{v})$ are pairwise equivalent:

(i) $W$ is strongly curvature invariant.

(ii) $W^{\perp}$ is strongly curvature invariant.

(iii) $R_{p}^{N}$ is symmetric with respect to $W^{\perp}$.

(iv) The reflection $\sigma_{W^{\perp}} \in \mathrm{O}\left(T_{p} N\right.$ in the subspace $W^{\perp}$ is contained in the subgroup $K\left(R_{p}^{N}\right)$.

(v) There exists an isometry $\bar{\sigma}_{W} \in I(N)_{p}$ with $T_{p} \bar{\sigma}_{p}=\sigma_{W^{\perp}}$.

The equivalence (i) $\Leftrightarrow(\mathrm{v})$ in the previous remark is of interest in the theory of extrinsic symmetric submanifolds, as one can see from the following definition, which can be found at the beginning of [Na1].

Definition 12. Let $N$ be a simply connected riemannian symmetric space. A quasiregular ${ }^{23}$ submanifold $M \subset N$ is said to be extrinsic symmetric, if for every point $q \in M$ there exists an isometry $\bar{\sigma}_{q} \in I(N)_{q}$ such that $T_{q} \bar{\sigma}_{q} \in \mathrm{O}\left(T_{q} N\right)$ is the reflection $\sigma_{\perp_{q} M}$ in the normal space $\perp_{q} M$ and $\bar{\sigma}_{q}(M)=M$. (Then $M$ also is a symmetric space, namely with the geodesic symmetries $\sigma_{q}=\bar{\sigma}_{q} \mid M$.) Thus one defines more generally: An isometric immersion $f: M \rightarrow N$ is said to be extrinsic symmetric, if

(i) $M$ is a riemannian symmetric space with the geodesic symmetries $\sigma_{q}(q \in M)$,

(ii) for each point $q \in M$ there exists an isometry $\bar{\sigma}_{q} \in I(N)_{f(q)}$ such that $T_{f(q)} \bar{\sigma}_{q} \in$ $\mathrm{O}\left(T_{f(q)} N\right)$ is the reflection $\sigma_{\perp_{q} f}$ in the normal space $\perp_{q} f:=\left(f_{*} T_{q} M\right)^{\perp}$, and

(iii) the isometries $\sigma_{q}$ and $\bar{\sigma}_{q}$ from (i) and (ii) satisfy $f \circ \sigma_{q}=\bar{\sigma}_{q} \circ f$.

Proposition 22. If $N$ is a simply connected riemannian symmetric space, then the following assertions are true:

(a) Every extrinsic symmetric immersion $f: M \rightarrow N$ is II-parallel.

(b) If $f: M \rightarrow N$ is a II-parallel isometric immersion from a connected riemannian symmetric space $M$ (see Corollary 2 and Theorem 10), then $f$ is extrinsic symmetric if and only if the images $f_{*} T_{q} M(q \in M)$ are strongly curvature invariant ${ }^{24}$.

\footnotetext{
${ }^{23}$ Notice, a submanifold $M \subset N$ is said to be quasiregular, iff every differentiable map $f: L \rightarrow N$ with $f(L) \subset M$ is also differentiable as a map into $M$.

${ }^{24}$ Because of Proposition 5(a) this condition is already satisfied, if all normal spaces $\perp_{q} f:=\left(f_{*} T_{q} M\right)^{\perp}$ are curvature invariant.
} 
For the sake of completeness I will give a proof, although the proposition is well known (see [Na1], p. 218). - It is worth to be mentioned that Definition 12 can also be applied in the situation of an arbitrary riemannian manfiold $N$ and that then Proposition 22(a) remains valid.

Proof. For (a). Let be given an extrinsic symmetric immersion $f: M \rightarrow N$, a point $q \in M$ and vector fiedls $X, Y, Z \in \Gamma(T M)$, and let $\sigma:=\sigma_{q}$ and $\bar{\sigma}:=\bar{\sigma}_{q}$ denote the isometries from the items (i) and (ii) of Definition 12. Using item (iii) of this definition we deduce from $\bar{\sigma}_{*} \nabla_{Y}^{N} f_{*} Z=\nabla^{N} \bar{\sigma}_{*} f_{*} Z$ by means of the Gauß equation

$$
\bar{\sigma}_{*} h^{f}(Y, Z)=h^{f}\left(\sigma_{*} Y, \sigma_{*} Z\right) .
$$

Continuing analogously with the Weingarten equation we furthermore derive

$$
\bar{\sigma}_{*} \nabla_{X}^{\perp} h^{f}(Y, Z)=\nabla_{X}^{\perp} h^{f}\left(\sigma_{*} Y, \sigma_{*} Z\right) .
$$

Combining these two results we get

$$
\bar{\sigma}_{*}\left(\bar{\nabla} h^{f}\right)(X, Y, Z)=\left(\bar{\nabla} h^{f}\right)\left(\sigma_{*} X, \sigma_{*} Y, \sigma_{*} Z\right) .
$$

Evaluating this equation at the point $q$ we get $\left(\bar{\nabla}_{X_{q}} h^{f}\right)\left(Y_{q}, Z_{q}\right)=-\left(\bar{\nabla}_{X_{q}} h^{f}\right)\left(Y_{q}, Z_{q}\right)$, i.e. $\left(\bar{\nabla}_{X_{q}} h^{f}\right)\left(Y_{q}, Z_{q}\right)=0$. Hence, $f$ is II-parallel.

For (b): Let a II-parallel isometric immersion $f: M \rightarrow N$ be given and assume that $M$ is a riemannian symmetric space. Thus condition (i) of Definition 12 is satisfied for $f$ and condition (ii) is equivalent to the strong curvature invariance of the spaces $f_{*} T_{q} M(q \in M)$ by means of the remark in Example 14(b). Therefore, it suffices to deduce condition (iii) from (ii) using the II-parallelity of $f$. For that define the two II-parallel immersions $f_{1}:=f \circ \sigma_{q}$ and $f_{2}:=\bar{\sigma}_{q} \circ f$ for an arbitrary point $q \in M$; they have the same geometric 2-jet at $q: f_{1}(q)=f(q)=f_{2}(q)$, $T_{q} f_{1}=-T_{q} f=T_{q} f_{2}$ and $h_{q}^{f_{1}}=h_{q}^{f}=h_{q}^{f_{2}}$. By generalizing Corollary 4 to II-parallel immersions we find $f_{1}=f_{2}$, q.e.d.

Now, we will introduce the notion of $K$-structures on $N$. A good idea is to do this via a reduction of the linear frame bundle. Nevertheless, I will give another definition, which is more flexible for our purpose. In the following remark we will see how Definition 13 can be subordinated to the usual definition. - As before, $V$ denotes a euclidean vector space, but now we suppose $\operatorname{dim} V=\operatorname{dim} N$. And we will not work with a closed subgroup $K$ of $\mathrm{O}(V)$, but more generally with a faithful (= injective) representation $\psi: K \rightarrow \mathrm{O}(V)$ from a connected, compact Lie group $K$, the Lie algebra of which is denoted by $\mathfrak{k}$.

Definition 13. A $K$-structure on $N$ is defined to be a principal fibre bundle $\mathbb{K}$ with structure group $K$ such that $T N$ is an associated vector bundle with typical fibre $(V, \psi)$ via an appropriate association map

$$
\rho: \mathbb{K} \times V \rightarrow T N
$$

the maps $\rho_{u}: V \rightarrow T_{p} N\left(u \in \mathbb{K}_{p}, p \in N\right)$ of which are linear isometries; furthermore, the Levi Civita connection on $T N$ shall be induced by some $K$-invariant connection $\mathcal{H}^{\mathbb{K}}$ on $\mathbb{K}$.

Notice, in this case the characteristic equation of association maps says

$$
\forall(u, g, x) \in \mathbb{K} \times K \times V: \quad \rho(u g, x)=\rho(u, \psi(g) x) .
$$


Of course, the Graßmann bundle $\tau: \hat{N} \rightarrow N$, too, is associated with $\mathbb{K}$, namely via the map

$$
\hat{\rho}: \mathbb{K} \times \mathrm{G}_{m}(V) \rightarrow \hat{N},(u, W) \mapsto \rho_{u}(W),
$$

(notice that $K$ acts on $\mathrm{G}_{m}(V)$ canonically via $\psi$ ) and the connection $\hat{\mathcal{H}}$ on the bundle $\tau$ (described in the beginning of Section 6) is then also deduced by $\mathcal{H}^{\mathbb{K}}$, namely by $\hat{\mathcal{H}}_{\hat{\rho}(u, W)}=$ $\hat{\rho}_{*}^{W} \mathcal{H}_{u}^{\mathbb{K}}$.

Remark 21. If $\mathbb{K}$ is a reduction of the orthonormal frame bundle $\mathbb{O}(N)$ to a connected, closed subgroup $K \subset \mathrm{O}\left(\mathbb{R}^{n}\right)$ and if the canonical connection of $\mathbb{O}(N)$ induces a connection $\tilde{\mathcal{H}}$ on $\mathbb{K}$ (by restriction), then we obtain a $K$-structure on $N$ in the sense of Definition 13 by the following choices: $V:=\mathbb{R}^{n}$ and $\rho: \mathbb{K} \times V \rightarrow T N,(u, x) \mapsto u(x)$. - Conversely, in the situation of Definition 13 choose a linear isometry $\Phi: \mathbb{R}^{n} \rightarrow V$. Then $P:=\left\{\rho_{u} \circ \Phi \in \mathbb{O}(N) \mid u \in \mathbb{K}\right\}$ is a reduction of the orthonormal frame bundle $\mathbb{O}(N)$ to the closed subgroup $\Phi^{-1} \circ K \circ \Phi \subset \mathrm{O}\left(\mathbb{R}^{n}\right)$, and if we pull back the connection of $\mathbb{K}$ via $\Phi$ onto $P$, then the latter is the restriction of the canonical connection of $\mathbb{O}(N)$.

Example 15. (a) Let $N$ be a Kähler manifold of complex dimension $n$ (notice that here we diverge from our usual meaning of $n)$. Then the hermitian frame bundle $\mathbb{U}(N)$ defines a $\mathrm{U}(n)$-structure according to the recipe of Remark 21 . Notice, that because of the parallelity of the complex structure of $N$ the Levi Civita connection of $N$ is induced by a $U(n)$ invariant connection of $\mathbb{U}(N)$.

(b) In the situation of Example 14(b) let $\phi: G \times N \rightarrow N$ with $G:=I_{0}(N)$ denote the canonical action, fix a reference point $p \in N$, choose $V=T_{p} N$ and $K:=G_{p}$ and let $\psi: G_{p} \rightarrow \mathrm{O}\left(T_{p} N\right)$ be its isotropy representation $f \mapsto T_{p} f$; notice that $G_{p}$ is connected because of (155). Then the principal fibre bundle $\phi^{p}: G \rightarrow N$ together with the association map $\rho: G \times T_{p} N \rightarrow T N,(f, v) \mapsto f_{*} v$ is a $G_{p}$-structure on $N$. By formula (14) in the proof of Proposition 3 there was described a $G_{p}$-invariant connection of the fibre bundle $\phi^{p}: G \rightarrow N$, which induces the canonical connection on the symmetric space $N$; this connection coincides with the Levi Civita connection. Furthermore, notice that we have $\psi\left(G_{p}\right)=\left\{T_{p} f \mid f \in G_{p}\right\}=K_{0}\left(R_{p}^{N}\right)$ by means of $(156)$.

From now on we suppose the situation of Definition 13.

The following simple construction of new bundles associated with $\mathbb{K}$ will become of importance for us. Let $\mathrm{FB}(E) \rightarrow N$ be a fibre bundle with typical fibre $E$ which is associated with $\mathbb{K}$ by a map $\rho^{E}: \mathbb{K} \times E \rightarrow \mathrm{FB}(E)$; saying this we incorporate that the group $K$ acts on $E$ from the left. If $E^{\prime}$ is a $K$-invariant submanifold of $E$, then $\operatorname{FB}\left(E^{\prime}\right):=\rho^{E}\left(\mathbb{K} \times E^{\prime}\right)$ is a submanifold of $\mathrm{FB}(E)$ and with the canonically induced projection $\mathrm{FB}\left(E^{\prime}\right) \rightarrow N$ we obtain a subbundle of $\mathrm{FB}(E) \rightarrow N$, which is also associated with $\mathbb{K}$. With respect to the connection $\mathcal{H}^{\mathrm{FB}(E)}$ of $\mathrm{FB}(E) \rightarrow N$ induced by $\mathcal{H}^{\mathbb{K}}$ the subbundle $\mathrm{FB}\left(E^{\prime}\right) \rightarrow N$ is parallel, i.e., $\mathrm{FB}\left(E^{\prime}\right)$ is invariant with respect to the parallel transport in $\mathrm{FB}(E)$ along arbitrary curves $\alpha: J \rightarrow N$ :

$$
\left(\|_{t_{0}}^{t_{1}} \alpha\right)^{\mathrm{FB}(E)}\left(\operatorname{FB}\left(E^{\prime}\right)_{\alpha\left(t_{0}\right)}\right)=\operatorname{FB}\left(E^{\prime}\right)_{\alpha\left(t_{1}\right)} \quad \text { for all } \quad t_{0}, t_{1} \in J
$$

in other words: The connection $\mathcal{H}^{\mathrm{FB}\left(E^{\prime}\right)}$ induced by $\mathcal{H}^{\mathbb{K}}$ on the subbundle $\operatorname{FB}\left(E^{\prime}\right)$ is given by

$$
\mathcal{H}_{a}^{\mathrm{FB}\left(E^{\prime}\right)}=\mathcal{H}_{a}^{\mathrm{FB}(E)} \text { for all } \quad a \in \mathrm{FB}\left(E^{\prime}\right)
$$


Example 16. (a) The Lie group bundle $\mathrm{O}(T N) \rightarrow N$ (with fibres $\mathrm{O}(T N)_{q}=\mathrm{O}\left(T_{q} N\right)$ ) is canonically associated with $\mathbb{K}$. It is subordinated to the preceding consideration by $E:=$ $\mathrm{O}(V)$, on which $K$ acts by $(k, g) \mapsto \psi(k) \circ g \circ \psi(k)^{-1}$, and by $\rho^{E}(u, g)=\rho_{u} \circ g \circ \rho_{u}^{-1}$. Furthermore, $K^{\prime}:=\psi(K)$ is a $K$-invariant Lie subgroup of $\mathrm{O}(V)$. Thus, we obtain a parallel Lie group subbundle $\mathrm{FB}\left(K^{\prime}\right) \rightarrow N$ of the bundle $\mathrm{O}(T N) \rightarrow N$. We abbreviate

$$
K_{q}:=\operatorname{FB}\left(K^{\prime}\right)_{q} \quad \text { and have } K_{q}=\rho_{u} \circ K^{\prime} \circ \rho_{u}^{-1} \quad \text { with } \quad u \in \mathbb{K}_{q} .
$$

(b) The Lie algebra bundle End $(T N) \rightarrow N$ (with fibres End $(T N)_{q}=$ End $_{-}\left(T_{q} N\right)$ ) is canonically associated with $\mathbb{K}$. It is subordinated to the preceding consideration by $E:=$ End $_{-}(V)$, on which $K$ acts by $(g, A) \mapsto \psi(g) \circ A \circ \psi(g)^{-1}$, and by $\rho^{E}(u, A)=\rho_{u} \circ A \circ \rho_{u}^{-1}$. Furthermore, the Lie algebra $\mathfrak{k}^{\prime} \subset$ End_ $(V)$ of $K^{\prime}=\psi(K)$ is a $K$-invariant Lie subalgebra of End_ $(V)$. Thus, we obtain a parallel Lie algebra subbundle $\mathrm{FB}\left(\mathfrak{k}^{\prime}\right) \rightarrow N$ of the bundle End_ $_{-}(T N) \rightarrow N$, therefore being also parallel in the vector bundle $\operatorname{End}(T N) \rightarrow N$. We abbreviate

$$
\mathfrak{k}_{q}:=\mathrm{FB}\left(\mathfrak{k}^{\prime}\right)_{q} \quad \text { and have } \mathfrak{k}_{q}=\rho_{u} \circ \mathfrak{k}^{\prime} \circ \rho_{u}^{-1} \text { with } u \in \mathbb{K}_{q} .
$$

Obviously $\mathfrak{k}_{q}$ is the Lie algebra of the Lie group $K_{q}$ introduced in (a). ${ }^{25}$

(c) As already mentioned above the Graßmann bundle $\tau: \hat{N}=\mathrm{G}_{m}(T N) \rightarrow N$ is associated with $\mathbb{K}$. Let us fix some subspace $W_{0} \in \mathrm{G}_{m}(V)$ which is adapted to $K^{\prime}=\psi(K)$. Obviously, the orbit $K^{\prime} W_{0}$ is a $K$-invariant submanifold of the typical fibre $\mathrm{G}_{m}(V)$ of $\tau$. Thus, we obtain a parallel subbundle

$$
\hat{N}\left(W_{0}\right):=\operatorname{FB}\left(K^{\prime} W_{0}\right) \rightarrow N
$$

of the bundle $\tau: \hat{N} \rightarrow N$. Let us fix a point $q \in N$ and an element $u \in \mathbb{K}_{q}$ and put $W_{u}:=\rho_{u}\left(W_{0}\right)$. As $\rho_{u}: V \rightarrow T_{q} N$ is an isometry, we derive from the situation in the typical fibre $V$ :

(i) The fibre $\hat{N}\left(W_{0}\right)_{q}:=\mathrm{FB}\left(K^{\prime} W_{0}\right)_{q}$ is a symmetric subspace of the symmetric space $\hat{N}_{q}=\mathrm{G}_{m}\left(T_{q} N\right)$; see Proposition 21(b).

(ii) $K_{q}=\rho_{u} \circ K^{\prime} \circ \rho_{u}^{-1}$ is a closed subgroup of $\mathrm{O}\left(T_{q} N\right)$ (see (a)).

(iii) The subspace $W_{u} \in \hat{N}\left(W_{0}\right)_{q}$ is adapted to the subgroup $K_{q}$.

(iv) The fibre $\hat{N}\left(W_{0}\right)_{q}$ coincides with the orbit $K_{q} W_{u}$.

Furthermore, we have

(v) $\hat{N}\left(W_{0}\right)=\left\{\rho_{u} \circ \psi(g)\left(W_{0}\right) \mid u \in \mathbb{K}, g \in K\right\}$.

Moreover, Proposition 21(a) and the previous statements show that every subspace $W \in$ $\hat{N}\left(W_{0}\right)_{q}$ also is adapted to $K_{q}$. If we repeat the proof of Proposition 21(b) in the situation (iv), we find that the Cartan decomposition of $\mathfrak{k}_{q}$ corresponding to the symmetric space $\hat{N}\left(W_{0}\right)_{q}$ at a reference point $W \in \hat{N}\left(W_{0}\right)_{q}$ is

$$
\mathfrak{k}_{q}=\left(\mathfrak{k}_{q} \cap \mathbb{H}_{W}\right) \oplus\left(\mathfrak{k}_{q} \cap \mathbb{M}_{W}\right) \quad(\text { see }(\mathrm{b})) \quad ;
$$

\footnotetext{
${ }^{25}$ Let $\mathfrak{g}^{\mathbb{K}}$ denote the Lie algebra bundle over $N$ whose fibre $\mathfrak{g}_{q}^{\mathbb{K}}$ is the Lie algebra of vector fields $X \in \Gamma\left(T \mathbb{K}_{q}\right)$ satisfying $\mathrm{R}_{g_{*}} X=X \circ \mathrm{R}_{g}$ for all $g \in K$. This bundle is canonically associated to $\mathbb{K}$; its typical fibre is (k, Ad). Since the faithful representation $\psi: V \rightarrow \mathrm{O}(V)$ delivers a $K$-equivariant Lie algebra isomorphism $\mathfrak{k} \rightarrow \mathfrak{k}^{\prime}$, this isomorphism furthermore induces a Lie algebra bundle isomorphism $\mathfrak{g}^{\mathbb{K}} \rightarrow \mathrm{FB}\left(\mathfrak{k}^{\prime}\right)$.
} 
for the meaning of $\mathbb{H}_{W}$ and $\mathbb{M}_{W}$ see (54) and (55). From this equation we immediately obtain that the tangent spaces of the submanifold $\hat{N}\left(W_{0}\right)_{q} \subset \hat{N}_{q}$ are given by

$$
T_{W}\left(\hat{N}\left(W_{0}\right)_{q}\right)=\Theta_{W}\left(\mathfrak{k}_{q} \cap \mathbb{M}_{W}\right) \text { for all } W \in \hat{N}\left(W_{0}\right)_{q},
$$

with the isomorphism $\Theta_{W}: \mathbb{M}_{W} \rightarrow T_{W} \mathrm{G}_{m}\left(T_{q} N\right)$ defined in (60). Let us also repeat the statement (158) in the present situation:

$$
\mathcal{H}_{W}^{\hat{N}\left(W_{0}\right)}=\hat{\mathcal{H}}_{W} \quad \text { for all } \quad W \in \hat{N}\left(W_{0}\right) .
$$

Finally notice that the submanifold $\hat{N}\left(W_{0}\right)$ is closed and regular in $N$, as can be seen easily by use of local trivializations of the bundle $\tau$ and the compactness of the orbit $K^{\prime} W_{0}$.

Proposition 23. At every "point" $W \in \hat{N}\left(W_{0}\right)_{q}$ the tangent space of the entire submanifold $\hat{N}\left(W_{0}\right) \subset \hat{N}$ is given by

$$
T_{W} \hat{N}\left(W_{0}\right)=\left\{\hat{v} \in T_{W} \hat{N} \mid \hat{\nu}(\hat{v}) \in \mathfrak{k}_{q}\right\}
$$

Proof. The connection of the fibre bundle $\hat{N}\left(W_{0}\right) \rightarrow N$ gives the splitting

$$
T_{W} \hat{N}\left(W_{0}\right)=T_{W}\left(\hat{N}\left(W_{0}\right)_{q}\right) \oplus \mathcal{H}_{W}^{\hat{N}\left(W_{0}\right)} \underset{(161),(162)}{=} \Theta_{W}\left(\mathfrak{k}_{q} \cap \mathbb{M}_{W}\right) \oplus \hat{\mathcal{H}}_{W}
$$

Now we split an arbitrary vector $\hat{v} \in T_{W} \hat{N}$ according to the splitting $T_{W} \hat{N}=\hat{\mathcal{V}}_{W} \oplus \hat{\mathcal{H}}_{W}$, i.e.: $\hat{v}=\nu(\hat{v})+\eta(v)$ (see the beginning of Section 6). Because of (163) we see: $\hat{v} \in T_{W} \hat{N}\left(W_{0}\right)$ if and only if $\Theta_{W} \circ \hat{\nu}(\hat{v})=\nu(\hat{v}) \in \Theta_{W}\left(\mathfrak{k}_{q} \cap \mathbb{M}_{W}\right)$; here we have made use of Theorem 4. Therefrom we derive the assertion immediately.

Proposition 24. $\hat{N}\left(W_{0}\right)$ is an affine (=autoparallel) submanifold of $\hat{N}$.

Proof. Let $\hat{X}, \hat{Y} \in \Gamma\left(T \hat{N}\left(W_{0}\right)\right)$ and $Z \in \Gamma(\mathbb{E})$ be given. Notice that $Z$ also can be considered as a vector field of $N$ along $\tau$. Then we calculate:

$$
\begin{aligned}
\left(\hat{\nu}\left(\hat{\nabla}_{\hat{X}} \hat{Y}\right)\right) Z & \underset{(92)}{=}\left(\nabla_{\hat{X}}^{\mathbb{M}} \hat{\nu}(\hat{Y})\right) Z \underset{(70)}{=} \nabla_{\hat{X}}^{\mathbb{E}}(\hat{\nu}(\hat{Y}) Z)-\hat{\nu}(\hat{Y})\left(\nabla_{\hat{X}}^{\mathbb{E}} Z\right) \\
& =\nabla_{\hat{X}}^{N}(\hat{\nu}(\hat{Y}) Z)-\hat{\nu}(\hat{X}) \hat{\nu}(\hat{Y}) Z-\hat{\nu}(\hat{Y})\left(\nabla^{N}{ }_{\hat{X}} Z-\hat{\nu}(\hat{X}) Z\right) \\
& =\left(\nabla_{\hat{X}}^{\operatorname{End}(T N)} \hat{\nu}(\hat{Y})-[\hat{\nu}(\hat{X}), \hat{\nu}(\hat{Y})]\right) Z .
\end{aligned}
$$

As $\hat{\nu}(\hat{Y})$ is a section in the vector bundle $\operatorname{FB}\left(\mathfrak{k}^{\prime}\right)$ because of Proposition 23, we obtain from Example 16(b) $\nabla^{\operatorname{End}(T N)} \hat{X} \hat{\nu}(\hat{Y})=\nabla^{\mathrm{FB}\left(\mathfrak{k}^{\prime}\right)} \hat{X} \hat{\nu}(\hat{Y}) \in \Gamma\left(\mathrm{FB}\left(\mathfrak{k}^{\prime}\right)\right)$; moreover, we also have $[\hat{\nu}(\hat{X}), \hat{\nu}(\hat{Y})] \in \Gamma\left(\operatorname{FB}\left(\mathfrak{k}^{\prime}\right)\right)$, as $\mathrm{FB}\left(\mathfrak{k}^{\prime}\right)$ is a Lie algebra bundle. Thus we have derived $\hat{\nu}\left(\hat{\nabla}_{\hat{X}} \hat{Y}\right)=$ $\nabla^{\mathrm{FB}\left(\mathfrak{k}^{\prime}\right)} \hat{X} \hat{\nu}(\hat{Y})-[\hat{\nu}(\hat{X}), \hat{\nu}(\hat{Y})] \in \Gamma\left(\mathrm{FB}\left(\mathfrak{k}^{\prime}\right)\right)$. Applying Proposition 23 again we therefore obtain $\hat{\nabla}_{\hat{X}} \hat{Y} \in \Gamma\left(T \hat{N}\left(W_{0}\right)\right)$. This characterizes $\hat{N}\left(W_{0}\right)$ as being an affine submanifold.

Theorem 12. If $f: M \rightarrow N$ is an II-parallel isometric immersion from an m-dimensional connected riemannian manifold $M$ and if for some point $p \in M$ its Gauß map $\xi:=\xi^{f}: M \rightarrow$ $\hat{N}, p \mapsto f_{*} T_{p} M$ satisfies $W:=\xi^{f}(p) \in \hat{N}\left(W_{0}\right)$ and $\xi_{*}^{f} T_{p} M \subset T_{W} \hat{N}\left(W_{0}\right)$, then $\xi^{f}$ is a $\mathrm{C}^{\infty}$ map into $\hat{N}\left(W_{0}\right)$. 
Proof. As stated in Example 16(c) the submanifold $\hat{N}\left(W_{0}\right)$ of $\hat{N}$ is closed and regular. Therefore, bearing Proposition 24 in mind we can apply the two parts of Lemma 8 to the affine immersion $\xi^{f}$ (see Corollary 10).

We will give now two applications of the previous proposition.

First, we assume that $N$ is a Kählerian manifold with complex structure $J$. Then a submanifold $M \subset N$ is said to be complex resp. Lagrangian iff for every $p \in M$ the tangent space $T_{p} M$ is a complex resp. Lagrangian subspace of the hermitian vector space $T_{p} N$. By means of the Gauß and Weingarten equation one deduces immediately: If $M$ is a complex resp. Lagrangian submanifold of $N$, then the second fundamental form $h^{M}$ resp. the shape operator $S^{M}$ of $M$ satisfy

$$
J h^{M}(X, Y)=h^{M}(X, J Y) \quad \text { resp. } J h^{M}(X, Y)=-S_{J Y}^{M} X
$$

for all vector fields $X, Y \in \Gamma(T M)$.

Corollary 14. For every connected II-parallel submanifold $M$ of a Kählerian manifold $N$ (with complex structure $J$ ) the following assertions are true:

(a) If at some point $p \in M$ the tangent space $T_{p} M$ is a complex subspace of $T_{p} N$ and we have

$$
\forall u, v \in T_{p} M: \quad J h^{M}(u, v)=h^{M}(u, J v),
$$

then $M$ is a complex submanifold of $N$.

(b) If at some point $p \in M$ the tangent space $T_{p} M$ is a Lagrangian subspace of $T_{p} N$ and we have

$$
\forall u, v \in T_{p} M: \quad J h^{M}(u, v)=-S_{J v}^{M} u,
$$

then $M$ is a Lagrangian submanifold of $N$.

Proof. We use the notations of the Examples 15(a) and the results of Example 14(a). Furthermore we use that for every hermitian frame $u$ of $N$ at $p$ we have $\rho_{u}=u$. Therefore, we obtain from Example 16(a) and (b): $K_{p}=u \circ \mathrm{U}(n) \circ u^{-1}=\mathrm{U}\left(T_{p} N\right)=\left\{g \in \mathrm{O}\left(T_{p} N\right) \mid g \circ J=J \circ g\right\}$, hence $\mathfrak{k}_{p}=\left\{A \in \operatorname{End}_{-}\left(T_{p} N\right) \mid A \circ J=J \circ A\right\}$. Let us abbreviate $W:=T_{p} M=\xi^{M}(p)$ and $b:=h_{p}^{M}$.

For (a). Suppose that the complex dimension of $T_{p} M$ is $m$. The subspace $W_{0}:=\mathbb{C}^{m} \times\{0\}$ of the typical fibre $V=\mathbb{C}^{n}$ is adapted to the subgroup $U(n) \subset \mathrm{O}(V)$. One sees immediately that $\hat{N}\left(W_{0}\right)_{q}$ is the subset of all complex linear subspaces $\subset T_{q} N$ of complex dimension $m$ for every $q \in N$. Therefore, $M$ is a complex submanifold of $N$, if $\xi^{M}(M) \subset \hat{N}\left(W_{0}\right)$ holds. From the hypothesis we know $W \in \hat{N}\left(W_{0}\right)$. Thus, according to Theorem 12 it is sufficient to deduce $\xi_{*}^{M} T_{p} M \subset T_{W} \hat{N}\left(W_{0}\right)$ from Equation (164). Now this equation implies also $\forall z \in$ $W^{\perp}: S_{J z}=J S_{z}$. Because of Example 2(a) we therefore get: $\forall u \in W: A_{u}^{b} \circ J=J \circ A_{u}^{b}$, that means $\hat{\nu}\left(\xi_{*}^{M} u\right)=A_{u}^{b} \in \mathfrak{k}_{p}$; for the preceding equation we have used (79). Thus we obtain $\xi_{*}^{M} T_{p} M \subset T_{W} \hat{N}\left(W_{0}\right)$ by means of Proposition 23 .

For (b). Suppose $\operatorname{dim} T_{p} M=m$. The subspace $W_{0}:=\mathbb{R}^{n}$ of the typical fibre $V=\mathbb{C}^{n}$ is adapted to the subgroup $U(n) \subset \mathrm{O}(V)$. As $\mathrm{U}(n) W_{0}$ is the subset of all Lagrangian subspaces of $\mathbb{C}^{n}$, we find that $\hat{N}\left(W_{0}\right)_{q}$ is the subset of all Lagrangian subspaces $\subset T_{q} N$ for every $q \in$ $N$. Therefore, $M$ is a Lagrangian submanifold of $N$, if $\xi^{M}(M) \subset \hat{N}\left(W_{0}\right)$ holds. From the 
hypothesis we know $W \in \hat{N}\left(W_{0}\right)$. Thus, according to Theorem 12 it is sufficient to deduce $\xi_{*}^{M} T_{p} M \subset T_{W} \hat{N}\left(W_{0}\right)$ from Equation (165). Because of Example 2(a) this equation can be formulated as $\forall u, v \in W: J A_{u}^{b} v=A_{u}^{b} J v$. Substituting $z:=J v \in \perp_{p} M$ in this equation, we find $\hat{\nu}\left(\xi_{*}^{M} u\right)=A_{u}^{b} \in \mathfrak{k}_{p}$ as in the proof of (a); thus we complete the argumentation.

For the second application of Theorem 12 we make some preparation:

Lemma 11. Let $N$ be a complete riemannian manifold, $G:=I_{0}(N)$ the neutral component of its isometry group and $G_{p}$ its isotropy group at a point $p \in N$. Then the Lie algebra of $G$ resp. of $G_{p}$ can be identified with the Lie algebra $\mathfrak{g}$ of the Killing vector fields on $N$ (see $[K N], p$. 239) resp. with its subalgebra $\mathfrak{g}_{p}=\left\{X \in \mathfrak{g} \mid X_{p}=0\right\}$. Moreover, the linearization $\psi_{L}: \mathfrak{g}_{p} \rightarrow$ End $_{-}\left(T_{p} N\right)$ of the isotropy representation $\psi: G_{p} \rightarrow \mathrm{O}\left(T_{p} N\right), f \mapsto T_{p} f$ is given by

$$
\psi_{L}(X)=\nabla^{N} X \mid T_{p} N: v \mapsto \nabla_{v}^{N} X .
$$

Proof. For $\mathfrak{g}_{p}=\left\{X \in \mathfrak{g} \mid X_{p}=0\right\}:$ Let $X \in \mathfrak{g}$ be given and let $\Phi^{X}$ denote its flow. Then we have the following sequence of equivalences: $X \in \mathfrak{g}_{p} \Leftrightarrow \forall t \in \mathbb{R}: \Phi^{X}(t, p)=p \Leftrightarrow X_{p}=0$.

For $\psi_{L}$ : Let $X \in \mathfrak{g}_{p}$ and $\Phi^{X}$ be as before, fix some $v \in T_{p} N$ and choose a vector field $Y \in \Gamma(T N)$ with $Y_{p}=v$. Using the definition of $\psi$ and $\Phi^{X}(t, p) \equiv p$ we get

$$
\psi\left(\Phi_{t}^{X}\right) v=\left(T_{p} \Phi_{t}^{X}\right) Y_{p}=\Phi_{-t *}^{-X} Y \circ \Phi_{t}^{-X}(p),
$$

thus

$$
\psi_{L}(X) v=\left.\frac{d}{d t}\right|_{t=0}\left(\psi\left(\Phi_{t}^{X}\right) v\right)=[-X, Y]_{p}=\nabla^{N}{ }_{v} X-\nabla^{N} X_{p}=0 Y=\nabla^{N}{ }_{v} X .
$$

Now, we assume that $N$ is a simply connected riemannian symmetric space. we fix some point $p \in N$ and a strongly curvature invariant subspace $W_{0} \subset \mathrm{G}_{m}\left(T_{p} N\right)$ and use the notations of the Examples 14(b), 15(b) and 16(a) - (c). We get:

(i) $W_{0}$ is adapted to $K^{\prime}=\psi\left(G_{p}\right)=\left\{T_{p} f \mid f \in G_{p}\right\}$ (see Example 14(b) and 15(b)),

(ii) $\mathbb{K}_{p}=G_{p}$ (see the definition of $\mathbb{K}$ in Example $15(\mathrm{~b})$ ),

(iii) $K_{p}=K^{\prime}$ (see formula (159) and the definition of $\rho$ in Example 15(b)),

(iv) $\mathfrak{k}_{p}=\left\{\nabla^{N} X\left|T_{p} N\right| X \in \Gamma(T N)\right.$ a Killing field with $\left.X_{p}=0\right\}$

$=\left\{A \in\right.$ End $\left._{-}\left(T_{p} N\right) \mid A_{\mathfrak{C}} R_{p}^{N}=0\right\}$ (see Example 16(b), (i), (iii), Lemma 11 and (156)), remember that $A_{\mathfrak{C}}$ denotes the action of $A$ as a derivative on the vector space $\mathfrak{C}\left(T_{p} N\right)$ of algebraic curvature tensors; see formula (39).

(v) $\hat{N}\left(W_{0}\right)=G W_{0}:=\left\{\left(T_{p} f\right)\left(W_{0}\right) \mid f \in G\right\}$ with $G:=I_{0}(N)$ (see assertion (v) in Example 16(c).

Now we introduce two subsets $\mathcal{B}_{\text {esym }}(N)$ and $\mathcal{B}_{\text {esym }}^{0}(N)$ of the set $\mathcal{B}_{N}$ defined in Definition 8 , which will become of importance immediately:

$$
\mathcal{B}_{\text {esym }}(N):=\left\{(p, W, b) \in \mathcal{B}_{N} \mid W \in \hat{N}_{p} \text { strongly curvature invariant and } \forall u \in W: A_{u}^{b} \in \mathfrak{k}_{p}\right\}
$$


and

$$
\mathcal{B}_{\text {esym }}^{0}(N):=\left\{(p, W, b) \in \mathcal{B}_{\text {esym }}(N) \mid b \text { is } R_{p}^{N} \text {-semiparallel }\right\}
$$

Example 17. If $N$ is a real space form, then every subspace $W \subset T_{p} N$ is strongly curvature invariant and we have $A_{\mathfrak{C}} R_{p}^{N}=0$ for every element $A \in \operatorname{End}_{-}\left(T_{p} N\right)$. Therefore, we get $\mathcal{B}_{\text {esym }}(N)=\mathcal{B}_{N}$ and $\mathcal{B}_{\text {esym }}^{0}(N)=\left\{(p, W, b) \in \mathcal{B}_{N} \mid b\right.$ is $R_{p}^{N}$-semiparallel $\}$.

Corollary 15. For every II-parallel isometric immersion $f: M \rightarrow N$ from a connected riemannian symmetric space $M$ (see Corollary 2) into a simply connected riemannian symmetric space $N$ the following three assertions are pairwise equivalent:

(a) $M$ is an extrinsic symmetric submanifold of $N$.

(b) For every point $p \in M$ the triple $\left(f(p), f_{*} T_{p} M, \hat{h}_{p}^{f}\right)$ belongs to the set $\mathcal{B}_{\text {esym }}^{0}(N)$.

(c) There exists a point $p \in M$ such that the triple $\left(f(p), f_{*} T_{p} M, \hat{h}_{p}^{f}\right)$ belongs to the set $\mathcal{B}_{\text {esym }}(N)$.

Remember, $\hat{h}^{f}$ is the section in $\mathbb{B}$ along the Gauß map $\xi^{f}: p \mapsto f_{*} T_{p} M$ characterized by

$$
\hat{h}_{p}^{f}\left(f_{*} v, f_{*} w\right)=h_{p}^{f}(v, w) \quad \text { for all } \quad v, w \in T_{p} M(p \in M) \quad(\text { see Remark 13(c)). }
$$

Proof. Obviously it suffices to prove the implications (a) $\Rightarrow$ (b) and (c) $\Rightarrow$ (a).

For $(\mathrm{a}) \Rightarrow(\mathrm{b})$. Fix some point $p \in M$ and put $W_{0}:=f_{*} T_{p} M$ and $b:=\hat{h}_{p}^{f}$. If (a) is satisfied, we know from Proposition 22(b) that $W_{0}$ is strongly curvature invariant; hence we can work with the notations and the assertions, which are formulated above in the items (i) - (v). In particular, $\hat{N}\left(W_{0}\right)$ is the orbit $G W_{0} \cdot{ }^{26}$ We will now show

$$
\xi^{f}(M) \subset \hat{N}\left(W_{0}\right)
$$

For that let $\tilde{p} \in M$ be another point, choose a geodesic $c: \mathbb{R} \rightarrow M$ with $c(0)=p$ and $c(2)=\tilde{p}$, put $q:=c(1)$ and let $\sigma_{q}$ and $\bar{\sigma}_{q}$ be the isometries described in Definition 12. Then we have $\sigma_{q}(p)=\tilde{p}$ and therefore also $\sigma_{q *} T_{p} M=T_{\tilde{p}} M$. Consequently we get:

$$
\xi^{f}(\tilde{p})=f_{*} T_{\tilde{p}} M=\left(f \circ \sigma_{q}\right)_{*} T_{p} M=\left(\bar{\sigma}_{q} \circ f\right)_{*} T_{p} M=\bar{\sigma}_{q *}\left(W_{0}\right)=\left(\bar{\sigma}_{q} \circ \bar{\sigma}_{p}\right)_{*}\left(W_{0}\right) .
$$

As $\gamma: t \mapsto \bar{\sigma}_{c(t)} \circ \bar{\sigma}_{p}$ is a continuous curve in $I(N)$ with $\gamma(0)=\operatorname{id}_{N}$ (even more, on page 219 of [Na1] is shown that $\gamma$ is a differentiable 1-parameter subgroup of $I(N))$, we find $\gamma(\mathbb{R}) \subset G$. In particular we have $\gamma(1) \in G$, hence $\xi^{f}(\tilde{p})=\left(T_{p} \gamma(1)\right)\left(W_{0}\right) \in G W_{0}=\hat{N}\left(W_{0}\right)$; thus (167) is proved. As $\hat{N}\left(W_{0}\right)$ is a regular submanifold of $\hat{N},(167)$ implies that $\xi^{f}$ is a differentiable map into $\hat{N}\left(W_{0}\right)$. Hence we get $\xi_{*}^{f} T_{p} M \subset T_{W_{0}} \hat{N}\left(W_{0}\right)$. Therefore, Proposition 23 and Formula (79) yield $A_{u}^{b}=\hat{\nu}\left(\xi_{*}^{f} u\right) \in \mathfrak{k}_{p}$ for every $u \in T_{p} M$. Thus we have obtained $\left(f(p), f_{*} T_{p} M, \hat{h}_{p}^{f}\right) \in \mathcal{B}_{\text {esym }}(N)$. Eventually, the II-parallelity of $f$ implies the semiparallelity of $b$ (see Proposition 5(b)).

For (c) $\Rightarrow\left(\right.$ a). Assume the situation of (c). Then $W_{0}:=f_{*} T_{p} M$ is strongly curvature invariant; and $b:=\hat{h}_{p}^{f}$ satisfies $\forall u \in W_{0}: \hat{\nu}\left(\xi_{*}^{f} u\right)=A_{u}^{b} \in \mathfrak{k}_{p}$. By means of Proposition 23 we get

\footnotetext{
${ }^{26}$ Some authors denote such an orbit by $\mathcal{O}$ and say that a submanifold $M \subset N$ is a $\mathcal{O}$-submanifold, if $T_{p} M \in \mathcal{O}$ for every $p \in M$.
} 
$\xi_{*}^{f} T_{p} M \subset T_{W_{0}} \hat{N}\left(W_{0}\right)$. Therefore we can apply Theorem 12 and find that $\xi^{f}$ is a map into $\hat{N}\left(W_{0}\right)$, i.e.: $\forall q \in M: f_{*} T_{q} M \in \hat{N}\left(W_{0}\right)$. As all subspaces $W \in \hat{N}\left(W_{0}\right)=G W_{0}$ are strongly curvature invariant, Proposition 22 implies assertion (a).

The previous Example 17 shows that the following theorem is a strong generalization of the Addendum to Theorem 9 concerning II-parallel submanifolds in spaces of constant curvature (see also Corollary 5). Notice, that II-parallel isometric immersions from a symmetric space into a real space form automatically are extrinsic symmetric (see $[\mathrm{F}, \mathrm{Ta}, \mathrm{BR}]$ ).

Theorem 13. If $N$ is a simply connected riemannian symmetric space, $q$ a point in $N, W$ an $m$-dimensional subspace of $T_{q} N$ and $b: W \times W \rightarrow W^{\perp}$ a symmetric bilinear map, then there exists an extrinsic symmetric immersion $f: M \rightarrow N$ from a connected riemannian symmetric space $M$ such that $f(p)=q, f_{*} T_{p} M=W$ and $\hat{h}_{p}^{f}=b$ hold for some point $p \in M$ if and only if

(a) $W$ is strongly curvature invariant,

(b) $\quad\left(A_{u}^{b}\right)_{\mathfrak{C}} R_{q}^{N}=0$ for all $u \in W$, and

(c) $\quad b$ is $R_{q}^{N}$-semiparallel.

It should be mentioned that NAITOH has given another algebraic description of the initial data $(q, W, b)$ of extrinsic symmetric immersions in [Na1] and that the articles [NT, B-T] are based on his description.

Proof. First notice that the conditions (a) - (c) exactly mean $(q, W, b) \in \mathcal{B}_{\text {esym }}^{0}(N)$. Therefore, Prop 22(a) and the implication (a) $\Rightarrow$ (b) of Corollary 15 show that these conditions are necessary for the existence of the extrinsic symmetric immersion $f$. Now we prove that they are also sufficient: For that let $\tilde{M}=M_{U}(q, W, b)=\exp ^{b}(U)$ be the helical umbrella described in Proposition 8. For any fixed vector $u \in W$ the function $R_{u}(t)$ defined in Remark 6 is constant, namely $\forall t \in \mathbb{R}: R_{u}(t)=R_{q}^{N}$, because of condtion (b) and Equation (40); thus the conditions (CI') and (SP') of Remark 6 are satisfied because of (a) and (c), whence $\tilde{M}$ is a II-parallel submanifold.

Applying Theorem 10 we find that there exists a II-parallel isometric immersion $f: M \rightarrow N$ from a symmetric space $M$ such that $f(p)=q, f_{*} T_{p} M=W$ and $\hat{h}_{p}^{f}=b$ hold for some point $p \in M$. Eventually, the implication (c) $\Rightarrow$ (a) of Corollary 15 implies that $f$ is extrinsic symmetric. 


\section{References}

[A] W. Ambrose: Higher order Grassmann bundles, Topology 3 (1964) suppl.2, 199 238.

[BR] R.A. BACKes, H. RECKZIEGEL: On symmetric submanifolds of spaces of constant curvature, Math. Ann. 263 (1983) 419 - 433.

[B-T] J. Berndt, J.-H. Eschenburg, H. Naitoh, K. Tsukada: Symmetric submanifolds associated with irreducible symmetric R-spaces, Math. Ann. 332 (2005) 721 - 737.

[Be] A.L. Besse: Einstein manifolds, Springer 1987.

[BL] W. Blaschke, K. Leichtweiss : Elementare Differentialgeometrie, Springer 1985.

[BD] T. Bröcker, T. том Dieck: Representations of compact Lie groups, Springer 1973.

[By] W.M. Bоотнвy: An introduction to differential manifolds and riemannian geometry, Acad. Press 1986, 2nd ed.

[Bo] Bourbaki, N.: Variétés Différentielles et Analytiques, Fascicule de Résultats, Hermann 1967.

[C] Cartan, É.: Leçons sur la géométrie des espaces de Riemann, Gauthier-Villars 1946.

[Ca] Cartan, H.: Calcul différentiel, Hermann 1967.

[Ch] B.-Y. Chen: Geometry of submanifolds, Marcel Dekker 1973.

[CO] B.-Y. Chen, K. Ogiue: On totally real submanifolds, Trans. Amer. Math. Soc. 193 (1974) $257-266$.

[D] P. Dombrowski: Differentiable maps into riemannian manifolds of constant stable osculating rank, part 1, J. Reine Angew. Math. 274/275 (1975) 310 - 341.

[E] J. ERbacher: Reduction of the codimension of an isometric immersion, J. Diff. Geom. 5 (1971) $333-340$.

[F] D. Ferus: Symmetric submanifolds of Euclidean space, Math. Ann. 247 (1980) $81-$ 93.

[He] S. Helgason: Differential geometry, Lie groups, and symmetric spaces, Academic Press, 1978.

[Hi] N. Hicks: A theorem on affine connexions, Illinois J. Math. Ann. 3 (1959) $242-254$.

[JR] T. Jentsch, H. Reckziegel: Submanifolds with parallel second fundamental form studied via the Gauß map, Annals of Global Analysis and Geometry 29 (2006) 51 93.

[KN] S. Kobayashi, K. Nomizu: Foundations of Differential Geometry, Vol. $1 \& 2$, Interscience Publ. 1963/1969.

[LN] D.S. Leung, K. Nomizu: The axiom of spheres in Riemannian geometry, J. Diff. Geometry 5 (1971) $487-489$. 
[L] Ü. Lumiste: Submanifolds with parallel fundamental form. F. Dillen (ed.) et al., Handbook of differential geometry. Vol. I. Amsterdam: North-Holland (2000) $779-864$

[Na1] H. NAITOH: Symmetric submanifolds of compact symmetric spaces, Tsukuba J. Math. 10 (1986) $215-242$

[Na2] H. NaITOH: Symmetric submanifolds and generlized Gauss map, Tsukuba J. Math. $14(1990) 113-132$

[NT] H. Naitoh, M. TAkeuchi: Symmetric submanifolds of symmetric spaces, Sugaku 2 (1989) $157-188$

[NY] K. Nomizu, K. Yano: On circles and spheres in Riemannian geometry, Math. Ann. 210 (1974) $163-170$.

[Nu] F. NüBEL: On integral manifolds for vector space distributions. Math. Ann. 294 (1992) $1-7$.

[ON1] B. O'NeILl: The fundamental equations of a submersion. Mich. Math. J. 13 (1966) $459-469$.

[ON2] B. O’Neill: Semi-riemannian geometry, Academic Press 1983.

[P] W.A. Poor: Differential Geometric Structures, McGraw-Hill 1981.

[Pa] K. PAWEL: Über sphärische Immersionen in Pseudo-Riemannsche Mannigfaltigkeiten, Dissertation, Köln (2001).

[PR1] K. Pawel, H. Reckziegel: Affine submanifolds and the theorem of Cartan-AmbroseHicks, Kodai Math. J. 25 (2002) 341 - 356

[PR2] K. PAWEL, H. RECKZIEGEL: On the existence of sperically bent submanifolds, an analogue of a theorem of E. Cartan, Kodai Math. J. 26 (2003) $199-220$

[R] H. RECKZIEGEL: On the problem whether the image of a given differentiable map into a Riemannian manifold is contained in a submanifold with parallel second fundamental form, J. reine angew. Math. 325 (1981) 87 -103

[S] W. Strübing: Symmetric submanifolds of Riemannian manifolds, Math. Ann. 245 (1979) $37-44$

[Ta] M. TAKeuchi: Parallel submanifolds of space forms, in: Manifolds and Lie Groups, Papers in Honour of Y. Matsushima, 429 - 447, Birkhäuser 1981

[Ts] K. Tsukada: Totally geodesic submanifolds and curvature-invariant subspaces, Kodai Math. J. 19 (1996) $395-437$.

[V1] J. Vilms: Totally geodesic maps, J. of Diff. Geom. 4 (1970) $73-79$.

[V2] J. ViLms: Submanifolds of euclidean space with parallel second fundamental form, Proc. Amer. Math. Soc. 32 (1972) 263 - 267.

[W] B. Wettstein Congruence and existence of differentiable maps. Diss. ETH 6252. Zürich: Eidgenössische Technische Hochschule (1978) 


\section{Zusammenfassung in deutscher Sprache}

In einer riemannschen Mannigfaltigkeit $N$ sind die totalgeodätischen Untermannigfaltigkeiten diejenigen, welche man als ungekrümmt bezeichnen kann. Im Allgemeinen kommen totalgeodätische Untermannigfaltigkeiten einer Dimension $\geq 2$ und $<\operatorname{dim} N$ sehr selten vor. Bezüglich der Existenzfrage hat É. CARTAN folgendes Kriterium bewiesen:

Theorem (É. Cartan). Gegeben seien ein Punkt $p \in N$, ein linearer Unterraum $V \varsubsetneqq T_{p} N$ einer Dimension $\geq 1$ und eine sternförmige Umgebung $U$ von 0 in $V$, so daß die Exponentialabbildung $\exp _{p}^{N}$ von $N$ in $p$ auf $U$ definiert ist und dabei eine injektive Immersion in $N$ ist. Dann ist das "Geodätischen-Schirmchen" $M_{U}(p, V):=\exp _{p}^{N}(U)$ eine totalgeodätische Untermannigfaltigkeit von $N$ genau dann, wenn für jedes $u \in U$ die Parallelverschiebung $V_{u}$ von $V$ längs des geodätischen Bogen $c_{u}:[0,1] \rightarrow N, t \mapsto \exp _{p}^{N}(t u)$,

$$
\left.V_{u}:=\underset{0}{\left(\| c_{u}\right.}\right)^{N}(V) \subset T_{\exp _{p}^{N}(u)} N
$$

krümmungsinvariant ist ("Integrabilitäsbedingung"), das heißt $R\left(v, v^{\prime}\right) v^{\prime \prime} \in V_{u}$ für alle $v, v^{\prime}, v^{\prime \prime} \in V_{u}$. Man beachte: Es ist $p \in M_{U}(p, V)$ und $T_{p}\left(M_{U}(p, V)\right)=V$.

Ein bekannter Spezialfall dieses Theorems ist die Beziehung zwischen Lietripel-Systemen und totalgeodätischen Untermannigfaltigkeiten in der Theorie der symmetrischen Räume. - In dieser Doktorarbeit wird das analoge Problem für Untermannigfaltigkeiten mit paralleler zweiten Fundamentalform, in der Literatur auch parallele Untermannigfaltigkeiten genannt, gelöst; in dieser Arbeit wird die Terminologie II-parallele Untermannigfaltigkeit bevorzugt, welche explizit auf die zweite Fundamentalform hinweist. Eine Übersicht von Resultaten über II-parallele Untermannigfaltigkeiten findet man in [L].

In reellen Raumformen wurden die II-parallelen Untermannigfaltigkeiten durch FERUs, TAKeuchi, BACKes and ReckzIEgel [F, Ta, BR] klassifiziert; inbesondere wurde gezeigt, daß diese stets offene Teilmengen vollständiger II-paralleler Untermannigfaltigkeiten sind, und daß letzere gerade die (extrinsisch) symmetrischen Untermannigfaltigkeiten im Sinne von FERUS $[F]$ sind. In symmetrischen Räumen wurden die symmetrischen Untermannigfaltigkeiten durch Naitoh, Takeuchi, Berndt, Eschenburg, Tsukada und andere klassifiziert (siehe [NT, B-T]), nachdem NAITOH schon in [Na1] gezeigt hatte, daß symmetrische Untermannigfaltigkeiten stets II-parallel sind.

Falls $M \subset N$ eine II-parallele Untermannigfaltigkeit ist, so ist jede Geodätische von $M$ eine Helix im umgebenden Raum. (Unter dem Begriff Helix sind Geodätische, Kreise und Schraubenlinien eingeschlossen.) Daher sollte das Geodätischen-Schirmchen aus CARTANs Theorem für das in dieser Arbeit behandelte Problem durch ein Helix-Schirmchen $M_{U}(p, W, b)$ ersetzt werden (siehe Proposition 8); dabei ist $p$ ein beliebiger Punkt in $N, W \subset T_{p} N$ der vorgeschriebene Tangentialraum für die gesuchte II-parallele Untermannigfaltigkeit $M, b: W \times W \rightarrow W^{\perp}$ ist die vorgeschriebene zweite Fundamentalform für $M$ in $p$ und $U$ eine geeignet kleine sternförmige Umgebung von 0 in $W$. In der Tat realisiert das Helix-Schirmchen stets die Anfangsbedingung $(p, W, b)$; falls $W$ krümmungsinvariant ist, so verschwindet außerdem die kovariante Ableitung der zweiten Fundamentalform von $M_{U}(p, W, b)$ zumindest in $p$ (siehe Proposition 8). 
Um etwas Einblick in das Hauptresultat (Theorem 1) zu bekommen, werde der Raum End $_{-}\left(T_{p} N\right)$ der schiefadjungierten Endomorphismen von $T_{p} N$ eingeführt und sein linearer Unterraum

$$
\mathbb{M}_{W}:=\left\{A \in \operatorname{End}_{-}\left(T_{p} N\right) \mid A(W) \subset W^{\perp} \text { und } A\left(W^{\perp}\right) \subset W\right\} .
$$

Definition 1 assoziiert mit $b$ eine lineare Abbildung $A^{b}: W \rightarrow \mathbb{M}_{W}, u \mapsto A_{u}^{b}$. Als Analogon zu den Geodätischen in CarTans Theorem wird dann für jedes $u \in W$ die Helix $c_{u}$ unter Zuhilfenahme der 1-Parameter-Untergruppe $t \mapsto \operatorname{Exp}\left(t A_{u}^{b}\right) \in \mathrm{SO}\left(T_{p} N\right)$ definiert (siehe Definition 3); diese Konstruktion läßt den "Schraubenlinien-Charakter" der Helices deutlich hervortreten. Das Helix-Schirmchen wid nun durch

$$
M_{U}(p, W, b):=\left\{c_{u}(1) \mid u \in U\right\}
$$

definiert. Es sollte noch erwähnt werden, daß eine II-parallele Untermannigfaltigkeit $M$ zu den Anfangsdaten $(p, W, b)$ im Falle ihrer Existenz stets ein solches Helix-Schirmchen $M_{U}(p, W, b)$ als offene Teilmenge enthält (siehe Proposition 5(c) und Corollary 4).

Lemma 4 zeigt, wie die zweite Fundamentalform einer Untermannigfaltigkeit $M \subset N$ die "Bewegung" der Tangentialräume von $M$ in $T N$ längs einer Kurve $c: J \rightarrow M$ mit $c(0)=p$ bestimmt: Indem man die zweite Fundamentalform von $M$ längs $c$ zurück nach $T_{p} N$ parallel verschiebt (und die oben erwähnte Abbildung $b \mapsto A^{b}$ benutzt) erhält man eine Funktion $A: J \rightarrow$ End $_{-}\left(T_{p} N\right)$, und ist dann $g: J \rightarrow \mathrm{SO}\left(T_{p} N\right)$ die Lösung der Differentialgleichung $g^{\prime}(t)=g(t) \circ A(t)$ mit $g(0)=\operatorname{id}_{T_{p} N}$, so wird $T_{p} M$ (bzw. $\left.\perp_{p} M\right)$ von der die linearen Isometrie

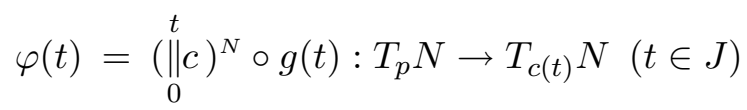

via Paralleltransport in $M$ (bzw. im Normalenbündel $\perp M$ ) auf $T_{c(t)} M$ (bzw. auf $\perp_{c(t)} M$ ) abgebildet. Ist $c_{u}$ eine Geodätische einer II-parallelen Untermannigfaltigkeit mit $\dot{c}_{u}(0)=u$, so stellt sich heraus, daß dieser "an $M$ gespaltene Paralleltransport" von $T_{p} N$ längs $c_{u}$ für jedes $t \in J$ gerade die Isometrie

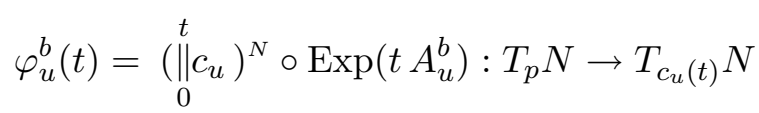

ist (siehe Proposition 5(c)). Daher ist in CARTANs oben erwähntem Theorem der Paralleltransport längs $c_{u}$ durch diese Isometrien $\varphi_{u}^{b}(t)$ zu ersetzen; weiterhin sollte $V_{u}$ durch $\xi_{u}(t):=\varphi_{u}^{b}(t)(W)$ ersetzt werden; und zusätzlich hat man eine bilineare Abbildung $h_{u}(t)$ : $\xi_{u}(t) \times \xi_{u}(t) \rightarrow \xi_{u}(t)^{\perp}$ zu konstruieren, indem man nämlich $b$ via $\varphi_{u}^{b}(t)$ nach $T_{c_{u}(t)} N$ transportiert. Nun besagt Theorem 1:

Das Helix-Schirmchen $M_{U}(p, W, b)$ ist genau dann II-parallel wenn für alle $u \in W$ die Unterräume $\xi_{u}(t)$ krümmungsinvariant und die bilinearen Abbildungen $h_{u}(t)$ semiparallel sind (siehe Definition 1(b)).

In [PR2] haben PAwel und RECKzIEgel schon das analoge Problem für sphärische Untermannigfaltigkeiten (einer Unterklasse der II-parallelen Untermannigfaltigkeiten) gelöst, indem sie ein sogennantes "Kreislinien-Schirmchen" (circular umbrella) konstruiert haben.

Aus Korollar 5 erkennt man, daß in einem Raum konstanter Krümmung genau dann eine II-parallele Untermannigfaltigkeit $\mathrm{zu}$ den Anfangsdaten $(p, W, b)$ existiert, wenn $b R_{p}^{N}$ semiparallel ist. Nun gibt es eine wohlbekannte andere tensorielle Bedingung für die Existenz II-paralleler Untermannigfaltigkeiten in Standardräumen, die von D. Ferus für den Fall 
$N=\mathbb{R}^{n}$ in $[\mathrm{F}]$ entdeckt und später für die anderen Standardräume konstanter Krümmung in [BR] ausgeweitet wurde, daß nämlich eine gewisse bilineare Abbildung $L: W \times W \rightarrow \operatorname{End}(W)$, die mit dem Tripel $(p, W, b)$ assoziiert wird, ein sogenanntes euklidisches Jordan-Tripel-System ist. In Abschnitt 4 werden Ferus Rechnungen auf eine solche Weise präsentiert, daß man die Übereinstimmung seiner und unserer Resultate erkennen kann (siehe Theorem 3).

Zum Beweis von Theorem 1 in Abschnitt 5 wird zur Behandlung der Differentialgeometrie (bis zu zweiter Ordnung) von beliebigen $m$-dimensionalen Untermannigfaltigkeiten $M \subset N$ ein Kalkül entwickelt, der zunächst "frei von Untermannigfaltigkeiten" ist. Da die Spaltung $T_{p} N=T_{p} M \oplus \perp_{p} M$ für das Studium einer Untermannigfaltigkeit $M$ in einem Punkt $p \in M$ entscheidend ist, werden das Graßmannbündel $\tau: \hat{N} \rightarrow N$ (dessen Fasern die Graßmannmannigfaltigkeiten $\hat{N}_{p}:=\mathrm{G}_{m}\left(T_{p} N\right)$ sind) und folgende Bündel über $\hat{N}$ betrachtet: das zurückgezogene Tangentialbündel $\mathbb{E}:=\hat{N} \times{ }_{N} T N \rightarrow \hat{N}$ (dessen Faser über $W \in \hat{N} T_{\tau(W)} N$ ist), die kanonische Spaltung $\mathbb{E}=\mathbb{T} \oplus \Perp \Perp$ gegeben ist, das Hauptfaserbündel $\mathbb{P}$ der angepaßten orthonormalen Basen von $\mathbb{E}$, das Vektorbündel $\mathbb{B}$ der "möglichen" zweiten Fundamentalformen, welches durch $\mathbb{B}_{W}:=\mathrm{L}^{2}\left(W, W^{\perp}\right)$ gegeben wird, und das Vektorbündel $\mathbb{M}$, dessen Fasern $\mathbb{M}_{W}$ schon weiter oben beschrieben wurden. Das Bündel $\mathbb{P}$ ist auf kanonische Weise mit einem linearen Zusammenhang $\mathcal{H}^{\mathbb{P}}$ versehen, welcher eine ausgezeichnete kovariante Ableitung auf den assoziierten Vektorbündeln $\mathbb{E}, \mathbb{B}$ and $\mathbb{M}$ induziert. Insbesondere ist die Parallelverschiebung in $\mathbb{E}$ eine "universelle gespaltene Parallelverschiebung", sie beschreibt also die "an $M$ gespaltene Parallelverschiebung" für beliebige $m$-dimensionale Untermannigfaltigkeiten $M$. Wird weiterhin die zweite Fundamentalform einer $m$-dimensionalen Untermannigfaltigkeit $M$ als Schnitt in $\mathbb{B}$ längs der Gaußabbildung betrachtet, so stimmt ihre kovariante Ableitung (die gerade erwähnte) mit derjenigen überein, die gewöhnlicherweise in der Untermannigfaltigsgeometrie verwendet wird. Das Schlüsselobjekt in dieser Arbeit ist eine gewisse 1-Differentialform $\hat{\nu}$ auf $\hat{N}$ mit Werten im Bündel $\mathbb{M}$, die die zweite Fundamentalform beliebiger $m$-dimensionaler Untermannigfaltigkeiten von $N$ "enthält"; mit ihrer Hilfe lassen sich universelle Gauß- und Weingartengleichungen formulieren und eine universelle Formel, die die Krümmungsgleichungen von Gauß, Codazzi and Ricci zusammenfaßt (siehe (75), (78) und Example 9).

Andererseits ist für jedes $q \in N$ und $W \in \hat{N}_{q}$ die Einschränkung $\hat{\nu} \mid T_{W} \hat{N}_{q}$ der wohlbekannte Isomorphismus zwischen dem Tangentialraum des symmetrischen Raumes $\mathrm{G}_{m}\left(T_{q} N\right)$ im "Punkt" $W$ und dem Unterraum $\mathbb{M}_{W}$ der Liealgebra End $\left(T_{q} N\right)$ der Transformationsgruppe $\mathrm{O}\left(T_{q} N\right)$ (siehe Remark 7 und Theorem 4). Diese Tatsache ermöglicht die Konstruktion eines linearen Zusammenhangs $\hat{\nabla}$ (mit Torsion) auf der Manigfaltigkeit $\hat{N}$ (siehe Abschnitt 6), welcher - für das behandelte Problem - optimal an die Geometrie des Graßmannbündels angepaßt zu sein scheint. In der Tat wird für jede $m$-dimensionale Untermannigfaltigkeit $M$ eine Beziehung zwischen der kovarianten Ableitung ihrer zweiten Fundamentalform und der zweiten Fundamentalform der Gaußabbildung $\xi^{M}$ von $M$ (siehe Theorem 6) hergeleitet; als ein Korollar dieses Theorems erhält man die folgende geometrische Einsicht:

$M$ ist genau dann II-parallel, wenn $\xi^{M}$ eine affine Abbildung in $(\hat{N}, \hat{\nabla})$ ist.

Das eben erwähnte Theorem und das Korollar verallgemeinern VILMS wohlbekannte Resultate aus [V2] bezüglich Untermannigfaltigkeiten im euklidischen Raum.

Weitere Objekte, die in dieser Arbeit eine entscheidende Rolle spielen, sind die mit den bilinearen Abbildungen $b \in \mathbb{B}_{W}$ assoziierten $m$-dimensionale Unterräume $\mathcal{T}_{b} \subset T_{W} \hat{N}$ (siehe 
Abschnitt 7). Für $m$-dimensionale Untermannigfaltigkeiten spielen diese ausgezeichneten Unterräume eine ähnliche Rolle wie die Beschleunigungsvektoren $\in T(T N)$ für Kurven in $N$, die ebenfalls nicht das gesammte Tangentialbündel zweiter Ordnung von $N$ ausfüllen (siehe Theorem 7). Solche "Tangentialräume höherer Ordnung" von Untermannigfaltigkeiten wurden bereits von AmBrose in [A] untersucht; der Vergleich seiner Untersuchung und meiner Ergebnisse wird in Theorem 7 und Proposition 16 dargestellt. Als Nebenprodukt erhalte ich ein neues Kriterium für dir Involutivität einer Distribution (siehe Corollary 11).

Nun läßt sich schließen: Die Konstruktion einer Untermannigfaltigkeit $M \subset N$ mit paralleler zweiter Fundamentalform zu vorgegebenen Anfangsbedingungen $(p, W, b)$ ist äquivalent zur Konstruktion, einer affinen (=autoparallelen) Untermannigfaltigkeit $\hat{M}$ in $(\hat{N}, \hat{\nabla})$ zu den Anfangsbedingungen $\left(W, \mathcal{T}_{b}\right)$. Als einziger Kandidat für $\hat{M}$ kommt nur das GeodätischenSchirmchen aus CARTANs Theorem in Frage. Wie in [PR1] gezeigt wurde bleibt dieses Theorem in jeder "affinen" Mangfaltigkeit $(\hat{N}, \hat{\nabla})$ gültig, solange man nur die Bedingung " $V_{u}$ ist krümmungsinvariant" durch " $V_{u}$ ist krümmungs- und torsionsinvariant" ersetzt. In Proposition 14 werden diese "Integrabilitätsbedingungen" für die Affinität des GeodätischenSchirmchens in $\hat{N}$ in Integrabilitätsbedingungen für die Parallelität der zweiten Fundamentalform des Helix-Schirmchens $M_{U}(p, W, b)$ übersetzt. Danach ist der Beweis des Hauptresultats schnell erbracht (siehe Abschnitt 8).

Ist der Inhalt von Theorem 1 rein lokaler Natur, so wird in Abschnitt 9 eine globale Version dieses Theorems bewiesen. Ist insbesondere $N$ ein symmetrischer Raum, so existiert zu vorgeschriebenen Anfangsdaten $(p, W, b)$ schon eine II-parallele isometrische Immersion von einer vollständigen riemannschen Mannigfaltigkeit in $N$, falls nur ein beliebig kleines HelixSchirmchen $M_{U}(p, W, b)$ II-parallel ist. Diese lokale Bedingung kann an Hand von endlich vielen tensoriellen Gleichungen getestet werden (siehe Theorem 2).

Der Einfachheit halber habe ich in der Regel in dieser Arbeit angenommen, daß die riemannsche Metrik von $N$ positiv definit ist. Im Falle einer pseudoriemannschen Mannigfaltigkeit $N$ vom Index $k \in\{1, \ldots, \operatorname{dim} N-1\}$ darf der vorgeschriebene Tangentialraum $W \in \mathrm{G}_{m}\left(T_{p} N\right)$ in Theorem 1 nicht (bezüglich der Metrik von $N$ ) ausgeartet sein; falls dieser pseudoeuklidische Vektorraum den Index $\ell \in\{0, \ldots, \min \{m, k\}\}$ hat, so muß das Graßmannbündel $\tau: \hat{N} \rightarrow N$ auf die Menge dieser gleichartigen Unterräume beschränkt werden. In Abschnitt 11 werden diese Tatsache und die notwendigen Abänderungen der Argumentation beschrieben. Hier will ich nur eine Tatsache erwähnen. In einer vollständigen riemannschen Mannigfaltigkeit sind die maximalen Helices auf der ganzen reellen Achse $\mathbb{R}$ definiert. Im pseudoriemannschen Fall ist dies nicht unbedingt wahr, da eine geodätisch vollständige pseudoriemannsche Mannigfaltigkeit im allgemeinen nicht total vollständig ist; in Proposition 3 wird eine wesentliche Ausnahme beschrieben, wenn nämlich $N$ ein pseudoriemannscher symmetrischer Raum (allgemeiner ein reduktiver homogener Raum mit seinem kanonischen Zusammnenhang) ist; obwohl diese Tatsache bekannt ist, gebe ich einen Beweis hierfür, da ich in den Lehrbüchern keinen finden konnte.

Soweit wurde der Inhalt dieser Arbeit in Bezug auf Theorem 1, das Analogon zu CARTANs am Anfang dieser Zusammenfassung zitiertem Theorem, beschrieben. In der Tat besteht das Ziel meiner Arbeit auch darin, tieferen Einblick in die Geometrie II-paralleler Untermannigfaltigkeiten zu geben. Zum Beispiel scheinen die Beschreibung der Helices in Definition 3 und die Bewegung der Tangentialräume einer II-parallelen Untermannigfaltigkeit längs einer Geodätischen in Proposition 5 (diese Ergebnisse wurden schon in [JR] präsentiert) neu zu sein. Tatschlich kann ich dieses Verhalten der Tangentialräume in einer etwas allgemeineren Situation 
beweisen (siehe Proposition 6 und Corollary 3). Weiterhin zeigt Corollary 4, daß die wohlbekannte Starrheit II-paralleler Untermannigfaltigkeiten auf solche Untermannigfaltigkeiten zutrifft, in denen nur eine $k$-te kovariante Ableitung der zweiten Fundamentalform identisch verschwindet. Corollary 2 sollte ebenfalls erwähnt werden; es besagt daß eine II-parallele Untermannigfaltigkeit eines lokalsymmetrischen Raumes wieder lokalsymmetrisch ist, ein Resultat das in [Na1] ohne Beweis erwähnt wird. Da die zweite Fundamentalform $h^{M}$ einer Untermannigfaltigkeit $M$ das primäre Objekt dieser Arbeit ist, möchte ich auf Proposition 1 hinweisen, in der ich eine Interpretation von $h^{M}$ über das infinitesimale Kippen der Tangentialräume von $M$ längs Kurven gebe. In Abschnitt 6 wird gezeigt, daß dieser Sachverhalt auch unter Zuhilfenahme der "universellen zweiten Fundamentalform " $\hat{\nu}$ aus einer sehr allgemeinen Beobachtung (assoziierte Faserbündel betreffend) folgt (siehe Lemma 6).

Genauso gut hätte ich die Verallgemeinerung von VILMs Resultat (Theorem 6 und Corollary 10) als zentrales Objekt meiner Dissertation bezeichnen können. Daher ist es interessant, den linearen Zusammenhang $\hat{\nabla}$ mit dem Levi-Civita Zusammenhang $\nabla^{L C}$ der kanonischen riemannschen Metrik des Graßmannbündels zu vergleichen; in Proposition 12 werden die Haupteigenschaften von $\nabla^{L C}$ beschrieben; in Kapitel 6 (Theorem 5, Corollary 8 und 9) findet man den Vergleich der beiden Zusammenhänge.

Der letzte Abschnitt behandelt den Fall, daß die riemannsche Mannigfaltigkeit $N$ mit einer zusätzlichen "parallelen" geometrischen Struktur versehen ist (siehe Definition 13). Dann kann man die Frage stellen, wann II-parallele Untermannigfaltigkeiten mit dieser Struktur verträglich sind. Startet man mit einem ausgezeichneten Unterraum $W_{0} \in \hat{N}$, so läßt sich ein paralleles Unterbündel $\hat{N}\left(W_{0}\right) \rightarrow N$ von $\tau: \hat{N} \rightarrow N$ konstruieren, dessen Fasern symmetrische Unterräume der Fasern von $\tau$ sind und dessen Bündelraum dann auch eine affine Untermannigfaltigkeit von $(\hat{N}, \hat{\nabla})$ ist (siehe Punkt (i) von Example 16(c) und Proposition 24). Eine $m$-dimensionale II-parallele isometrische Immersion $f: M \rightarrow N$ wird als kompatibel mit der Zusatzstruktur auf $N$ angesehen, wenn die Gaußabbildung $\xi^{f}$ die Bedingung $\xi^{f}(M) \subset \hat{N}\left(W_{0}\right)$ erfüllt; und dies ist schon dann der Fall, wenn nur der 1-Jet von $\xi^{f}$ in einem Punkt $p \in M$ die entsprechende Bedingung erfüllt (siehe Theorem 12). Daß diese abstrakte Theorie wirklich Sinn macht, wird an zwei Beispielen verdeutlich, nämlich an komplexen und Lagrangen II-parallelen Untermannigfaltigkeiten in Kählermannigfaltigkeiten (siehe Corollary 14) und an extrinsch symmetrischen Untermannigfaltigkeiten in symmetrischen Räumen (siehe Corollary 15 und Theorem 13).

Zuletzt sind noch einige Worte zu Abschnitt 10 angebracht. Darin wird die Theorie der IIparallelen Untermannigfaltigkeiten auf Streifen längs beliebiger differenzierbarer Abbildungen $f: M \rightarrow N$ verallgemeinert. (Streifen sind als natürliche Verallgemeinerung von Immersionen anzusehen, da doch die Gaußabbildung einer Immersion ein Streifen ist.) Aufgrund der in dieser Dissertation entwickelten Theorie kann man damit sehr einfach das Hauptergebnis der Arbeit $[R]$ beweisen; dies ist ein Kriterium dafür, wann eine differenzierbare Abbildung in eine gegebene II-parallele Untermannigfaltigkeit hinein abbildet (Theorem 11); dieses Resultat enthält insbesondere ERBACHERs Theorem über die Reduktion der Kodimension für isometrische Immersionen in reellen Raumformen (siehe $[\mathrm{E}]$ ). 


\section{Versicherung}

Ich versichere, daß ich die von mir vorgelegte Dissertation selbständig angefertigt, die benutzten Quellen und Hilfsmittel vollständig angegeben und die Stellen der Arbeit - einschließlich Tabellen, Karten und Abbildungen - die anderen Werken im Wortlaut oder dem Sinn nach entnommen sind, in jedem Einzelfall als Entlehnung kenntlich gemacht habe; daß diese Dissertation noch keiner anderen Fakultät oder Universität zur Prüfung vorgelegen hat; daß sie-abgesehen von unten angegebenen Teilpublikationen- noch nicht veröffentlicht worden ist sowie, daß ich eine solche Veröffentlichung vor Abschluß des Promotionsverfahrens nicht vornehmen werde.

Die Bestimmungen der Promotionsordnung sind mir bekannt. Die von mir vorgelegte Dissertation ist von Professor Dr. H. Reckziegel betreut worden.

Teilpublikation: T. Jentsch, H. Reckziegel: Submanifolds with parallel second fundamental form studied via the Gauß map, Annals of Global Analysis and Geometry 29 (2006) 51 - 93.

Köln, den 3. Mai 2005 



\section{Lebenslauf}

Name

geboren am

Staatsangehörigkeit

Familienstand

Eltern

Geschwister

Grundschule 1981 - 1985

Gymnasium 1985 - 1994

Abitur 17.05.1994

Zivildienst 1994 - 1995

Studium 1995 - 2002

Diplom 29.11.2002

Doktorand 2002 - 2005
Tillmann Jentsch

23.03.1975

deutsch

ledig

Siegfried Jentsch und Ute Jentsch, geborene Schmidt

Boris Peter Jentsch

Gemeinschaftsgrundschule Kippekausen in Bergisch Gladbach Otto Hahn Gymnasium in Bergisch Gladbach am Otto Hahn Gymnasium in der Werkstatt für Behinderte in Refrath am mathematischen Institut der Universität zu Köln Erlangung des Diploms in Mathematik am mathematischen Institut der Universität zu Köln 